\title{
Archiver Effects on the Performance of State-of-the-art Multi- and Many-objective Evolutionary Algorithms
}

\author{
Leonardo C. T. Bezerra \\ Instituto Metrópole Digital, \\ Universidade Federal do Rio Grande \\ do Norte \\ Natal, RN, Brazil \\ leobezerra@imd.ufrn.br
}

\author{
Manuel López-Ibáñez \\ Alliance Business School \\ University of Manchester \\ Manchester, UK \\ manuel.lopez-ibanez@manchester.ac.
}

\author{
Thomas Stützle \\ IRIDIA, CoDE \\ Université Libre de Bruxelles \\ Brussels, Belgium \\ stuetzle@ulb.ac.be
}

\section{ACM Reference Format:}

Leonardo C. T. Bezerra, Manuel López-Ibáñez, and Thomas Stützle. 2019. Archiver Effects on the Performance of State-of-the-art Multi- and Manyobjective Evolutionary Algorithms: Supplementary Material. In Proceed ings of the Genetic and Evolutionary Computation Conference 2019 (GECCO '19). ACM, New York, NY, USA, Article 4, 98 pages. https://doi.org/10.1145/ 3321707.3321789

This supplementary material provides all the data required for the analysis of the main paper that was omitted for brevity. For better navigation, we provide links to the contents of this supplementary material.

Tables 1-2 list the numerical parameters selected by irace specific to CMA and MOEA/D, respectively. General parameter settings used by most MOEAs are given in Tables 3 and 4 .

Figures 1-4 respectively show boxplots of the performance differences between runs from MOEAs with and without unbounded archiving on selected problems with $n_{\mathrm{var}}=40$, $F E_{\text {max }}=2500$ and $M \in\{2,3,5,10\}$. Each of these plots depicts performance differences according to the $I_{H}^{r d}, I_{\epsilon+}$ and $I_{I G D}$ (from top to bottom). The same pattern is repeated for $F E_{\max }=10000$ (Figures 5-8) and $F E_{\max }=40000$ (Figures 912).

Figures 13-16 respectively show boxplots of the performance differences between runs from MOEAs with and without bounded archiving, grouped by benchmark function, on scenarios with $F E_{\max }=40000$ and $M \in\{2,3,5,10\}$. Each of these plots depicts performance differences according to the $I_{H}^{r d}, I_{\epsilon+}$ and $I_{I G D}$ (from top to bottom).

Figures 17-20 respectively show boxplots of the performance differences according to the $I_{H}^{r d}$ between runs from MOEAs with and without unbounded archiving on all problems with $F E_{\text {max }}=2500$ and $M \in\{2,3,5,10\}$. The same pattern is repeated for $F E_{\max }=10000$ (Figures 21-24) and $F E_{\max }=40000$ (Figures 25-28). In addition, this more general pattern is also

Permission to make digital or hard copies of all or part of this work for personal or classroom use is granted without fee provided that copies are not made or distributed for profit or commercial advantage and that copies bear this notice and the full citation on the first page. Copyrights for components of this work owned by others than ACM must be honored. Abstracting with credit is permitted. To copy otherwise, or republish, to post on servers or to redistribute to lists, requires prior specific permission and/or a fee. Request permissions from permissions@acm.org.

GECCO '19, July 13-17, 2019, Prague, Czech Republic

(C) 2019 Association for Computing Machinery.

ACM ISBN 978-1-4503-6111-8/19/07 ..\$15.00

https://doi.org/10.1145/3321707.3321789 used to depict results for $I_{\epsilon+}$ (Figures 29-40) and $I_{I G D}$ (Figures 41-52).

Figures 53-56 respectively show boxplots of the performance according to the $I_{H}^{r d}$ of MOEA runs with unbounded archiving on all problems with $F E_{\max }=2500$ and $M \in\{2,3,5,10\}$. The same pattern is repeated for $F E_{\max }=10000$ (Figures 57-60) and $F E_{\max }=40000$ (Figures 61-64). In addition, this more general pattern is also used to depict results for $I_{\epsilon+}$ (Figures 65-76), $I_{I G D}$ (Figures 77-88), and $I_{I G D+}$ (Figures 89-100).

\section{List OF TABLES}

1 Parameters selected by irace specific to MO-CMA-ES.

2 Parameters selected by irace specific to MOEA/D.

3 Underlying EA parameters selected by irace for MOEAs. IBEA uses $I_{\epsilon+}$ for all scenarios.

4 Underlying EA parameters selected by irace for MOEAs. SPEA2 uses $t_{\text {size }}=8$ when $M<10, t_{\text {size }}=2$ on $\langle 10,10\rangle$, and $t_{\text {size }}=4$ on $\langle 10,40\rangle$. NSGA-III uses $H_{2}=2$ on all $M=10$ scenarios but $\langle 10,40\rangle$, where $H_{2}=1$.

\section{List OF FigURES}

1 Performance differences between runs from MOEAs with and without unbounded archiving on the scenario with $M=2$ and $F E_{\max }=2500$ for selected problems with $n_{\mathrm{var}}=40$. From top to bottom, $I_{H}^{r d}, I_{\epsilon+}$, and $I_{I G D}$.

2 Performance differences between runs from MOEAs with and without unbounded archiving on the scenario with $M=3$ and $F E_{\max }=2500$ for selected problems with $n_{\mathrm{var}}=40$. From top to bottom, $I_{H}^{r d}, I_{\epsilon+}$, and $I_{I G D}$.

3 Performance differences between runs from MOEAs with and without unbounded archiving on the scenario with $M=5$ and $F E_{\max }=2500$ for selected problems with $n_{\mathrm{var}}=40$. From top to bottom, $I_{H}^{r d}, I_{\epsilon+}$, and $I_{I G D}$.

4 Performance differences between runs from MOEAs with and without unbounded archiving on the scenario with $M=10$ and $F E_{\max }=2500$ for selected problems with $n_{\mathrm{var}}=40$. From top to bottom, $I_{H}^{r d}, I_{\epsilon+}$, and $I_{I G D}$.
6 
5 Performance differences between runs from MOEAs with and without unbounded archiving on the scenario with $M=2$ and $F E_{\max }=10000$ for selected problems with $n_{\mathrm{var}}=40$. From top to bottom, $I_{H}^{r d}, I_{\epsilon+}$, and $I_{I G D}$.

6 Performance differences between runs from MOEAs with and without unbounded archiving on the scenario with $M=3$ and $F E_{\max }=10000$ for selected problems with $n_{\mathrm{var}}=40$. From top to bottom, $I_{H}^{r d}, I_{\epsilon+}$, and $I_{I G D}$.

7 Performance differences between runs from MOEAs with and without unbounded archiving on the scenario with $M=5$ and $F E_{\max }=10000$ for selected problems with $n_{\mathrm{var}}=40$. From top to bottom, $I_{H}^{r d}, I_{\epsilon+}$, and $I_{I G D}$.

8 Performance differences between runs from MOEAs with and without unbounded archiving on the scenario with $M=10$ and $F E_{\max }=10000$ for selected problems with $n_{\mathrm{var}}=40$. From top to bottom, $I_{H}^{r d}, I_{\epsilon+}$, and $I_{I G D}$.

9 Performance differences between runs from MOEAs with and without unbounded archiving on the scenario with $M=2$ and $F E_{\max }=40000$ for selected problems with $n_{\mathrm{var}}=40$. From top to bottom, $I_{H}^{r d}, I_{\epsilon+}$, and $I_{I G D}$.

10 Performance differences between runs from MOEAs with and without unbounded archiving on the scenario with $M=3$ and $F E_{\max }=40000$ for selected problems with $n_{\mathrm{var}}=40$. From top to bottom, $I_{H}^{r d}, I_{\epsilon+}$, and $I_{I G D}$.

11 Performance differences between runs from MOEAs with and without unbounded archiving on the scenario with $M=5$ and $F E_{\max }=40000$ for selected problems with $n_{\mathrm{var}}=40$. From top to bottom, $I_{H}^{r d}, I_{\epsilon+}$, and $I_{I G D}$.

12 Performance differences between runs from MOEAs with and without unbounded archiving on the scenario with $M=10$ and $F E_{\max }=40000$ for selected problems with $n_{\mathrm{var}}=40$. From top to bottom, $I_{H}^{r d}, I_{\epsilon+}$, and $I_{I G D}$.

13 Boxplots of performance differences for $M=2$ and $F E_{\text {max }}=40000$, grouped by MOEA and benchmark function, depicting the different archiving alternatives. From top to bottom: $I_{H}^{r d}, I_{\epsilon+}$, and $I_{I G D}$.

14 Boxplots of performance differences for $M=3$ and $F E_{\text {max }}=40000$, grouped by MOEA and benchmark function, depicting the different archiving alternatives. From top to bottom: $I_{H}^{r d}, I_{\epsilon+}$, and $I_{I G D}$.

15 Boxplots of performance differences for $M=5$ and $F E_{\text {max }}=40000$, grouped by MOEA and benchmark function, depicting the different archiving alternatives. From top to bottom: $I_{H}^{r d}, I_{\epsilon+}$, and $I_{I G D}$.

16 Boxplots of performance differences for $M=10$ and $F E_{\text {max }}=40000$, grouped by MOEA and benchmark function, depicting the different archiving alternatives. From top to bottom: $I_{H}^{r d}, I_{\epsilon+}$, and $I_{I G D}$.

17 Performance differences according to $I_{H}^{r d}$ between runs from MOEAs with and without unbounded archiving on the scenario with $M=2$ and $F E_{\max }=2500$ for all problems with $n_{\mathrm{var}} \in\{30,40,50\}$.
18 Performance differences according to $I_{H}^{r d}$ between runs from MOEAs with and without unbounded archiving on the scenario with $M=3$ and $F E_{\max }=2500$ for all problems with $n_{\mathrm{var}} \in\{30,40,50\}$.

19 Performance differences according to $I_{H}^{r d}$ between runs from MOEAs with and without unbounded archiving on the scenario with $M=5$ and $F E_{\max }=2500$ for all problems with $n_{\mathrm{var}} \in\{30,40,50\}$.

20 Performance differences according to $I_{H}^{r d}$ between runs from MOEAs with and without unbounded archiving on the scenario with $M=10$ and $F E_{\max }=2500$ for all problems with $n_{\mathrm{var}} \in\{30,40,50\}$.

21 Performance differences according to $I_{H}^{r d}$ between runs from MOEAs with and without unbounded archiving on the scenario with $M=2$ and $F E_{\max }=10000$ for all problems with $n_{\mathrm{var}} \in\{30,40,50\}$.

22 Performance differences according to $I_{H}^{r d}$ between runs from MOEAs with and without unbounded archiving on the scenario with $M=3$ and $F E_{\max }=10000$ for all problems with $n_{\mathrm{var}} \in\{30,40,50\}$.

23 Performance differences according to $I_{H}^{r d}$ between runs from MOEAs with and without unbounded archiving on the scenario with $M=5$ and $F E_{\max }=10000$ for all problems with $n_{\mathrm{var}} \in\{30,40,50\}$.

24 Performance differences according to $I_{H}^{r d}$ between runs from MOEAs with and without unbounded archiving on the scenario with $M=10$ and $F E_{\max }=10000$ for all problems with $n_{\mathrm{var}} \in\{30,40,50\}$.

25 Performance differences according to $I_{H}^{r d}$ between runs from MOEAs with and without unbounded archiving on the scenario with $M=2$ and $F E_{\max }=40000$ for all problems with $n_{\mathrm{var}} \in\{30,40,50\}$.

26 Performance differences according to $I_{H}^{r d}$ between runs from MOEAs with and without unbounded archiving on the scenario with $M=3$ and $F E_{\max }=40000$ for all problems with $n_{\mathrm{var}} \in\{30,40,50\}$.

27 Performance differences according to $I_{H}^{r d}$ between runs from MOEAs with and without unbounded archiving on the scenario with $M=5$ and $F E_{\max }=40000$ for all problems with $n_{\mathrm{var}} \in\{30,40,50\}$.

28 Performance differences according to $I_{H}^{r d}$ between runs from MOEAs with and without unbounded archiving on the scenario with $M=10$ and $F E_{\max }=40000$ for all problems with $n_{\mathrm{var}} \in\{30,40,50\}$.

29 Performance differences according to $I_{\epsilon+}$ between runs from MOEAs with and without unbounded archiving on the scenario with $M=2$ and $F E_{\max }=2500$ for all problems with $n_{\mathrm{var}} \in\{30,40,50\}$.

30 Performance differences according to $I_{\epsilon+}$ between runs from MOEAs with and without unbounded archiving on the scenario with $M=3$ and $F E_{\max }=2500$ for all problems with $n_{\mathrm{var}} \in\{30,40,50\}$. 
31 Performance differences according to $I_{\epsilon+}$ between runs from MOEAs with and without unbounded archiving on the scenario with $M=5$ and $F E_{\max }=2500$ for all problems with $n_{\mathrm{var}} \in\{30,40,50\}$.

32 Performance differences according to $I_{\epsilon+}$ between runs from MOEAs with and without unbounded archiving on the scenario with $M=10$ and $F E_{\max }=2500$ for all problems with $n_{\mathrm{var}} \in\{30,40,50\}$.

33 Performance differences according to $I_{\epsilon+}$ between runs from MOEAs with and without unbounded archiving on the scenario with $M=2$ and $F E_{\max }=10000$ for all problems with $n_{\mathrm{var}} \in\{30,40,50\}$.

34 Performance differences according to $I_{\epsilon+}$ between runs from MOEAs with and without unbounded archiving on the scenario with $M=3$ and $F E_{\max }=10000$ for all problems with $n_{\mathrm{var}} \in\{30,40,50\}$.

35 Performance differences according to $I_{\epsilon+}$ between runs from MOEAs with and without unbounded archiving on the scenario with $M=5$ and $F E_{\max }=10000$ for all problems with $n_{\mathrm{var}} \in\{30,40,50\}$.

36 Performance differences according to $I_{\epsilon+}$ between runs from MOEAs with and without unbounded archiving on the scenario with $M=10$ and $F E_{\max }=10000$ for all problems with $n_{\mathrm{var}} \in\{30,40,50\}$.

37 Performance differences according to $I_{\epsilon+}$ between runs from MOEAs with and without unbounded archiving on the scenario with $M=2$ and $F E_{\max }=40000$ for all problems with $n_{\mathrm{var}} \in\{30,40,50\}$.

38 Performance differences according to $I_{\epsilon+}$ between runs from MOEAs with and without unbounded archiving on the scenario with $M=3$ and $F E_{\max }=40000$ for all problems with $n_{\mathrm{var}} \in\{30,40,50\}$.

39 Performance differences according to $I_{\epsilon+}$ between runs from MOEAs with and without unbounded archiving on the scenario with $M=5$ and $F E_{\max }=40000$ for all problems with $n_{\mathrm{var}} \in\{30,40,50\}$.

40 Performance differences according to $I_{\epsilon+}$ between runs from MOEAs with and without unbounded archiving on the scenario with $M=10$ and $F E_{\max }=40000$ for all problems with $n_{\mathrm{var}} \in\{30,40,50\}$.

41 Performance differences according to $I_{I G D}$ between runs from MOEAs with and without unbounded archiving on the scenario with $M=2$ and $F E_{\max }=2500$ for all problems with $n_{\mathrm{var}} \in\{30,40,50\}$.

42 Performance differences according to $I_{I G D}$ between runs from MOEAs with and without unbounded archiving on the scenario with $M=3$ and $F E_{\max }=2500$ for all problems with $n_{\mathrm{var}} \in\{30,40,50\}$.

43 Performance differences according to $I_{I G D}$ between runs from MOEAs with and without unbounded archiving on the scenario with $M=5$ and $F E_{\max }=2500$ for all problems with $n_{\mathrm{var}} \in\{30,40,50\}$.
44 Performance differences according to $I_{I G D}$ between runs from MOEAs with and without unbounded archiving on the scenario with $M=10$ and $F E_{\max }=2500$ for all problems with $n_{\mathrm{var}} \in\{30,40,50\}$.

45 Performance differences according to $I_{I G D}$ between runs from MOEAs with and without unbounded archiving on the scenario with $M=2$ and $F E_{\max }=10000$ for all problems with $n_{\mathrm{var}} \in\{30,40,50\}$.

46 Performance differences according to $I_{I G D}$ between runs from MOEAs with and without unbounded archiving on the scenario with $M=3$ and $F E_{\max }=10000$ for all problems with $n_{\mathrm{var}} \in\{30,40,50\}$.

47 Performance differences according to $I_{I G D}$ between runs from MOEAs with and without unbounded archiving on the scenario with $M=5$ and $F E_{\max }=10000$ for all problems with $n_{\mathrm{var}} \in\{30,40,50\}$.

48 Performance differences according to $I_{I G D}$ between runs from MOEAs with and without unbounded archiving on the scenario with $M=10$ and $F E_{\max }=10000$ for all problems with $n_{\mathrm{var}} \in\{30,40,50\}$.

49 Performance differences according to $I_{I G D}$ between runs from MOEAs with and without unbounded archiving on the scenario with $M=2$ and $F E_{\max }=40000$ for all problems with $n_{\mathrm{var}} \in\{30,40,50\}$.

50 Performance differences according to $I_{I G D}$ between runs from MOEAs with and without unbounded archiving on the scenario with $M=3$ and $F E_{\max }=40000$ for all problems with $n_{\mathrm{var}} \in\{30,40,50\}$.

51 Performance differences according to $I_{I G D}$ between runs from MOEAs with and without unbounded archiving on the scenario with $M=5$ and $F E_{\max }=40000$ for all problems with $n_{\mathrm{var}} \in\{30,40,50\}$.

52 Performance differences according to $I_{I G D}$ between runs from MOEAs with and without unbounded archiving on the scenario with $M=10$ and $F E_{\max }=40000$ for all problems with $n_{\text {var }} \in\{30,40,50\}$.

53 Boxplots of the $I_{H}^{r d}$ for $M=2$ problems, $n_{\mathrm{var}} \in\{30,40,50\}$ and $F E_{\max }=2500$.

54 Boxplots of the $I_{H}^{r d}$ for $M=3$ problems, $n_{\mathrm{var}} \in\{30,40,50\}$ and $F E_{\max }=2500$.

55 Boxplots of the $I_{H}^{r d}$ for $M=5$ problems, $n_{\mathrm{var}} \in\{30,40,50\}$ and $F E_{\max }=2500$.

56 Boxplots of the $I_{H}^{r d}$ for $M=10$ problems, $n_{\mathrm{var}} \in\{30,40,50\}$ and $F E_{\max }=2500$.

57 Boxplots of the $I_{H}^{r d}$ for $M=2$ problems, $n_{\mathrm{var}} \in\{30,40,50\}$ and $F E_{\max }=10000$.

58 Boxplots of the $I_{H}^{r d}$ for $M=3$ problems, $n_{\mathrm{var}} \in\{30,40,50\}$ and $F E_{\max }=10000$.

59 Boxplots of the $I_{H}^{r d}$ for $M=5$ problems, $n_{\mathrm{var}} \in\{30,40,50\}$ and $F E_{\max }=10000$.

60 Boxplots of the $I_{H}^{r d}$ for $M=10$ problems, $n_{\mathrm{var}} \in\{30,40,50\}$ and $F E_{\max }=10000$. 
61 Boxplots of the $I_{H}^{r d}$ for $M=2$ problems, $n_{\mathrm{var}} \in\{30,40,50\}$ and $F E_{\max }=40000$.

62 Boxplots of the $I_{H}^{r d}$ for $M=3$ problems, $n_{\mathrm{var}} \in\{30,40,50\}$ and $F E_{\max }=40000$.

63 Boxplots of the $I_{H}^{r d}$ for $M=5$ problems, $n_{\mathrm{var}} \in\{30,40,50\}$ and $F E_{\max }=40000$.

64 Boxplots of the $I_{H}^{r d}$ for $M=10$ problems, $n_{\mathrm{var}} \in\{30,40,50\}$ and $F E_{\max }=40000$

65 Boxplots of the $I_{\epsilon+}$ for $M=2$ problems, $n_{\mathrm{var}} \in\{30,40,50\}$ and $F E_{\max }=2500$.

66 Boxplots of the $I_{\epsilon+}$ for $M=3$ problems, $n_{\mathrm{var}} \in\{30,40,50\}$ and $F E_{\max }=2500$.

67 Boxplots of the $I_{\epsilon+}$ for $M=5$ problems, $n_{\mathrm{var}} \in\{30,40,50\}$ and $F E_{\max }=2500$.

68 Boxplots of the $I_{\epsilon+}$ for $M=10$ problems, $n_{\mathrm{var}} \in\{30,40,50\}$ and $F E_{\max }=2500$.

69 Boxplots of the $I_{\epsilon+}$ for $M=2$ problems, $n_{\mathrm{var}} \in\{30,40,50\}$ and $F E_{\max }=10000$.

70 Boxplots of the $I_{\epsilon+}$ for $M=3$ problems, $n_{\mathrm{var}} \in\{30,40,50\}$ and $F E_{\max }=10000$.

71 Boxplots of the $I_{\epsilon+}$ for $M=5$ problems, $n_{\mathrm{var}} \in\{30,40,50\}$ and $F E_{\max }=10000$.

72 Boxplots of the $I_{\epsilon+}$ for $M=10$ problems, $n_{\mathrm{var}} \in\{30,40,50\}$ and $F E_{\max }=10000$.

73 Boxplots of the $I_{\epsilon+}$ for $M=2$ problems, $n_{\mathrm{var}} \in\{30,40,50\}$ and $F E_{\max }=40000$

74 Boxplots of the $I_{\epsilon+}$ for $M=3$ problems, $n_{\mathrm{var}} \in\{30,40,50\}$ and $F E_{\max }=40000$.

75 Boxplots of the $I_{\epsilon+}$ for $M=5$ problems, $n_{\mathrm{var}} \in\{30,40,50\}$ and $F E_{\max }=40000$.

Boxplots of the $I_{\epsilon+}$ for $M=10$ problems, $n_{\mathrm{var}} \in\{30,40,50\}$ and $F E_{\max }=40000$. and $F E_{\max }=2500$. and $F E_{\max }=2500$. and $F E_{\max }=2500$. $\{30,40,50\}$ and $F E_{\max }=2500$.

81 Boxplots of the $I_{I G D}$ for $M=2$ problems, $n_{\mathrm{var}} \in\{30,40,50\}$ and $F E_{\max }=10000$

82 Boxplots of the $I_{I G D}$ for $M=3$ problems, $n_{\mathrm{var}} \in\{30,40,50\}$ and $F E_{\max }=10000$.

83 Boxplots of the $I_{I G D}$ for $M=5$ problems, $n_{\mathrm{var}} \in\{30,40,50\}$ and $F E_{\max }=10000$.

84 Boxplots of the $I_{I G D}$ for $M=10$ problems, $n_{\mathrm{var}} \in$ $\{30,40,50\}$ and $F E_{\max }=10000$.

85 Boxplots of the $I_{I G D}$ for $M=2$ problems, $n_{\mathrm{var}} \in\{30,40,50\}$ and $F E_{\max }=40000$.
86 Boxplots of the $I_{I G D}$ for $M=3$ problems, $n_{\mathrm{var}} \in\{30,40,50\}$ and $F E_{\max }=40000$.

87 Boxplots of the $I_{I G D}$ for $M=5$ problems, $n_{\mathrm{var}} \in\{30,40,50\}$ and $F E_{\max }=40000$.

88 Boxplots of the $I_{I G D}$ for $M=10$ problems, $n_{\mathrm{var}} \in$ $\{30,40,50\}$ and $F E_{\max }=40000$.

89 Boxplots of the $I_{I G D+}$ for $M=2$ problems, $n_{\mathrm{var}} \in$ $\{30,40,50\}$ and $F E_{\max }=2500$.

90 Boxplots of the $I_{I G D+}$ for $M=3$ problems, $n_{\mathrm{var}} \in$ $\{30,40,50\}$ and $F E_{\max }=2500$.

91 Boxplots of the $I_{I G D+}$ for $M=5$ problems, $n_{\mathrm{var}} \in$ $\{30,40,50\}$ and $F E_{\max }=2500$.

92 Boxplots of the $I_{I G D+}$ for $M=10$ problems, $n_{\mathrm{var}} \in$ $\{30,40,50\}$ and $F E_{\max }=2500$.

93 Boxplots of the $I_{I G D+}$ for $M=2$ problems, $n_{\mathrm{var}} \in$ $\{30,40,50\}$ and $F E_{\max }=10000$.

94 Boxplots of the $I_{I G D+}$ for $M=3$ problems, $n_{\mathrm{var}} \in$ $\{30,40,50\}$ and $F E_{\max }=10000$.

95 Boxplots of the $I_{I G D+}$ for $M=5$ problems, $n_{\mathrm{var}} \in$ $\{30,40,50\}$ and $F E_{\max }=10000$.

96 Boxplots of the $I_{I G D_{+}}$for $M=10$ problems, $n_{\mathrm{var}} \in$ $\{30,40,50\}$ and $F E_{\max }=10000$.

97 Boxplots of the $I_{I G D+}$ for $M=2$ problems, $n_{\mathrm{var}} \in$ $\{30,40,50\}$ and $F E_{\max }=40000$.

98 Boxplots of the $I_{I G D+}$ for $M=3$ problems, $n_{\mathrm{var}} \in$ $\{30,40,50\}$ and $F E_{\text {max }}=40000$.

99 Boxplots of the $I_{I G D+}$ for $M=5$ problems, $n_{\mathrm{var}} \in$ $\{30,40,50\}$ and $F E_{\text {max }}=40000$.

100 Boxplots of the $I_{I G D+}$ for $M=10$ problems, $n_{\mathrm{var}} \in$ $\{30,40,50\}$ and $F E_{\max }=40000$. 
Table 1: Parameters selected by irace specific to MO-CMA-ES.

\begin{tabular}{|c|c|c|c|c|c|c|c|c|c|c|c|c|c|}
\hline M & $F E_{\max }$ & $\mu$ & Notion of Success & Indicator & Steady-state & $\sigma_{0}$ & $d$ & $p_{\text {target }}$ & $p_{\text {thresh }}$ & $c_{p}$ & $c_{c}$ & $c_{c o v}^{\prime}$ & $u_{c o v}^{\prime}$ \\
\hline \multirow{3}{*}{2} & 2500 & 10 & Individual-based & $I_{H}^{1}$ & $\mathrm{~F}$ & 0.8 & auto & 0.45 & auto & auto & 0.21 & auto & auto \\
\hline & 10000 & 20 & Individual-based & $I_{H}^{1}$ & $\mathrm{~F}$ & 0.09 & 38 & 0.47 & auto & auto & 0.25 & auto & 0.32 \\
\hline & 40000 & 50 & Population-based & $I_{H}^{1}$ & $\mathrm{~F}$ & 0.75 & auto & 0.39 & 0.47 & auto & auto & auto & equal \\
\hline \multirow{3}{*}{3} & 2500 & 10 & Population-based & $I_{H}^{1}$ & $\mathrm{~T}$ & 0.68 & 16 & 0.43 & 0.29 & auto & 0.23 & auto & auto \\
\hline & 10000 & 20 & Population-based & $I_{H}^{1}$ & F & 0.78 & 10 & 0.3 & 0.11 & auto & auto & auto & equal \\
\hline & 40000 & 70 & Population-based & $I_{H}^{1}$ & $\mathrm{~F}$ & 0.78 & auto & 0.43 & 0.26 & auto & auto & auto & equal \\
\hline \multirow{3}{*}{5} & 2500 & 10 & Individual-based & $I_{H}^{1}$ & $\mathrm{~F}$ & 0.79 & auto & 0.43 & 0.31 & auto & 0.24 & auto & equal \\
\hline & 10000 & 30 & Population-based & $I_{H}^{1}$ & $\mathrm{~F}$ & 0.74 & 16 & 0.39 & 0.21 & auto & auto & auto & auto \\
\hline & 40000 & 30 & Individual-based & $I_{H}^{1}$ & $\mathrm{~F}$ & 0.62 & 17 & 0.21 & auto & 0.14 & auto & auto & equal \\
\hline \multirow{3}{*}{10} & 2500 & 20 & Population-based & $I_{H}^{1}$ & $\mathrm{~T}$ & 0.79 & 5 & 0.39 & 0.05 & 0.09 & 0.64 & auto & equal \\
\hline & 10000 & 20 & Population-based & $I_{H}^{1}$ & $\mathrm{~T}$ & 0.1 & auto & 0.36 & auto & auto & 0.5 & auto & 0.81 \\
\hline & 40000 & 20 & Individual-based & $I_{H}^{1}$ & $\mathrm{~T}$ & 0.16 & 18 & auto & 0.18 & auto & 0.73 & auto & auto \\
\hline
\end{tabular}

Table 2: Parameters selected by irace specific to MOEA/D.

\begin{tabular}{ccccccc}
\hline$M$ & $F E_{\max }$ & $\rho$ & $\delta$ & $\phi$ & $t_{\text {size }}$ & $v$ \\
\hline \multirow{3}{*}{2} & 2500 & 0.94 & 0.33 & 0.53 & 18 & 5 \\
\cline { 2 - 7 } & 10000 & 0.19 & 0.71 & 0.61 & 13 & 10 \\
\cline { 2 - 7 } & 40000 & 0.14 & 0.83 & 0.22 & 14 & 10 \\
\hline \multirow{3}{*}{3} & 2500 & 0.89 & 0.3 & 0.71 & 7 & 3 \\
\cline { 2 - 7 } & 10000 & 0.41 & 0.04 & 0.21 & 8 & 10 \\
\cline { 2 - 7 } & 40000 & 0.97 & 0.06 & 0.51 & 8 & 9 \\
\hline \multirow{3}{*}{5} & 2500 & 0.16 & 0.11 & 0.25 & 1 & 5 \\
\cline { 2 - 7 } 10 & 10000 & 0.8 & 0.08 & 0.12 & 18 & 9 \\
\cline { 2 - 7 } & 40000 & 0.74 & 0.35 & 0.57 & 11 & 10 \\
\hline \multirow{3}{*}{2500} & 0.87 & 0.84 & 0.64 & 15 & 2 \\
\cline { 2 - 7 } & 10000 & 0.42 & 0.07 & 0.98 & 17 & 9 \\
\cline { 2 - 7 } & 40000 & 0.92 & 0.07 & 0.05 & 13 & 7 \\
\hline
\end{tabular}


Table 3: Underlying EA parameters selected by irace for MOEAs. IBEA uses $I_{\epsilon+}$ for all scenarios.

\begin{tabular}{|c|c|c|c|c|c|c|c|c|c|c|c|c|}
\hline \multicolumn{4}{|c|}{ HypE } & \multicolumn{7}{|c|}{ GA } & \multicolumn{2}{|c|}{$\mathrm{DE}$} \\
\hline M & $F E_{\max }$ & $\mu$ & $\lambda_{r}$ & $t_{\text {size }}$ & $p_{c}$ & $p_{m}$ & bit & $p_{v}$ & $\eta_{c}$ & $\eta_{v}$ & $C R$ & $F$ \\
\hline \multirow{3}{*}{2} & 2500 & 20 & 0.11 & - & - & - & - & - & - & - & 0.48 & 0.76 \\
\hline & $\overline{10000}$ & 20 & 0.12 & - & - & - & - & - & - & - & 0.44 & 0.62 \\
\hline & 40000 & 50 & 0.10 & - & - & - & - & - & - & - & 0.53 & 0.35 \\
\hline \multirow{3}{*}{3} & 2500 & 20 & 0.10 & - & - & - & - & - & - & - & 0.28 & 0.51 \\
\hline & 10000 & 20 & 0.11 & - & - & - & - & - & - & - & 0.28 & 0.58 \\
\hline & 40000 & 20 & 0.11 & - & - & - & - & - & - & - & 0.31 & 0.63 \\
\hline \multirow{3}{*}{5} & 2500 & 10 & 0.11 & - & - & - & - & - & - & - & 0.26 & 0.85 \\
\hline & 10000 & 10 & 0.13 & - & - & - & - & - & - & - & 0.6 & 0.76 \\
\hline & 40000 & 20 & 0.12 & - & - & - & - & - & - & - & 0.23 & 0.71 \\
\hline \multirow{3}{*}{10} & 2500 & 40 & 0.11 & 4 & 0.94 & 0.37 & F & 0.6 & 26 & 49 & - & - \\
\hline & 10000 & 20 & 0.16 & - & - & - & - & - & - & - & 0.27 & 0.96 \\
\hline & 40000 & 40 & 0.24 & - & - & - & - & - & - & - & 0.21 & 0.49 \\
\hline \multicolumn{2}{|r|}{ IBEA } & & & \multicolumn{7}{|c|}{ GA } & \multicolumn{2}{|c|}{ DE } \\
\hline M & $F E_{\max }$ & $\mu$ & $\lambda_{r}$ & $t_{\text {size }}$ & $p_{c}$ & $p_{m}$ & bit & $p_{v}$ & $\eta_{c}$ & $\eta_{v}$ & $C R$ & $F$ \\
\hline \multirow{3}{*}{2} & 2500 & 20 & 1.01 & - & - & - & - & - & - & - & 0.39 & 0.65 \\
\hline & 10000 & 30 & 0.33 & - & - & - & - & - & - & - & 0.26 & 0.63 \\
\hline & 40000 & 60 & 0.16 & - & - & - & - & - & - & - & 0.36 & 0.35 \\
\hline \multirow{3}{*}{3} & 2500 & 30 & 0.2 & - & - & - & - & - & - & - & 0.27 & 0.35 \\
\hline & 10000 & 50 & 0.1 & - & - & - & - & - & - & - & 0.23 & 0.45 \\
\hline & 40000 & 60 & 0.19 & - & - & - & - & - & - & - & 0.32 & 0.35 \\
\hline \multirow{3}{*}{5} & 2500 & 20 & 0.8 & - & - & - & - & - & - & - & 0.25 & 0.56 \\
\hline & 10000 & 20 & 1.14 & - & - & - & - & - & - & - & 0.23 & 0.54 \\
\hline & 40000 & 70 & 0.89 & - & - & - & - & - & - & - & 0.17 & 0.3 \\
\hline \multirow{3}{*}{10} & 2500 & 40 & 0.86 & - & - & - & - & - & - & - & 0.35 & 0.35 \\
\hline & 10000 & 100 & 0.21 & - & - & - & - & - & - & - & 0.47 & 0.15 \\
\hline & 40000 & 100 & 0.75 & - & - & - & - & - & - & - & 0.7 & 0.13 \\
\hline
\end{tabular}

\begin{tabular}{|c|c|c|c|c|c|c|c|c|c|c|c|c|}
\hline \multicolumn{4}{|c|}{ MOEA/D } & \multicolumn{7}{|c|}{ GA } & \multicolumn{2}{|c|}{$\mathrm{DE}$} \\
\hline$M$ & $F E_{\max }$ & $\mu$ & $\lambda_{\text {agg }}$ & $t_{\text {size }}$ & $p_{c}$ & $p_{m}$ & bit & $p_{v}$ & $\eta_{c}$ & $\eta_{v}$ & $C R$ & $F$ \\
\hline \multirow{3}{*}{2} & 2500 & 100 & WS & 2 & 0.35 & 0.99 & F & 0.09 & 45 & 18 & - & - \\
\hline & 10000 & 100 & $\mathrm{TA}$ & 2 & 0.88 & 0.75 & F & 0.05 & 23 & 7 & - & - \\
\hline & 40000 & 200 & TA & 2 & 0.7 & 0.92 & $\mathrm{~F}$ & 0.04 & 47 & 16 & - & - \\
\hline \multirow{3}{*}{3} & 2500 & 200 & WS & 2 & 0.68 & 0.97 & F & 0.09 & 18 & 19 & - & - \\
\hline & 10000 & 100 & $\mathrm{TA}$ & - & - & - & - & - & - & - & 0.09 & 0.74 \\
\hline & 40000 & 400 & $\mathrm{TA}$ & 2 & 0.66 & 0.99 & $\mathrm{~T}$ & - & 32 & 8 & - & - \\
\hline \multirow{3}{*}{5} & 2500 & 300 & WS & 2 & 0.74 & 0.91 & $\mathrm{~T}$ & - & 13 & 3 & - & - \\
\hline & 10000 & 100 & TA & - & - & - & - & - & - & - & 0.12 & 0.79 \\
\hline & 40000 & 100 & TA & 2 & 0.64 & 0.94 & $\mathrm{~T}$ & - & 50 & 19 & - & - \\
\hline \multirow{3}{*}{10} & 2500 & 300 & TA & 2 & 0.91 & 0.97 & $\mathrm{~F}$ & 0.07 & 46 & 4 & - & - \\
\hline & 10000 & 300 & $\mathrm{TA}$ & 2 & 0.95 & 0.85 & $\mathrm{~T}$ & - & 7 & 5 & - & - \\
\hline & 40000 & 200 & $\mathrm{TA}$ & 2 & 0.8 & 0.97 & $\mathrm{~T}$ & - & 35 & 7 & - & - \\
\hline \multicolumn{2}{|r|}{ MOGA } & & \multicolumn{8}{|c|}{ GA } & \multicolumn{2}{|c|}{$\mathrm{DE}$} \\
\hline$M$ & $F E_{\max }$ & $\mu$ & $\sigma_{0}$ & $t_{\text {size }}$ & $p_{c}$ & $p_{m}$ & bit & $p_{v}$ & $\eta_{c}$ & $\eta_{v}$ & $C R$ & $F$ \\
\hline \multirow{3}{*}{2} & 2500 & 100 & 0.46 & 8 & 0.62 & 0.36 & $\mathrm{~F}$ & 0.57 & 41 & 18 & - & - \\
\hline & 10000 & 100 & 0.52 & 4 & 0.43 & 0.19 & $\mathrm{~F}$ & 0.35 & 49 & 28 & - & - \\
\hline & 40000 & 100 & 0.86 & 4 & 0.29 & 0.29 & $\mathrm{~F}$ & 0.05 & 25 & 9 & - & - \\
\hline \multirow{3}{*}{3} & 2500 & 100 & 0.84 & 4 & 0.63 & 0.27 & $\mathrm{~F}$ & 0.39 & 41 & 36 & - & - \\
\hline & 10000 & 100 & 0.82 & 4 & 0.66 & 0.26 & $\mathrm{~F}$ & 0.43 & 40 & 39 & - & - \\
\hline & 40000 & 100 & 1.00 & 4 & 0.57 & 0.39 & $\mathrm{~T}$ & - & 40 & 10 & - & - \\
\hline \multirow{3}{*}{ 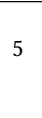 } & 2500 & 20 & - & - & - & - & - & - & - & - & 0.99 & 0.68 \\
\hline & 10000 & 20 & - & - & - & - & - & - & - & - & 1.00 & 0.69 \\
\hline & 40000 & 10 & 0.42 & 8 & 0.07 & 0.13 & $\mathrm{~T}$ & - & 13 & 8 & - & - \\
\hline \multirow{3}{*}{10} & 2500 & 60 & 0.79 & 8 & 0.58 & 0.14 & $\mathrm{~F}$ & 0.97 & 23 & 4 & - & - \\
\hline & 10000 & 100 & 0.87 & 4 & 0.72 & 0.24 & $\mathrm{~F}$ & 0.39 & 24 & 3 & - & - \\
\hline & 40000 & 80 & 0.57 & 4 & 0.36 & 0.5 & $\mathrm{~T}$ & - & 40 & 3 & - & - \\
\hline
\end{tabular}


Table 4: Underlying EA parameters selected by irace for MOEAs. SPEA2 uses $t_{\text {size }}=8$ when $M<10, t_{\text {size }}=2$ on $\langle 10,10\rangle$, and $t_{\text {size }}=4$ on $\langle 10,40\rangle$. NSGA-III uses $H_{2}=2$ on all $M=10$ scenarios but $\langle 10,40\rangle$, where $H_{2}=1$

\begin{tabular}{|c|c|c|c|c|c|c|c|c|c|c|c|c|}
\hline \multicolumn{4}{|c|}{ NSGA-II } & \multicolumn{7}{|c|}{ GA } & \multicolumn{2}{|c|}{$\mathrm{DE}$} \\
\hline M & $F E_{\max }$ & $\mu$ & $\lambda_{r}$ & $t_{\text {size }}$ & $p_{c}$ & $p_{m}$ & bit & $p_{v}$ & $\eta_{c}$ & $\eta_{v}$ & $C R$ & $F$ \\
\hline \multirow{3}{*}{2} & 2500 & 10 & 0.15 & 8 & 0.28 & 0.58 & F & 0.04 & 34 & 7 & - & - \\
\hline & 10000 & 40 & 1.25 & - & - & - & - & - & - & - & 0.37 & 0.35 \\
\hline & 40000 & 60 & 0.84 & - & - & - & - & - & - & - & 0.24 & 0.36 \\
\hline \multirow{3}{*}{3} & 2500 & 10 & 0.11 & - & - & - & - & - & - & - & 0.12 & 0.78 \\
\hline & 10000 & 20 & 0.11 & 8 & 0.26 & 0.66 & $\mathrm{~T}$ & - & 38 & 8 & - & - \\
\hline & 40000 & 60 & 1.16 & 8 & 0.12 & 0.14 & $\mathrm{~T}$ & - & 35 & 19 & - & - \\
\hline \multirow{3}{*}{5} & 2500 & 10 & 0.14 & - & - & - & - & - & - & - & 0.13 & 0.75 \\
\hline & 10000 & 10 & 0.13 & 4 & 0.66 & 0.86 & $\mathrm{~T}$ & - & 20 & 7 & - & - \\
\hline & 40000 & 20 & 1.18 & 4 & 0.13 & 0.32 & $\mathrm{~T}$ & - & 35 & 7 & - & - \\
\hline \multirow{3}{*}{10} & 2500 & 70 & 0.29 & 8 & 0.9 & 0.37 & $\mathrm{~F}$ & 0.42 & 44 & 5 & - & - \\
\hline & 10000 & 50 & 0.4 & 4 & 0.35 & 0.29 & $\mathrm{~T}$ & - & 47 & 2 & - & - \\
\hline & 40000 & 30 & 1.29 & - & - & - & - & - & - & - & 0.08 & 0.46 \\
\hline \multicolumn{2}{|c|}{ NSGA-III } & & & \multicolumn{7}{|c|}{ GA } & \multicolumn{2}{|c|}{$\mathrm{DE}$} \\
\hline M & $F E_{\max }$ & $\mu^{\prime}$ & $H_{1}$ & $t_{\text {size }}$ & $p_{c}$ & $p_{m}$ & bit & $p_{v}$ & $\eta_{c}$ & $\eta_{v}$ & $C R$ & $F$ \\
\hline \multirow{3}{*}{2} & 2500 & 0.65 & 62 & - & - & - & - & - & - & - & 0.98 & 0.33 \\
\hline & 10000 & 1.35 & 2 & 4 & 0.6 & 0.93 & $\mathrm{~T}$ & - & 27 & 4 & - & - \\
\hline & 40000 & 1.15 & 4 & 4 & 0.73 & 0.82 & $\mathrm{~T}$ & - & 1 & 14 & - & - \\
\hline \multirow{3}{*}{3} & 2500 & 0.72 & 8 & - & - & - & - & - & - & - & 0.98 & 0.4 \\
\hline & 10000 & 1.02 & 2 & 4 & 0.61 & 0.91 & $\mathrm{~T}$ & - & 9 & 3 & - & - \\
\hline & 40000 & 1.36 & 4 & 4 & 0.79 & 0.9 & $\mathrm{~T}$ & - & 43 & 8 & - & - \\
\hline \multirow{3}{*}{5} & 2500 & 1.19 & 2 & 4 & 0.92 & 0.46 & $\mathrm{~F}$ & 0.14 & 29 & 3 & - & - \\
\hline & 10000 & 1.15 & 2 & 4 & 0.89 & 0.84 & F & 0.06 & 17 & 8 & - & - \\
\hline & 40000 & 1.36 & 2 & 4 & 0.73 & 0.94 & $\mathrm{~T}$ & - & 42 & 4 & - & - \\
\hline \multirow{3}{*}{10} & 2500 & 0.84 & 2 & 4 & 0.89 & 0.45 & F & 0.55 & 18 & 18 & - & - \\
\hline & 10000 & 0.85 & 2 & 4 & 1.00 & 0.58 & $\mathrm{~T}$ & - & 34 & 5 & - & - \\
\hline & 40000 & 0.56 & 3 & 4 & 0.92 & 0.62 & $\mathrm{~T}$ & - & 36 & 1 & - & - \\
\hline
\end{tabular}

\begin{tabular}{|c|c|c|c|c|c|c|c|c|c|c|c|c|}
\hline \multicolumn{4}{|c|}{ SMS } & \multicolumn{7}{|c|}{ GA } & \multicolumn{2}{|c|}{$\mathrm{DE}$} \\
\hline$M$ & $1 \quad F E_{\max }$ & $\mu$ & $\lambda_{r}$ & $t_{\text {size }}$ & $p_{c}$ & $p_{m}$ & bit & $p_{v}$ & $\eta_{c}$ & $\eta_{v}$ & $C R$ & $F$ \\
\hline \multirow{3}{*}{2} & 2500 & 20 & - & - & - & - & - & - & - & - & 0.52 & 0.56 \\
\hline & 10000 & 60 & - & - & - & - & - & - & - & - & 0.36 & 0.42 \\
\hline & 40000 & 100 & - & - & - & - & - & - & - & - & 0.49 & 0.25 \\
\hline \multirow{3}{*}{3} & 2500 & 20 & - & - & - & - & - & - & - & - & 0.22 & 0.49 \\
\hline & 10000 & 40 & - & - & - & - & - & - & - & - & 0.26 & 0.39 \\
\hline & 40000 & 60 & - & - & - & - & - & - & - & - & 0.12 & 0.56 \\
\hline \multirow{3}{*}{5} & 2500 & 20 & - & - & - & - & - & - & - & - & 0.22 & 0.53 \\
\hline & 10000 & 30 & - & - & - & - & - & - & - & - & 0.17 & 0.5 \\
\hline & 40000 & 40 & - & - & - & - & - & - & - & - & 0.18 & 0.4 \\
\hline \multirow{3}{*}{10} & 2500 & 40 & - & - & - & - & - & - & - & - & 0.33 & 0.68 \\
\hline & 10000 & 30 & - & - & - & - & - & - & - & - & 0.19 & 0.84 \\
\hline & 40000 & 10 & - & - & 0.18 & 0.89 & $\mathrm{~T}$ & - & 38 & 2 & - & - \\
\hline \multicolumn{2}{|r|}{ SPEA2 } & & & \multicolumn{7}{|c|}{ GA } & \multicolumn{2}{|c|}{$\mathrm{DE}$} \\
\hline$M$ & $1 \quad F E_{\max }$ & $\mu$ & $\lambda_{r}$ & $k$ & $p_{c}$ & $p_{m}$ & bit & $p_{v}$ & $\eta_{c}$ & $\eta_{v}$ & $C R$ & $F$ \\
\hline \multirow{3}{*}{2} & 2500 & 10 & 0.18 & auto & 0.14 & 0.3 & $\mathrm{~T}$ & - & 11 & 5 & - & - \\
\hline & 10000 & 40 & 0.34 & - & - & - & - & - & - & - & 0.41 & 0.38 \\
\hline & 40000 & 60 & 0.33 & - & - & - & - & - & - & - & 0.45 & 0.3 \\
\hline \multirow{3}{*}{3} & 2500 & 10 & 0.49 & - & - & - & - & - & - & - & 0.11 & 0.6 \\
\hline & 10000 & 30 & 1.74 & 4 & 0.05 & 0.11 & $\mathrm{~T}$ & - & 37 & 10 & - & - \\
\hline & 40000 & 40 & 0.42 & auto & 0.17 & 0.21 & $\mathrm{~T}$ & - & 37 & 5 & - & - \\
\hline \multirow{3}{*}{5} & 2500 & 10 & 0.39 & - & - & - & - & - & - & - & 0.03 & 0.72 \\
\hline & 10000 & 20 & 0.22 & - & - & - & - & - & - & - & 0.03 & 0.54 \\
\hline & 40000 & 30 & 0.11 & - & - & - & - & - & - & - & 0.06 & 0.54 \\
\hline \multirow{3}{*}{10} & 2500 & 80 & 1.02 & 4 & 0.42 & 0.11 & $\mathrm{~T}$ & - & 39 & 30 & - & - \\
\hline & 10000 & 100 & 1.28 & 2 & 0.61 & 0.24 & $\mathrm{~F}$ & 0.06 & 29 & 12 & - & - \\
\hline & 40000 & 100 & 1.25 & - & - & - & - & - & - & & 0.03 & 0.10 \\
\hline
\end{tabular}




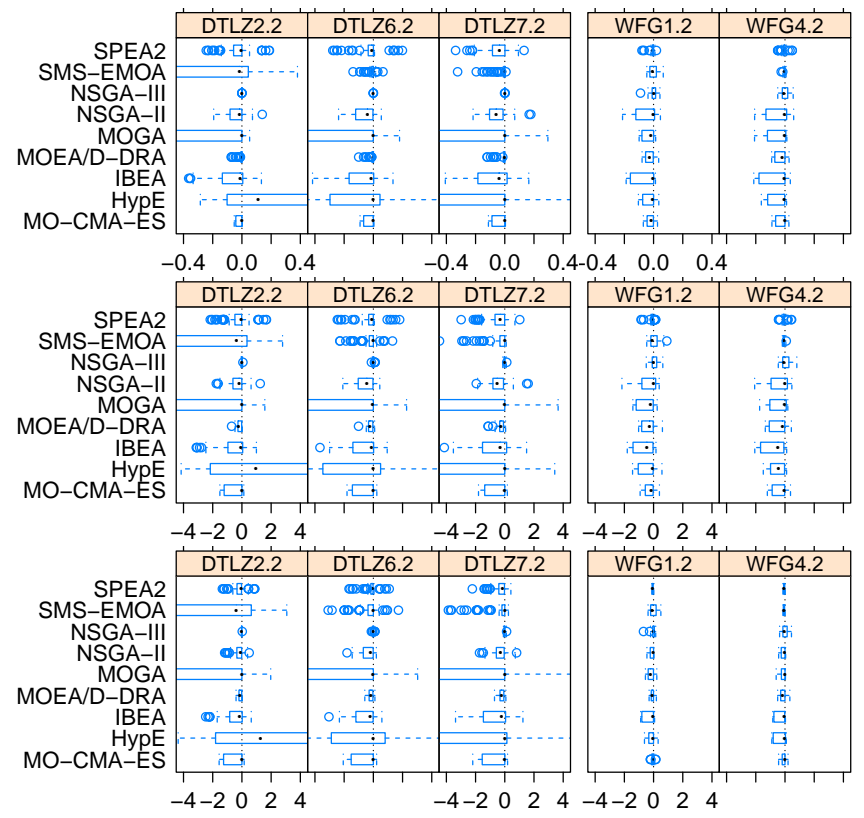

Figure 1: Performance differences between runs from MOEAs with and without unbounded archiving on the scenario with $M=2$ and $\boldsymbol{F} \boldsymbol{E}_{\max }=2500$ for selected problems with $n_{\mathrm{var}}=40$. From top to bottom, $I_{H}^{\text {rd }}, I_{\epsilon+}$, and $I_{I G D}$.

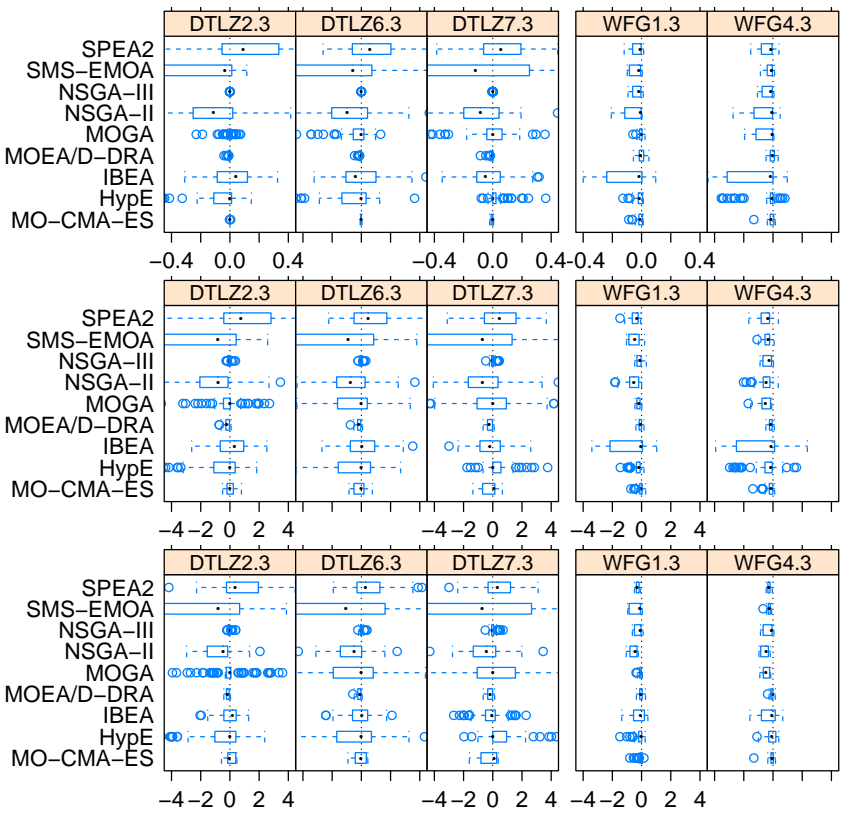

Figure 2: Performance differences between runs from MOEAs with and without unbounded archiving on the scenario with $M=3$ and $\boldsymbol{F} \boldsymbol{E}_{\text {max }}=2500$ for selected problems with $n_{\mathrm{var}}=40$. From top to bottom, $I_{H}^{r d}, I_{\epsilon+}$, and $I_{I G D}$.

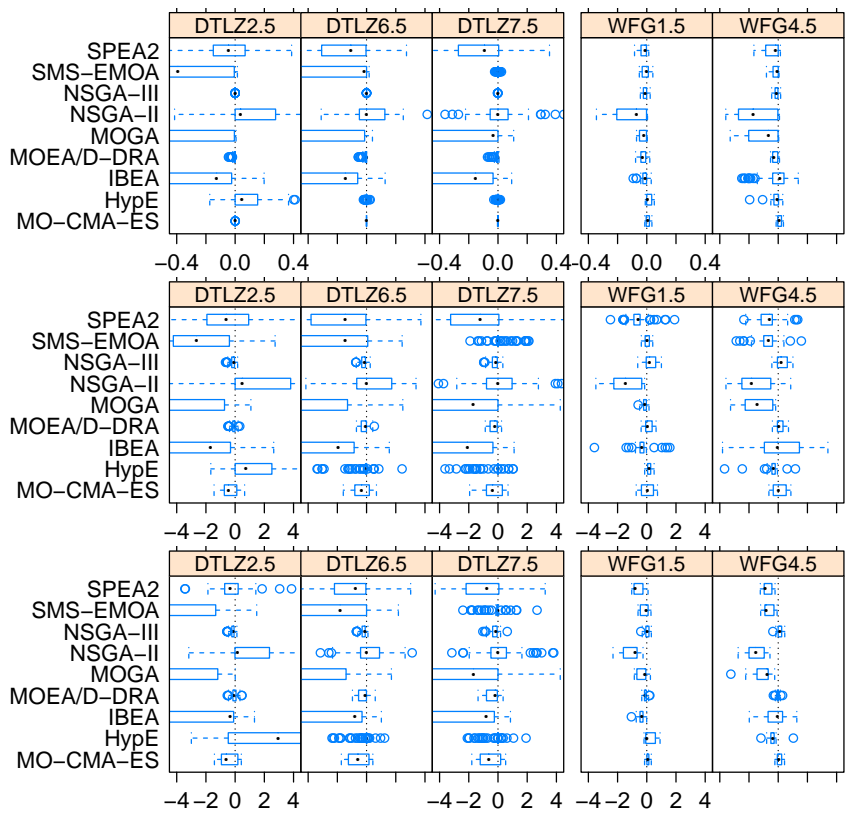

Figure 3: Performance differences between runs from MOEAs with and without unbounded archiving on the scenario with $M=5$ and $\boldsymbol{F} E_{\max }=2500$ for selected problems with $n_{\mathrm{var}}=40$. From top to bottom, $I_{H}^{r d}, I_{\epsilon+}$, and $I_{I G D}$.

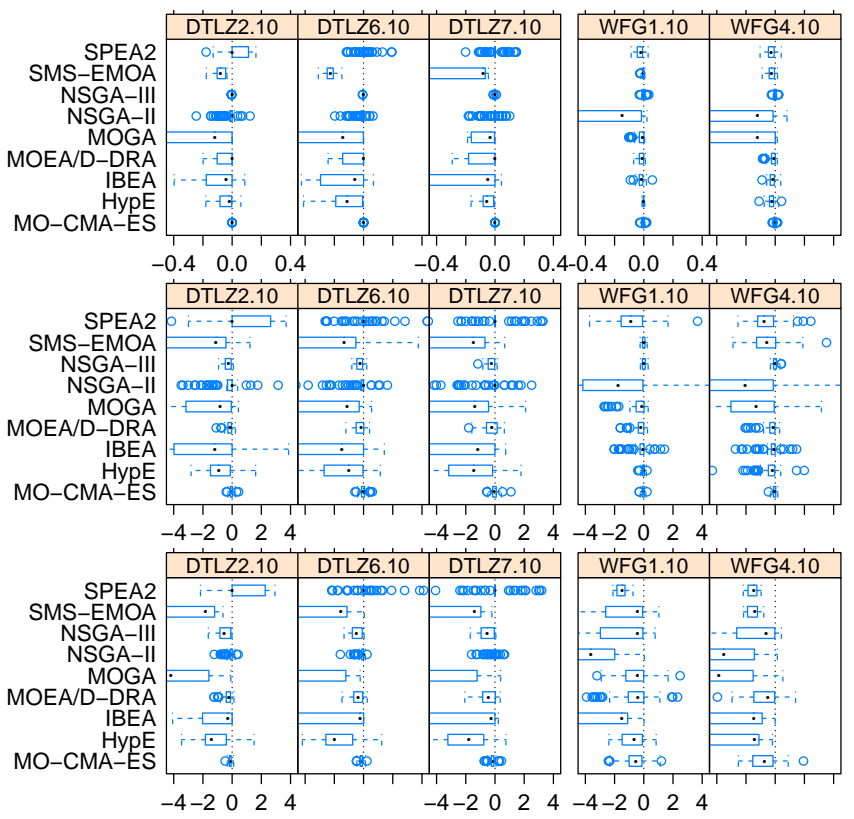

Figure 4: Performance differences between runs from MOEAs with and without unbounded archiving on the scenario with $M=10$ and $\boldsymbol{F} \boldsymbol{E}_{\max }=2500$ for selected problems with $n_{\mathrm{var}}=40$. From top to bottom, $I_{H}^{r d}, I_{\epsilon+}$, and $I_{I G D}$. 


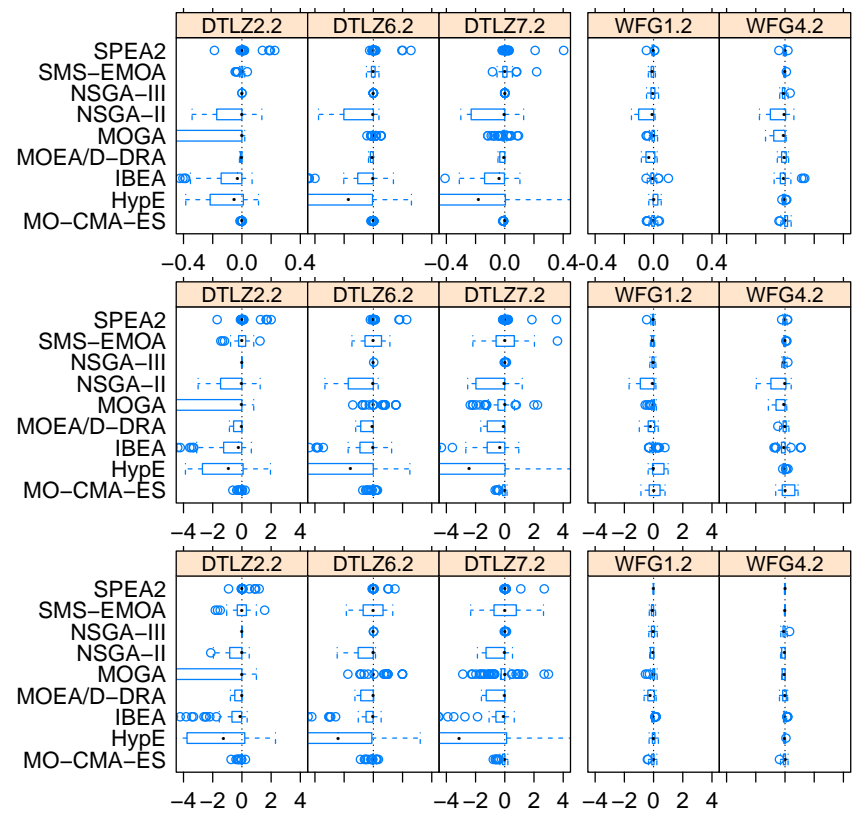

Figure 5: Performance differences between runs from MOEAs with and without unbounded archiving on the scenario with $M=2$ and $\boldsymbol{F} \boldsymbol{E}_{\max }=10000$ for selected problems with $n_{\mathrm{var}}=40$. From top to bottom, $I_{H}^{r d}, I_{\epsilon+}$, and $I_{I G D}$.

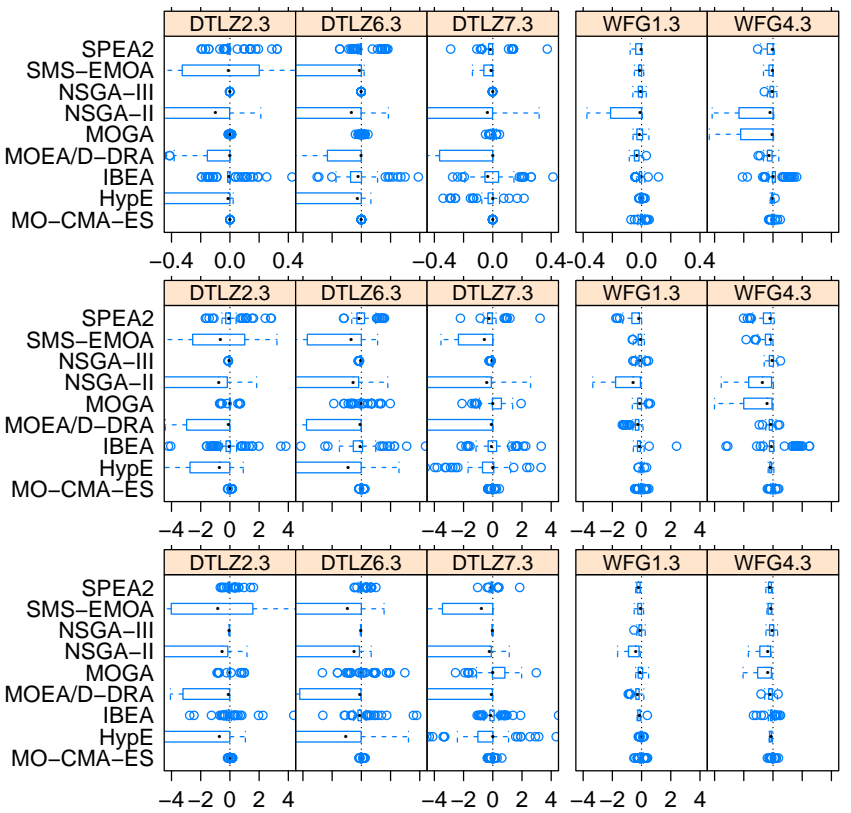

Figure 6: Performance differences between runs from MOEAs with and without unbounded archiving on the scenario with $M=3$ and $\boldsymbol{F} E_{\max }=10000$ for selected problems with $n_{\mathrm{var}}=40$. From top to bottom, $I_{H}^{r d}, I_{\epsilon+}$, and $I_{I G D}$.

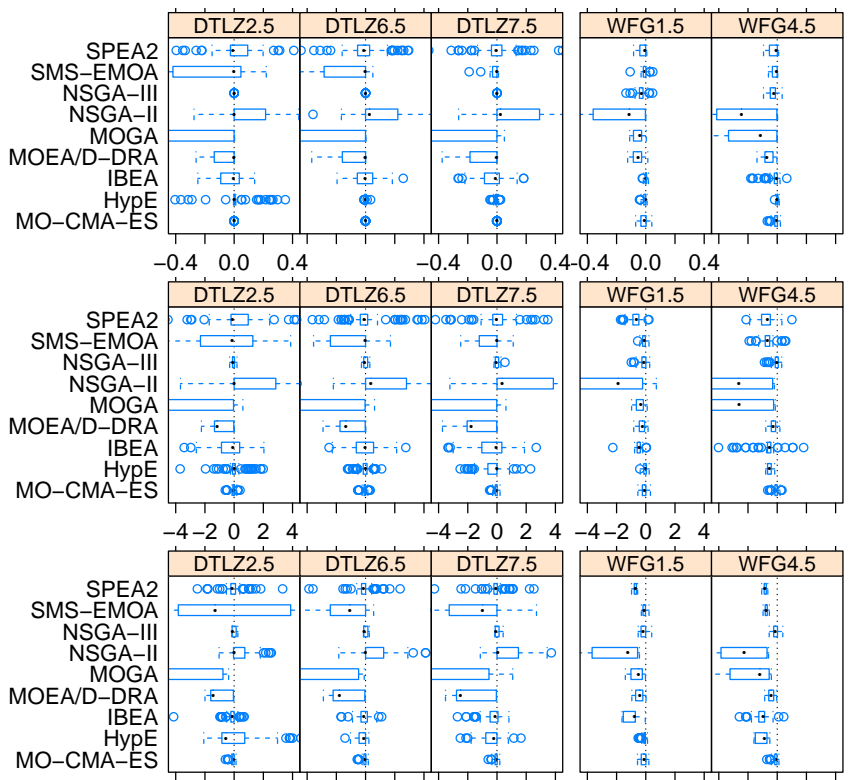

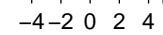

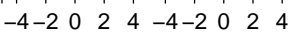

Figure 7: Performance differences between runs from MOEAs with and without unbounded archiving on the scenario with $M=5$ and $\boldsymbol{F} \boldsymbol{E}_{\max }=10000$ for selected problems with $n_{\mathrm{var}}=40$. From top to bottom, $I_{H}^{r d}, I_{\epsilon+}$, and $I_{I G D}$.

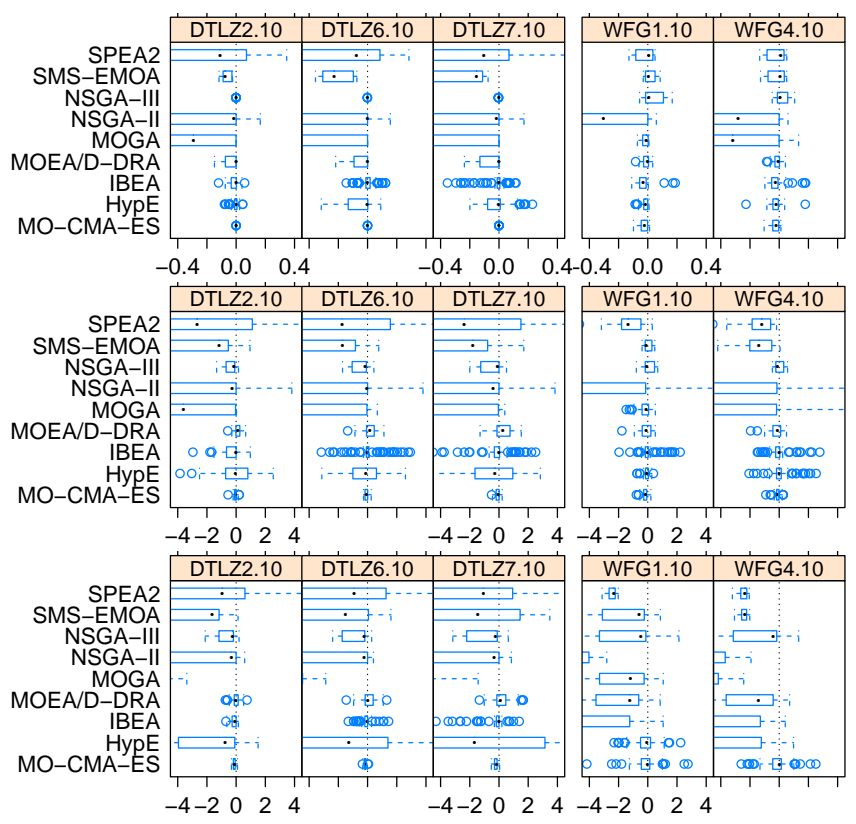

Figure 8: Performance differences between runs from MOEAs with and without unbounded archiving on the scenario with $M=10$ and $\boldsymbol{F} \boldsymbol{E}_{\max }=10000$ for selected problems with $n_{\mathrm{var}}=40$. From top to bottom, $I_{H}^{r d}, I_{\epsilon+}$, and $I_{I G D}$. 


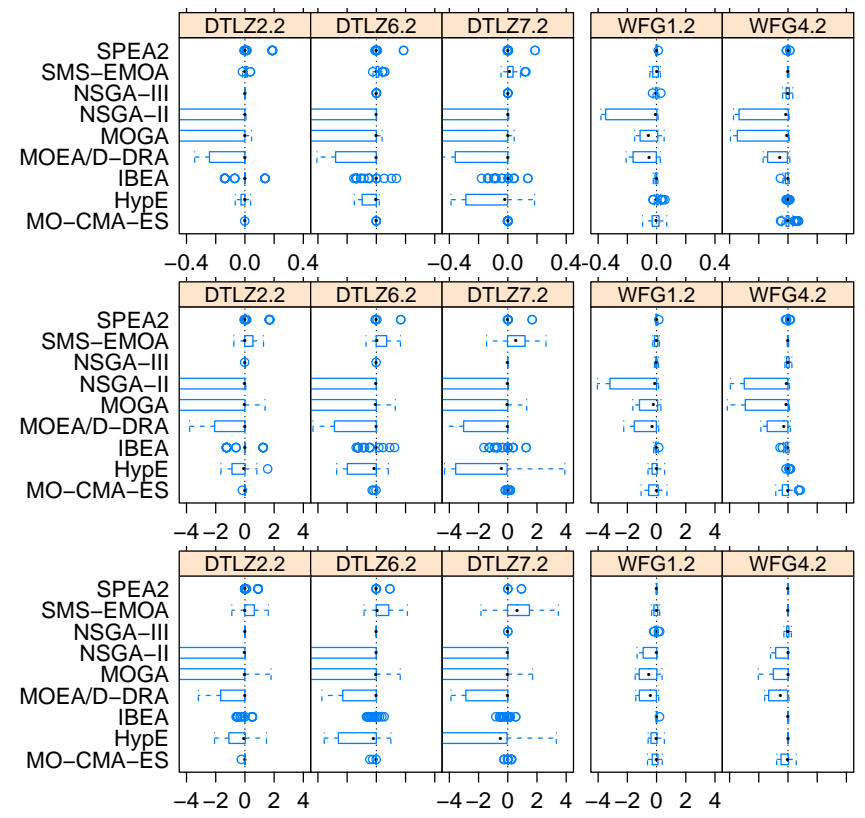

Figure 9: Performance differences between runs from MOEAs with and without unbounded archiving on the scenario with $M=2$ and $\boldsymbol{F} \boldsymbol{E}_{\max }=40000$ for selected problems with $n_{\mathrm{var}}=40$. From top to bottom, $I_{H}^{\text {rd }}, I_{\epsilon+}$, and $I_{I G D}$.

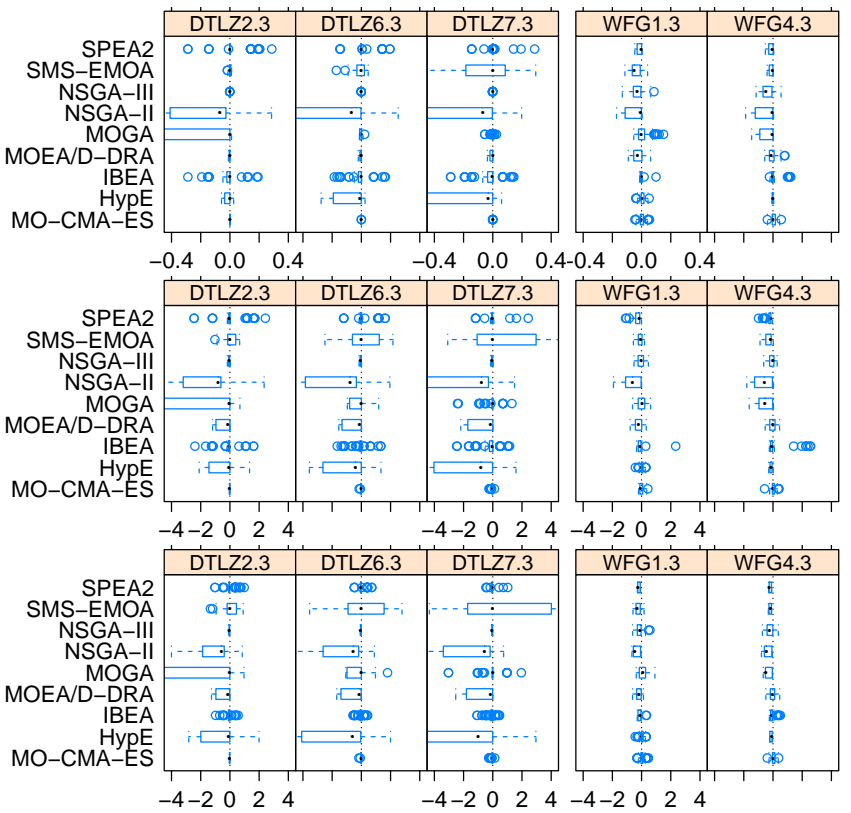

Figure 10: Performance differences between runs from MOEAs with and without unbounded archiving on the scenario with $M=3$ and $F E_{\max }=40000$ for selected problems with $n_{\mathrm{var}}=40$. From top to bottom, $I_{H}^{r d}, I_{\epsilon+}$, and $I_{I G D}$.

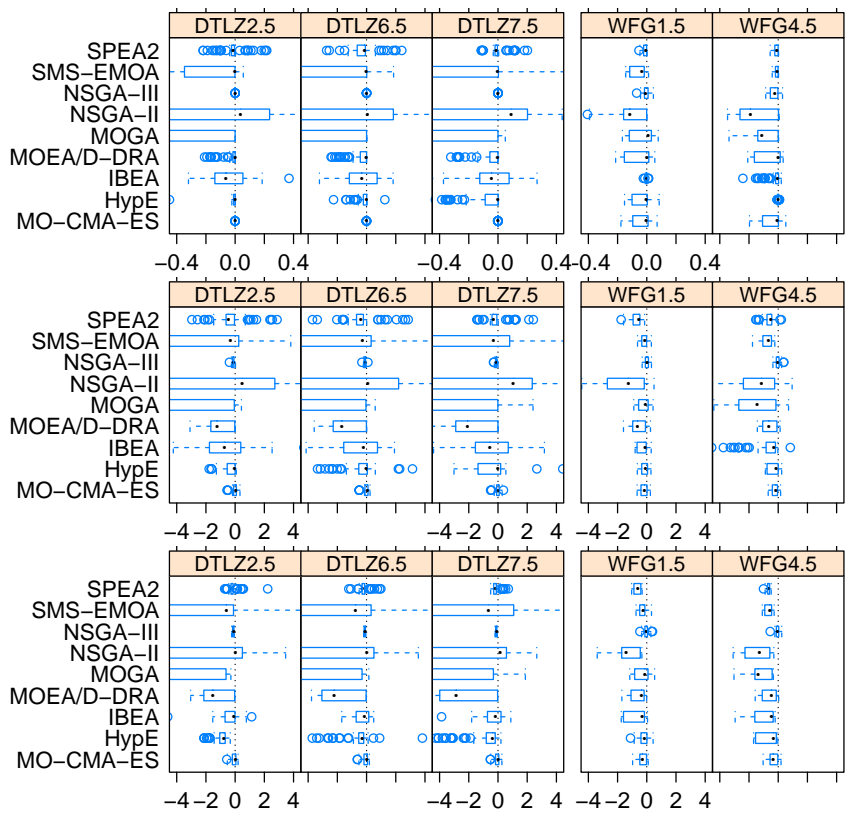

Figure 11: Performance differences between runs from MOEAs with and without unbounded archiving on the scenario with $M=5$ and $\boldsymbol{F} \boldsymbol{E}_{\max }=40000$ for selected problems with $n_{\mathrm{var}}=40$. From top to bottom, $I_{H}^{r d}, I_{\epsilon+}$, and $I_{I G D}$.

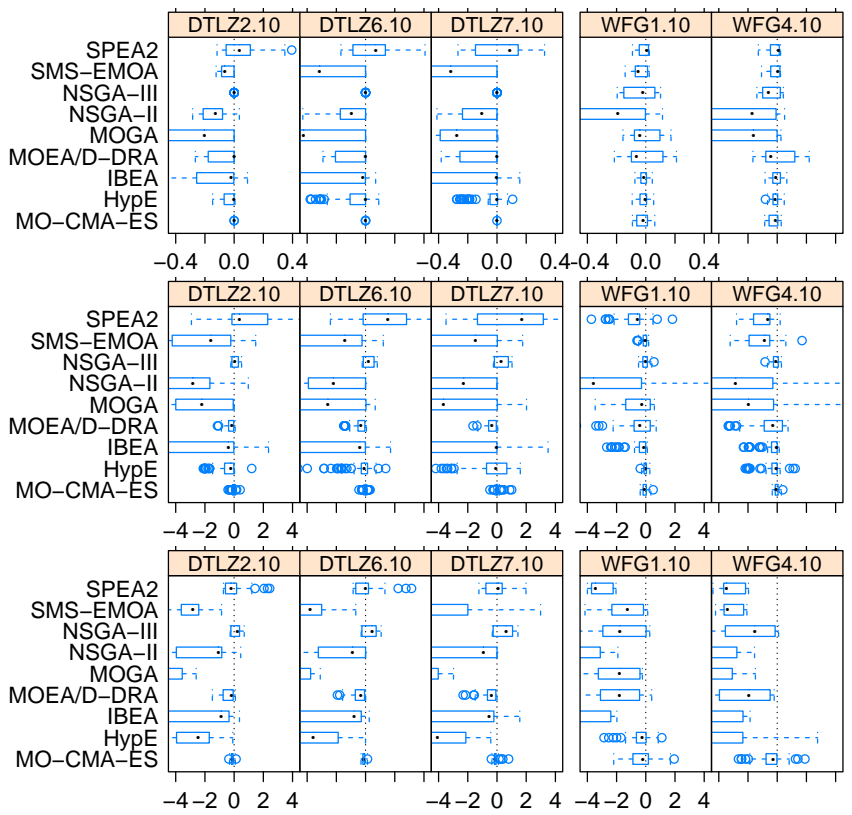

Figure 12: Performance differences between runs from MOEAs with and without unbounded archiving on the scenario with $M=10$ and $\boldsymbol{F} E_{\max }=40000$ for selected problems with $n_{\mathrm{var}}=40$. From top to bottom, $I_{H}^{r d}, I_{\epsilon+}$, and $I_{I G D}$. 

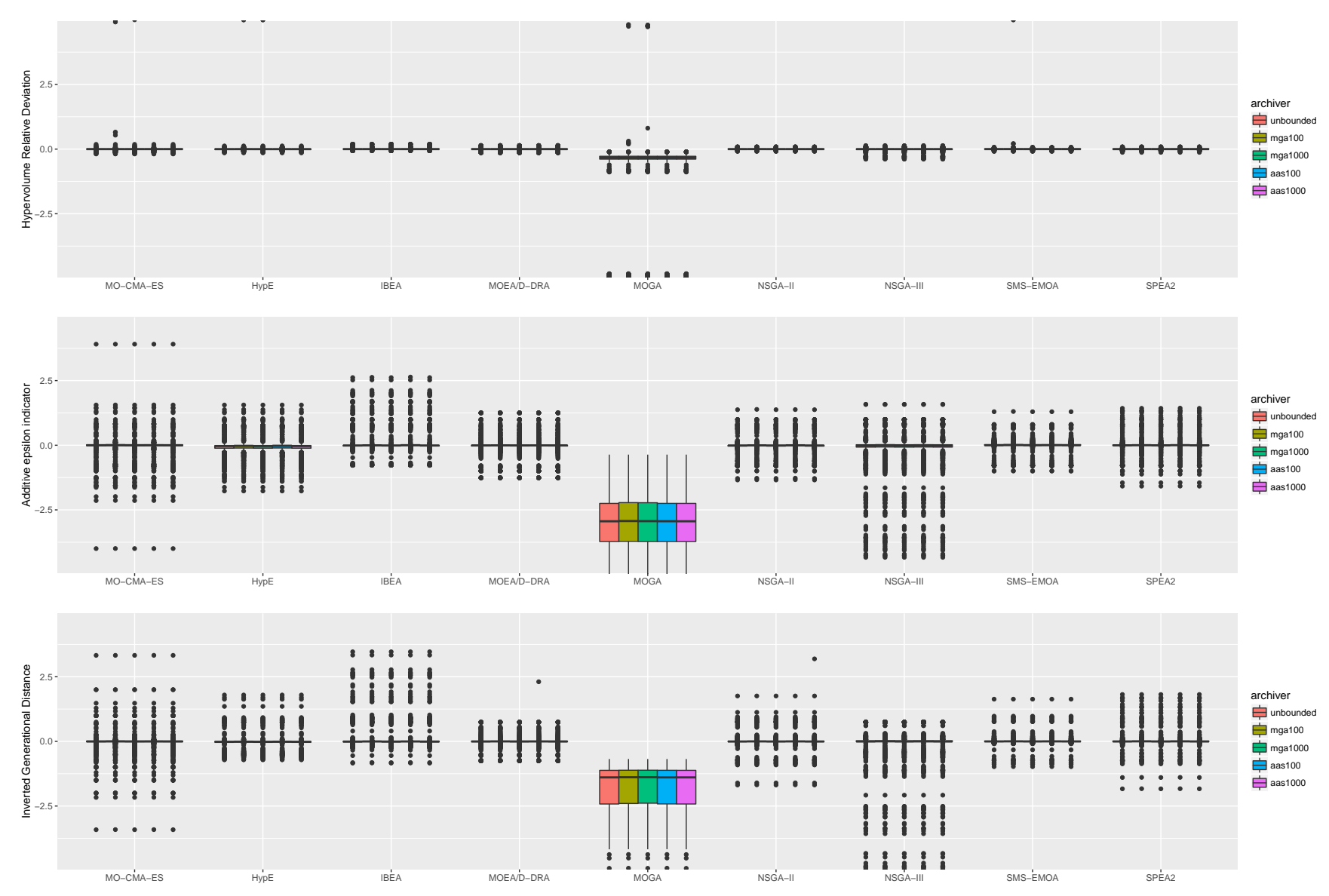

Figure 13: Boxplots of performance differences for $M=2$ and $F E_{\max }=40000$, grouped by MOEA and benchmark function, depicting the different archiving alternatives. From top to bottom: $I_{H}^{r d}, I_{\epsilon+}$, and $I_{I G D}$. 

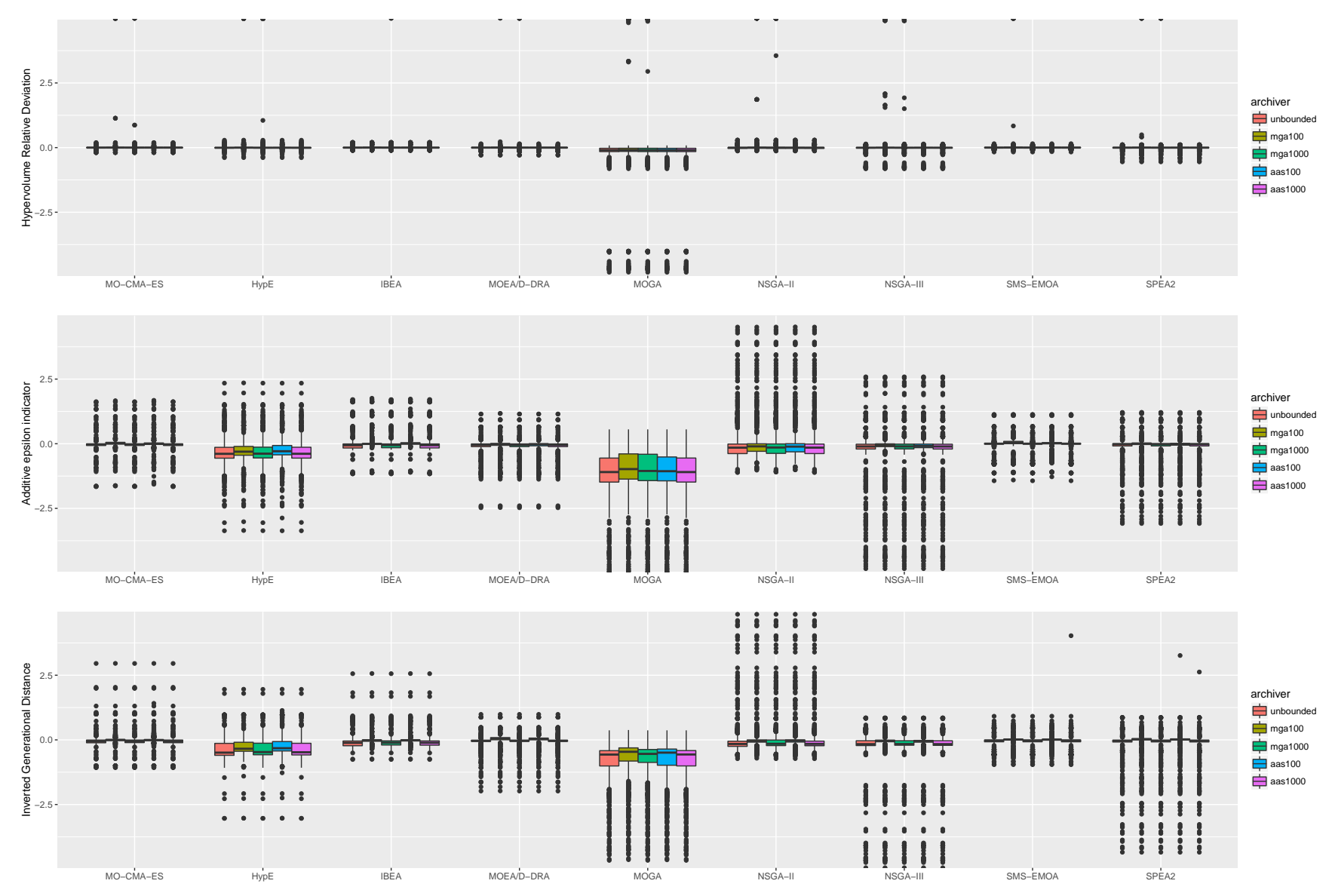

Figure 14: Boxplots of performance differences for $M=3$ and $\boldsymbol{F} E_{\max }=40000$, grouped by MOEA and benchmark function, depicting the different archiving alternatives. From top to bottom: $I_{H}^{r d}, I_{\epsilon+}$, and $I_{I G D}$. 


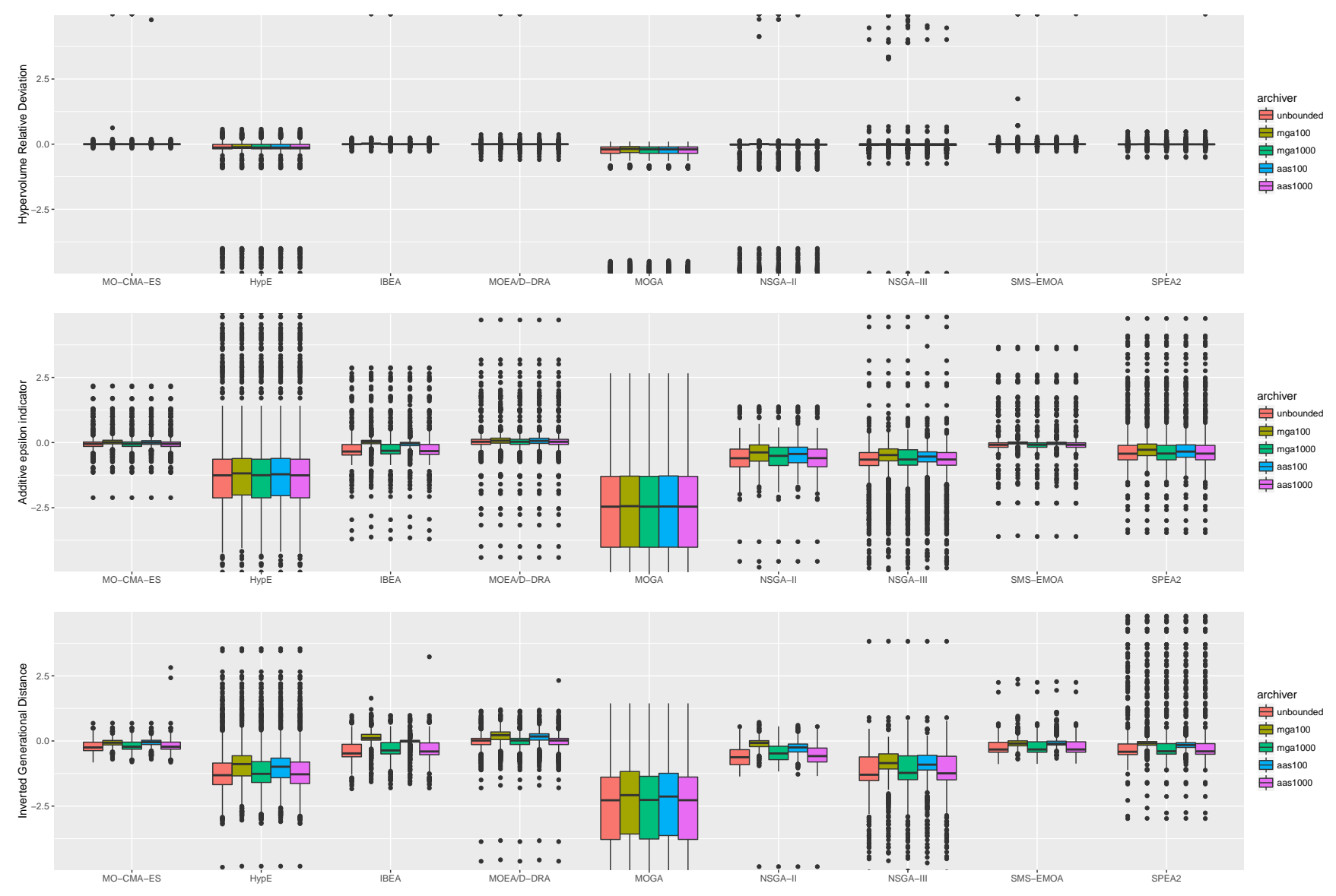

Figure 15: Boxplots of performance differences for $M=5$ and $F E_{\max }=40000$, grouped by MOEA and benchmark function, depicting the different archiving alternatives. From top to bottom: $I_{H}^{r d}, I_{\epsilon+}$, and $I_{I G D}$. 

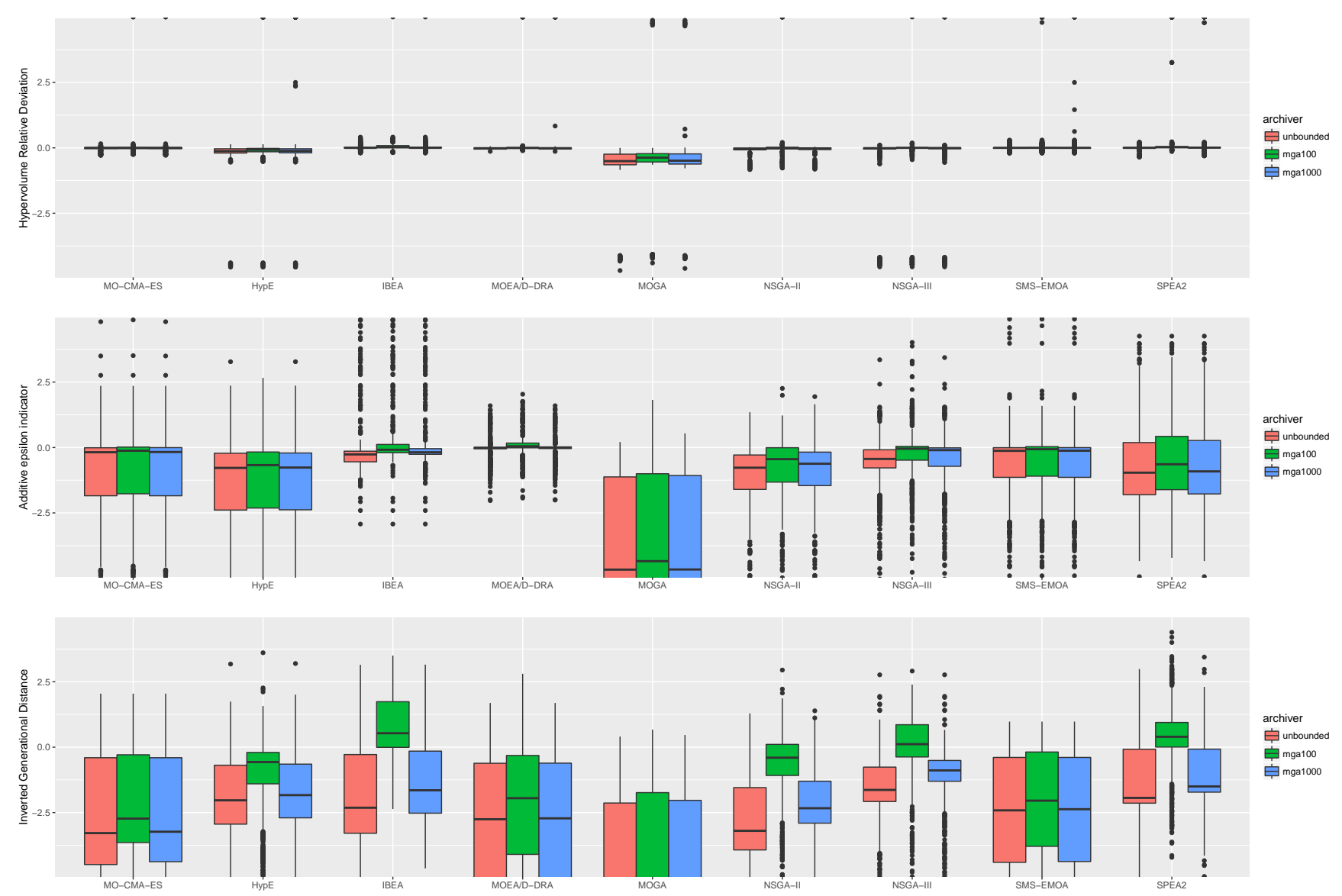

Figure 16: Boxplots of performance differences for $M=10$ and $\boldsymbol{F} E_{\max }=40000$, grouped by MOEA and benchmark function, depicting the different archiving alternatives. From top to bottom: $I_{H}^{r d}, I_{\epsilon+}$, and $I_{I G D}$. 

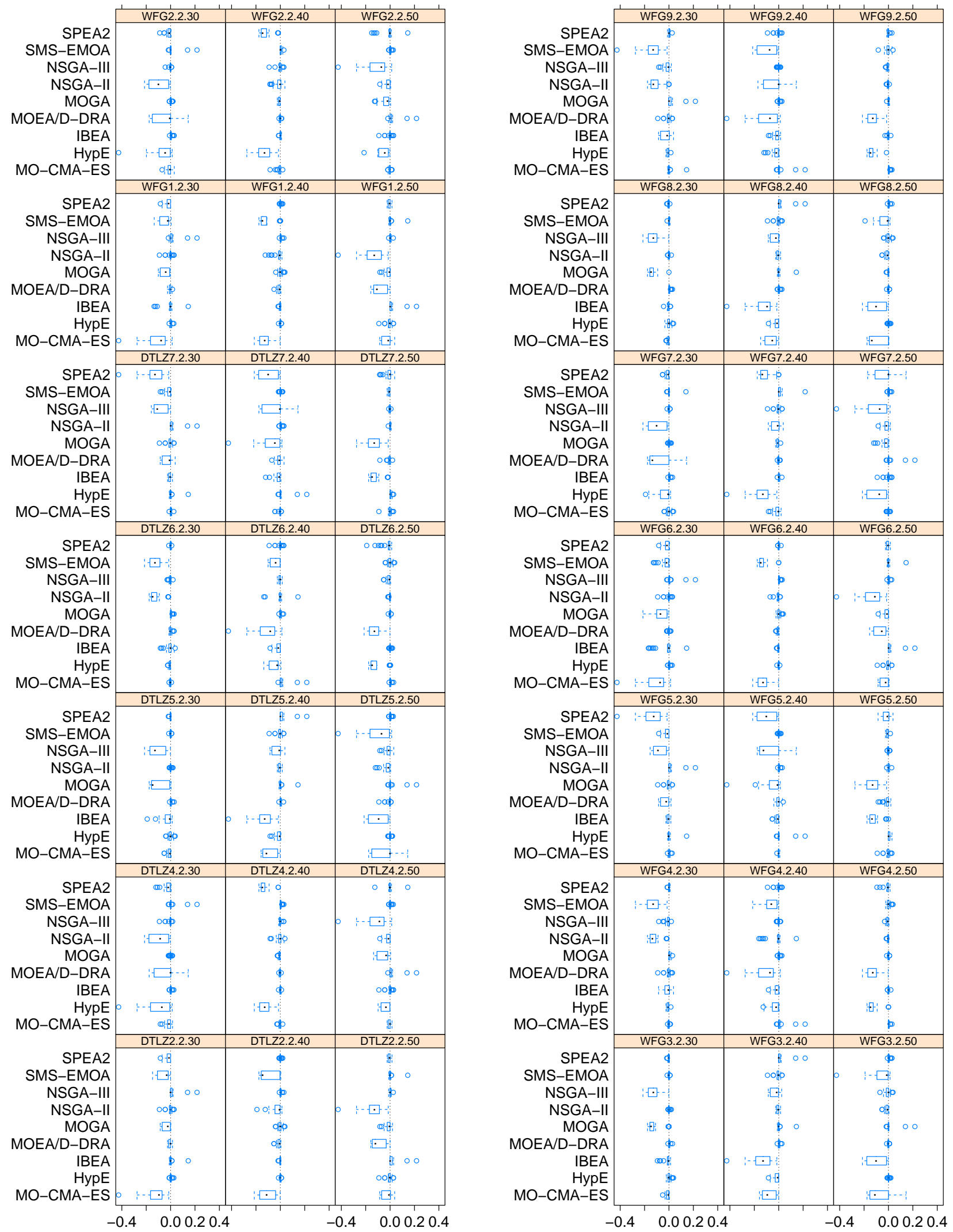

Figure 17: Performance differences according to $I_{H}^{\text {rd }}$ between runs from MOEAs with and without unbounded archiving on the scenario with $M=2$ and $\boldsymbol{F} E_{\max }=2500$ for all problems with $n_{\mathrm{var}} \in\{30,40,50\}$. 

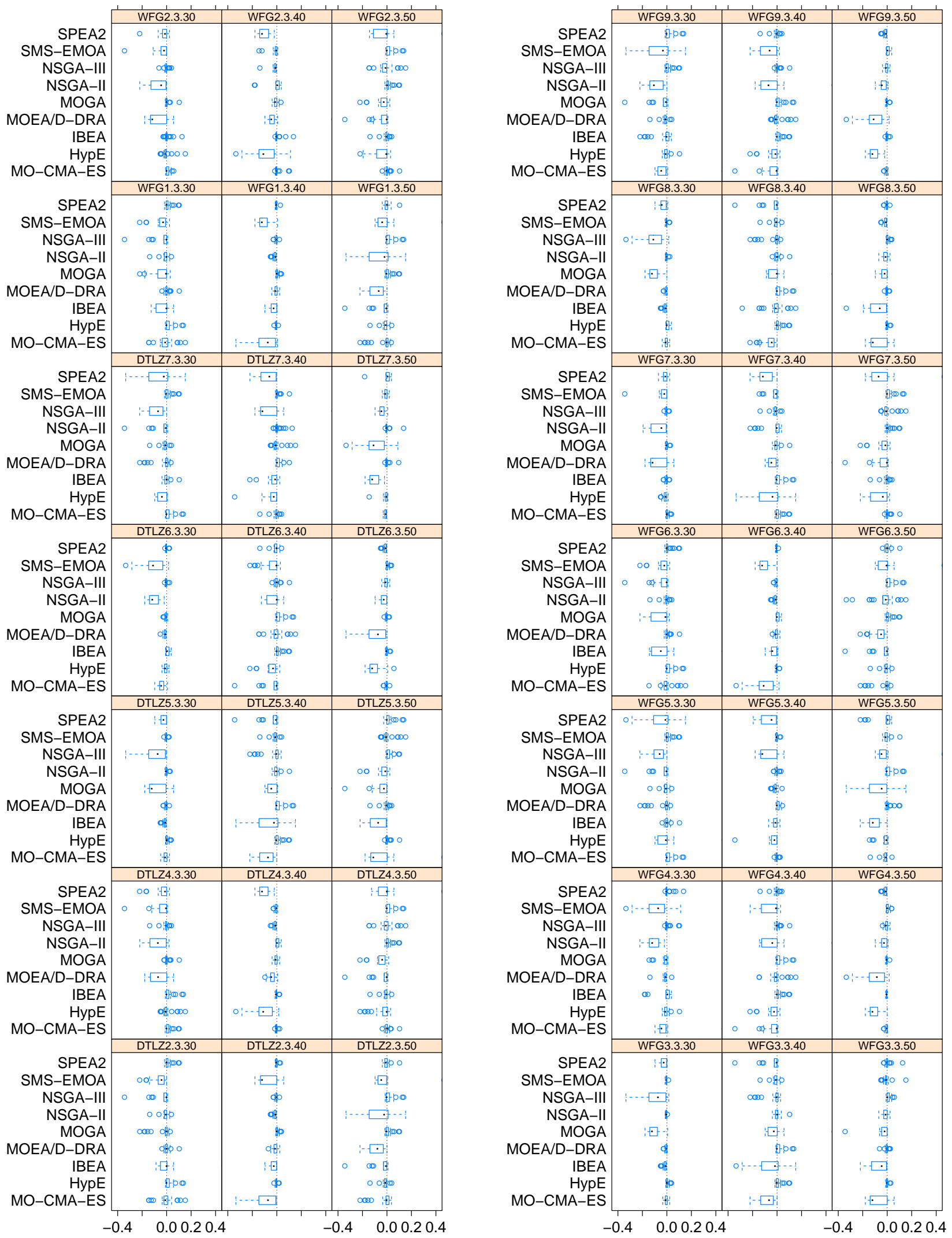

Figure 18: Performance differences according to $I_{H}^{\text {rd }}$ between runs from MOEAs with and without unbounded archiving on the scenario with $M=3$ and $\boldsymbol{F} E_{\max }=2500$ for all problems with $n_{\text {var }} \in\{30,40,50\}$. 

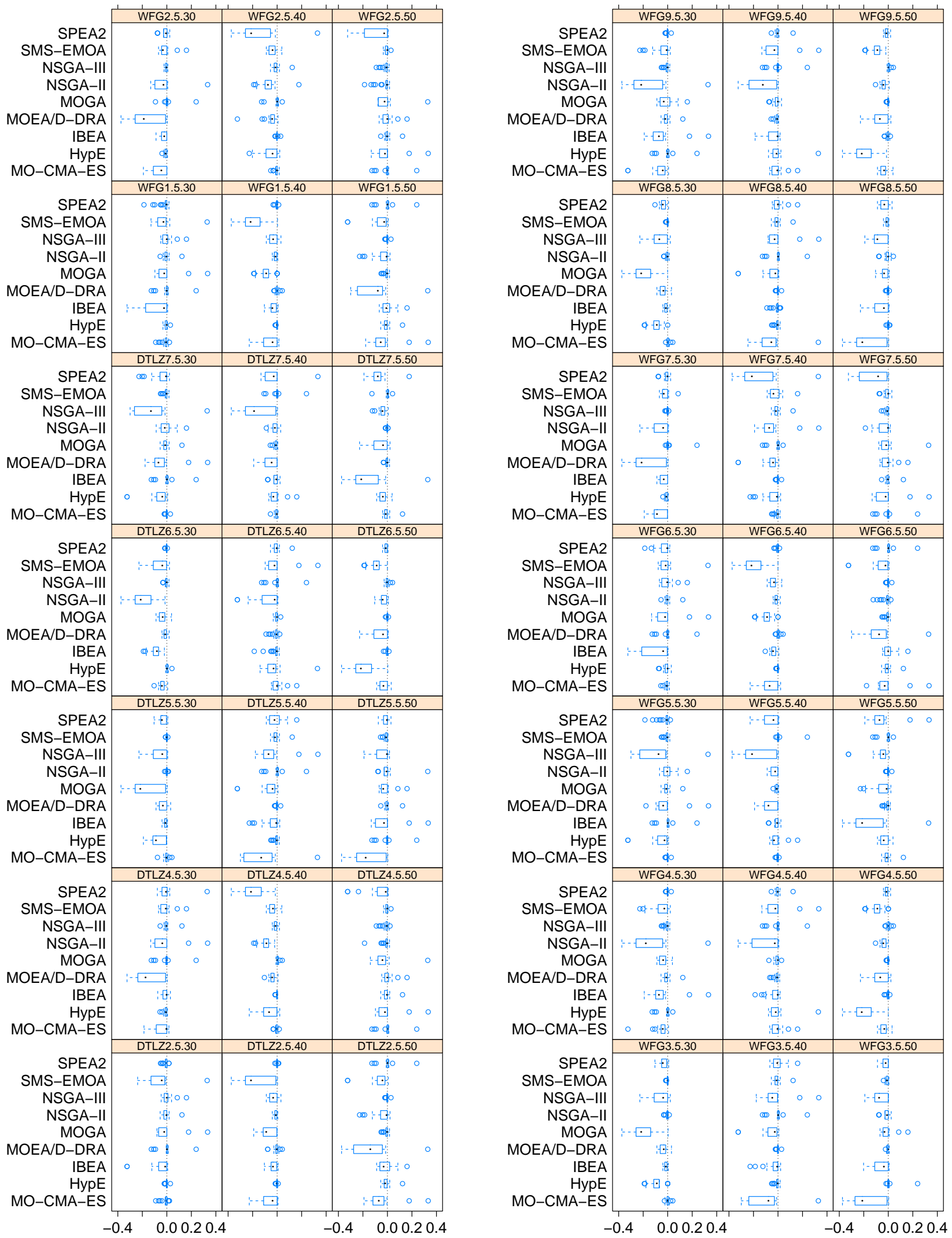

Figure 19: Performance differences according to $I_{H}^{\text {rd }}$ between runs from MOEAs with and without unbounded archiving on the scenario with $M=5$ and $\boldsymbol{F E}_{\max }=2500$ for all problems with $n_{\mathrm{var}} \in\{30,40,50\}$. 

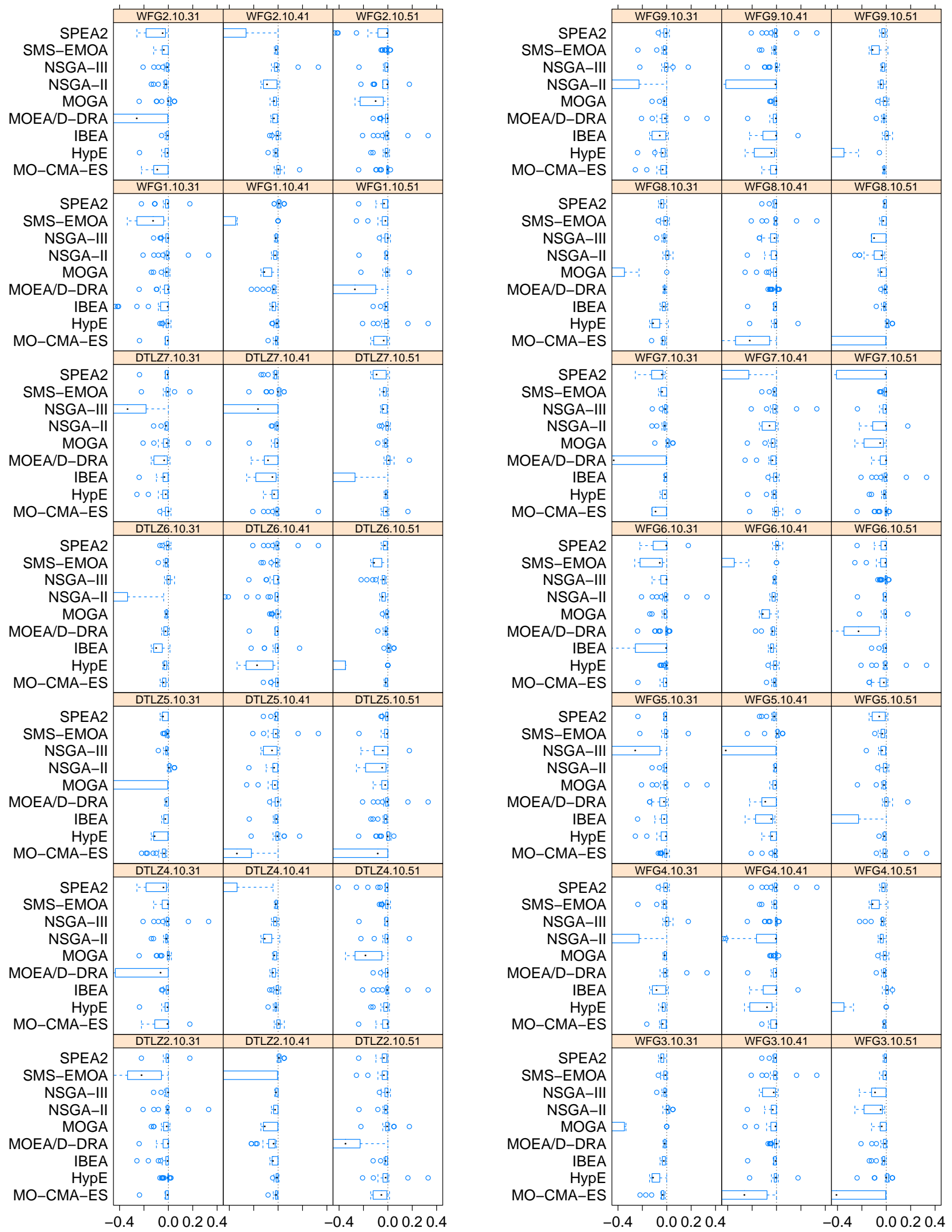

Figure 20: Performance differences according to $I_{H}^{\text {rd }}$ between runs from MOEAs with and without unbounded archiving on the scenario with $M=10$ and $\boldsymbol{F} E_{\max }=2500$ for all problems with $n_{\text {var }} \in\{30,40,50\}$. 

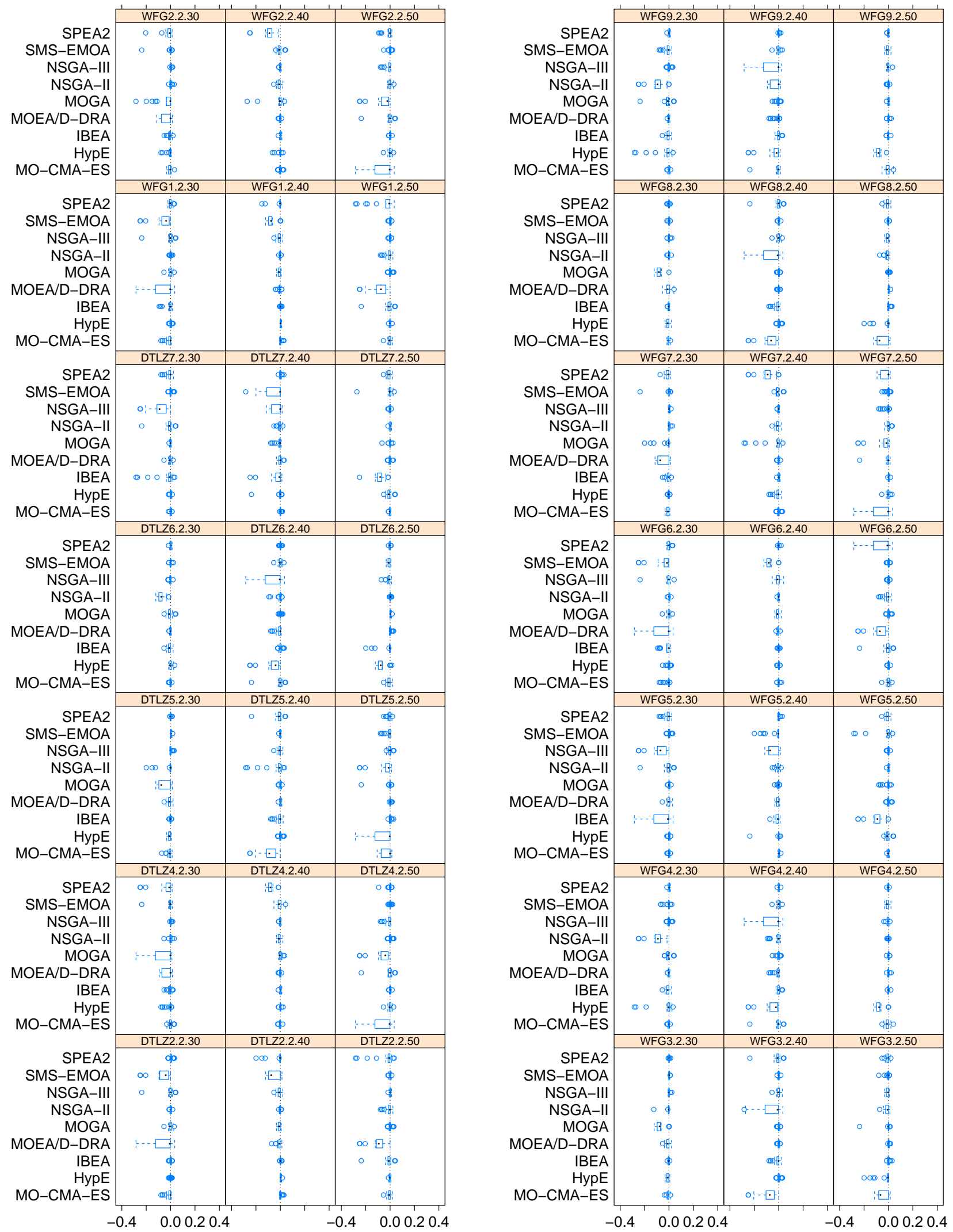

Figure 21: Performance differences according to $I_{H}^{r d}$ between runs from MOEAs with and without unbounded archiving on the scenario with $M=2$ and $\boldsymbol{F} E_{\max }=10000$ for all problems with $n_{\mathrm{var}} \in\{30,40,50\}$. 

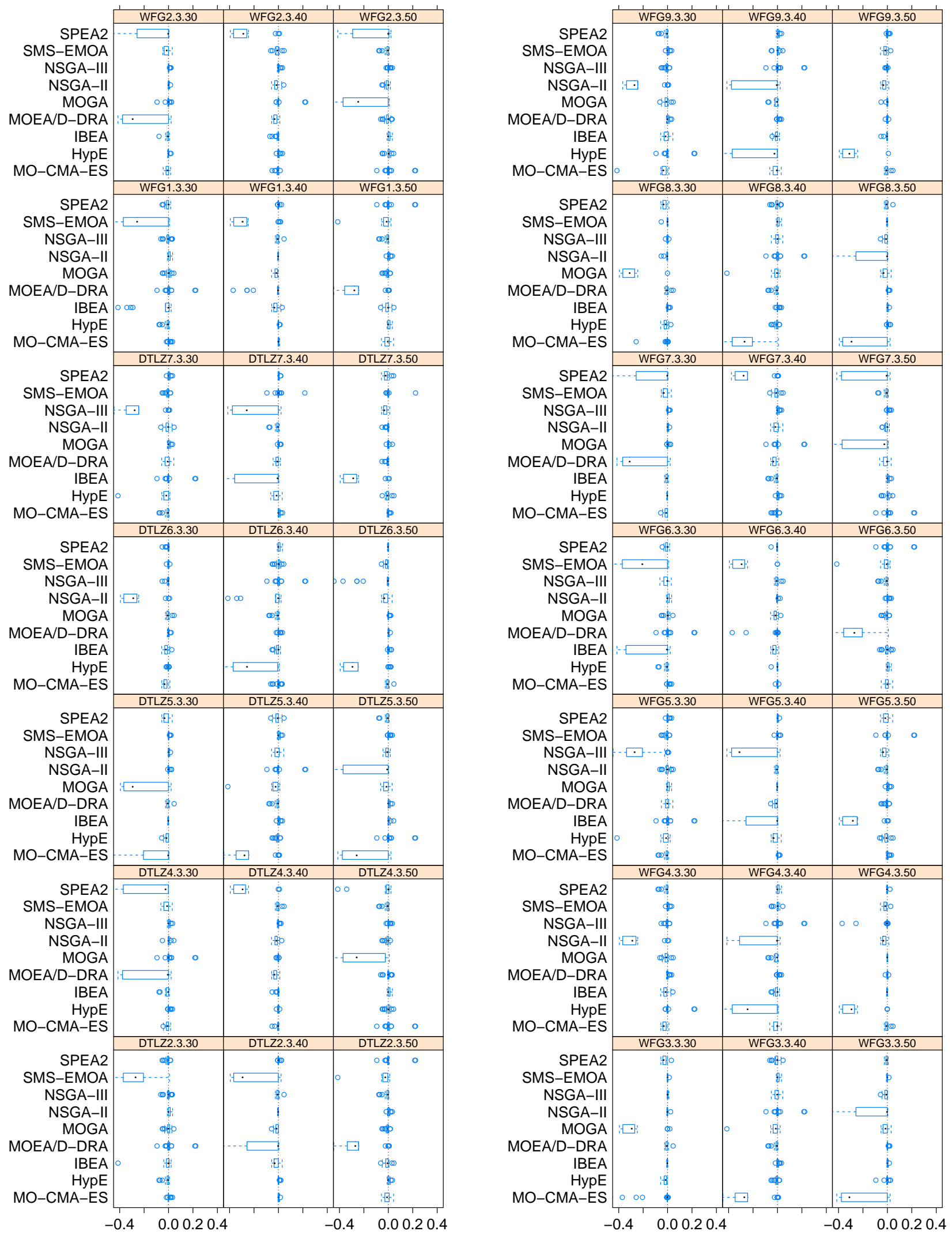

Figure 22: Performance differences according to $I_{H}^{r d}$ between runs from MOEAs with and without unbounded archiving on the scenario with $M=3$ and $\boldsymbol{F} E_{\max }=10000$ for all problems with $n_{\mathrm{var}} \in\{30,40,50\}$. 

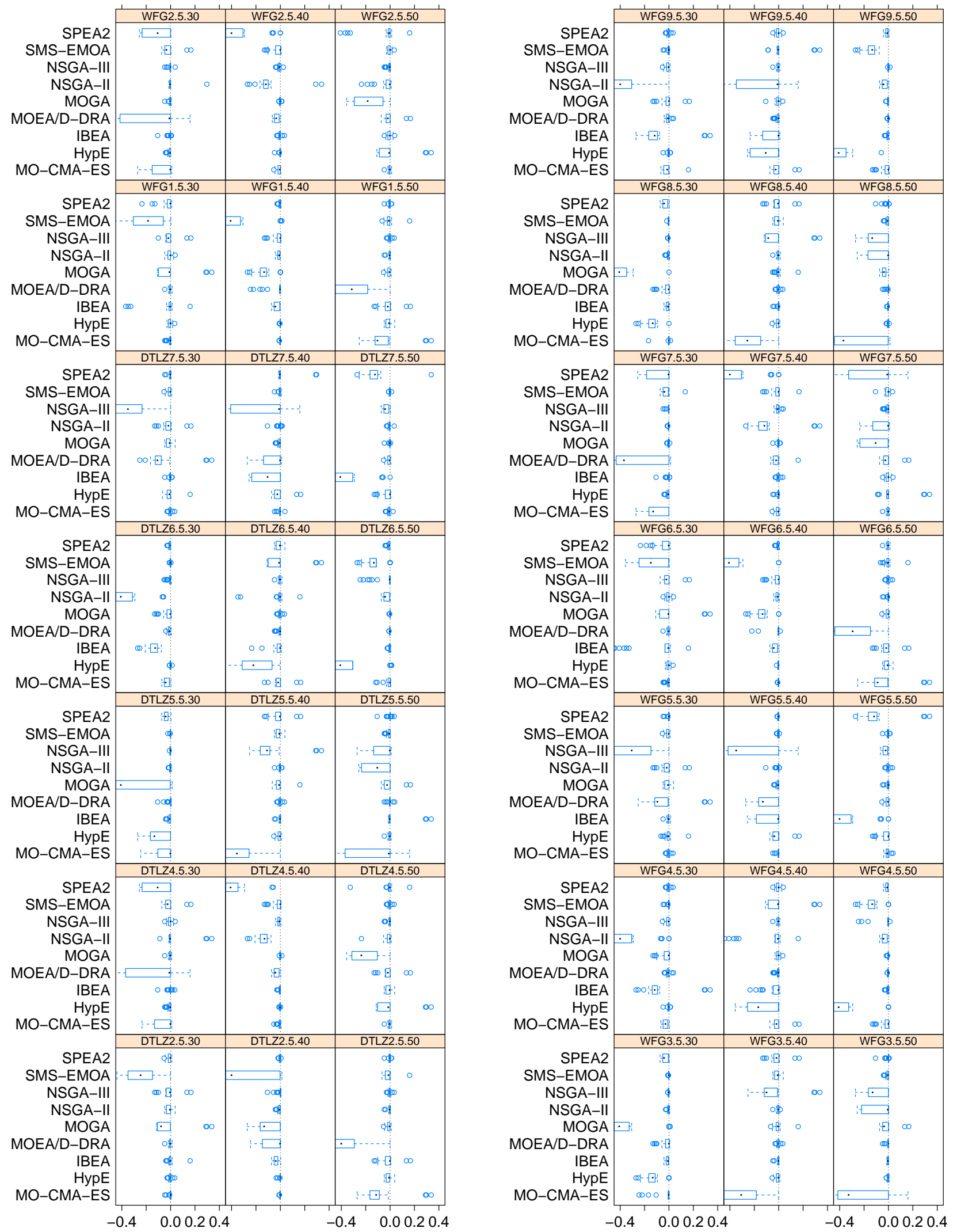

Figure 23: Performance differences according to $I_{H}^{r d}$ between runs from MOEAs with and without unbounded archiving on the scenario with $M=5$ and $\boldsymbol{F} E_{\max }=10000$ for all problems with $n_{\mathrm{var}} \in\{30,40,50\}$. 

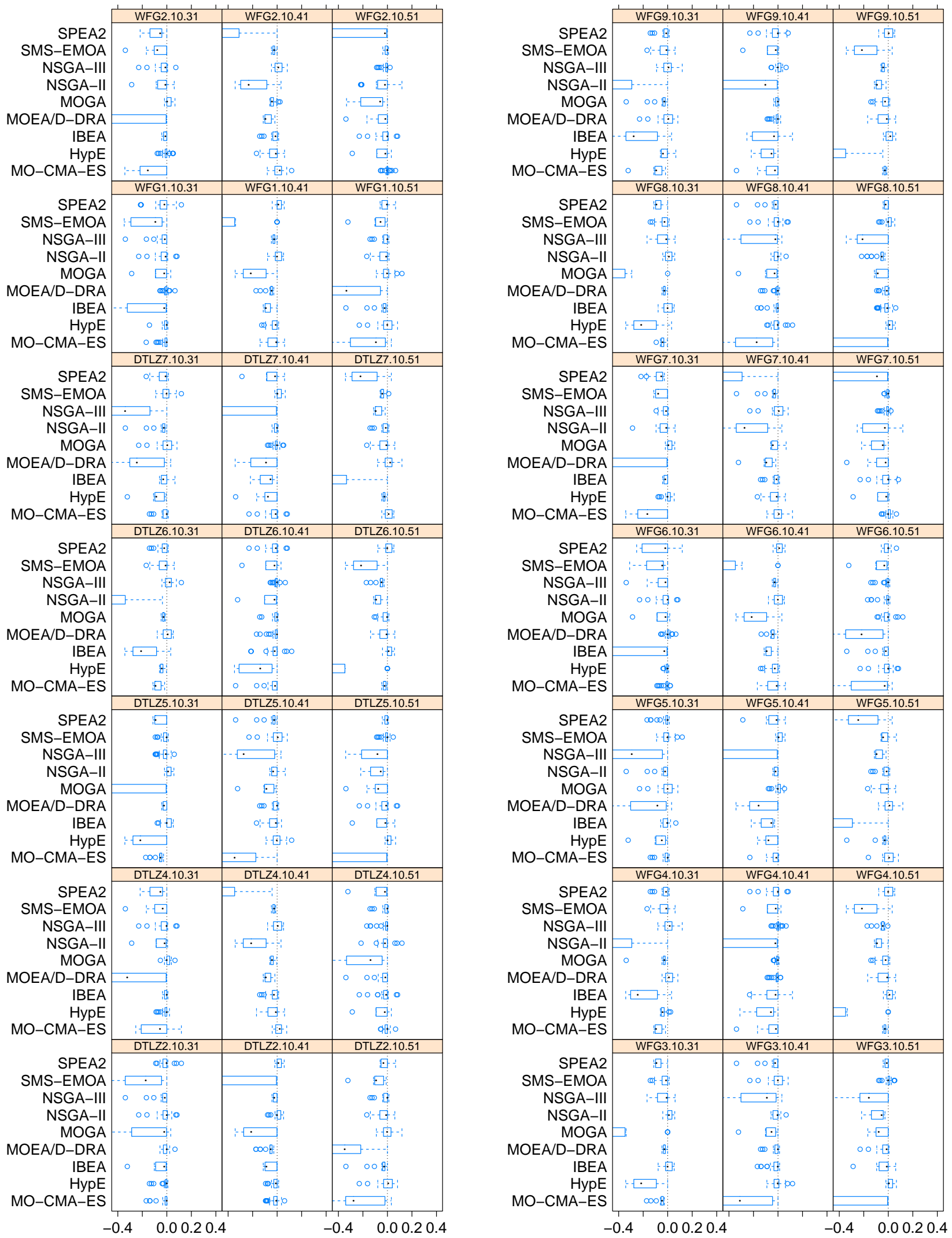

Figure 24: Performance differences according to $I_{H}^{\text {rd }}$ between runs from MOEAs with and without unbounded archiving on the scenario with $M=10$ and $\boldsymbol{F} E_{\max }=10000$ for all problems with $n_{\mathrm{var}} \in\{30,40,50\}$. 

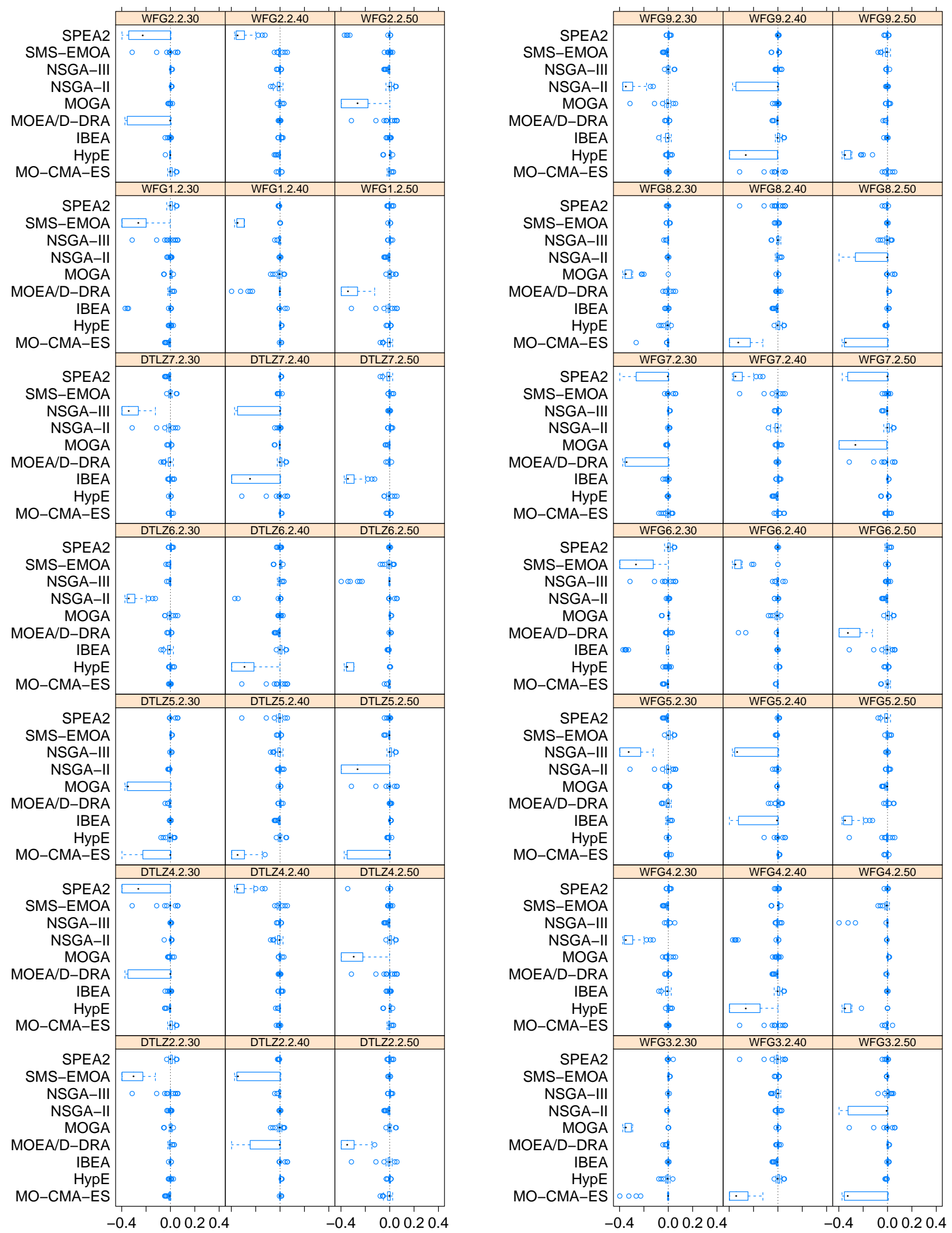

Figure 25: Performance differences according to $I_{H}^{\text {rd }}$ between runs from MOEAs with and without unbounded archiving on the scenario with $M=2$ and $\boldsymbol{F} E_{\max }=40000$ for all problems with $n_{\mathrm{var}} \in\{30,40,50\}$. 

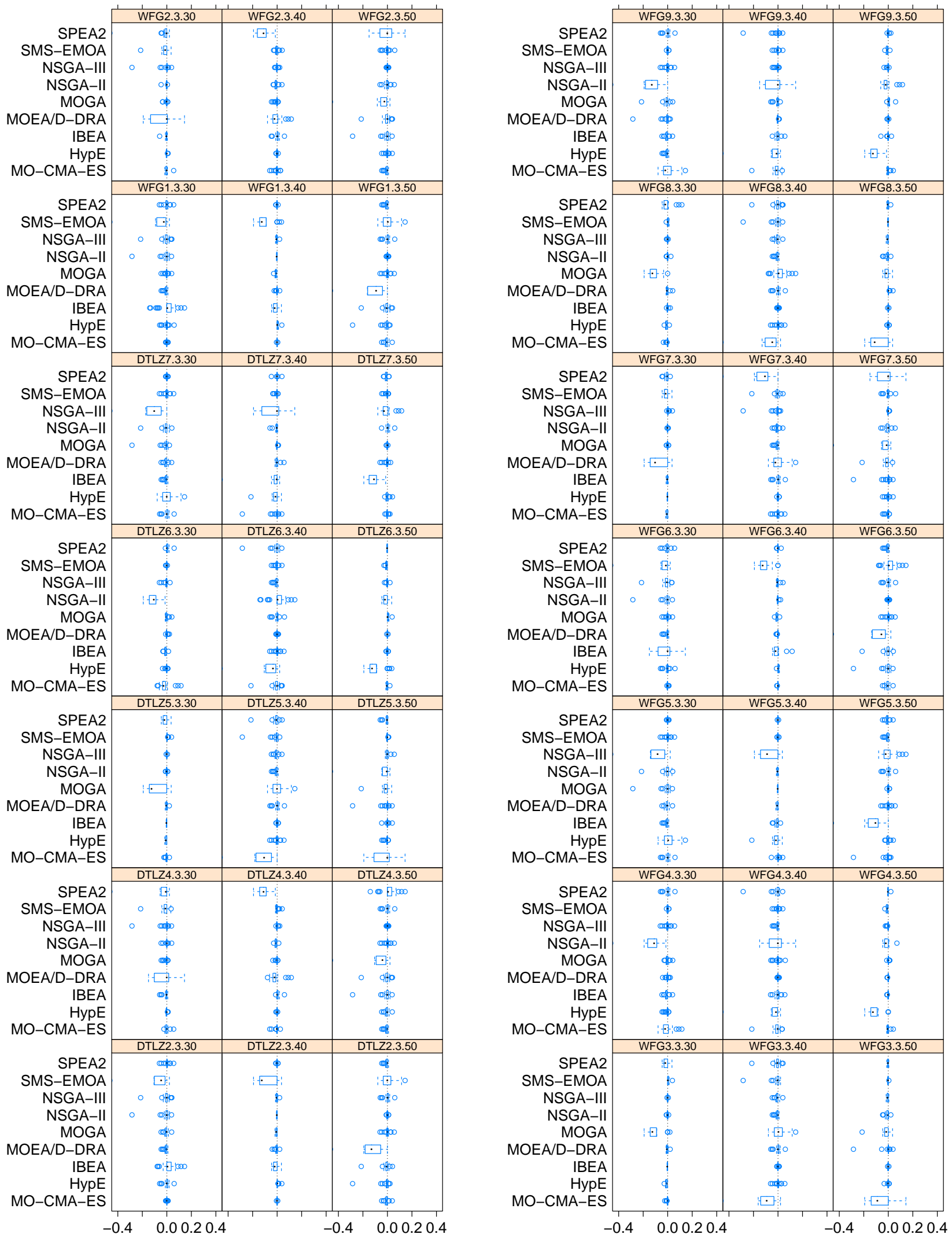

Figure 26: Performance differences according to $I_{H}^{\text {rd }}$ between runs from MOEAs with and without unbounded archiving on the scenario with $M=3$ and $\boldsymbol{F} E_{\max }=40000$ for all problems with $n_{\mathrm{var}} \in\{30,40,50\}$. 

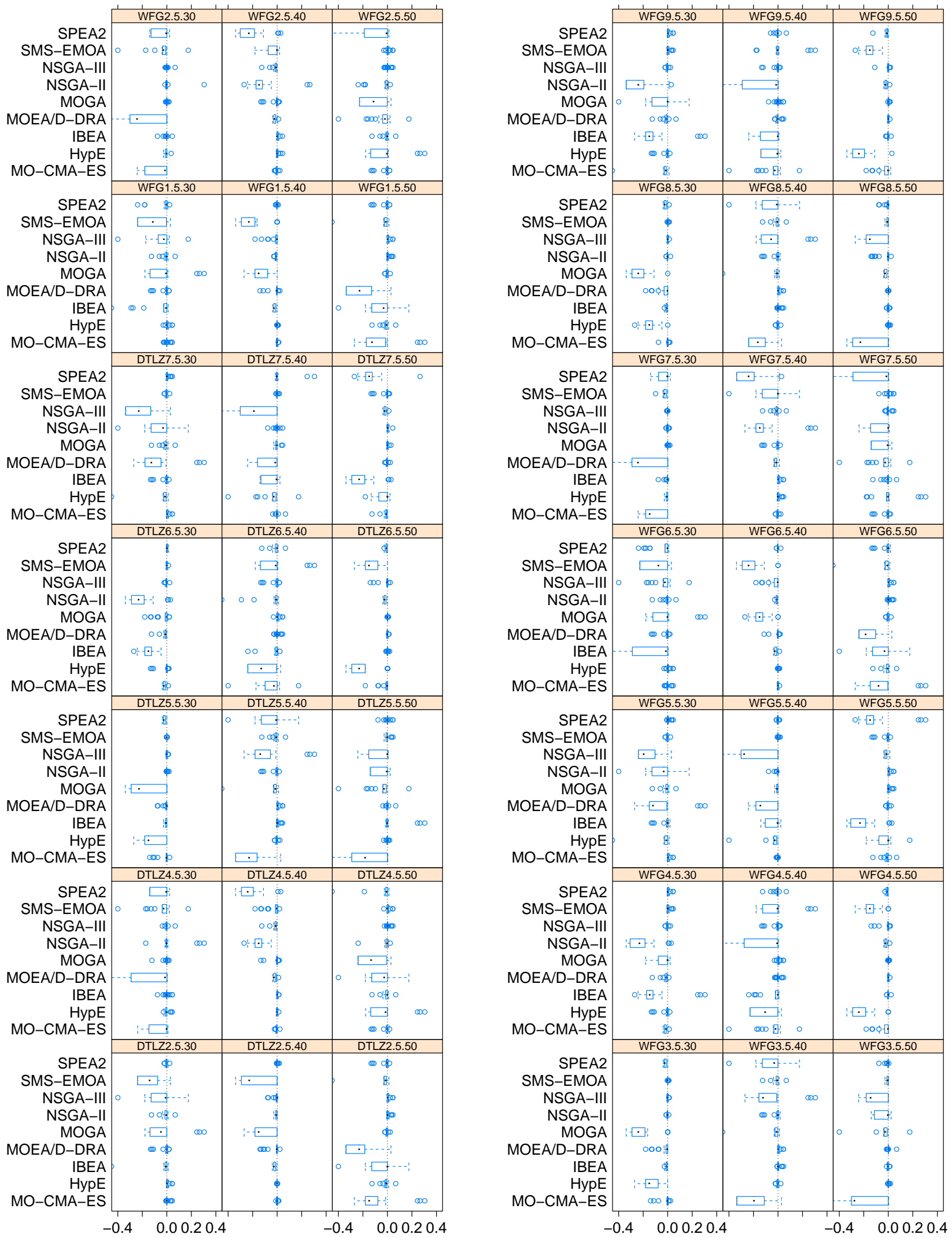

Figure 27: Performance differences according to $I_{H}^{\text {rd }}$ between runs from MOEAs with and without unbounded archiving on the scenario with $M=5$ and $\boldsymbol{F} E_{\max }=40000$ for all problems with $n_{\mathrm{var}} \in\{30,40,50\}$. 

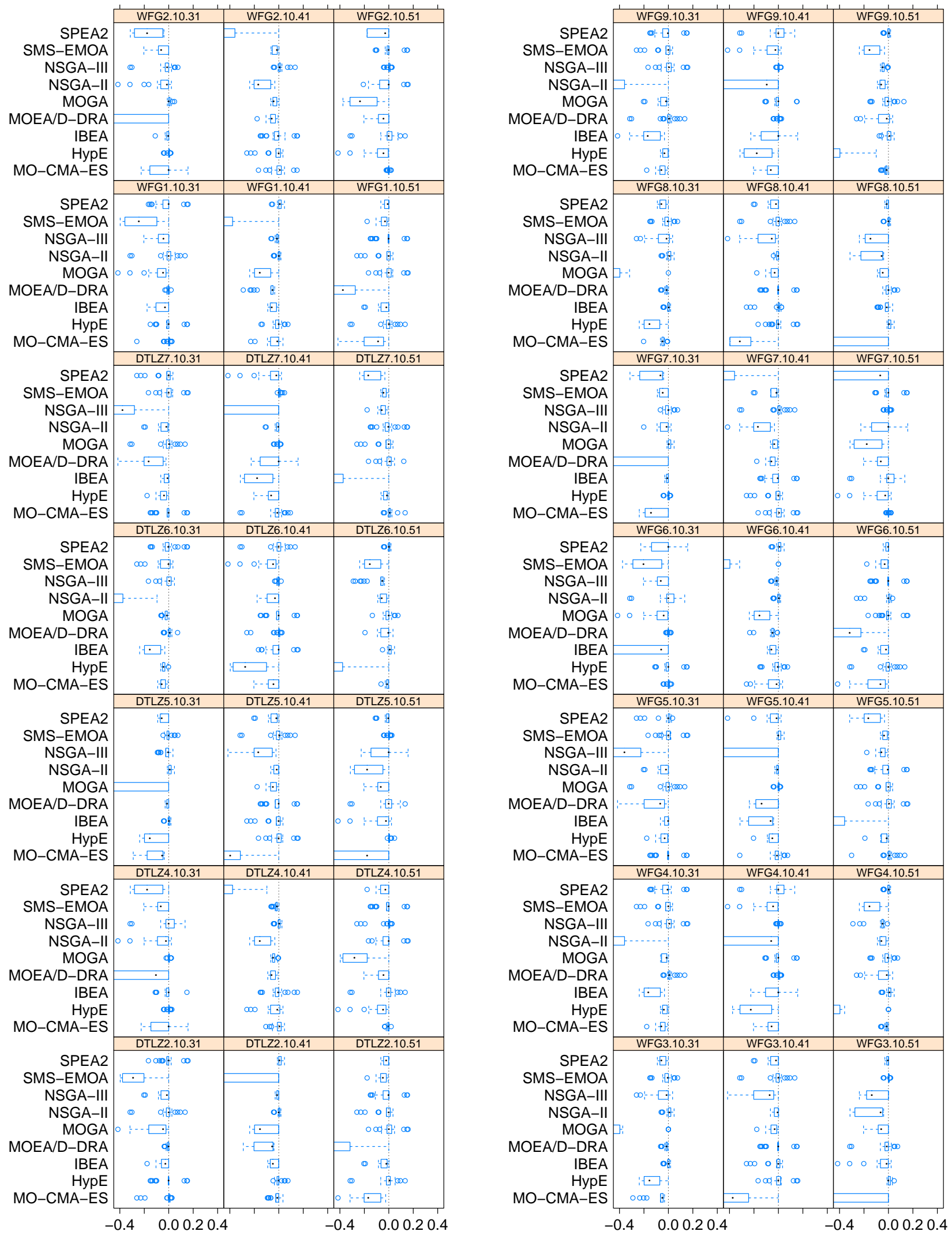

Figure 28: Performance differences according to $I_{H}^{\text {rd }}$ between runs from MOEAs with and without unbounded archiving on the scenario with $M=10$ and $\boldsymbol{F} E_{\max }=40000$ for all problems with $n_{\mathrm{var}} \in\{30,40,50\}$. 

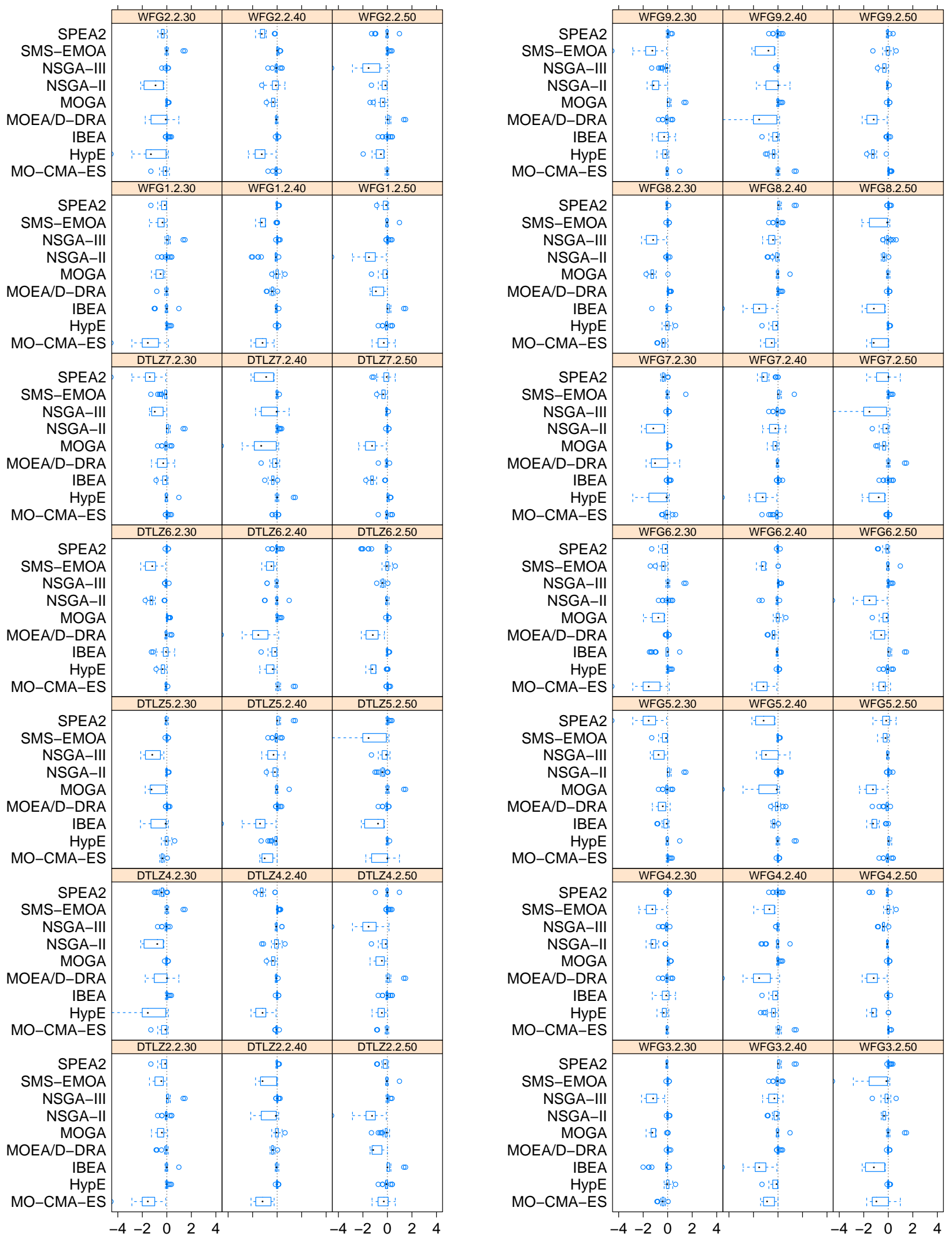

Figure 29: Performance differences according to $I_{\epsilon+}$ between runs from MOEAs with and without unbounded archiving on the scenario with $M=2$ and $\boldsymbol{F E}_{\max }=2500$ for all problems with $n_{\mathbf{v a r}} \in\{30,40,50\}$. 

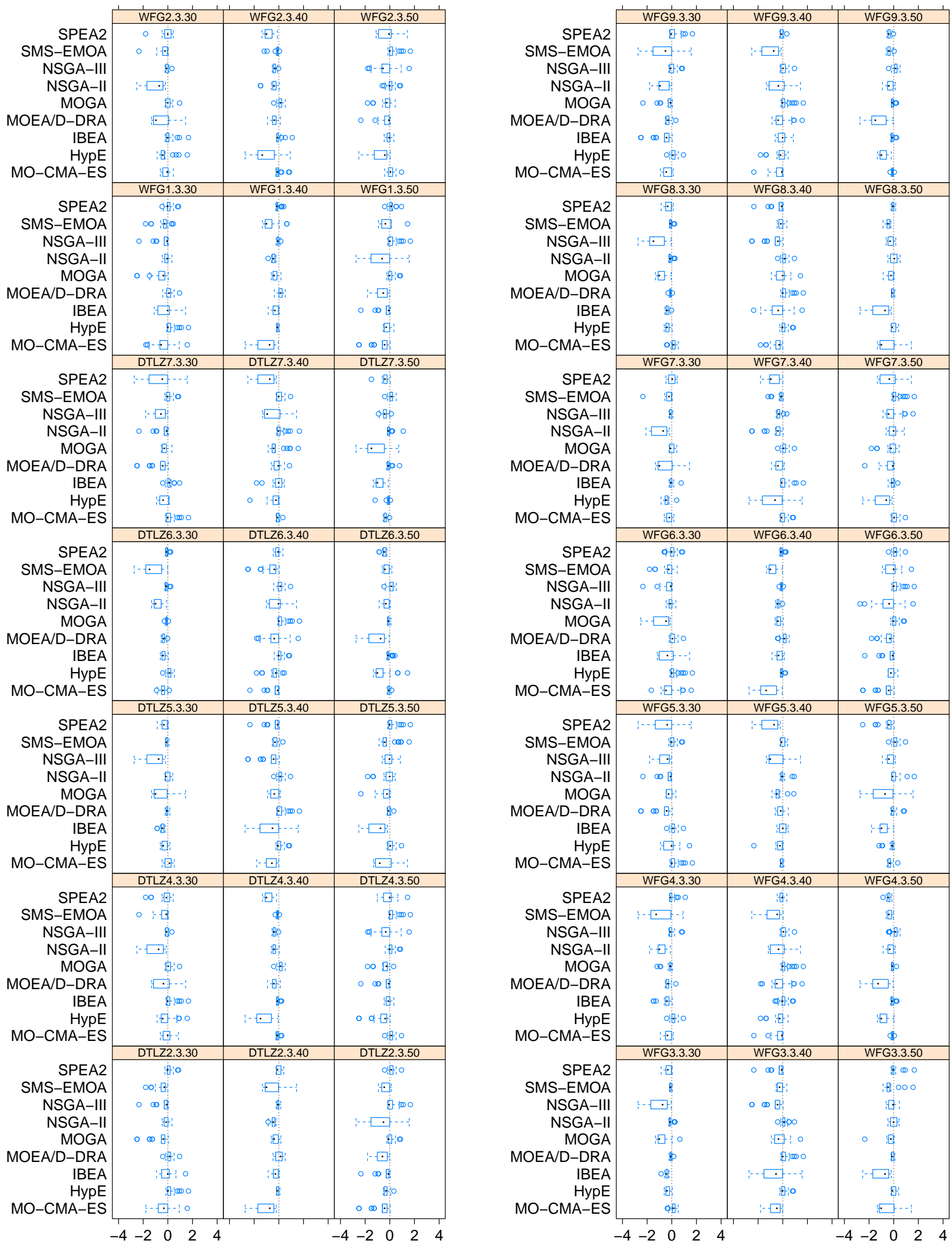

Figure 30: Performance differences according to $I_{\epsilon+}$ between runs from MOEAs with and without unbounded archiving on the scenario with $M=3$ and $\boldsymbol{F} E_{\max }=2500$ for all problems with $n_{\text {var }} \in\{30,40,50\}$. 

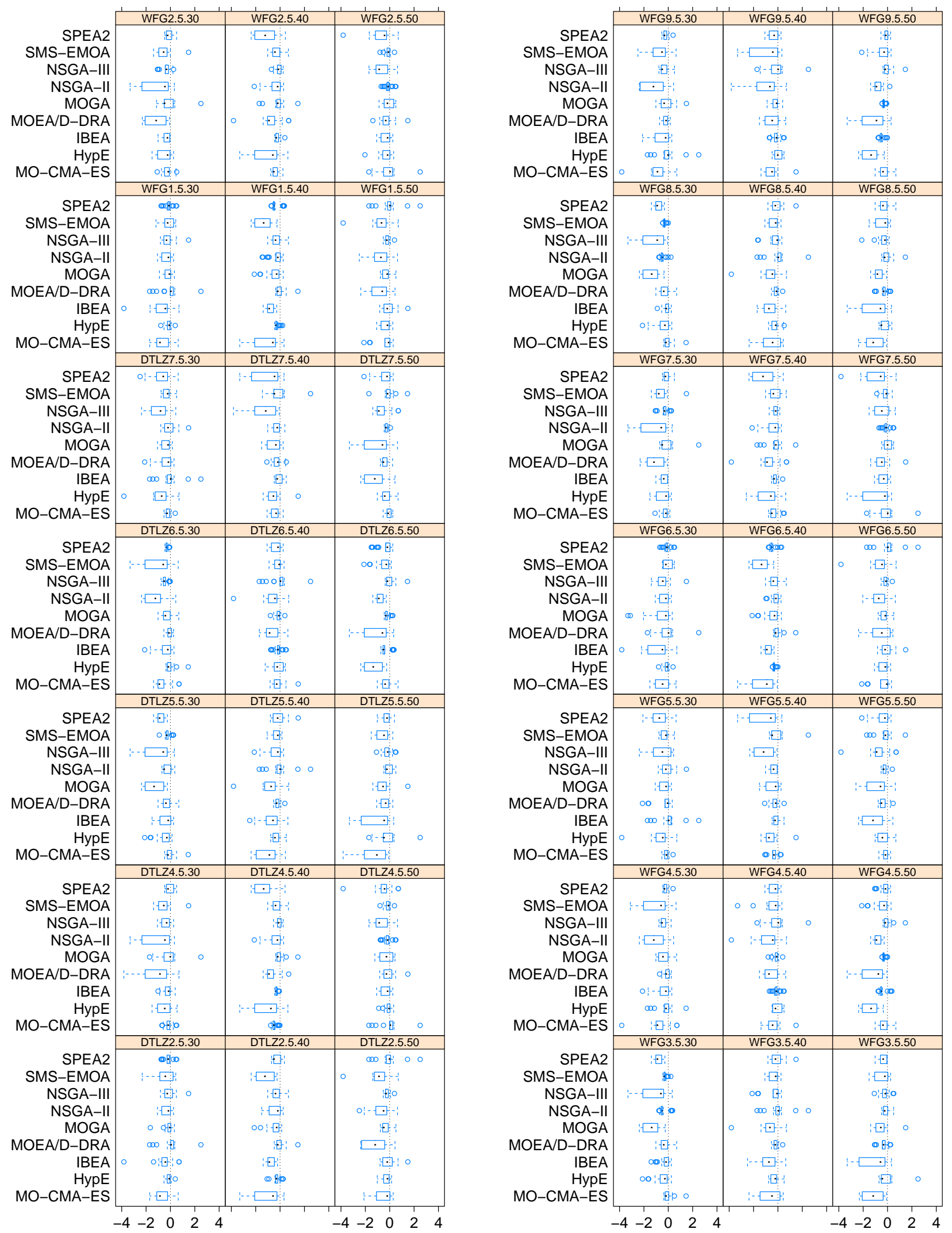

Figure 31: Performance differences according to $I_{\epsilon+}$ between runs from MOEAs with and without unbounded archiving on the scenario with $M=5$ and $\boldsymbol{F} E_{\max }=2500$ for all problems with $n_{\text {var }} \in\{30,40,50\}$. 

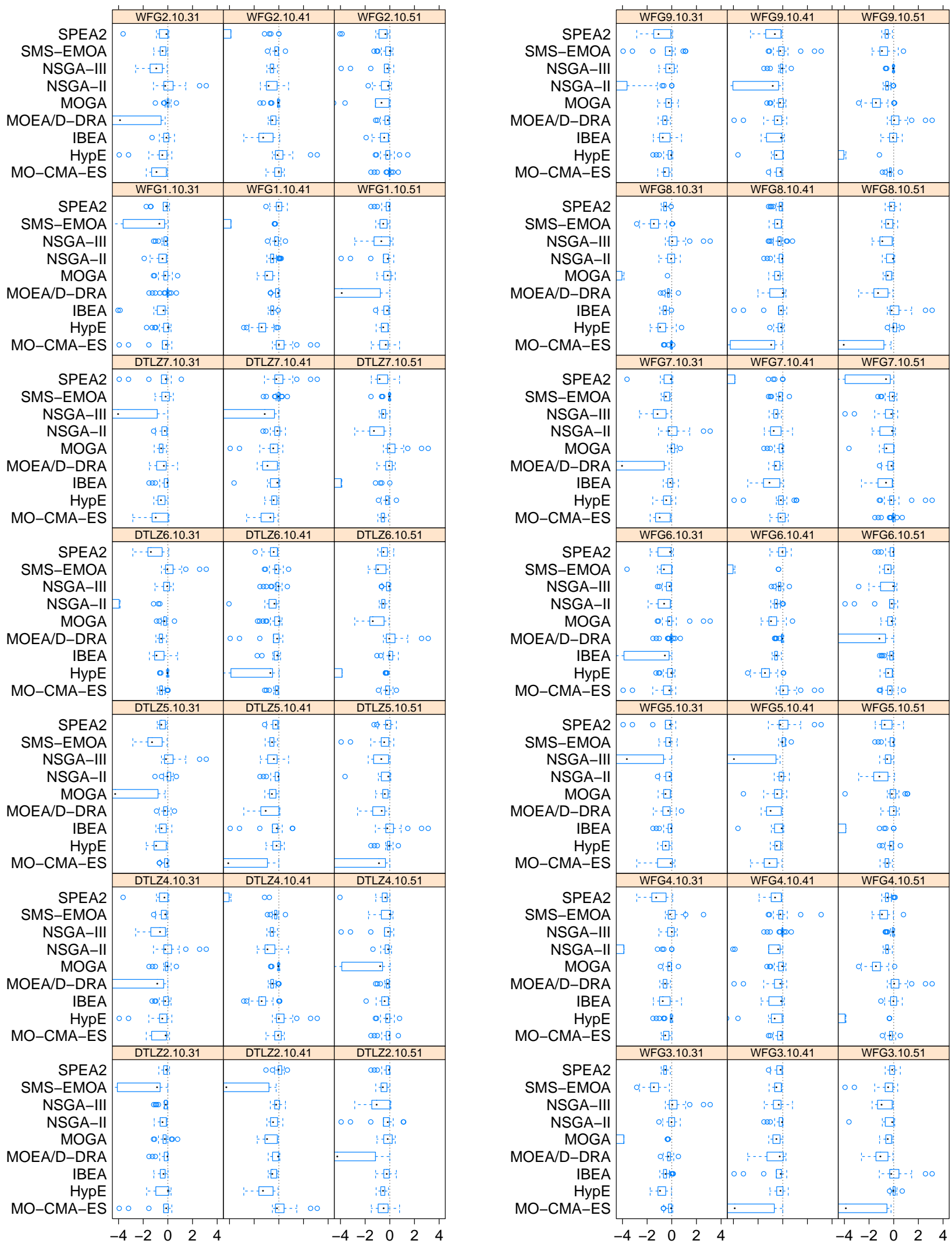

Figure 32: Performance differences according to $I_{\epsilon+}$ between runs from MOEAs with and without unbounded archiving on the scenario with $M=10$ and $\boldsymbol{F} E_{\max }=2500$ for all problems with $n_{\text {var }} \in\{30,40,50\}$. 

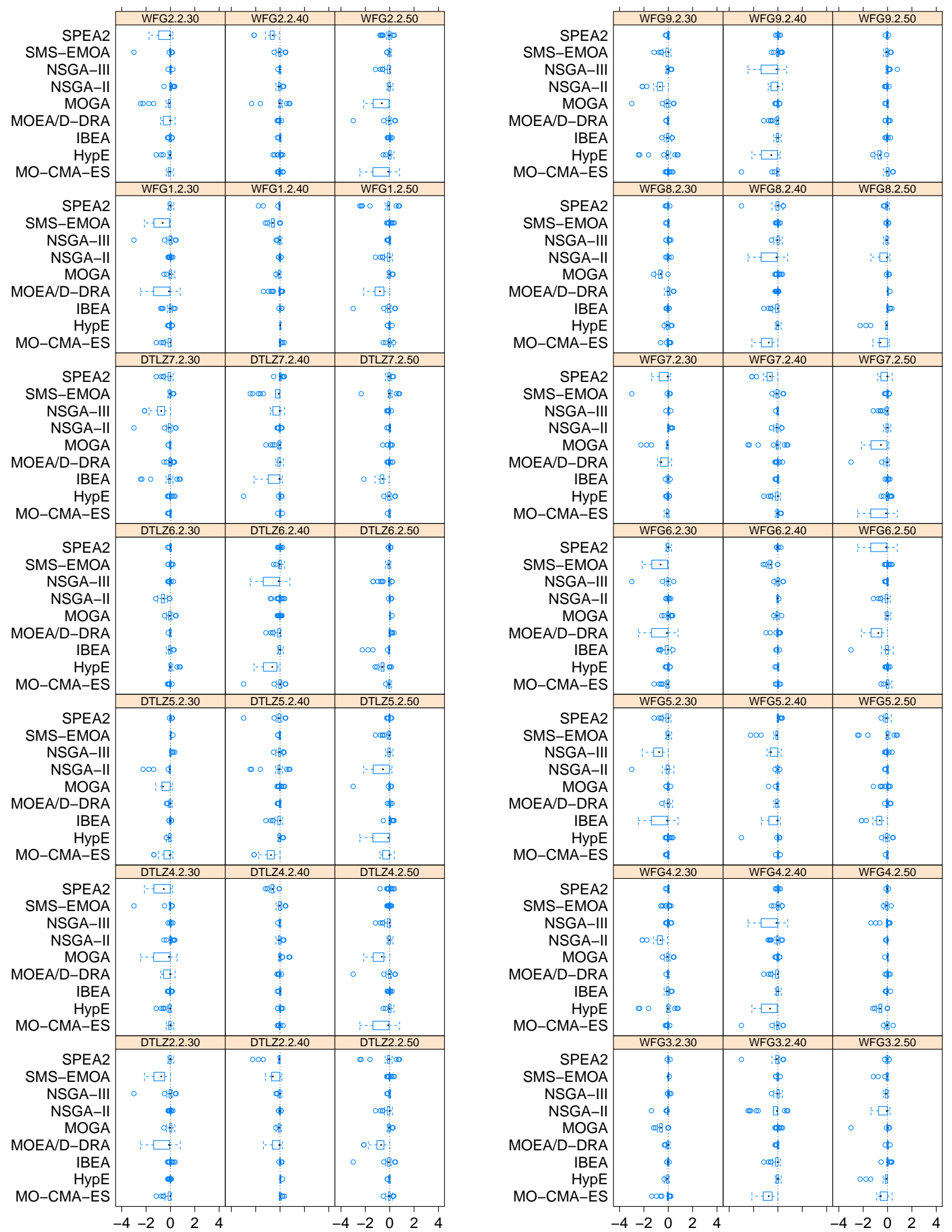

Figure 33: Performance differences according to $I_{\epsilon+}$ between runs from MOEAs with and without unbounded archiving on the scenario with $M=2$ and $\boldsymbol{F} E_{\max }=10000$ for all problems with $n_{\text {var }} \in\{30,40,50\}$. 

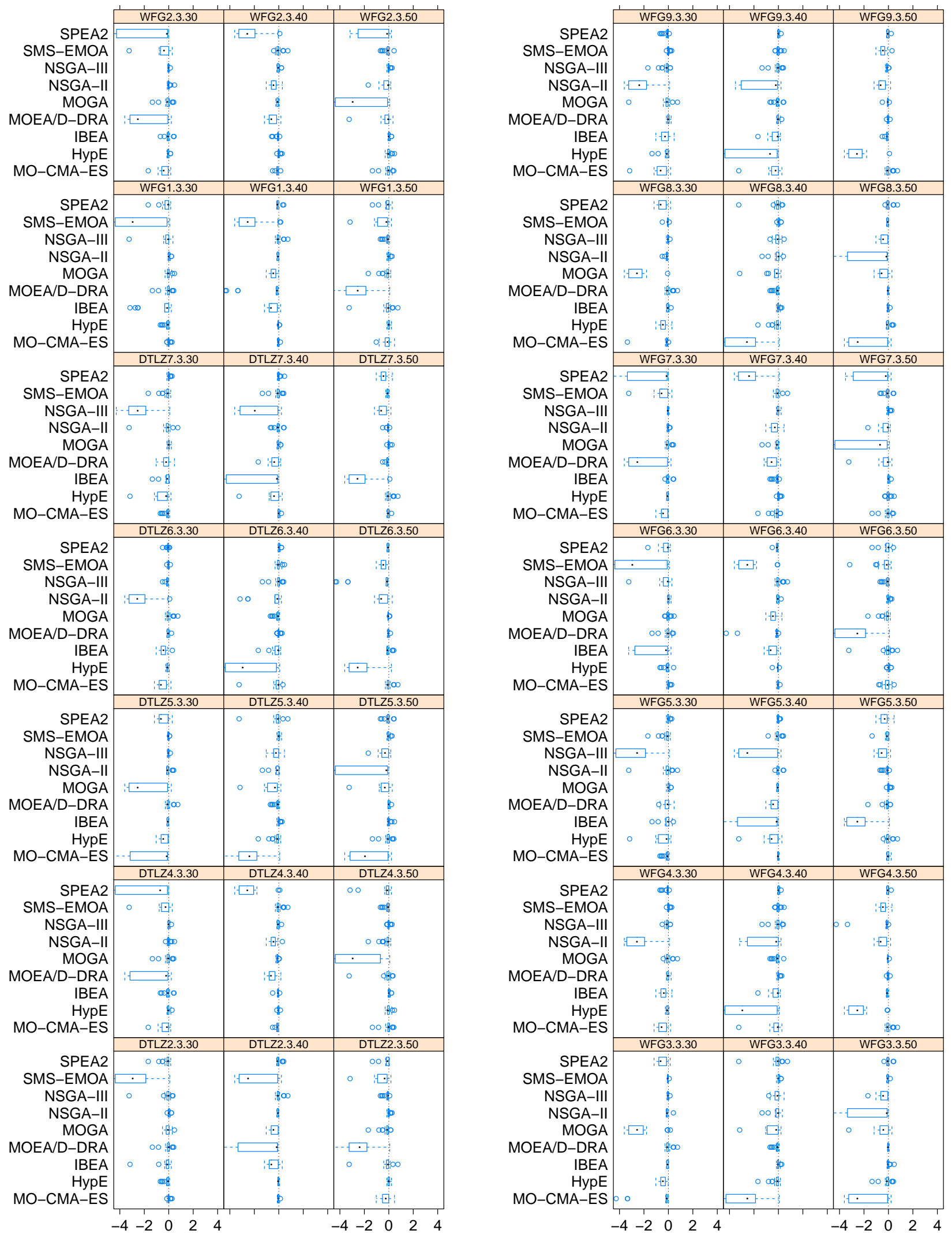

Figure 34: Performance differences according to $I_{\epsilon+}$ between runs from MOEAs with and without unbounded archiving on the scenario with $M=3$ and $\boldsymbol{F} E_{\max }=10000$ for all problems with $n_{\text {var }} \in\{30,40,50\}$. 

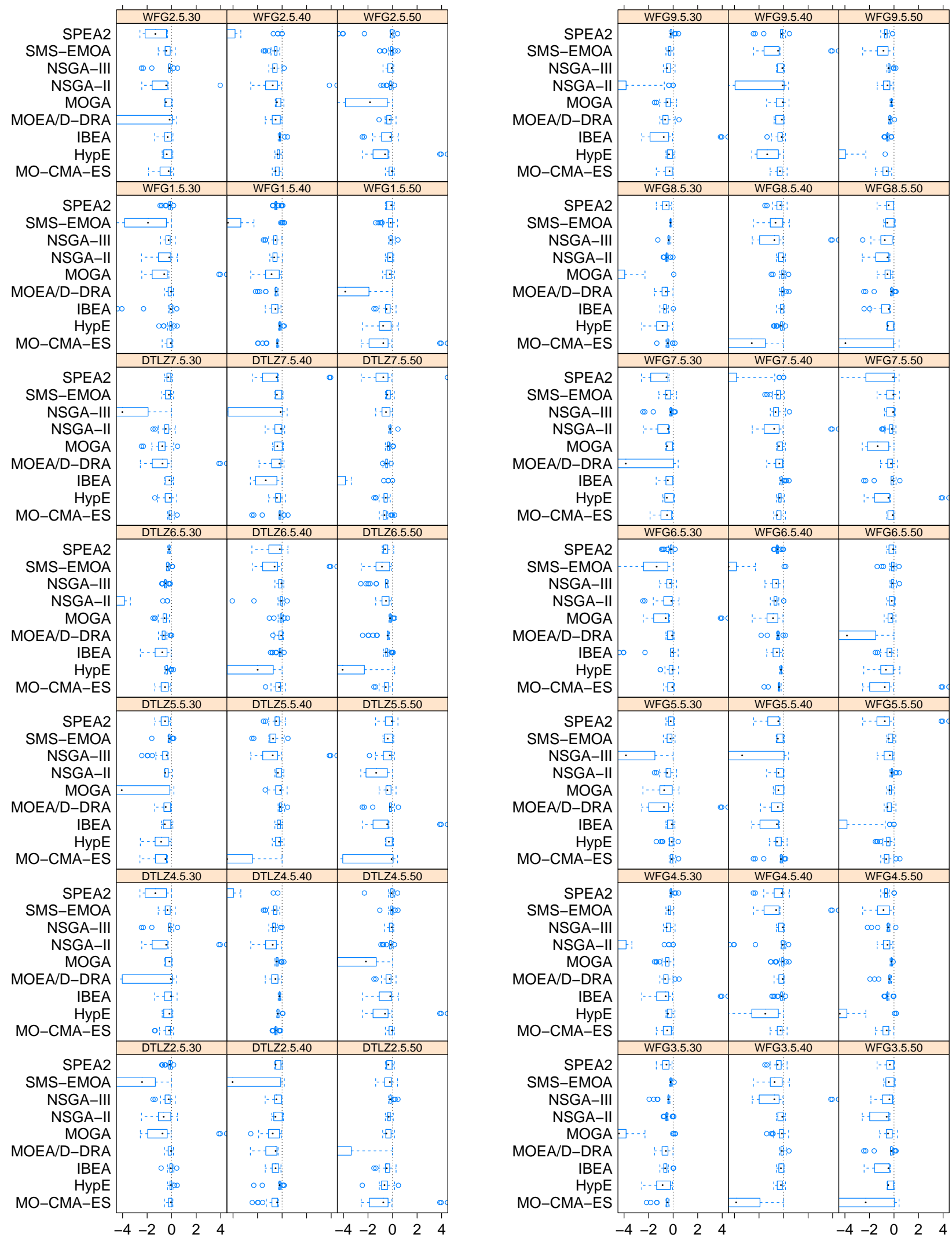

Figure 35: Performance differences according to $I_{\epsilon+}$ between runs from MOEAs with and without unbounded archiving on the scenario with $M=5$ and $\boldsymbol{F} E_{\max }=10000$ for all problems with $n_{\text {var }} \in\{30,40,50\}$. 

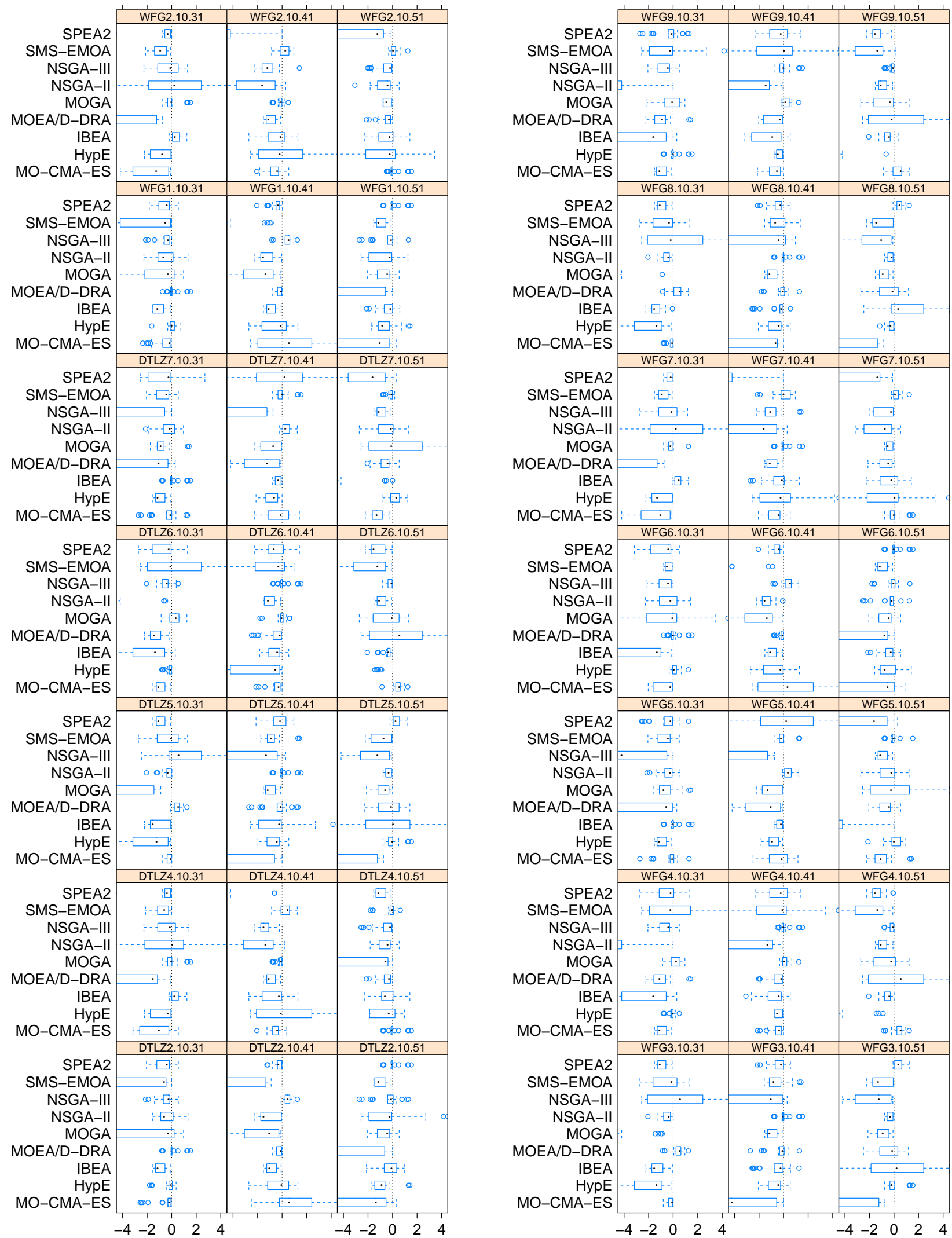

Figure 36: Performance differences according to $I_{\epsilon+}$ between runs from MOEAs with and without unbounded archiving on the scenario with $M=10$ and $\boldsymbol{F} \boldsymbol{E}_{\max }=10000$ for all problems with $n_{\mathrm{var}} \in\{30,40,50\}$. 

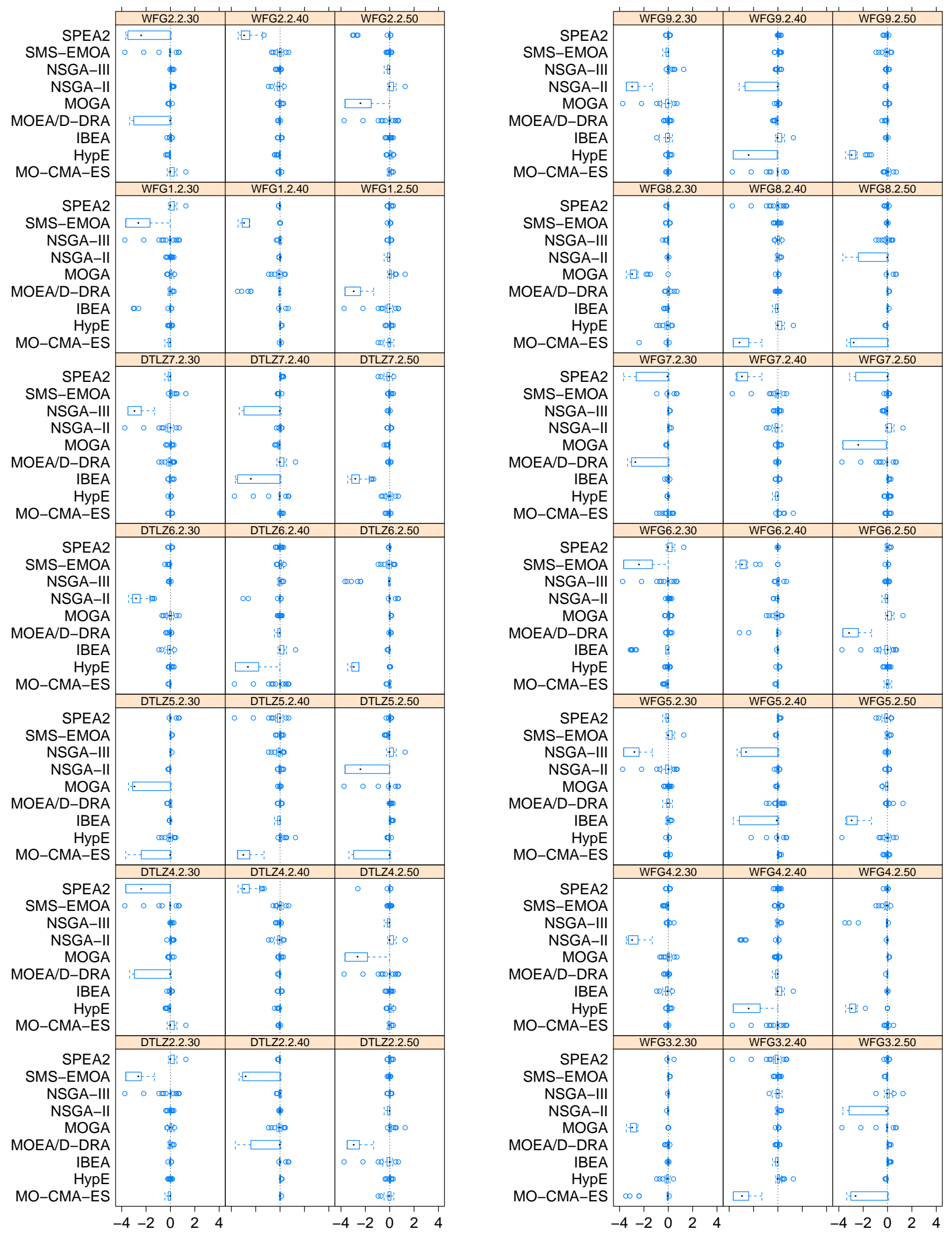

Figure 37: Performance differences according to $I_{\epsilon+}$ between runs from MOEAs with and without unbounded archiving on the scenario with $M=2$ and $\boldsymbol{F} E_{\max }=40000$ for all problems with $n_{\text {var }} \in\{30,40,50\}$. 

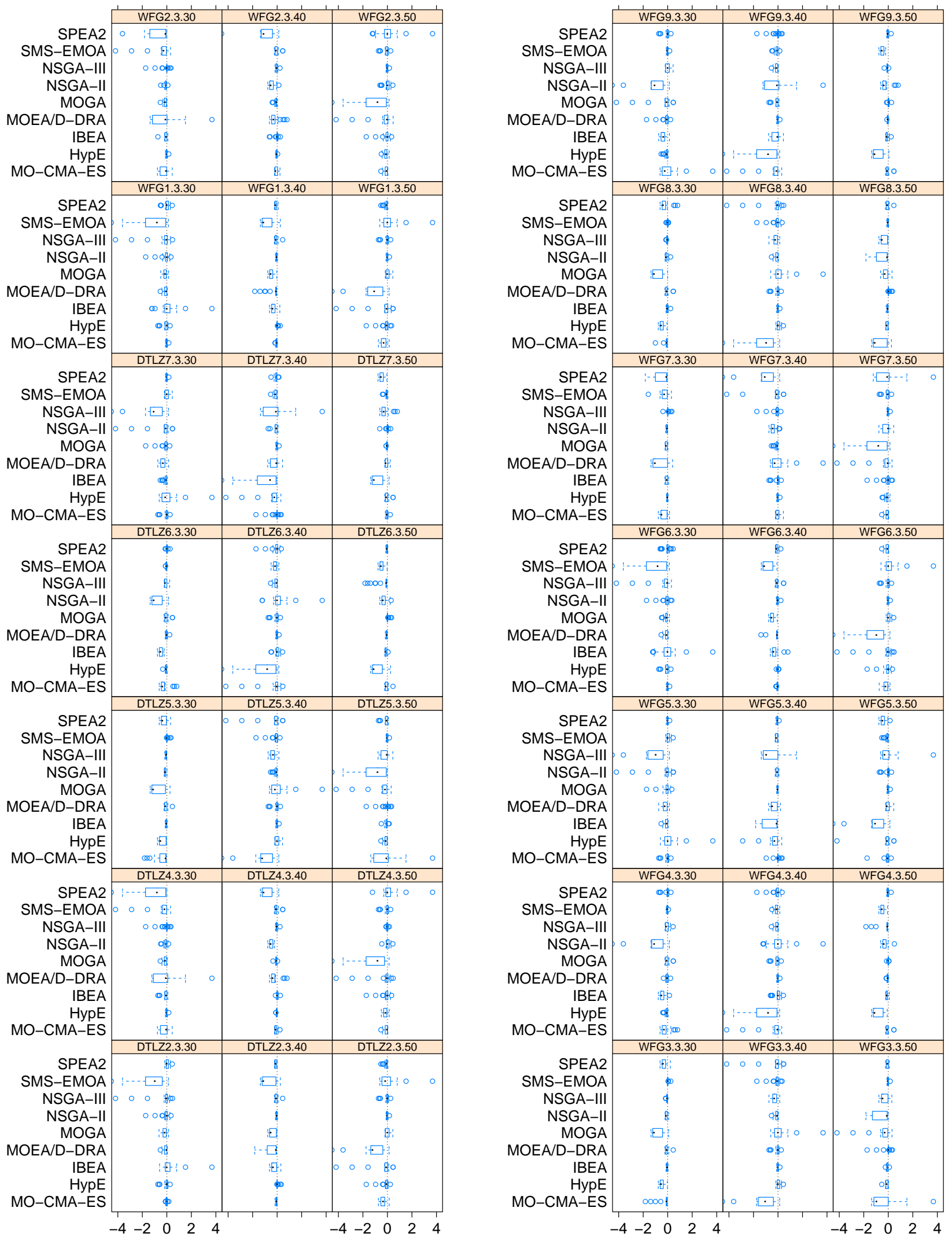

Figure 38: Performance differences according to $I_{\epsilon+}$ between runs from MOEAs with and without unbounded archiving on the scenario with $M=3$ and $\boldsymbol{F} E_{\max }=40000$ for all problems with $n_{\text {var }} \in\{30,40,50\}$. 

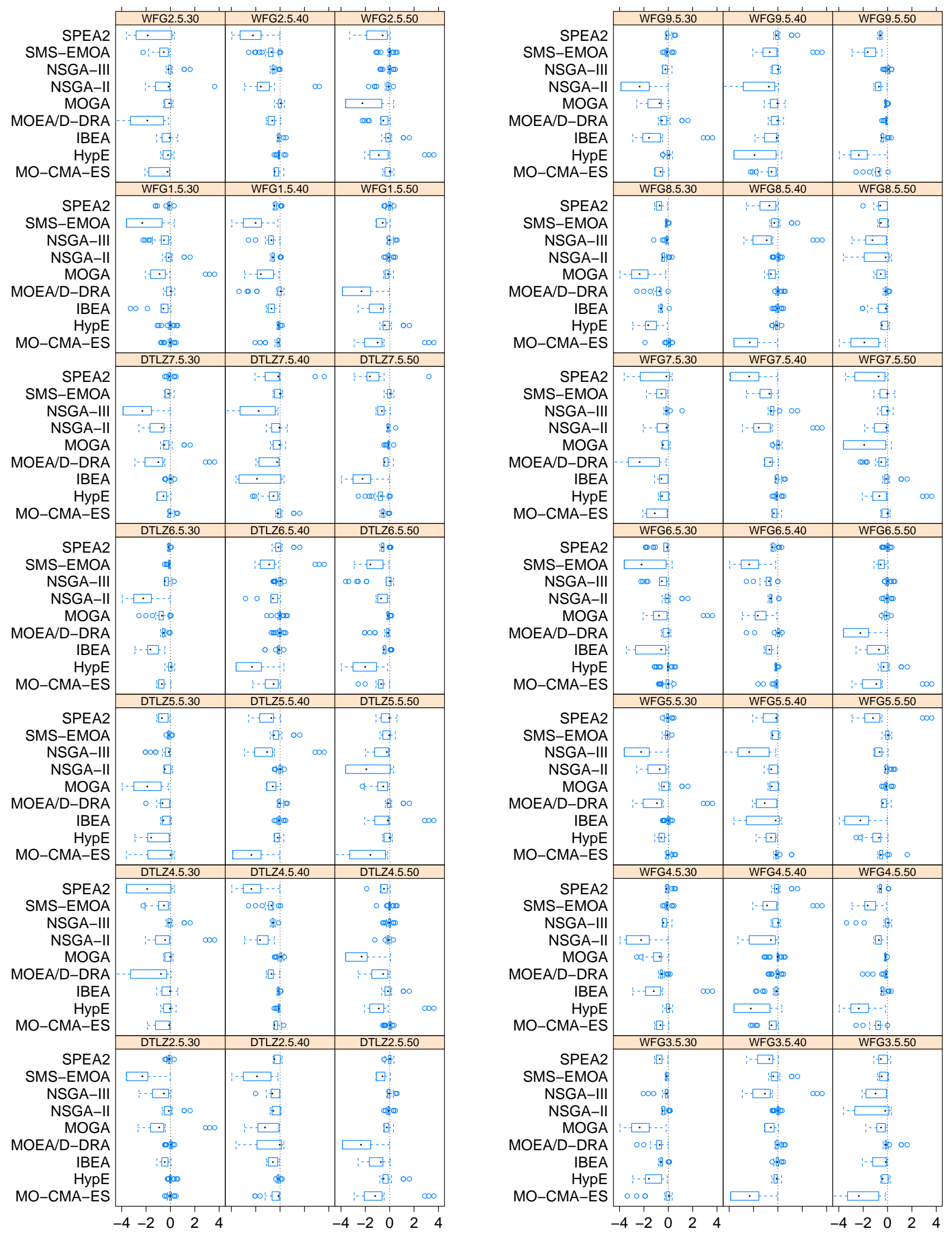

Figure 39: Performance differences according to $I_{\epsilon+}$ between runs from MOEAs with and without unbounded archiving on the scenario with $M=5$ and $\boldsymbol{F} E_{\max }=40000$ for all problems with $n_{\text {var }} \in\{30,40,50\}$. 

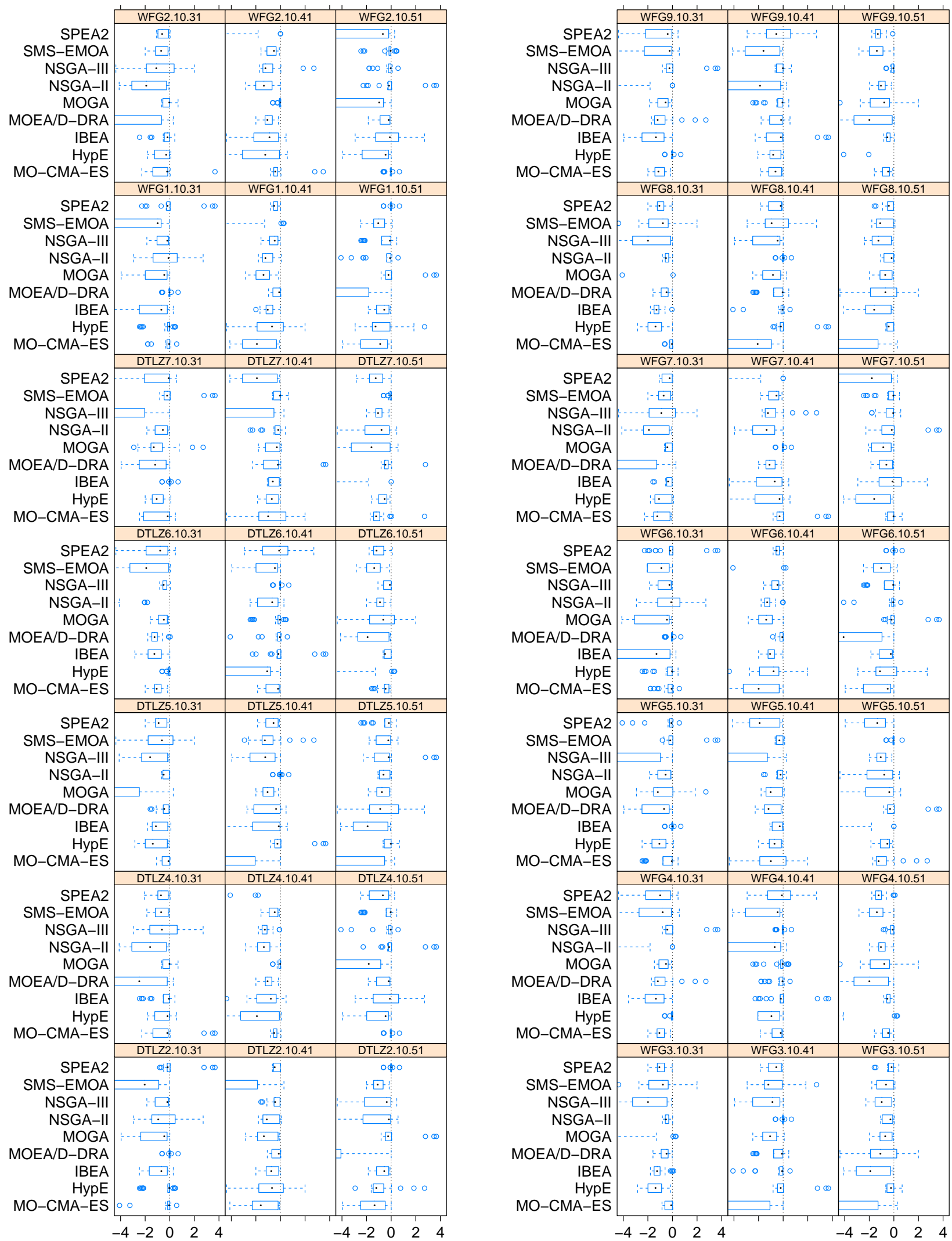

Figure 40: Performance differences according to $I_{\epsilon+}$ between runs from MOEAs with and without unbounded archiving on the scenario with $M=10$ and $\boldsymbol{F} \boldsymbol{E}_{\max }=40000$ for all problems with $n_{\mathrm{var}} \in\{30,40,50\}$. 

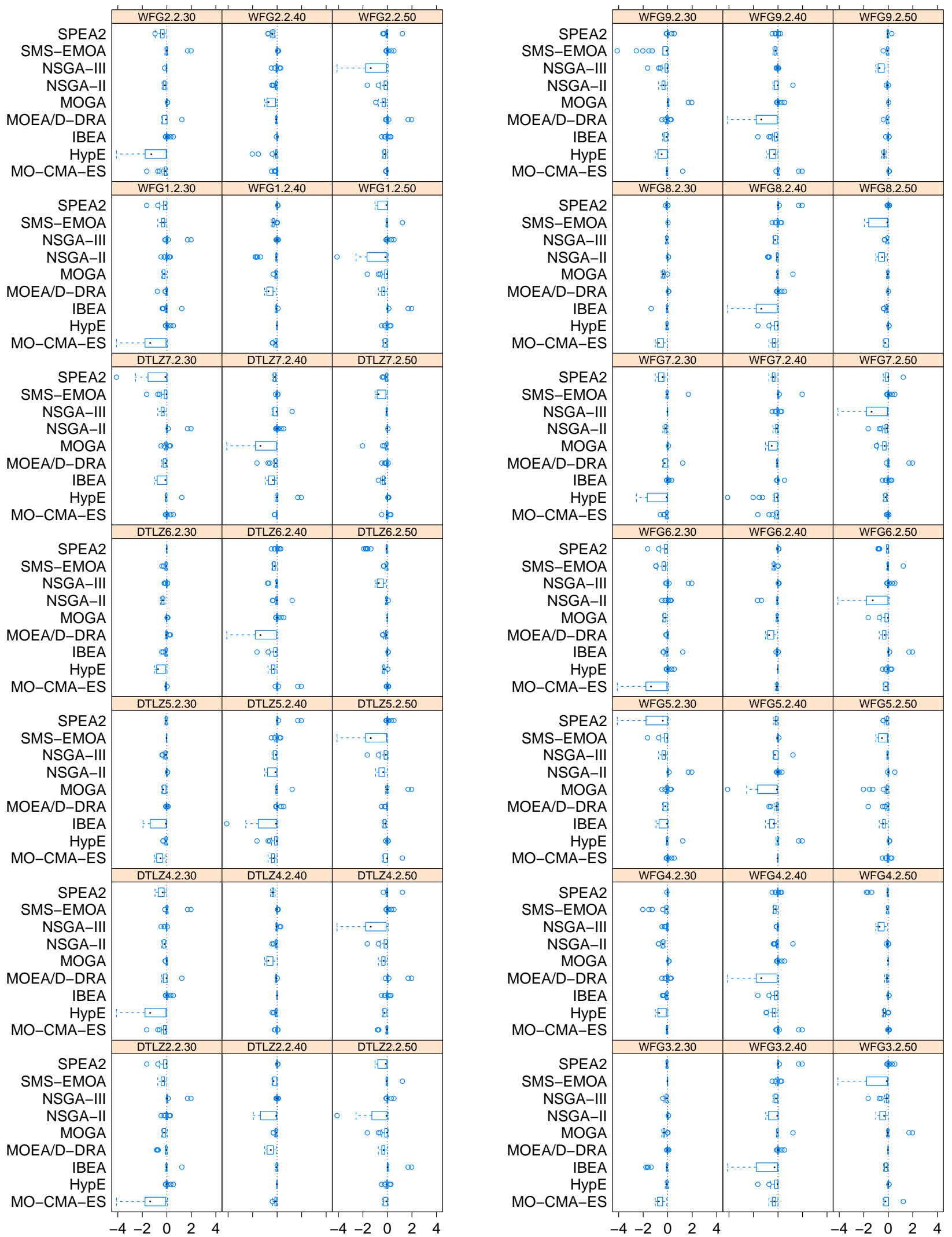

Figure 41: Performance differences according to $I_{I G D}$ between runs from MOEAs with and without unbounded archiving on the scenario with $M=2$ and $\boldsymbol{F E}_{\mathbf{m a x}}=2500$ for all problems with $n_{\mathrm{var}} \in\{30,40,50\}$. 

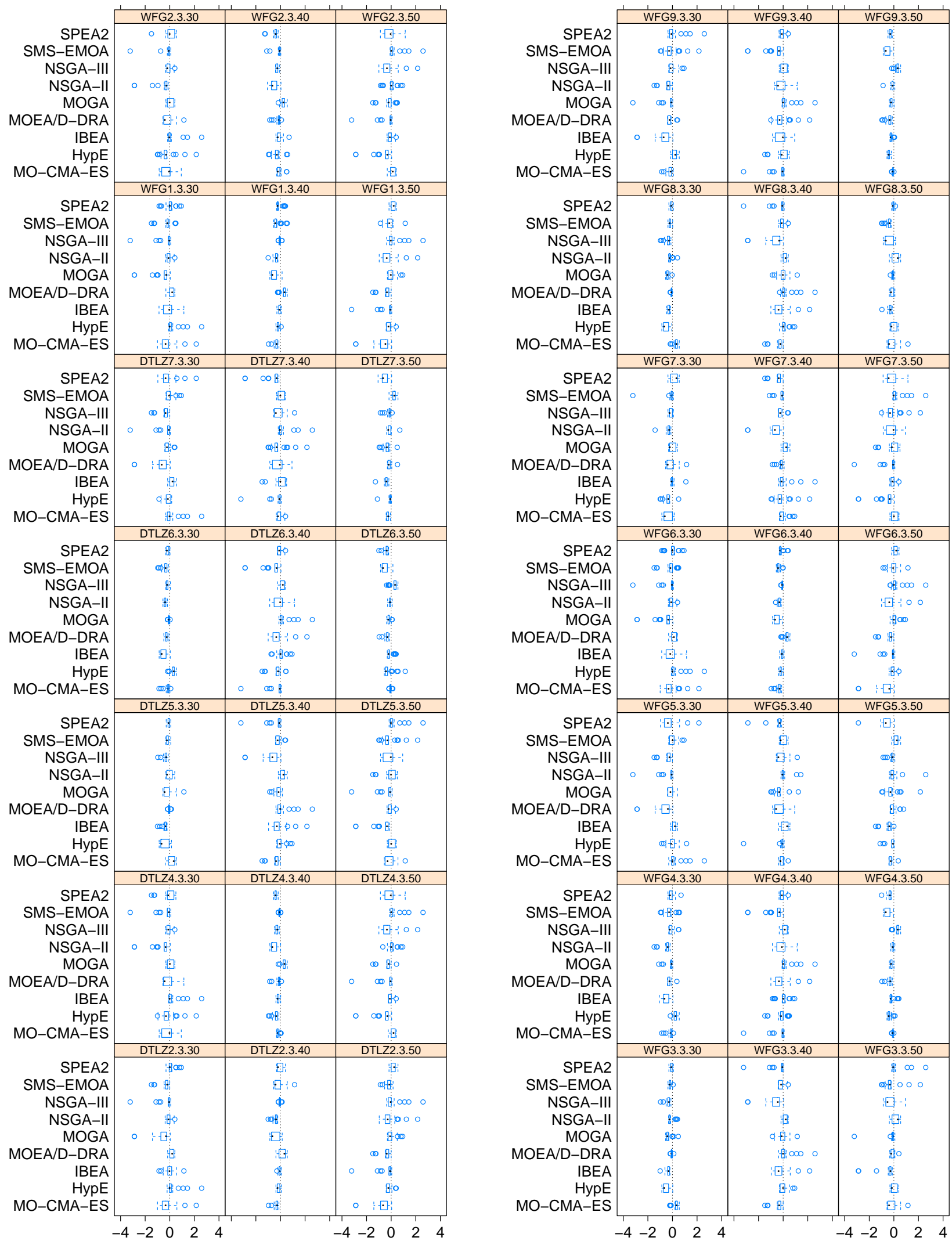

Figure 42: Performance differences according to $I_{I G D}$ between runs from MOEAs with and without unbounded archiving on the scenario with $M=3$ and $\boldsymbol{F E}_{\mathbf{m a x}}=2500$ for all problems with $n_{\mathrm{var}} \in\{30,40,50\}$. 

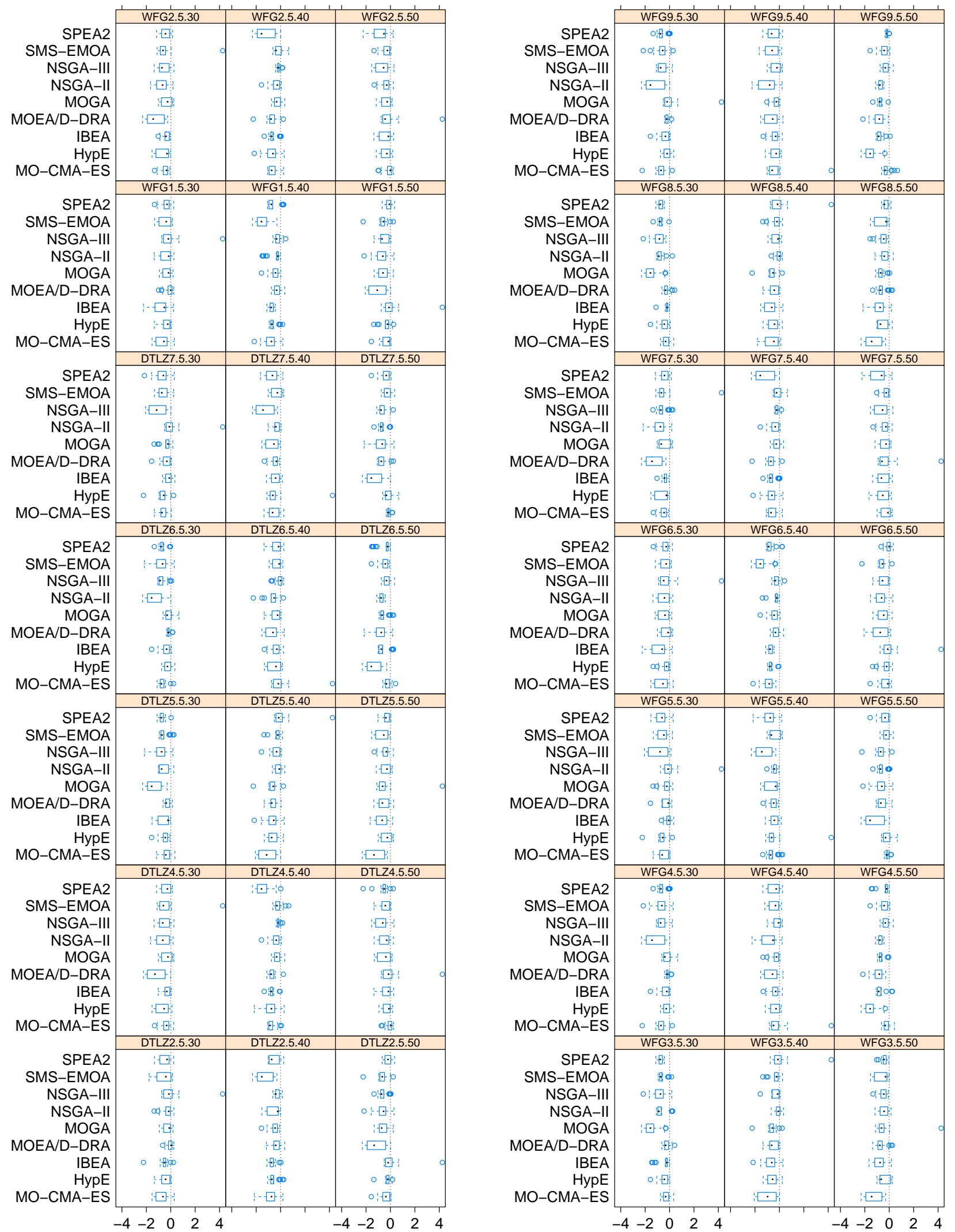

Figure 43: Performance differences according to $I_{I G D}$ between runs from MOEAs with and without unbounded archiving on the scenario with $M=5$ and $\boldsymbol{F E}_{\mathbf{m a x}}=2500$ for all problems with $n_{\mathrm{var}} \in\{30,40,50\}$. 

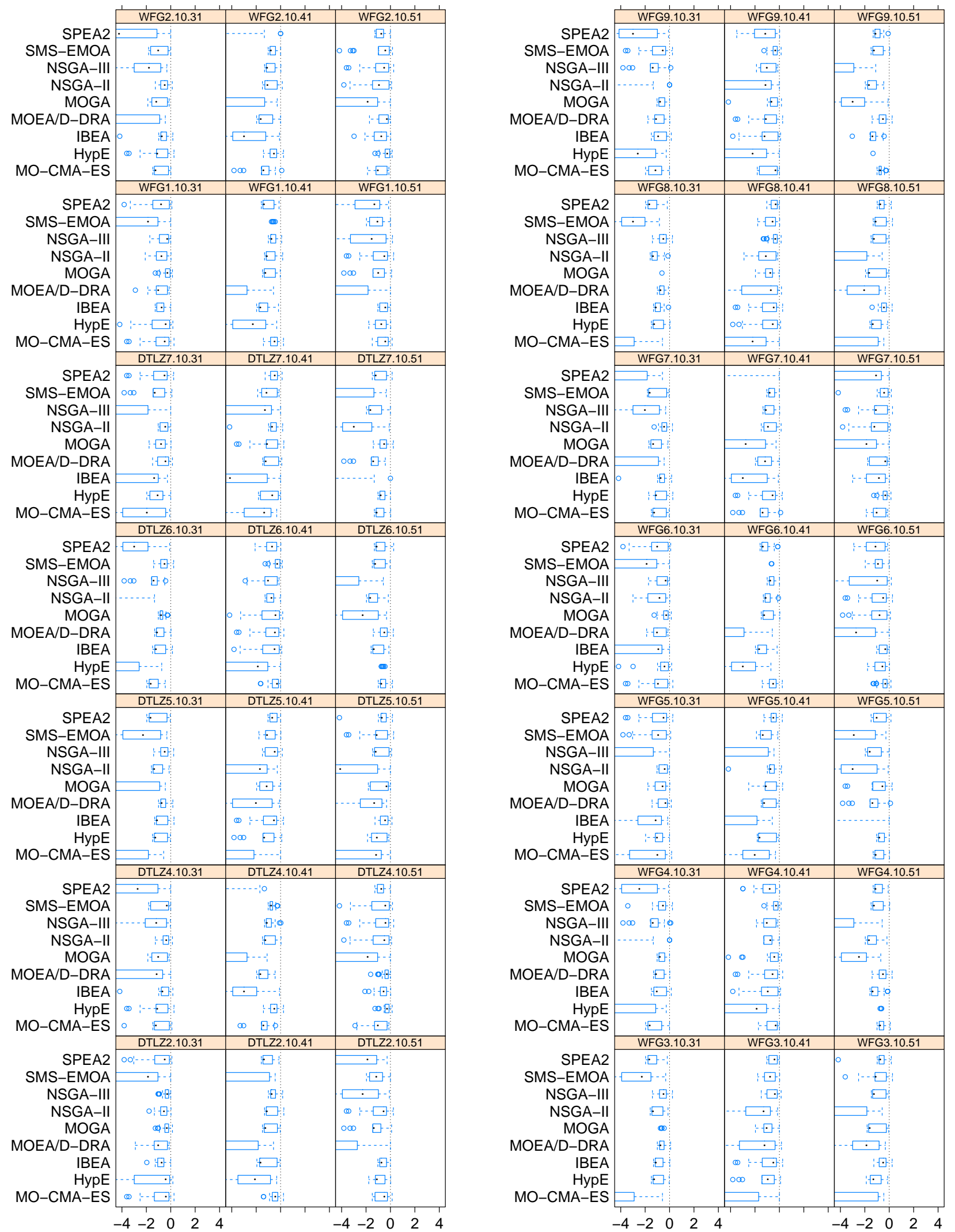

Figure 44: Performance differences according to $I_{I G D}$ between runs from MOEAs with and without unbounded archiving on the scenario with $M=10$ and $\boldsymbol{F E}_{\mathbf{m a x}}=2500$ for all problems with $n_{\mathrm{var}} \in\{30,40,50\}$. 

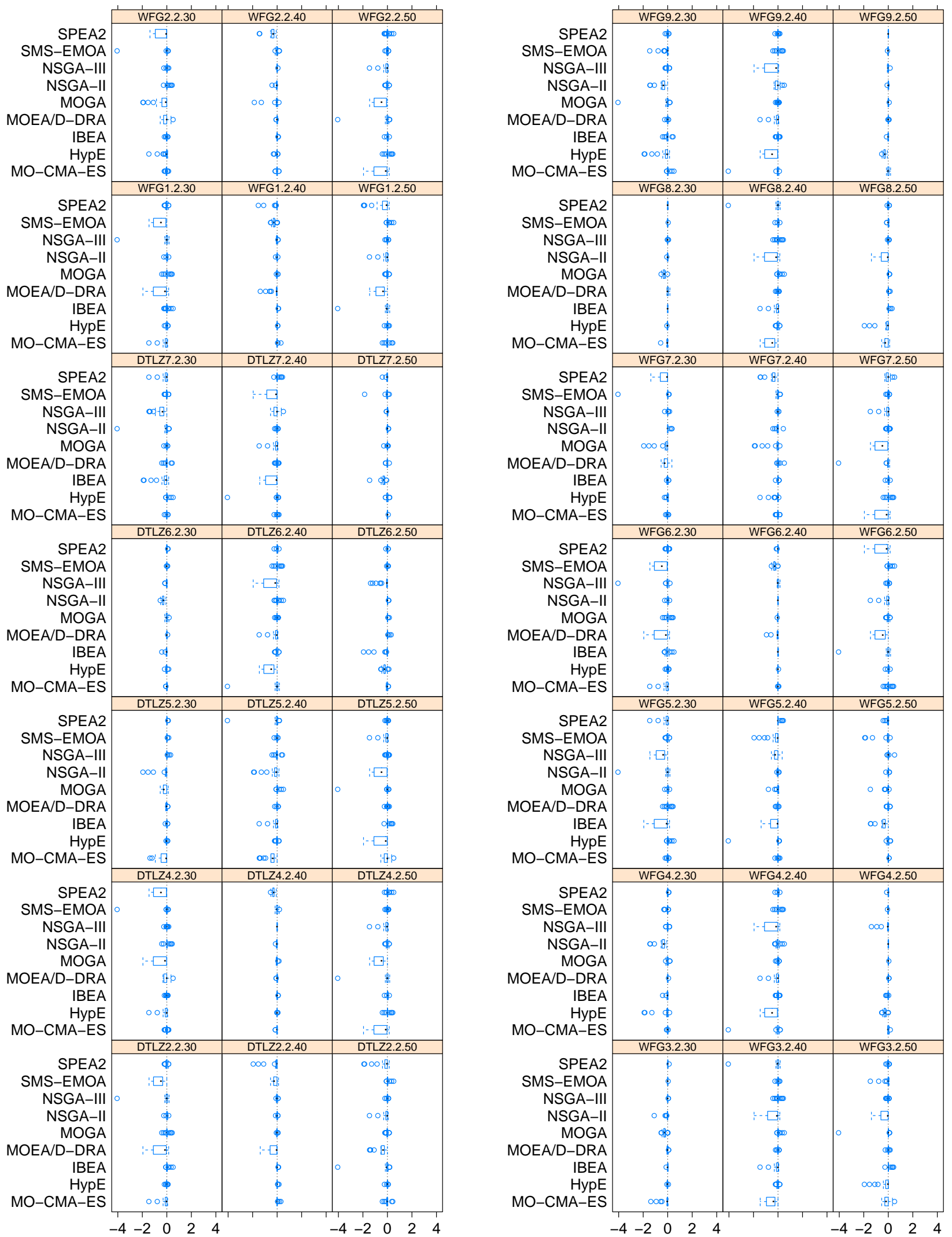

Figure 45: Performance differences according to $I_{I G D}$ between runs from MOEAs with and without unbounded archiving on the scenario with $M=2$ and $\boldsymbol{F E}_{\max }=10000$ for all problems with $n_{\mathrm{var}} \in\{30,40,50\}$. 

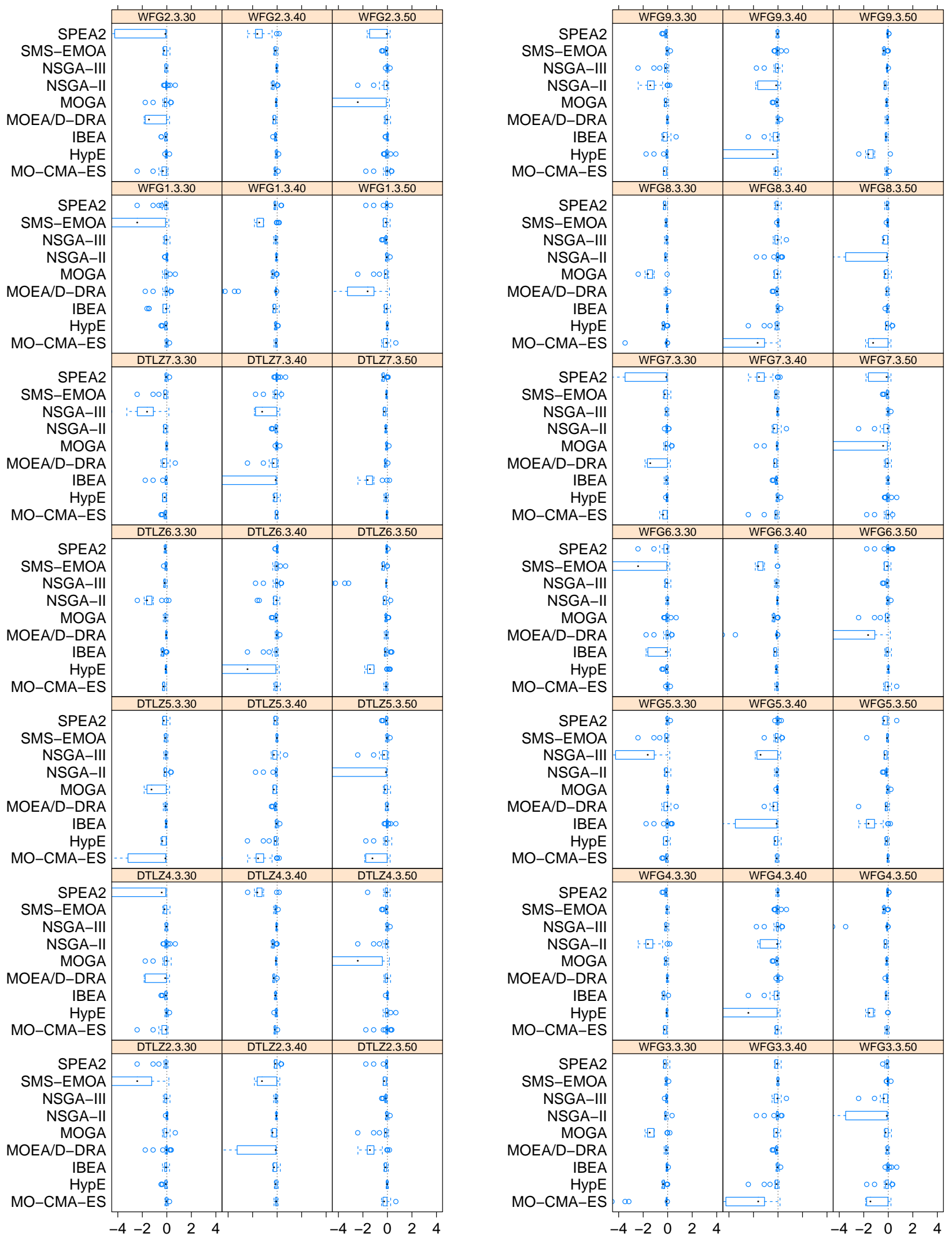

Figure 46: Performance differences according to $I_{I G D}$ between runs from MOEAs with and without unbounded archiving on the scenario with $M=3$ and $\boldsymbol{F E}_{\max }=10000$ for all problems with $n_{\mathrm{var}} \in\{30,40,50\}$. 

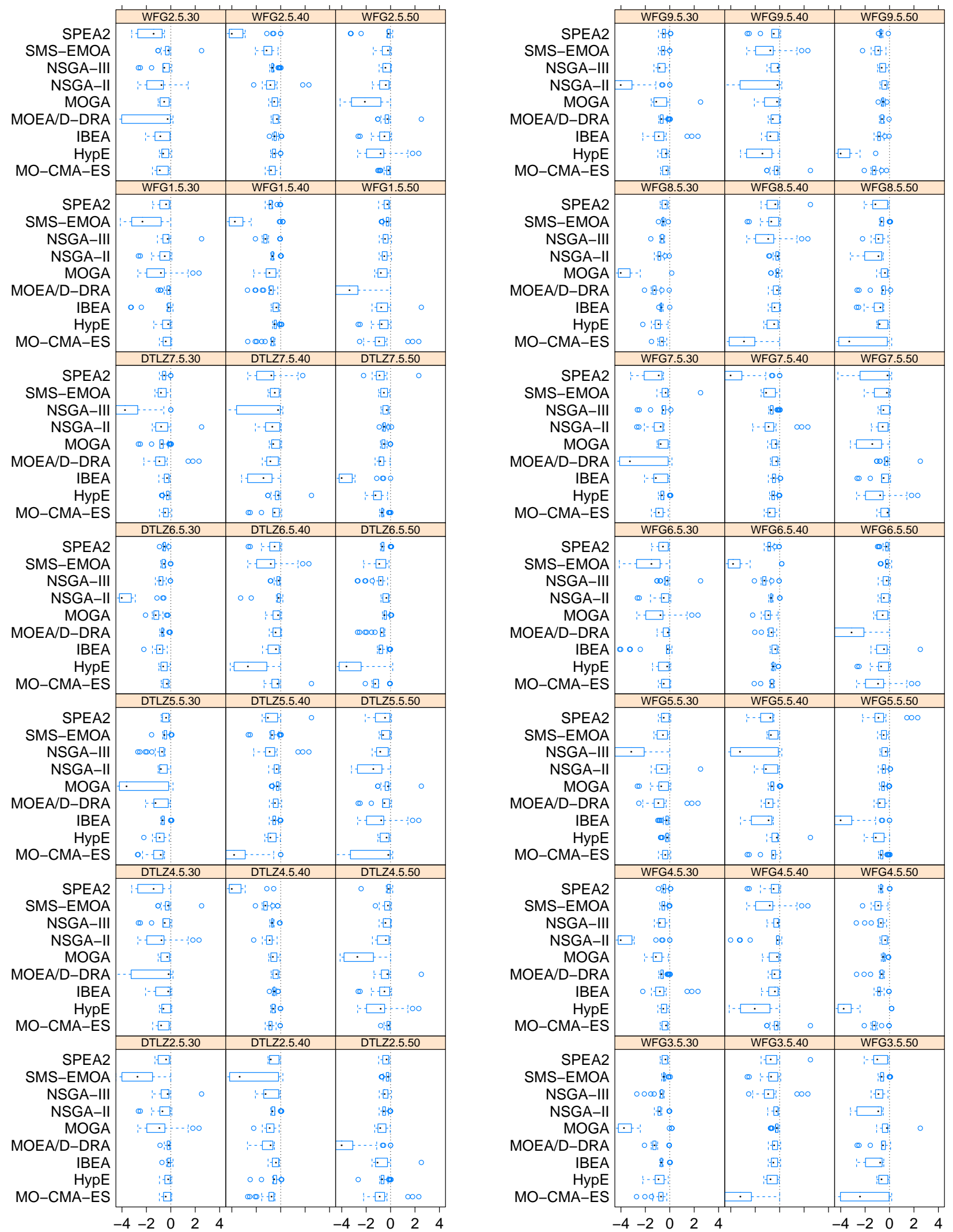

Figure 47: Performance differences according to $I_{I G D}$ between runs from MOEAs with and without unbounded archiving on the scenario with $M=5$ and $\boldsymbol{F E}_{\max }=10000$ for all problems with $n_{\mathrm{var}} \in\{30,40,50\}$. 

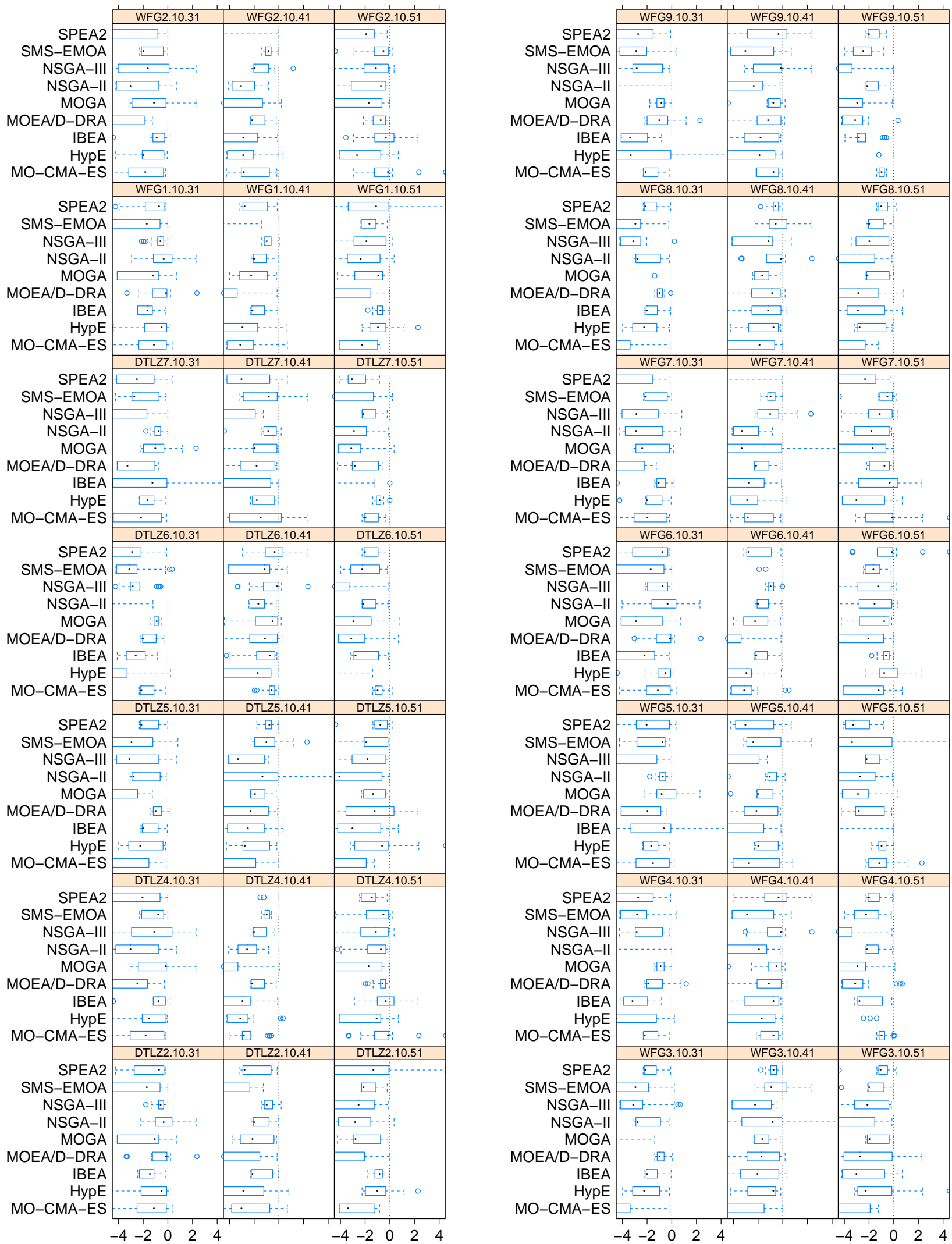

Figure 48: Performance differences according to $I_{I G D}$ between runs from MOEAs with and without unbounded archiving on the scenario with $M=10$ and $\boldsymbol{F E}_{\mathbf{m a x}}=10000$ for all problems with $n_{\mathbf{v a r}} \in\{30,40,50\}$. 

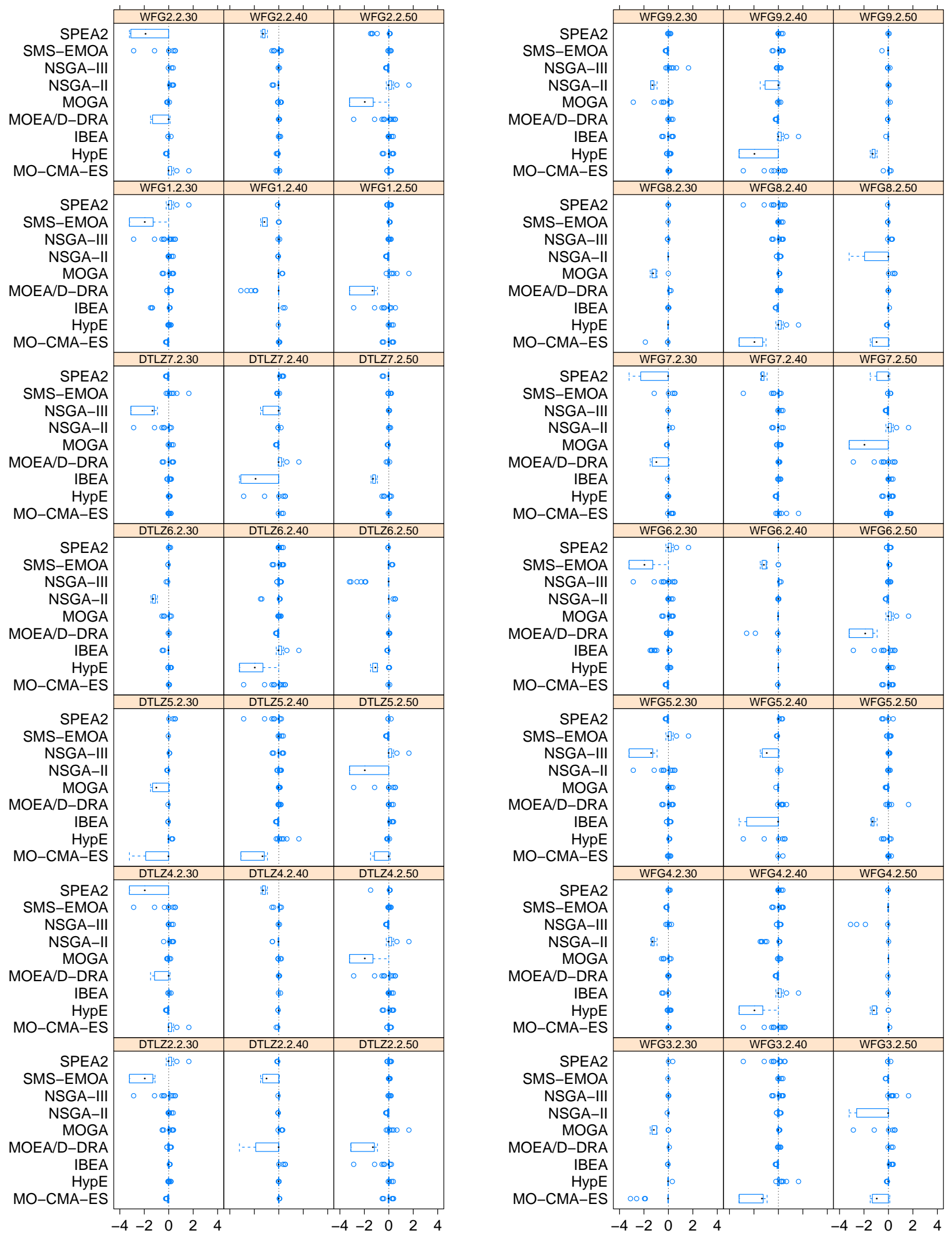

Figure 49: Performance differences according to $I_{I G D}$ between runs from MOEAs with and without unbounded archiving on the scenario with $M=2$ and $\boldsymbol{F E}_{\max }=40000$ for all problems with $n_{\mathrm{var}} \in\{30,40,50\}$. 

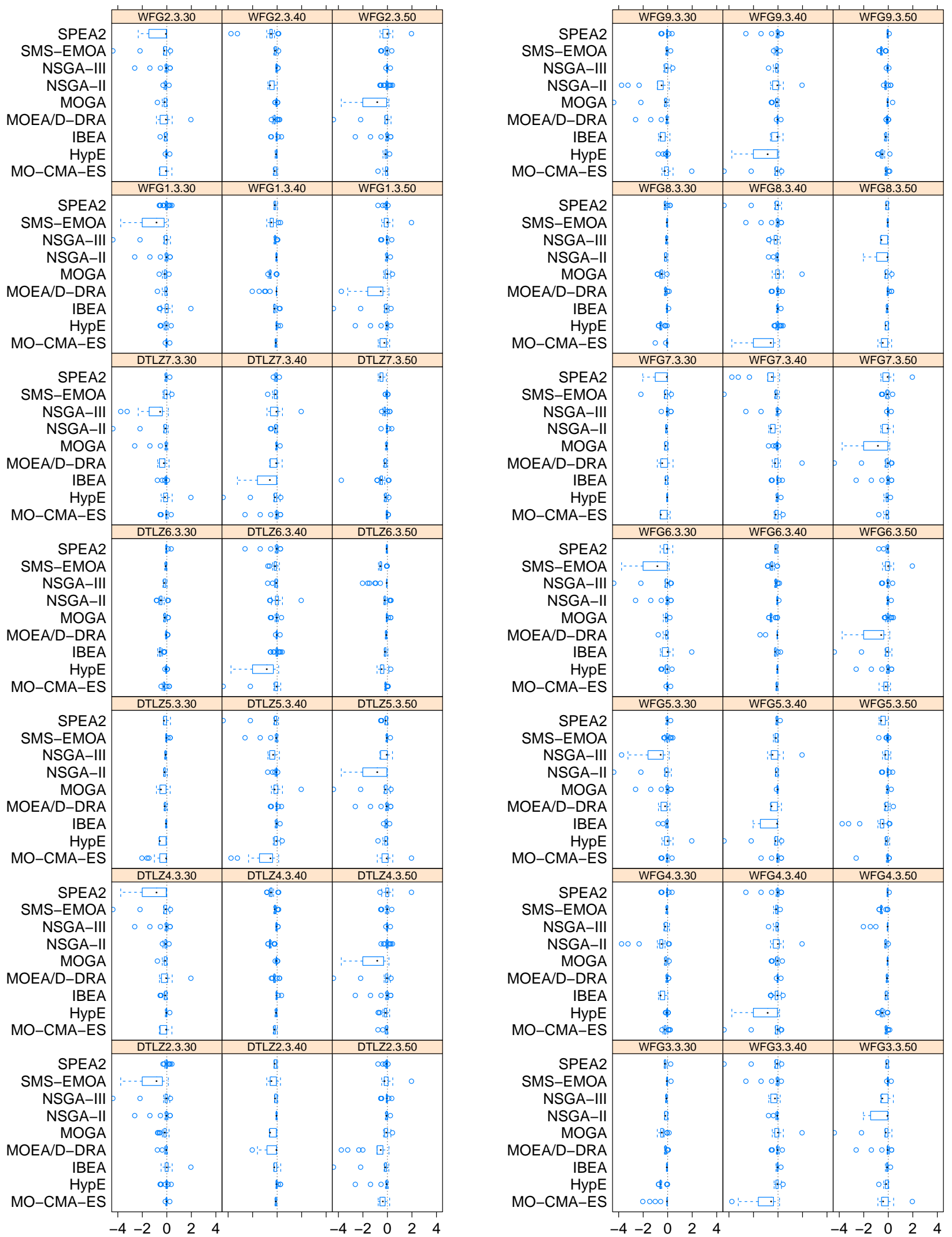

Figure 50: Performance differences according to $I_{I G D}$ between runs from MOEAs with and without unbounded archiving on the scenario with $M=3$ and $\boldsymbol{F E}_{\max }=40000$ for all problems with $n_{\mathrm{var}} \in\{30,40,50\}$. 

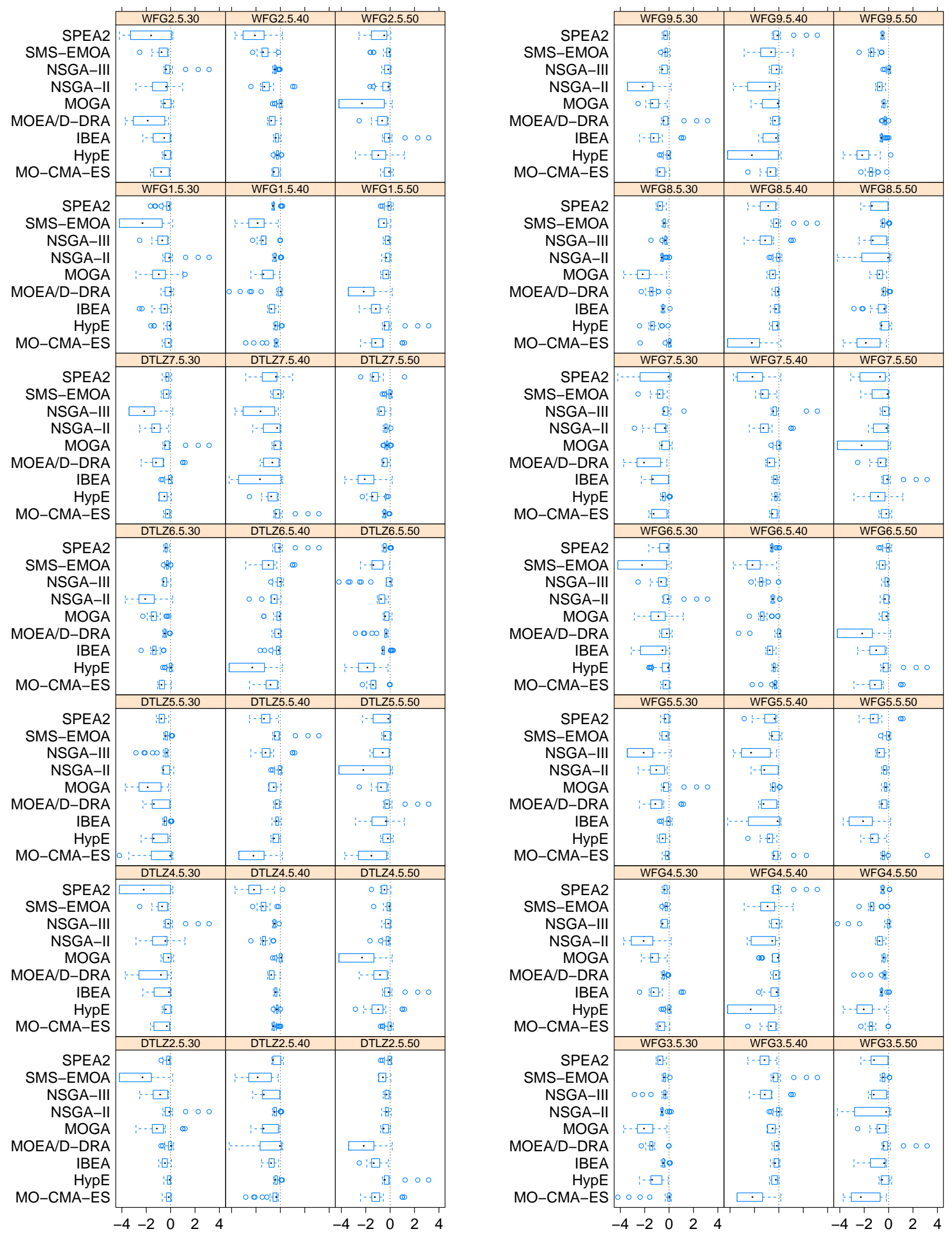

Figure 51: Performance differences according to $I_{I G D}$ between runs from MOEAs with and without unbounded archiving on the scenario with $M=5$ and $\boldsymbol{F E}_{\max }=40000$ for all problems with $n_{\mathrm{var}} \in\{30,40,50\}$. 

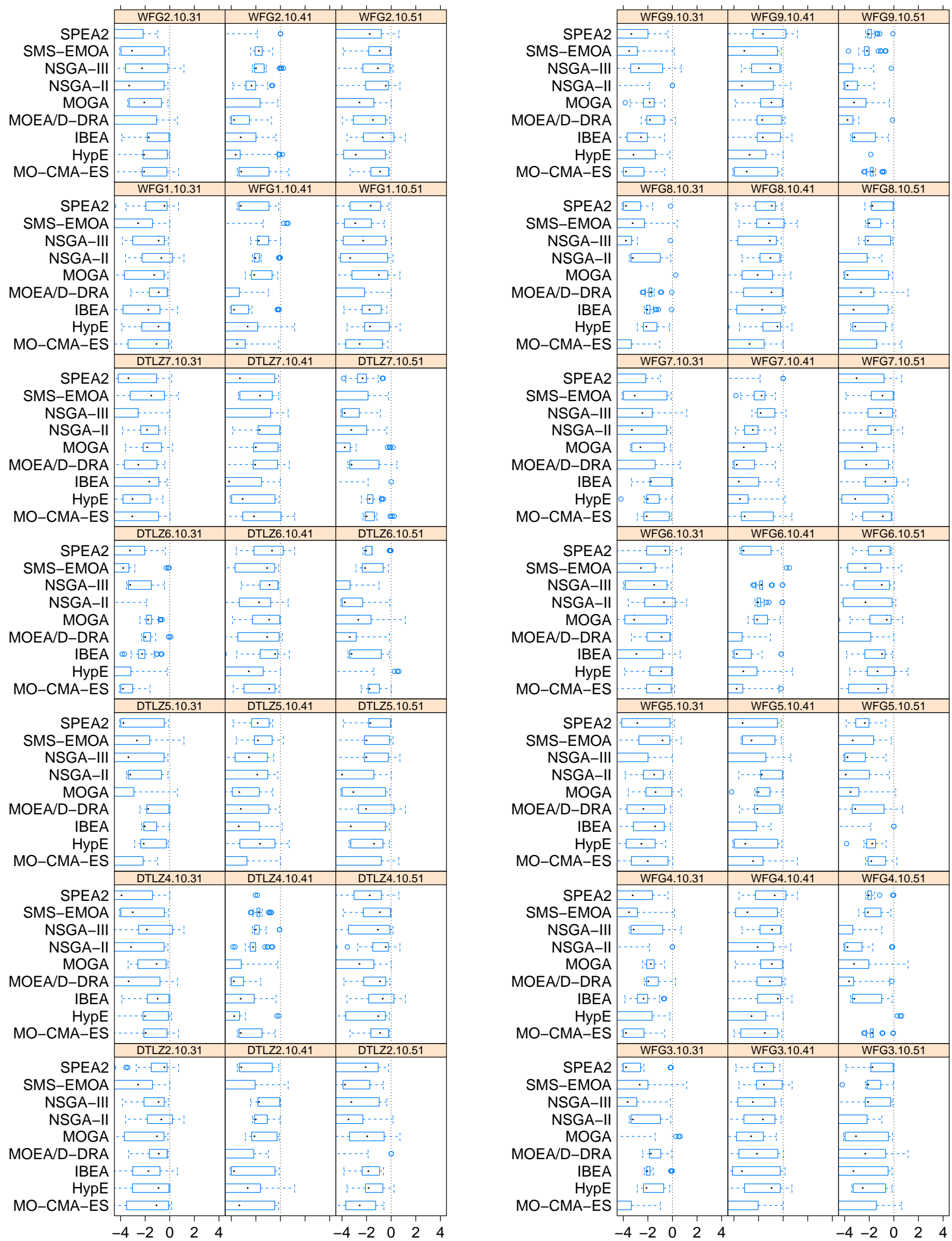

Figure 52: Performance differences according to $I_{I G D}$ between runs from MOEAs with and without unbounded archiving on the scenario with $M=10$ and $\boldsymbol{F E}_{\mathbf{m a x}}=40000$ for all problems with $n_{\mathbf{v a r}} \in\{30,40,50\}$. 

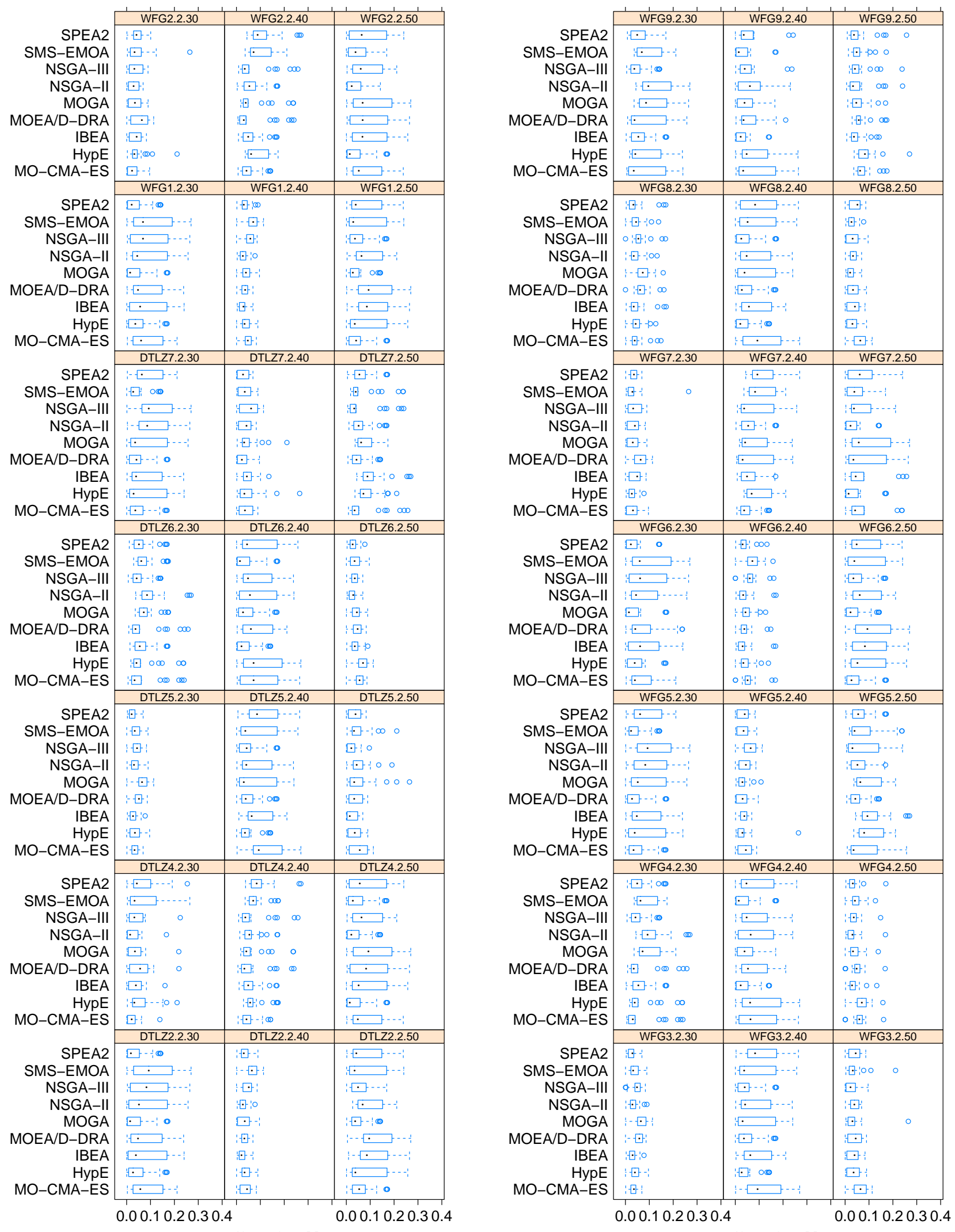

Figure 53: Boxplots of the $I_{H}^{\boldsymbol{r d}}$ for $M=2$ problems, $n_{\mathbf{v a r}} \in\{30,40,50\}$ and $\boldsymbol{F} \boldsymbol{E}_{\mathbf{m a x}}=2500$. 

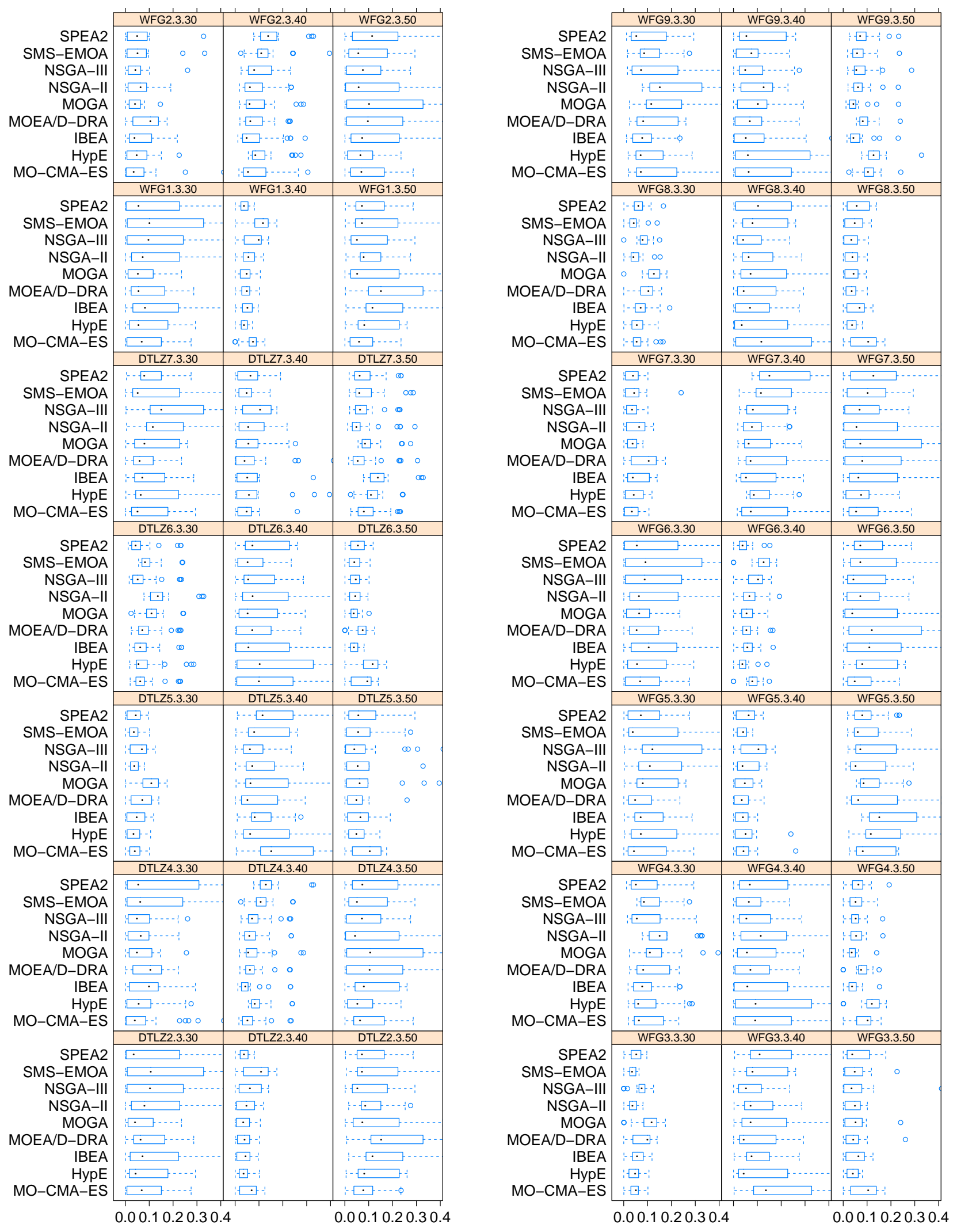

Figure 54: Boxplots of the $I_{H}^{\boldsymbol{r d}}$ for $M=3$ problems, $n_{\mathbf{v a r}} \in\{30,40,50\}$ and $\boldsymbol{F E}_{\mathbf{m a x}}=2500$. 

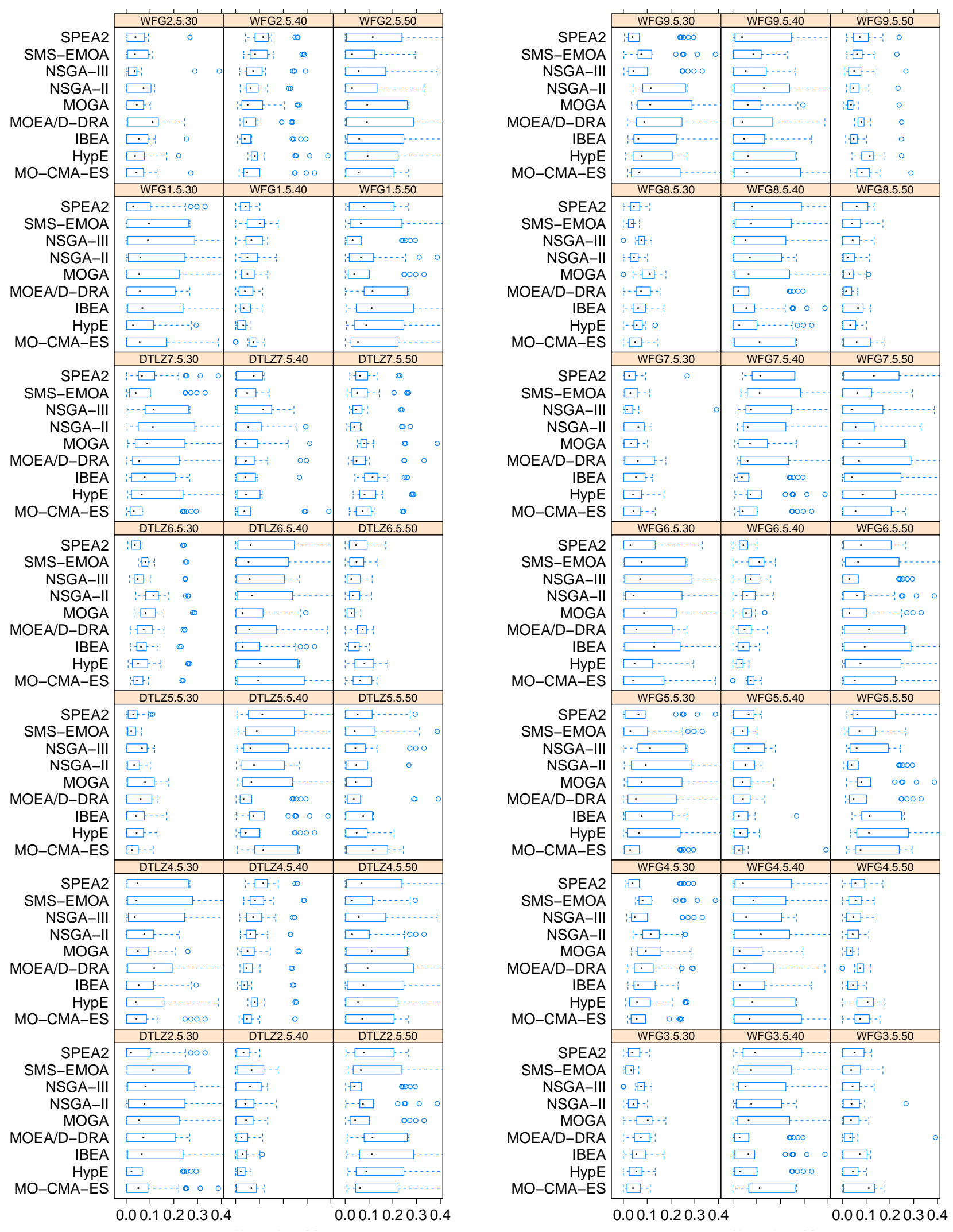

Figure 55: Boxplots of the $I_{H}^{\boldsymbol{r d}}$ for $M=5$ problems, $n_{\mathbf{v a r}} \in\{30,40,50\}$ and $\boldsymbol{F} \boldsymbol{E}_{\mathbf{m a x}}=2500$. 

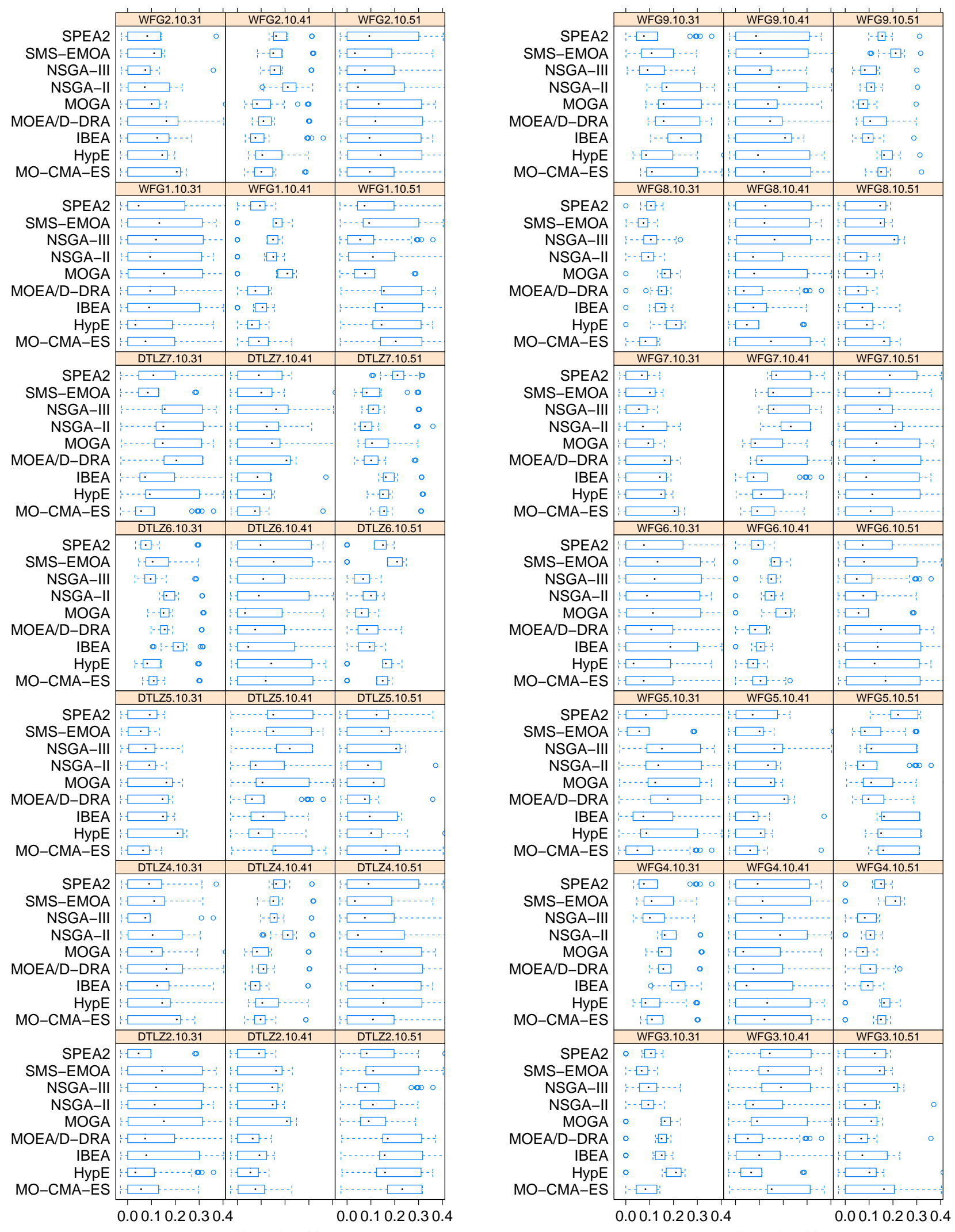

Figure 56: Boxplots of the $I_{H}^{r d}$ for $M=10$ problems, $n_{\mathbf{v a r}} \in\{30,40,50\}$ and $\boldsymbol{F} E_{\max }=2500$. 

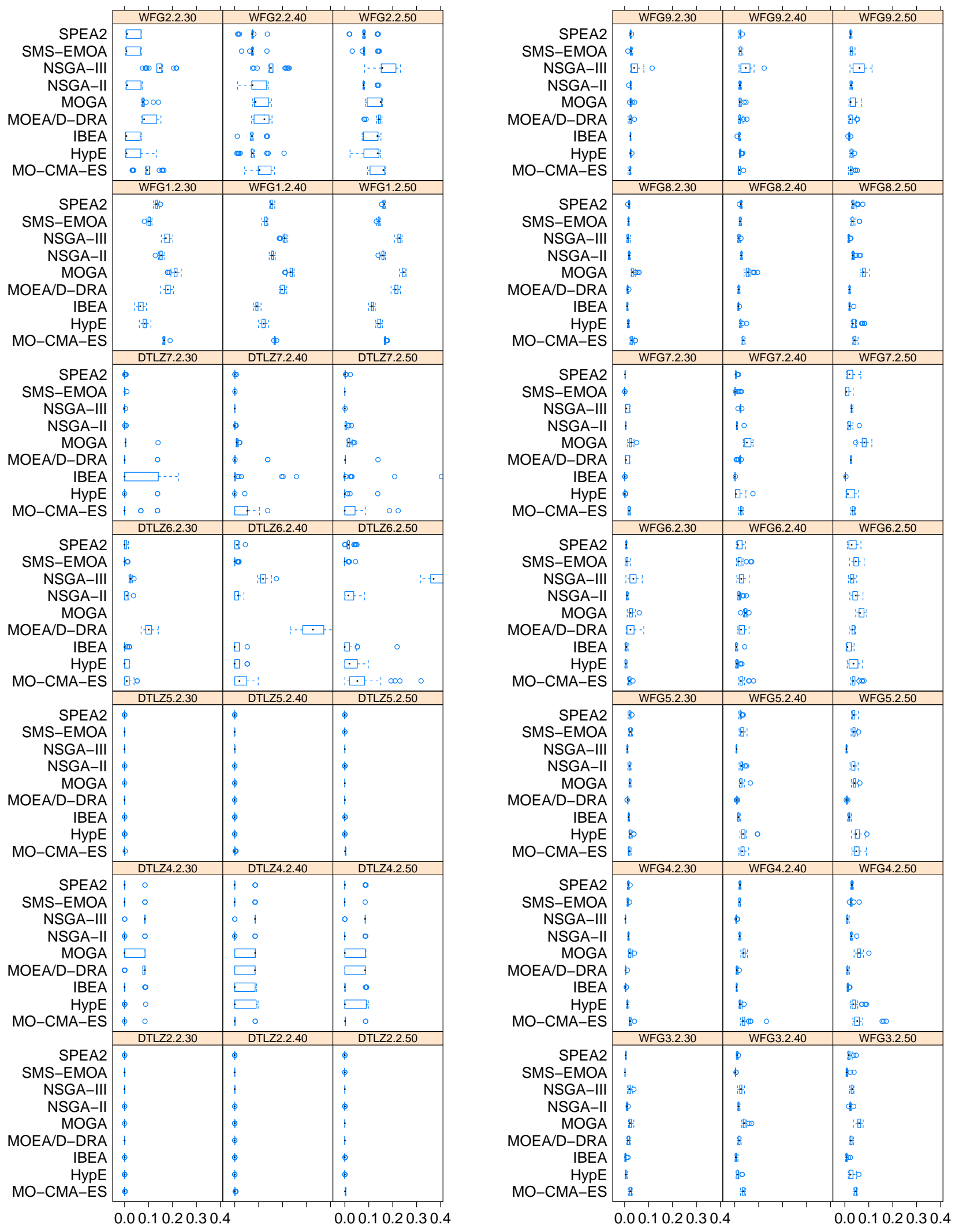

Figure 57: Boxplots of the $I_{H}^{\boldsymbol{r d}}$ for $M=2$ problems, $n_{\mathbf{v a r}} \in\{30,40,50\}$ and $\boldsymbol{F} \boldsymbol{E}_{\max }=10000$. 

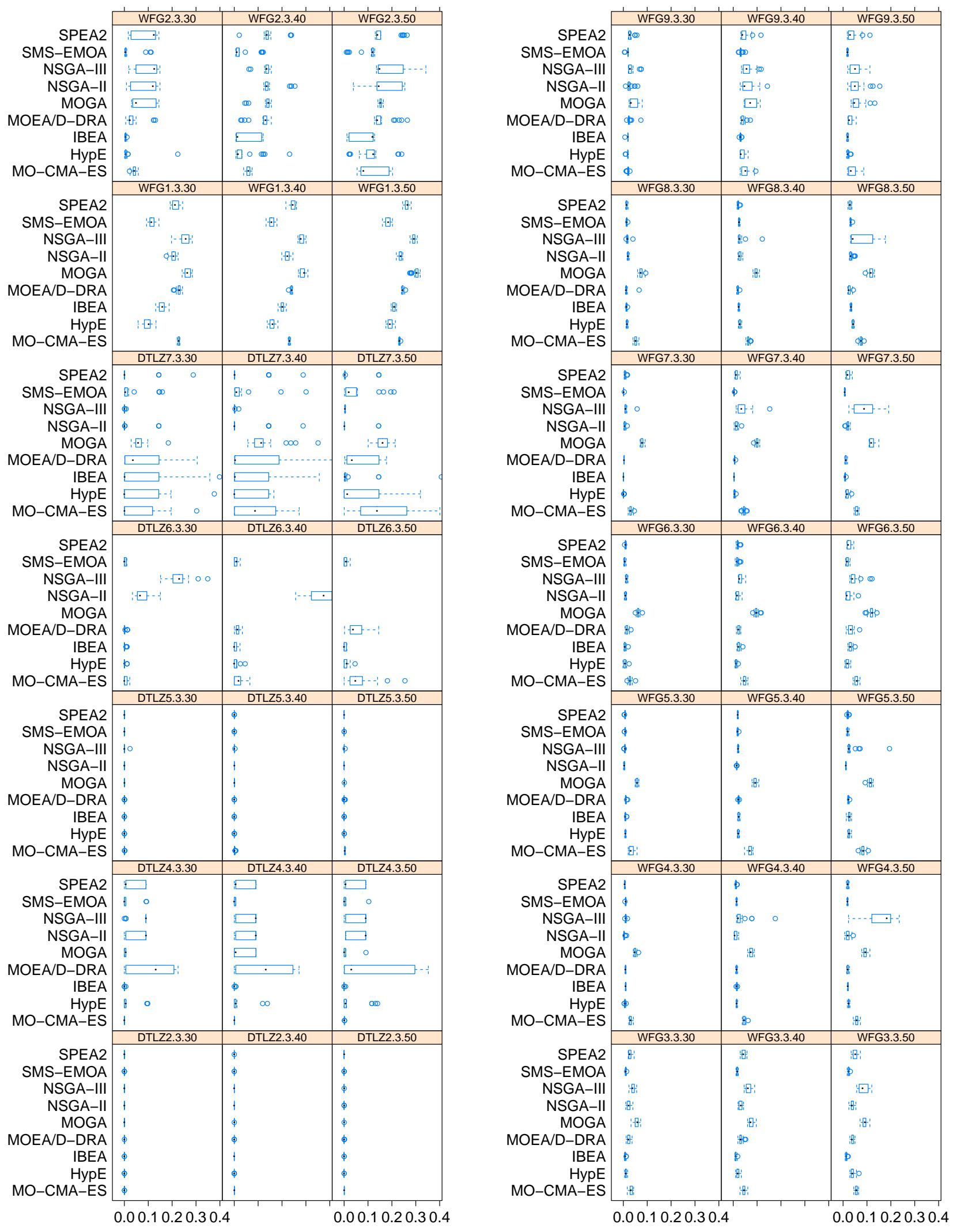

Figure 58: Boxplots of the $I_{H}^{\boldsymbol{r d}}$ for $M=3$ problems, $n_{\mathbf{v a r}} \in\{30,40,50\}$ and $\boldsymbol{F} \boldsymbol{E}_{\max }=10000$. 

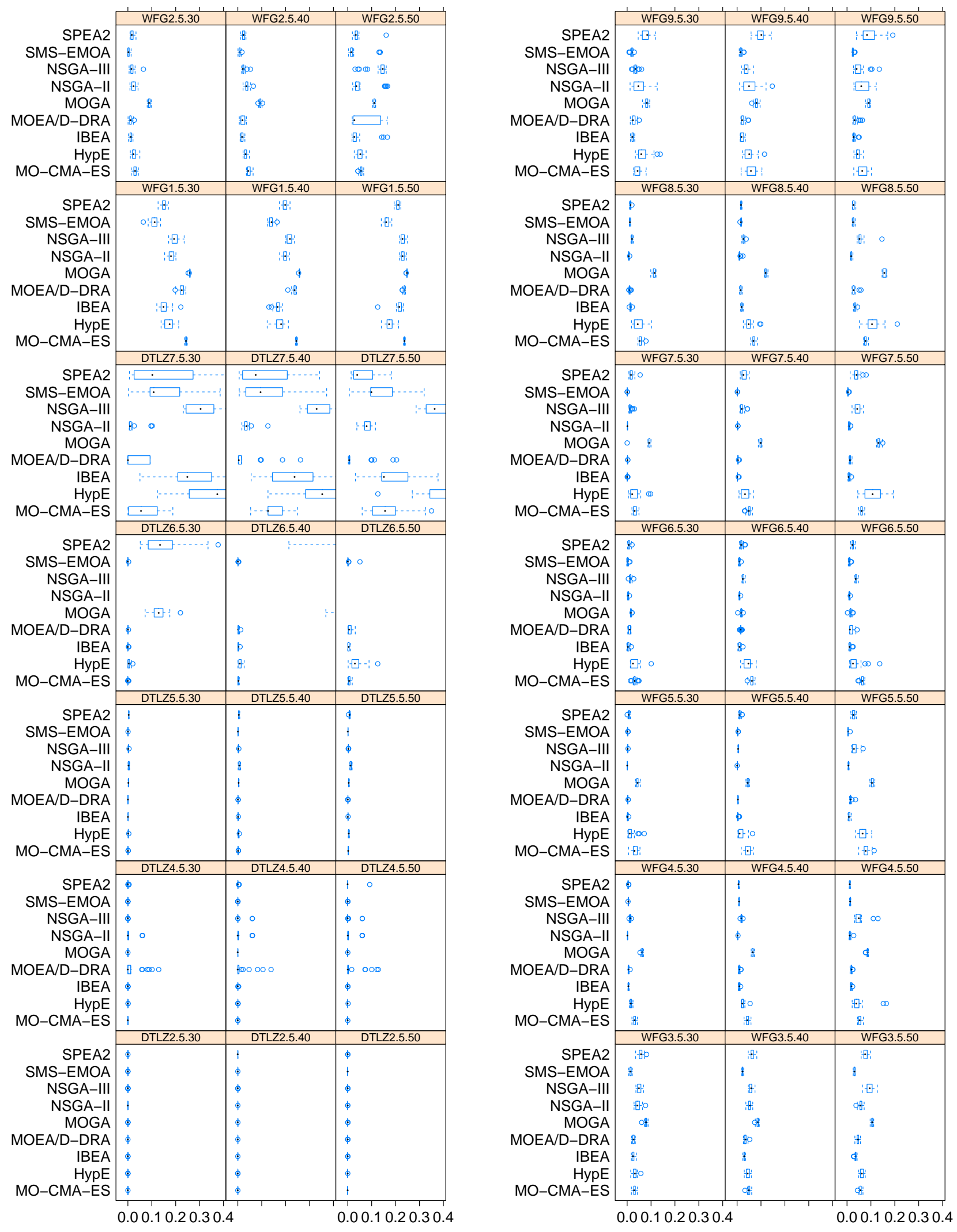

Figure 59: Boxplots of the $I_{H}^{\boldsymbol{r d}}$ for $M=5$ problems, $n_{\mathbf{v a r}} \in\{30,40,50\}$ and $\boldsymbol{F} \boldsymbol{E}_{\max }=10000$. 

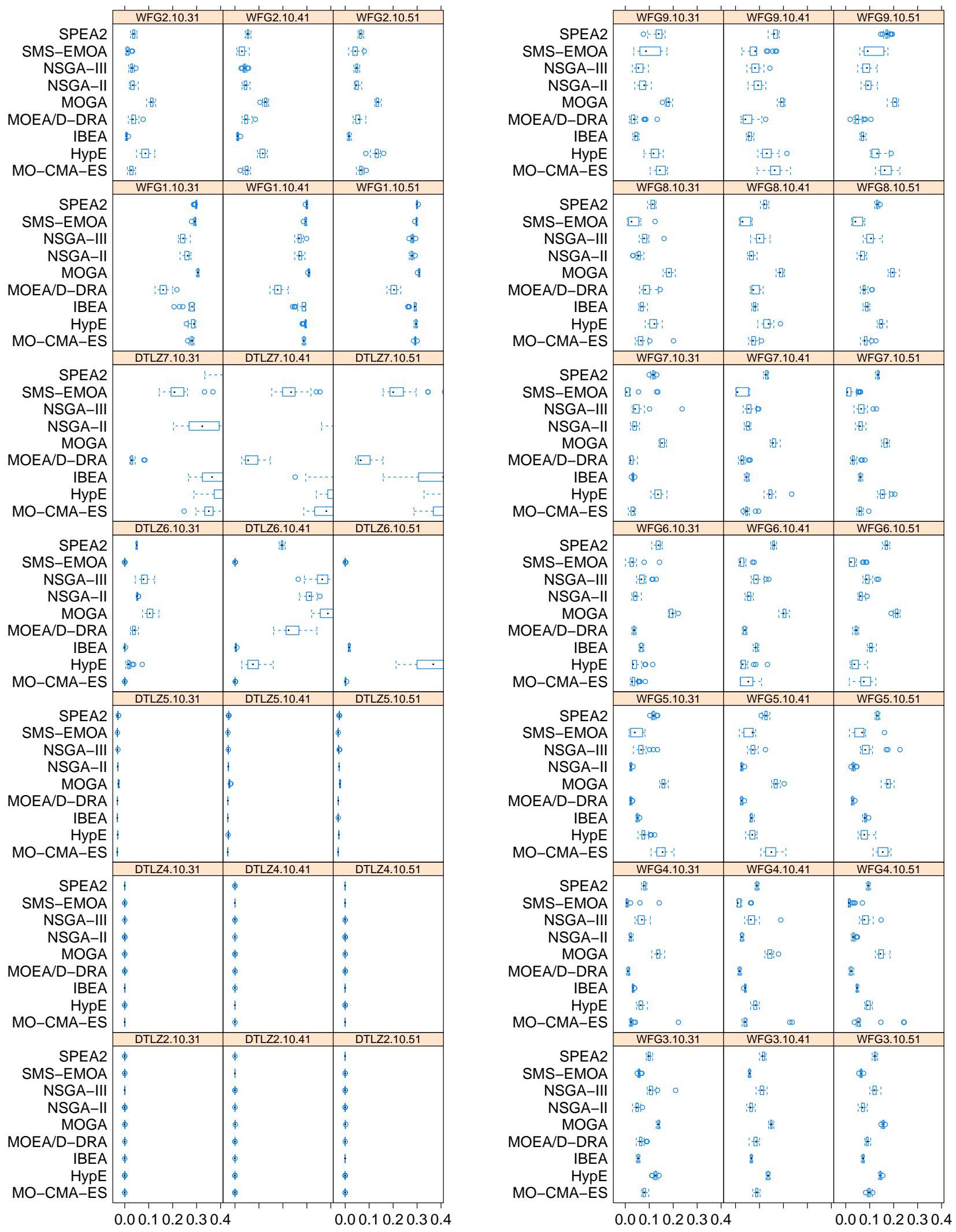

Figure 60: Boxplots of the $I_{H}^{\boldsymbol{r d}}$ for $M=10$ problems, $n_{\mathbf{v a r}} \in\{30,40,50\}$ and $\boldsymbol{F} \boldsymbol{E}_{\max }=10000$. 


\begin{tabular}{|c|c|c|c|}
\hline & 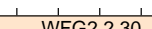 & $\frac{1}{1}$ & WECS250 \\
\hline SPEA2 & Fio o & 700000 & F-1000 o 0 \\
\hline SMS-EMOA & 日。 & 沺 00000 & $\because \div \infty$ \\
\hline NSGA-III & E: & Fit & $\nabla-100$ \\
\hline NSGA-II & E: & 沺：。 & Q \\
\hline MOGA & E由 & 1] 0000 & $\cdot-\cdots$ \\
\hline MOEA/D-DRA & E: & fipo 0000 & $\square^{\cdots-1}$ \\
\hline IBEA & Ep & ⿶® & - $-1 \infty$ \\
\hline HypE & Eio & 沺0000 & E;oo \\
\hline MO-CMA-ES & 6! & $f_{i} \infty$ & F- $-1, \infty$ \\
\hline & WFG1.2.30 & WFG1.2.40 & WFG1.2.50 \\
\hline SPEA2 & F! & flpo & $\nabla-1,000$ \\
\hline SMS-EMOA & $\square^{-\cdots}$ & 沼: & $8: 0000$ \\
\hline NSGA-III & $\cdot \nabla^{-\cdots}$ & F: & 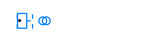 \\
\hline NSGA-II & $7 \div$ & 日 & 田: \\
\hline MOGA & Ei 00 & 洇: & f:co \\
\hline MOEA/D-DRA & $\square-1, \infty$ & E:0 & {$\left[\begin{array}{l}-1 \\
0\end{array}\right.$} \\
\hline IBEA & ए:-000 0 & 㥸。 & $\square^{--10}$ \\
\hline HypE & 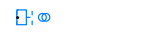 & F: & $F+\infty$ \\
\hline MO-CMA-ES & $\nabla-100$ & 海 & Q: $: 0$ \\
\hline & DTLZ7.2.30 & DTLZ7.2.40 & DTLZ7.2.50 \\
\hline SPEA2 & 000 & Fi & 情: 10 \\
\hline SMS-EMOA & $8 \infty$ & E申 & (1) $\infty 000$ \\
\hline NSGA-III & $\square-\cdots$ & E: & $\mathbb{F}: 0000$ \\
\hline NSGA-II & 日00000 & Ep & Fi \\
\hline MOGA & Gion & Eio & 㽖0000 \\
\hline MOEA/D-DRA & F:0 & 하 & $H_{i} \infty$ \\
\hline IBEA & $-1,000$ & घ:0 0 & 讨 00000 \\
\hline HypE & ⿴囗十 00000 & E。 & 调 000000 \\
\hline MO-CMA-ES & घ;œ & 暗 & 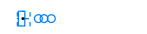 \\
\hline & DTLZ6.2.30 & DTLZ6.2.40 & DTLZ6.2.50 \\
\hline SPEA2 & S & 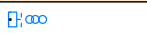 & $9 \%$ \\
\hline SMS-EMOA & 㥜0000 & 800 & H: \\
\hline NSGA-III & $f p \infty$ & {$\left[\begin{array}{l}-\infty \\
-\infty\end{array}\right.$} & 8:0 \\
\hline NSGA-II & it $00 \infty \infty$ & E; 0000 & H. 0 \\
\hline MOGA & F00000 & 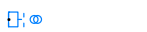 & F \\
\hline MOEA/D-DRA & $\mathbb{B}, \infty$ & D-100 & 湈 \\
\hline IBEA & 酒 & & B: \\
\hline HypE & $\theta \infty$ & $\cdot \nabla^{\cdots}$ & 细: \\
\hline MO-CMA-ES & int & $\varphi^{-\cdots}$ & G: \\
\hline & DTLZ5.2.30 & DTLZ5.2.40 & DTLZ5.2.50 \\
\hline SPEA2 & Bit 0 & it 000000 & Ep \\
\hline SMS-EMOA & 淂: & Gito & E: \\
\hline NSGA-III & if: & 日:0 & $1-\frac{1}{1}$ \\
\hline NSGA-II & E: & -1,00 & E:0 0 \\
\hline MOGA & 淇: & fit;, 0000 & E 0 \\
\hline MOEA/D-DRA & Ep & 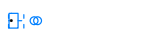 & E: \\
\hline IBEA & EH & 000 & E: \\
\hline HypE & 因: & & E申 \\
\hline MO-CMA-ES & B: & 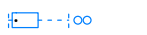 & E: \\
\hline & DTLZ4.2.30 & DTLZ4.2.40 & DTLZ4.2.50 \\
\hline SPEA2 & Fio $\infty$ & 讯 00000 & घ:0000 ० \\
\hline SMS-EMOA & 日; $\infty$ & 浬 0000 & Fpo \\
\hline NSGA-III & E: & Fico & ०-:०० \\
\hline NSGA-II & F: & 泡 & $\theta-1$ \\
\hline MOGA & E巾。 & Dis 0000 & $\cdot-\cdots$ \\
\hline MOEA/D-DRA & E:0 & ig: & 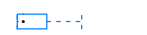 \\
\hline IBEA & & 泇必 & E:co \\
\hline HypE & E: 0 & : & घ०० \\
\hline MO-CMA-ES & & $f ; \infty$ & $\nabla-1, \infty$ \\
\hline & DTLZ2.2.30 & DTLZ2.2.40 & DTLZ2.2.50 \\
\hline SPEA2 & $\forall \div$ & B: & : \\
\hline SMS-EMOA & 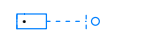 & : & fitiono \\
\hline NSGA-III & $\square^{-\cdots}$ & GP & Hळ \\
\hline NSGA-II & $0 ; 0 \infty$ & 日: & 000 \\
\hline MOGA & 800 & E: & $8 \infty$ \\
\hline MOEA/D-DRA & $\square-1 \infty$ & E:0 & $\square^{--100}$ \\
\hline IBEA & E:0000 0 & [H。 & $\theta \div 0000$ \\
\hline HypE & H:Oळ & E: & 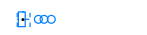 \\
\hline MO-CMA-ES & $0-100$ & 蝴 & 田; 0 \\
\hline & 0.00 .10 .2 & & $0.00 .10 .2 C$ \\
\hline
\end{tabular}

\begin{tabular}{|c|c|c|c|}
\hline & WFG9.2.30 & WFG9.2.40 & WFG9.2.50 \\
\hline SPEA2 & $\forall \infty$ & 日。 & Hi $\infty$ \\
\hline SMS-EMOA & 田: & GiO & 期po \\
\hline NSGA-III & $8 \infty$ & ज: $: 0$ & 蛅 0000 \\
\hline NSGA-II & $\forall-\cdots$ & Eto 0 & 活: $\infty$ \\
\hline MOGA & 证 $\div$ †000 & Eto & 泇œ \\
\hline MOEA/D-DRA & E:co & E: 0 & 䍐0 0000 \\
\hline IBEA & 计: : & Fto & $F_{1} \infty$ \\
\hline HypE & $\because-1, \infty \infty$ & $\square \div \infty$ & 闽 000 ○ \\
\hline \multirow[t]{2}{*}{ MO-CMA-ES } & 舴 00000 & 曰 $\infty \circ$ & 渴: $0 \infty$ \\
\hline & WFG8.2.30 & WFG8.2.40 & WFG8.2.50 \\
\hline SPEA2 & 酚 $\infty$ & $\square-\cdots$ & Ep \\
\hline SMS-EMOA & 络: & 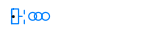 & E: \\
\hline NSGA-III & : & 800 & Gi \\
\hline NSGA-II & $\because ; \infty$ & $\square-1 \infty$ & E:O \\
\hline MOGA & 伊: 000 & 甘;0000 0 & H. 0 \\
\hline MOEA/D-DRA & 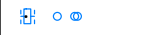 & 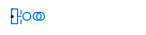 & E: \\
\hline IBEA & BiO & $\nabla-100$ & 趾 \\
\hline HypE & 河：○ & & E: \\
\hline \multirow{2}{*}{ MO-CMA-ES } & 泡 $\infty$ & $\cdot \nabla^{-\cdots}$ & E: \\
\hline & WFG7.2.30 & WFG7.2.40 & WFG7.2.50 \\
\hline SPEA2 & E:0 & $\square-1,00$ & 曰: \\
\hline SMS-EMOA & E: 0 & 诃 † & E:० \\
\hline NSGA-III & E: & Fin & F: 0 \\
\hline NSGA-II & 洞 & 证: 0 & Fio \\
\hline MOGA & E:Ф & t-1,000 & D: $\infty_{0}$ \\
\hline MOEA/D-DRA & Is: & Hit; 00000 & 甘-10०० \\
\hline IBEA & Gp & 曰®⿻ & 배 \\
\hline HypE & E: & 讨 & F:0 \\
\hline \multirow[t]{2}{*}{ MO-CMA-ES } & E: & $8 \infty \infty$ & $\forall-1, \infty$ \\
\hline & WFG6.2.30 & WFG6.2.40 & WFG6.2.50 \\
\hline SPEA2 & Fio & Rp $\infty$ & $\square-100$ \\
\hline SMS-EMOA & 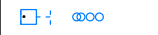 & 谓 00 & f:0000 o \\
\hline NSGA-III & $\nabla-\div 0$ & 䀦 $\infty$ & Fi@ \\
\hline NSGA-II & & Ho & ए-: ०० \\
\hline MOGA & & 枸: : & \\
\hline MOEA/D-DRA & {$\left[\begin{array}{l}0-1 \\
\infty\end{array}\right.$} & $\forall-\infty$ & $\theta \cdots$ \\
\hline IBEA & F: & 秘 $\infty$ & $\square-\cdots$ \\
\hline HypE & E: & ⿶: & E:⿱一⿻上丨 \\
\hline \multirow[t]{2}{*}{ MO-CMA-ES } & ए: & 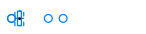 & Eio \\
\hline & WFG5.2.30 & WFG5.2.40 & WFG5.2.50 \\
\hline SPEA2 & D-100 & 淐 & 泟: 0 \\
\hline SMS-EMOA & $\forall \div$ & Bi⿻ & 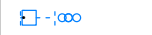 \\
\hline NSGA-III & : & E: & f:0000 \\
\hline NSGA-II & $\square^{-\cdots}$ & Ep & অळ \\
\hline MOGA & $\because ; \infty \infty$ & EH & : 000 \\
\hline MOEA/D-DRA & Eio & E: & \\
\hline IBEA & $\square-1 \infty$ & v:0 & $\forall--100$ \\
\hline HypE & E:0000 0 & घ: 。 & 沺 $\div 0000$ \\
\hline \multirow[t]{2}{*}{ MO-CMA-ES } & E:œ & E: & $\because ; \infty$ \\
\hline & WFG4.2.30 & WFG4.2.40 & WFG4.2.50 \\
\hline SPEA2 & F⿻ & Fioo & F。 \\
\hline SMS-EMOA & : & Fio & 畞: 。 \\
\hline NSGA-III & 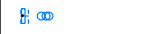 & : $-1, \infty \infty$ & Ei 0 \\
\hline NSGA-II & 讨 $০ 0 \oplus \infty$ & - 1000 & E \\
\hline MOGA & 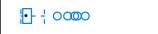 & HO & 방 \\
\hline MOEA/D-DRA & E:o & E: : 00 & itio \\
\hline IBEA & 沺: & 防 & Bp o \\
\hline HypE & $\forall-1,00$ & $\cdot-\cdots$ & 酒: 。 \\
\hline \multirow[t]{2}{*}{ MO-CMA-ES } & Hit 00000 & 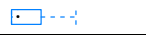 & 垱: \\
\hline & WFG3.2.30 & WFG3.2.40 & WFG3.2.50 \\
\hline SPEA2 & H' & $\square \cdots$ & Ep \\
\hline SMS-EMOA & E: & 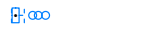 & Eio \\
\hline NSGA-III & 低 & Epo & Fi \\
\hline NSGA-II & gpo & $\square-1 \infty$ & $\square: 0 \quad 0$ \\
\hline MOGA & iG: & Hiono & 日。 \\
\hline MOEA/D-DRA & is: & $\forall ; \infty$ & E: \\
\hline IBEA & H & 啊-100 & 蛸 \\
\hline HypE & 酒: & Bico & H: \\
\hline MO-CMA-ES & E: & 近- & F: \\
\hline \multicolumn{3}{|c|}{0.00 .10 .20 .30 .4} & 0.00 .10 .2 \\
\hline
\end{tabular}

Figure 61: Boxplots of the $I_{H}^{r d}$ for $M=2$ problems, $n_{\mathbf{v a r}} \in\{30,40,50\}$ and $\boldsymbol{F E}_{\mathbf{m a x}}=40000$. 

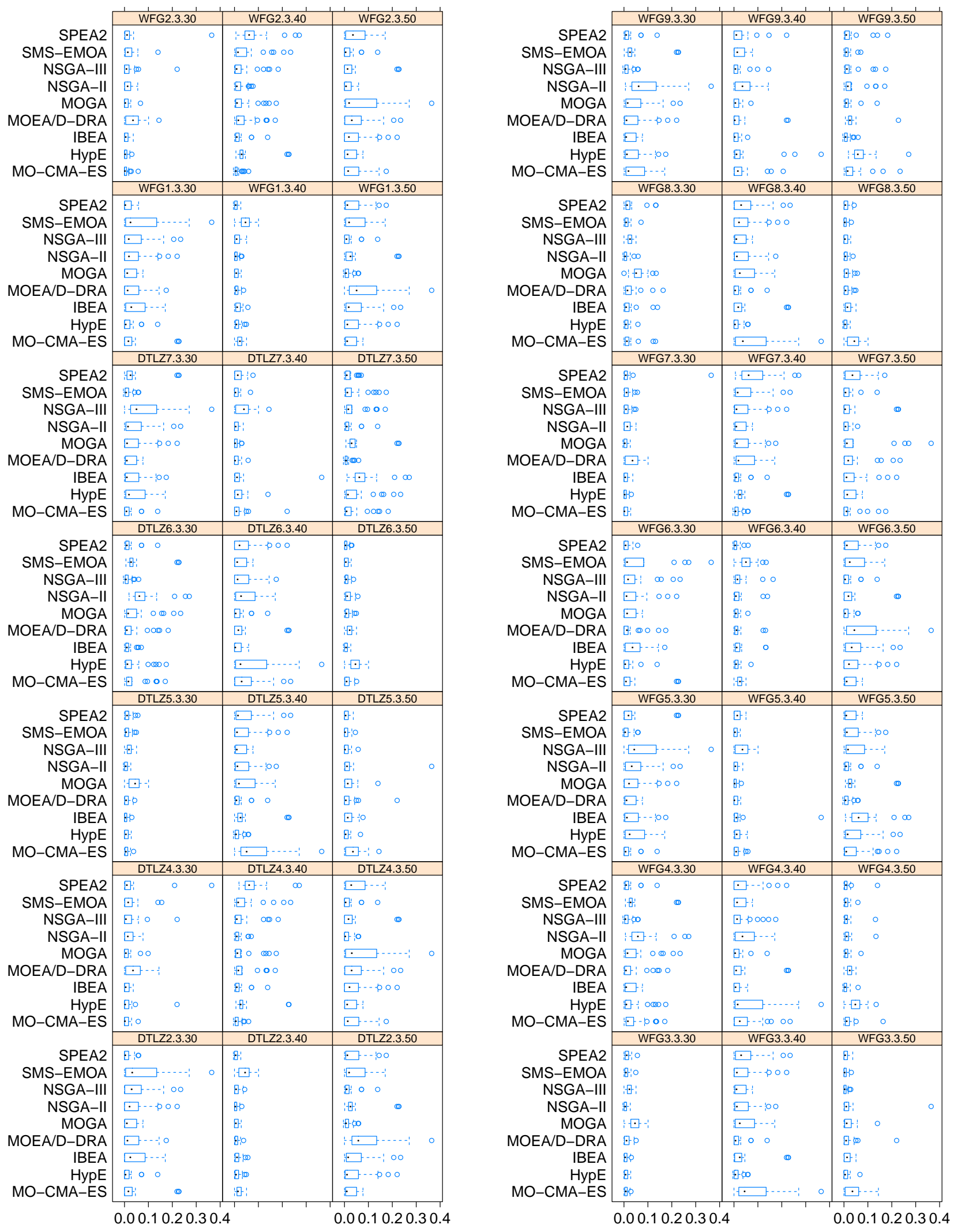

Figure 62: Boxplots of the $I_{H}^{\boldsymbol{r d}}$ for $M=3$ problems, $n_{\mathbf{v a r}} \in\{30,40,50\}$ and $\boldsymbol{F} \boldsymbol{E}_{\max }=40000$. 


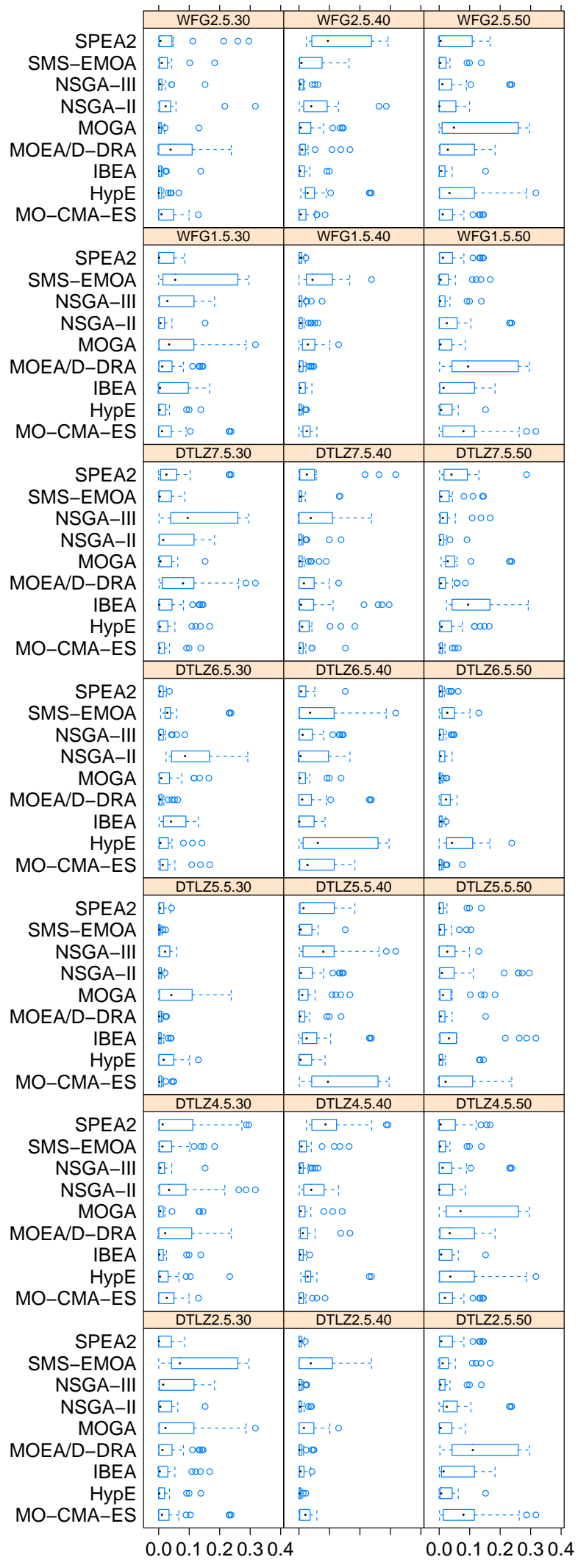

\begin{tabular}{|c|c|c|c|}
\hline \multirow{11}{*}{$\begin{array}{r}\text { SPEA2 } \\
\text { SMS-EMOA } \\
\text { NSGA-III } \\
\text { NSGA-II } \\
\text { MOGA } \\
\text { MOEA/D-DRA } \\
\text { IBEA } \\
\text { HypE } \\
\text { MO-CMA-ES }\end{array}$} & WFG9.5.30 & WFG9.5.40 & WFG9.5.50 \\
\hline & E: $\infty \circ$ & E: 0 & theos \\
\hline & 田- & $\square^{-\cdots}+\infty$ & $\square-\square$ \\
\hline & $\nabla-i$ & E:० & E: \\
\hline & - & 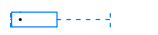 & 讨: 0 \\
\hline & 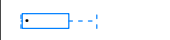 & E: $\infty \circ$ & \\
\hline & घ: 0 & $\nabla-\div 0$ & 畞: \\
\hline & : & $\cdot-\cdot$ & EHo \\
\hline & $\square \div \infty$ & $r^{-\cdots}$ & $: \cdot[-\cdots$ \\
\hline & 证: & $\square-\cdots$ & $F: 0 \circ \circ$ \\
\hline & WFG8.5.30 & WFG8.5.40 & WFG8.5.50 \\
\hline \multirow{10}{*}{$\begin{array}{r}\text { SPEA2 } \\
\text { SMS-EMOA } \\
\text { NSGA-III } \\
\text { NSGA-II } \\
\text { MOGA } \\
\text { MOEA/D-DRA } \\
\text { IBEA } \\
\text { HypE } \\
\text { MO-CMA-ES }\end{array}$} & 虾: & $\square-i$ & fio \\
\hline & E: & ஏ: & fitø \\
\hline & it: & $\square-\infty$ & $\cdot-10$ \\
\hline & & $\nabla-\infty$ & \\
\hline & $1-[-\square-1 \quad 0$ & $\forall: \infty$ & Eb \\
\hline & F:o & $E: \infty 0$ & fop \\
\hline & & $+\div \infty$ & \\
\hline & 迥-io & 口-i & 畹 \\
\hline & Epo & 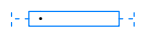 & $\cdot \cdot-\cdots$ \\
\hline & WFG7.5.30 & WFG7.5.40 & WFG7.5.50 \\
\hline \multirow{10}{*}{$\begin{array}{r}\text { SPEA2 } \\
\text { SMS-EMOA } \\
\text { NSGA-III } \\
\text { NSGA-II } \\
\text { MOGA } \\
\text { MOEA/D-DRA } \\
\text { IBEA } \\
\text { HypE } \\
\text { MO-CMA-ES }\end{array}$} & E00 & $\square$ & $\square-\cdots$ \\
\hline & E- & $\square^{--1}$ & $\theta: \infty \circ$ \\
\hline & & $\begin{array}{ll}-100 & 0\end{array}$ & V-io \\
\hline & ㄷ: & $: \cdot \cdot$ & $\cdot-\cdots$ \\
\hline & & $\nabla-1 \infty$ & 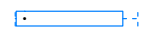 \\
\hline & $\cdot \cdot \cdots$ & 计: $\infty$ & $\cdot--1$ \\
\hline & & $E: \infty \circ$ & 日: 0 \\
\hline & & 计-1 & $\cdot \square-100$ \\
\hline & $\square-\uparrow 0$ & 曰-i & E:0000 \\
\hline & WFG6.5.30 & WFG6.5.40 & WFG6.5.50 \\
\hline \multirow{10}{*}{$\begin{array}{r}\text { SPEA2 } \\
\text { SMS-EMOA } \\
\text { NSGA-III } \\
\text { NSGA-II } \\
\text { MOGA } \\
\text { MOEA/D-DRA } \\
\text { IBEA } \\
\text { HypE } \\
\text { MO-CMA-ES }\end{array}$} & $\nabla-i$ & & $\nabla-\div \infty$ \\
\hline & $\cdot$ & $\therefore \cdot-\cdot 0$ & E: $\infty$ \\
\hline & $\left\ulcorner^{--1}\right.$ & E:O० & $F: \infty \circ$ \\
\hline & E: & & $\odot-\vdots 0$ \\
\hline & $\square-\cdots$ & :-io & \\
\hline & Fi: 000 & Eto & $1-r$ \\
\hline & $\square-\frac{1}{1}$ & & $\square^{--1}$ \\
\hline & $E: \infty$ & & ㅂ: 0 \\
\hline & F-10 0 & & 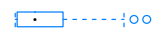 \\
\hline & WFG5.5.30 & WFG5.5.40 & WFG5.5.50 \\
\hline \multirow{10}{*}{$\begin{array}{r}\text { SPEA2 } \\
\text { SMS-EMOA } \\
\text { NSGA-III } \\
\text { NSGA-II } \\
\text { MOGA } \\
\text { MOEA/D-DRA } \\
\text { IBEA } \\
\text { HypE } \\
\text { MO-CMA-ES }\end{array}$} & $\begin{array}{rll}-10 & 0\end{array}$ & & $\because \cdot \square \div 00$ \\
\hline & $\nabla^{-1}$ & & $\nabla-\infty$ \\
\hline & $1-\square$ & 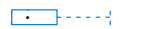 & F: \\
\hline & $\square^{--1}$ & & E: $\infty$ \\
\hline & घ: 0 & & ; - - : \\
\hline & $\square-1,00$ & $\nabla-\uparrow 0$ & $\square-i$ \\
\hline & $\nabla \div \infty$ & Eio & $\div \cdot$ \\
\hline & E: & B: $\quad 0$ & $\square-1$ \\
\hline & $E: \infty \circ$ & fpoo $\circ$ & 政协 \\
\hline & WFG4.5.30 & WFG4.5.40 & WFG4.5.50 \\
\hline \multirow{10}{*}{$\begin{array}{r}\text { SPEA2 } \\
\text { SMS-EMOA } \\
\text { NSGA-III } \\
\text { NSGA-II } \\
\text { MOGA } \\
\text { MOEA/D-DRA } \\
\text { IBEA } \\
\text { HypE } \\
\text { MO-CMA-ES }\end{array}$} & F: $\infty$ & E: $\quad \circ$ & \\
\hline & 田-: & $\square$ & - -10 \\
\hline & $\nabla-i$ & Fit 0000 & Fto 0 \\
\hline & $i^{-}-\frac{1}{1}$ & $\square^{-\frac{1}{1}}$ & \\
\hline & $\square--i$ & $E: \infty \circ$ & \\
\hline & & $\nabla-i 0$ & 田: \\
\hline & 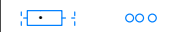 & $\square_{-i}^{-1}$ & \\
\hline & $\nabla \div \infty$ & $\cdot$ & $-\cdot \cdot-\frac{1}{1}$ \\
\hline & H & $\square^{--1}$ & F:०० \\
\hline & WFG3.5.30 & WFG3.5.40 & WFG3.5.50 \\
\hline \multirow{10}{*}{$\begin{array}{r}\text { SPEA2 } \\
\text { SMS-EMOA } \\
\text { NSGA-III } \\
\text { NSGA-II } \\
\text { MOGA } \\
\text { MOEA/D-DRA } \\
\text { IBEA } \\
\text { HypE } \\
\text { MO-CMA-ES }\end{array}$} & Fi: & $\square-1$ & \\
\hline & gh & $\square: 0$ & $f: \infty$ \\
\hline & 油 & {$[\cdot \cdots, 00$} & $\theta-10$ \\
\hline & Bi & $\nabla-\infty$ & F: 000 \\
\hline & 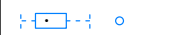 & E: $\infty$ & Fi: \\
\hline & fto 0 & F: $\infty \circ$ & H:O \\
\hline & 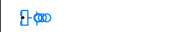 & $\cdot-10$ & $\nabla: \quad 0$ \\
\hline & - - io & $\nabla-i$ & \\
\hline & fitio & $\therefore \cdot$ & $\cdot \cdot^{-\cdots}$ \\
\hline & \multicolumn{2}{|c|}{0.00 .10 .20 .30 .4} & 0.00 .10 .20$. \\
\hline
\end{tabular}

Figure 63: Boxplots of the $I_{H}^{\boldsymbol{r d}}$ for $M=5$ problems, $n_{\mathrm{var}} \in\{30,40,50\}$ and $\boldsymbol{F} \boldsymbol{E}_{\max }=40000$. 

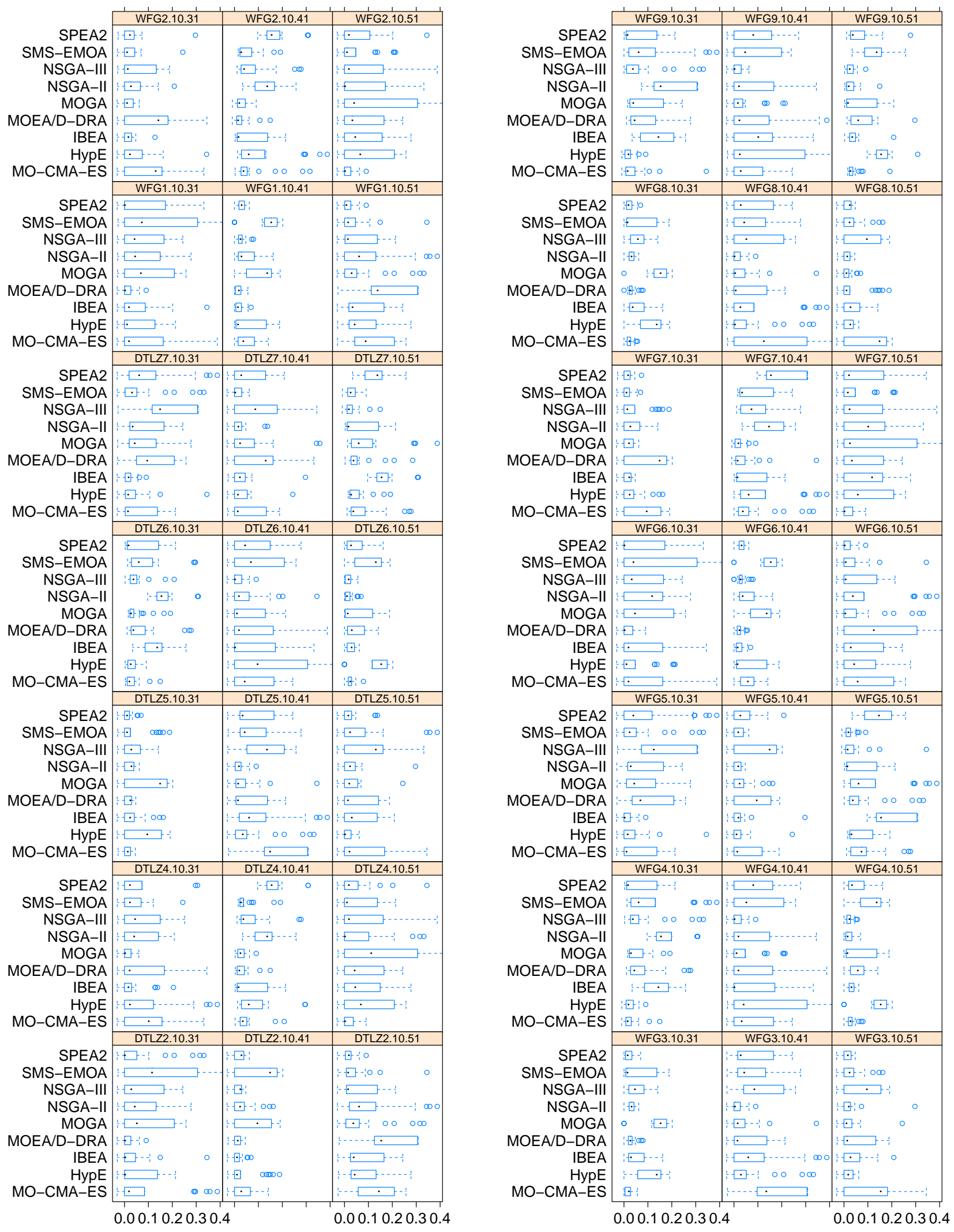

Figure 64: Boxplots of the $I_{H}^{\boldsymbol{r d}}$ for $M=10$ problems, $n_{\mathbf{v a r}} \in\{30,40,50\}$ and $\boldsymbol{F} \boldsymbol{E}_{\max }=40000$. 

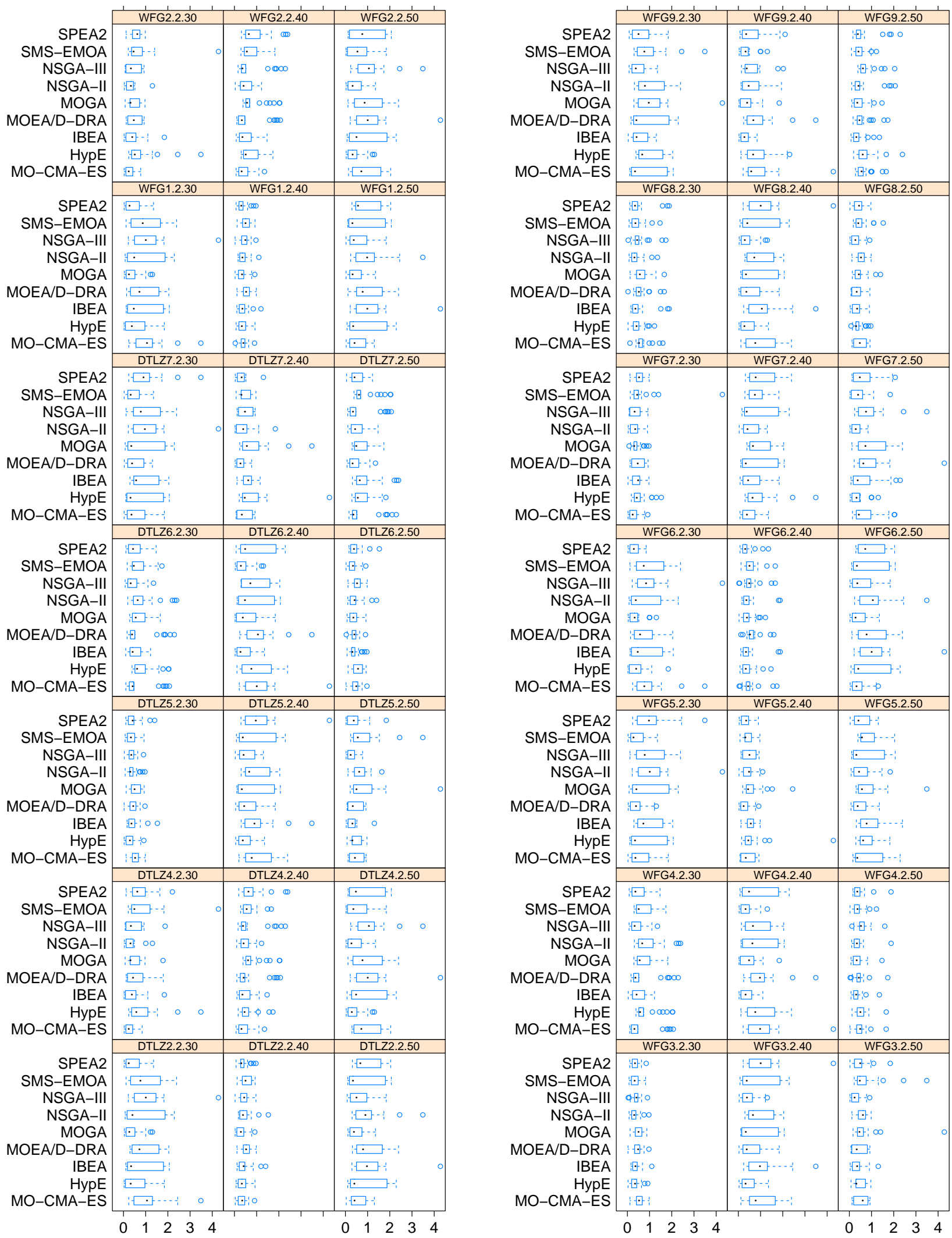

Figure 65: Boxplots of the $I_{\epsilon+}$ for $M=2$ problems, $n_{\mathbf{v a r}} \in\{30,40,50\}$ and $\boldsymbol{F E}_{\max }=2500$. 

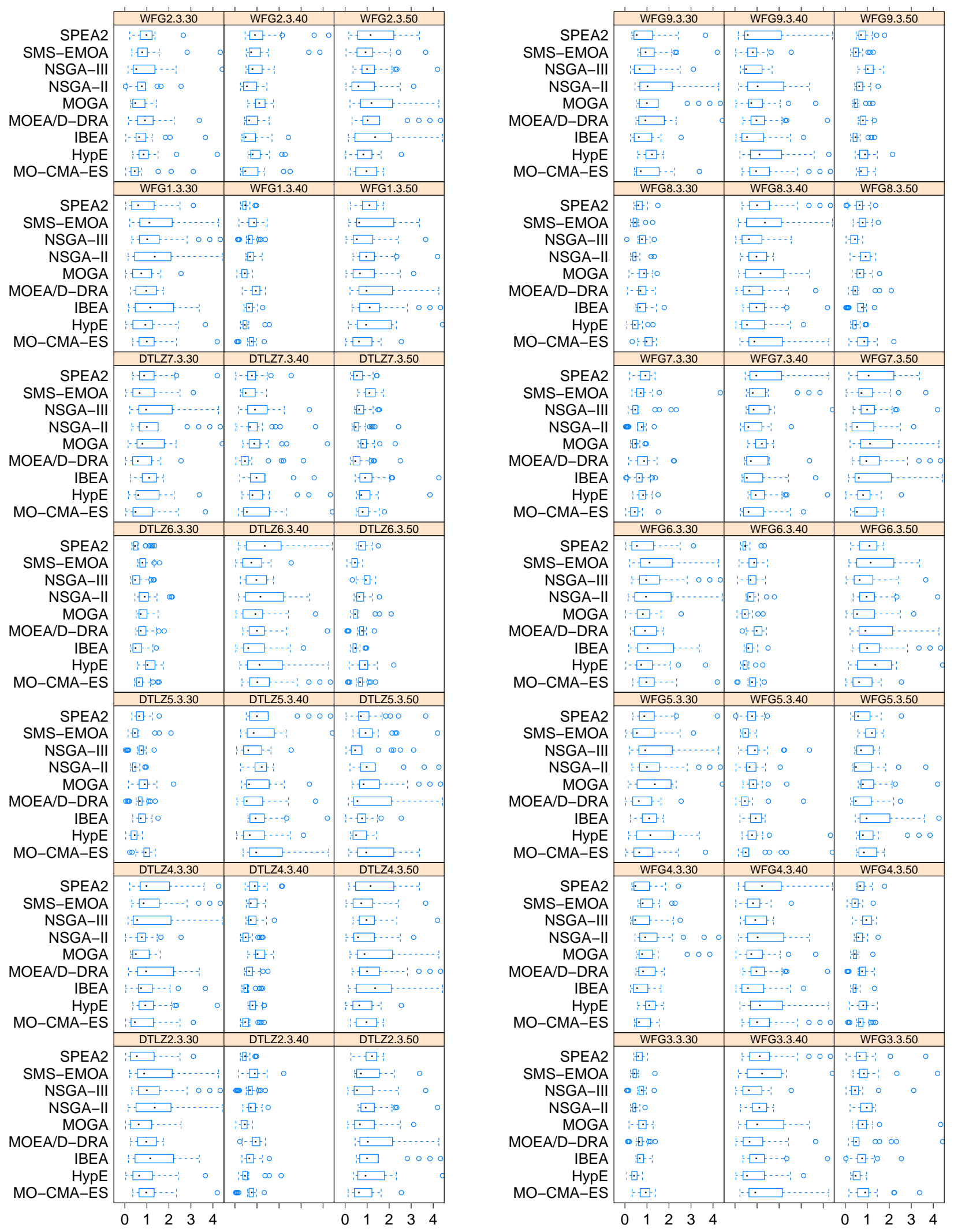

Figure 66: Boxplots of the $I_{\epsilon+}$ for $M=3$ problems, $n_{\mathbf{v a r}} \in\{30,40,50\}$ and $\boldsymbol{F} E_{\max }=2500$. 

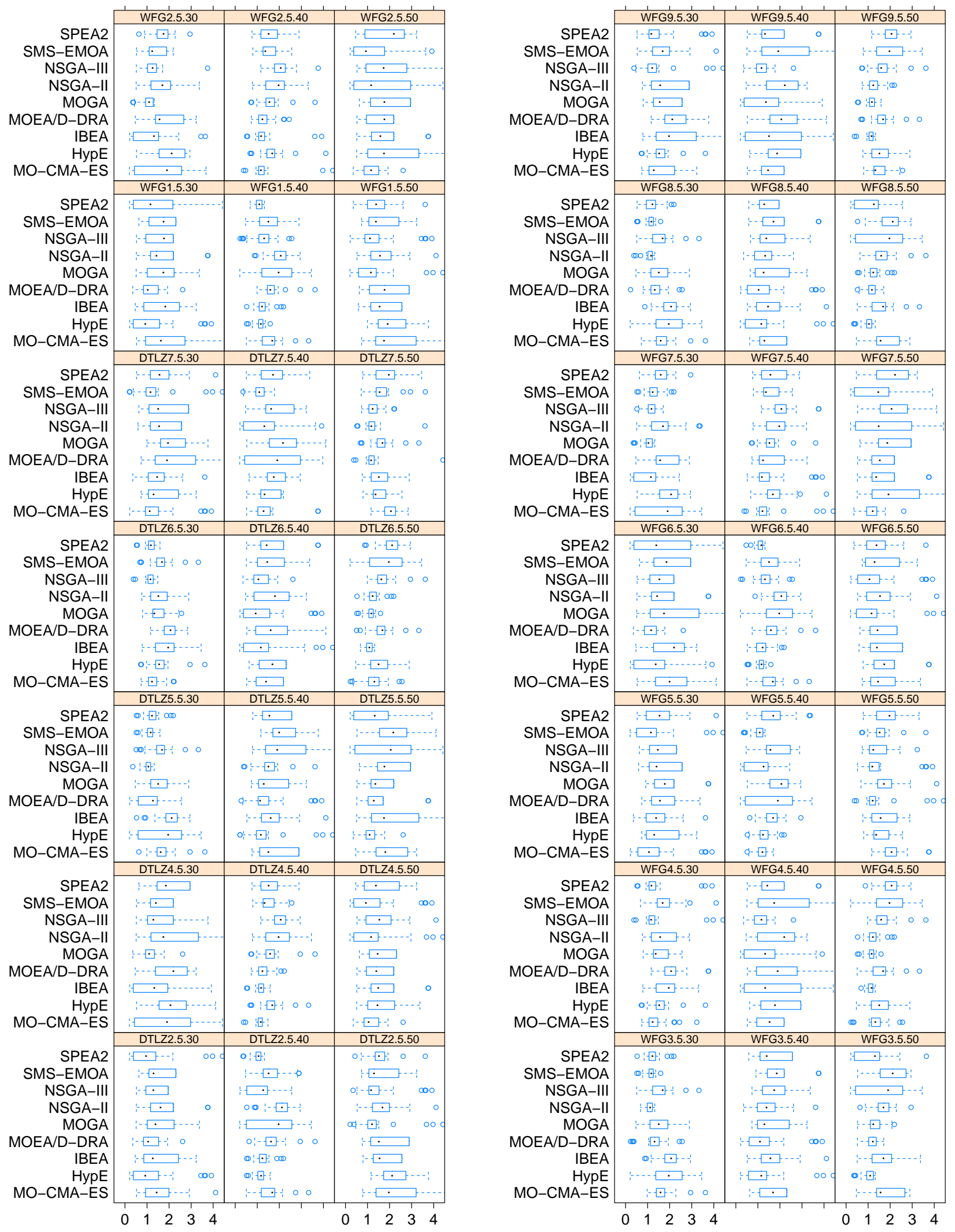

Figure 67: Boxplots of the $I_{\epsilon+}$ for $M=5$ problems, $n_{\mathbf{v a r}} \in\{30,40,50\}$ and $\boldsymbol{F} E_{\max }=2500$. 

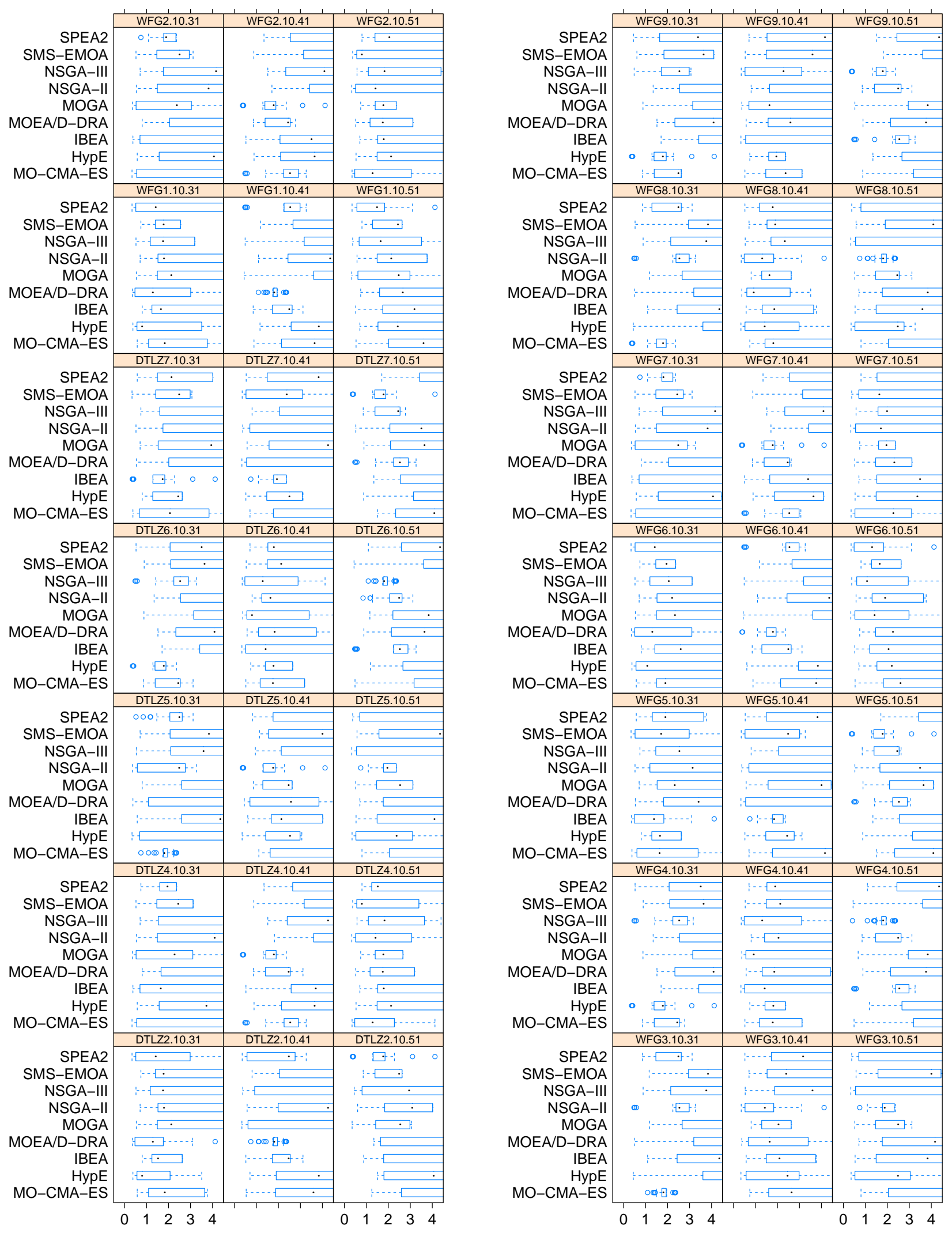

Figure 68: Boxplots of the $I_{\epsilon+}$ for $M=10$ problems, $n_{\mathbf{v a r}} \in\{30,40,50\}$ and $\boldsymbol{F E}_{\max }=2500$. 

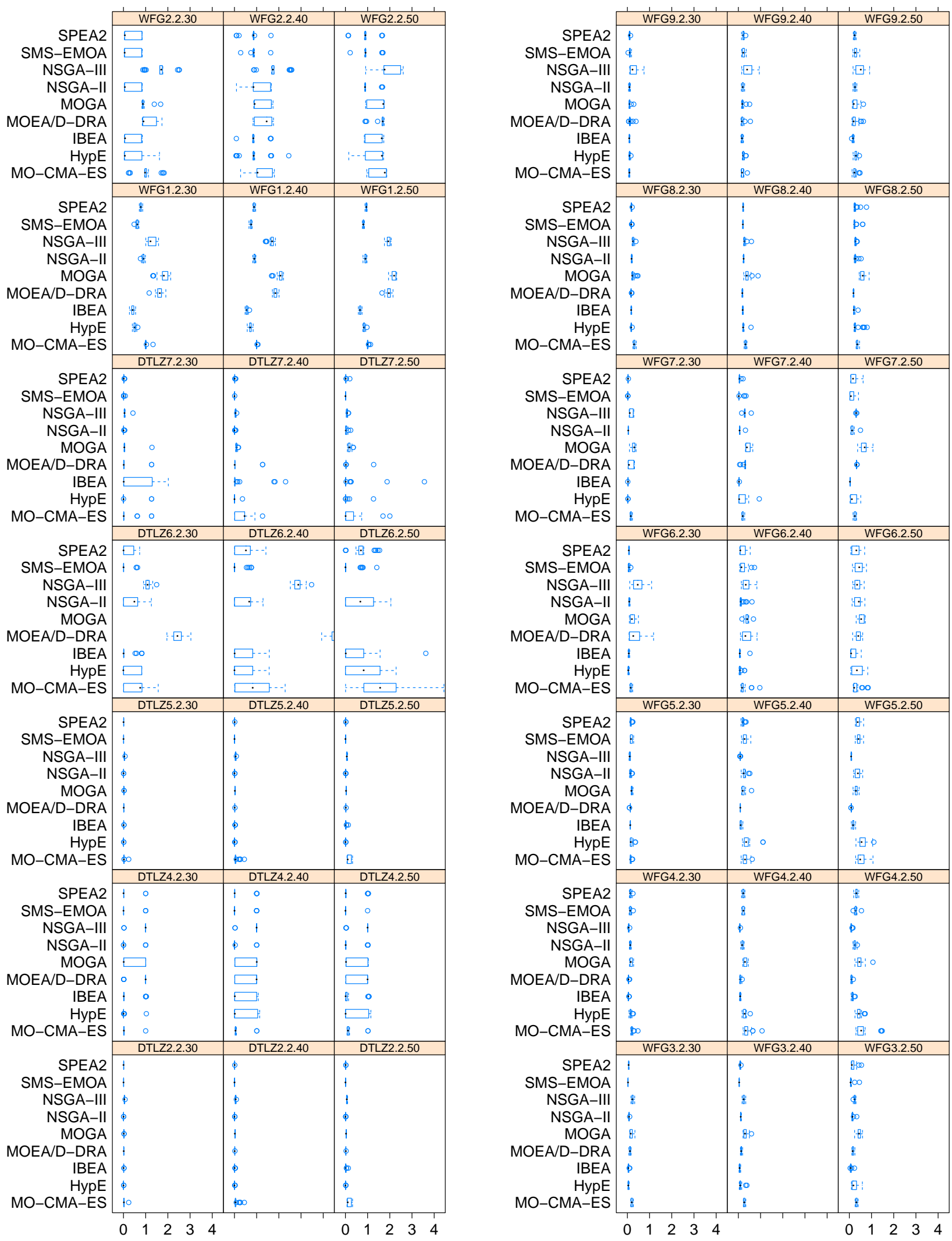

Figure 69: Boxplots of the $I_{\epsilon+}$ for $M=2$ problems, $n_{\mathbf{v a r}} \in\{30,40,50\}$ and $\boldsymbol{F E}_{\mathbf{m a x}}=10000$. 

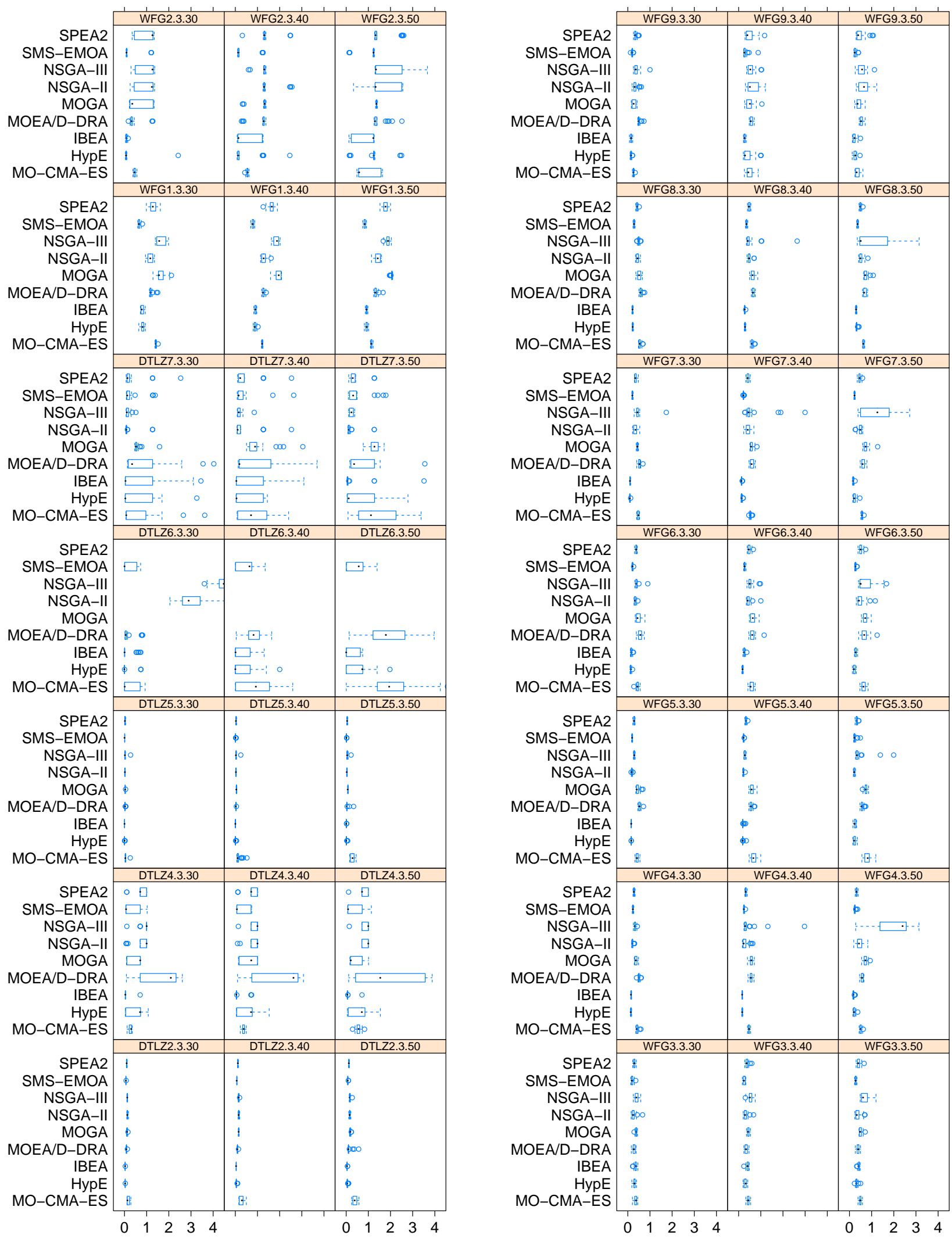

Figure 70: Boxplots of the $I_{\epsilon+}$ for $M=3$ problems, $n_{\mathbf{v a r}} \in\{30,40,50\}$ and $\boldsymbol{F} \boldsymbol{E}_{\max }=10000$. 

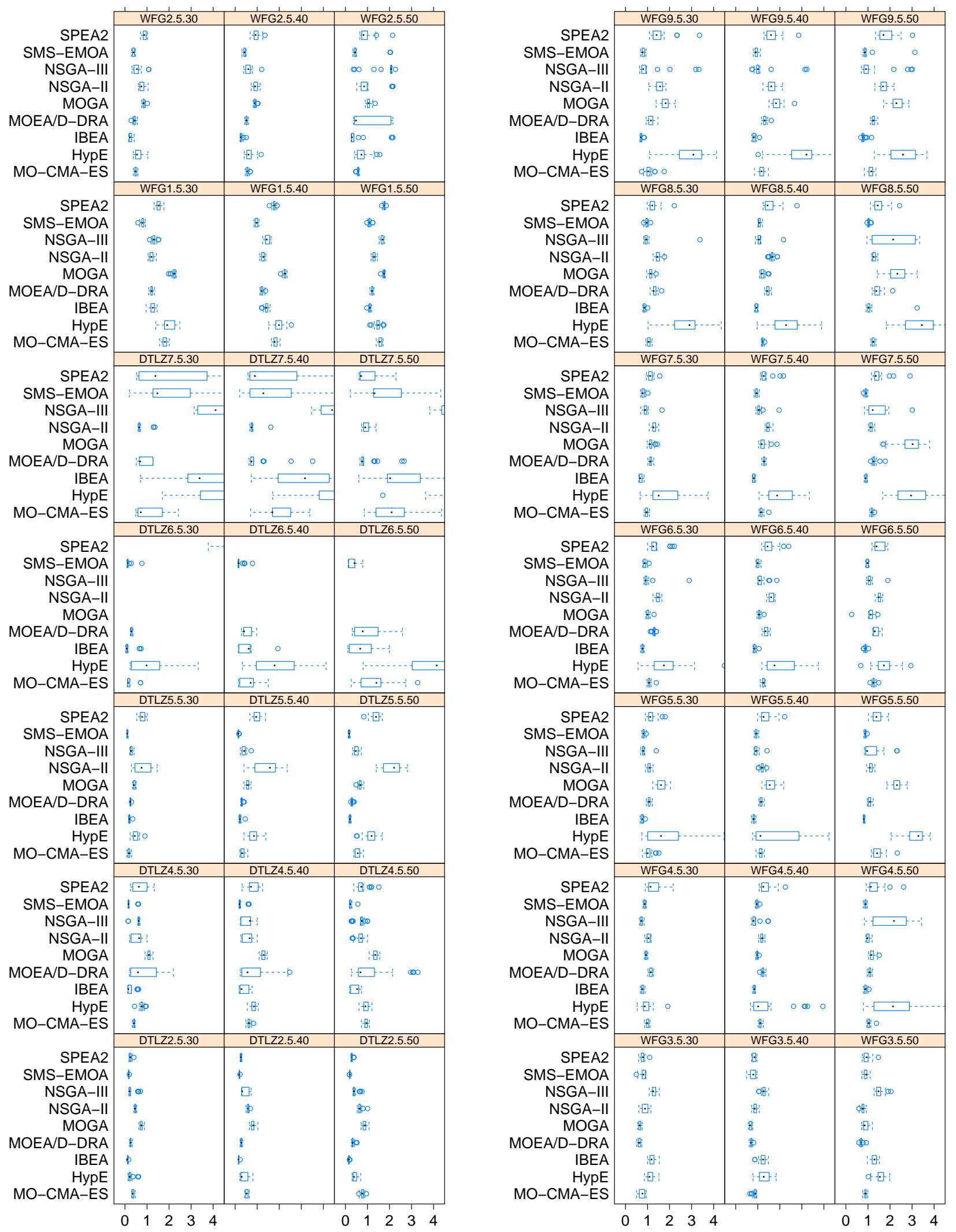

Figure 71: Boxplots of the $I_{\epsilon+}$ for $M=5$ problems, $n_{\mathbf{v a r}} \in\{30,40,50\}$ and $\boldsymbol{F E}_{\max }=10000$. 

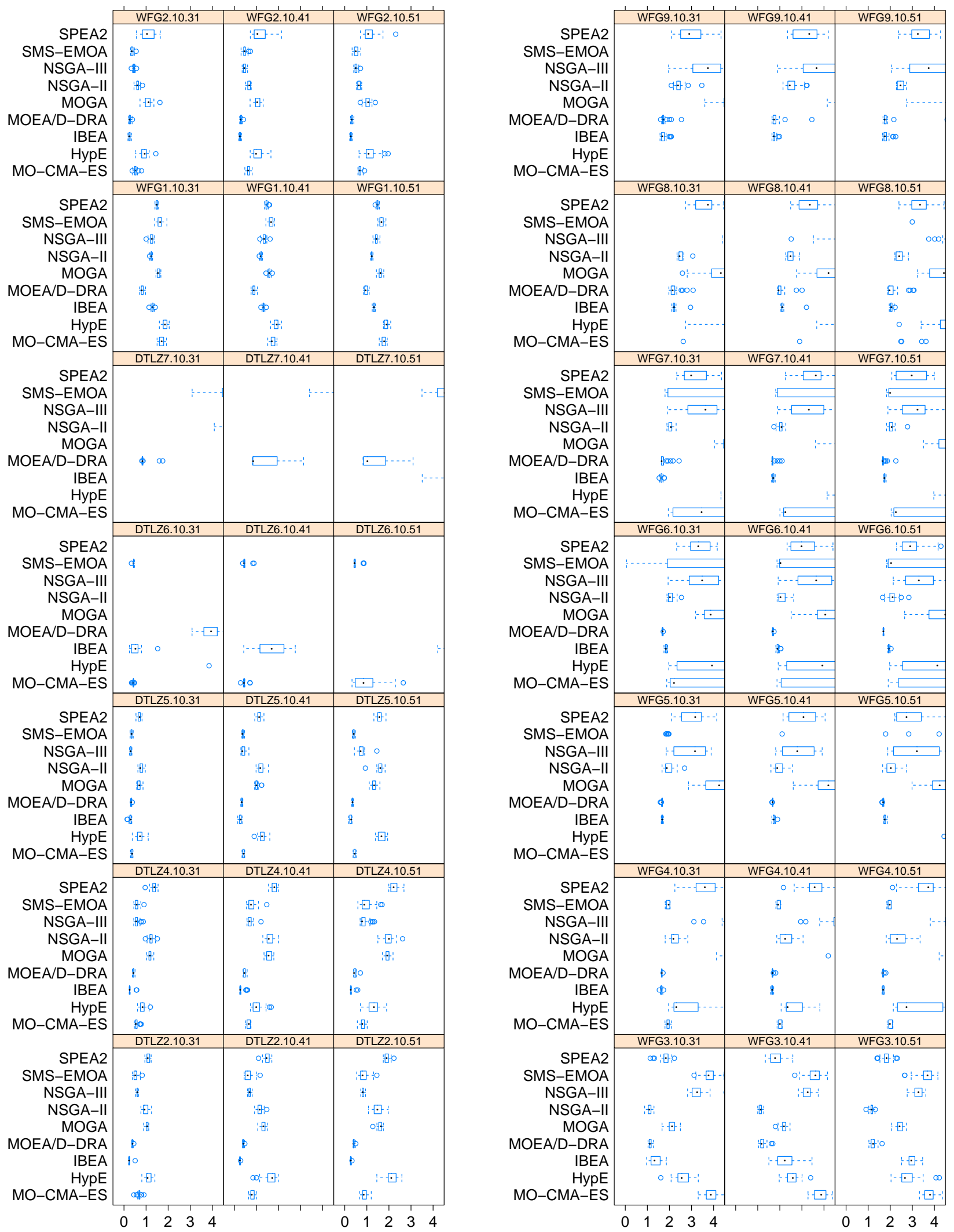

Figure 72: Boxplots of the $I_{\epsilon+}$ for $M=10$ problems, $n_{\mathbf{v a r}} \in\{30,40,50\}$ and $\boldsymbol{F E}_{\mathbf{m a x}}=10000$. 


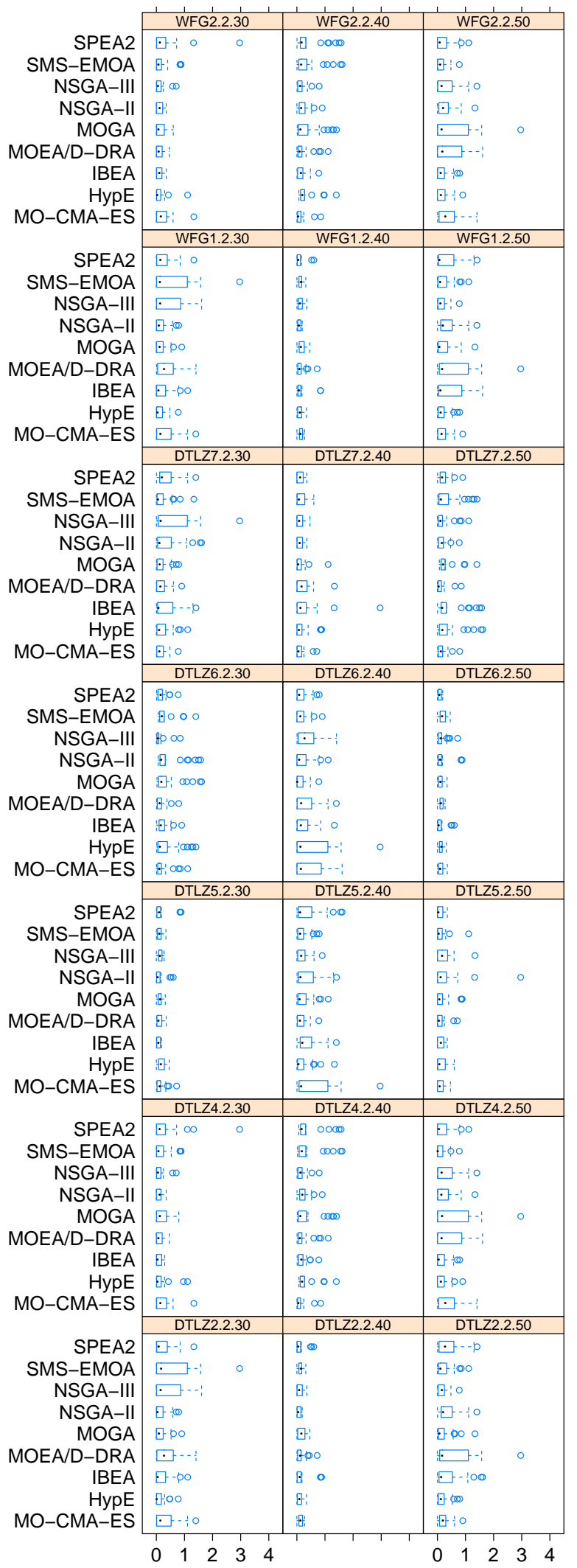

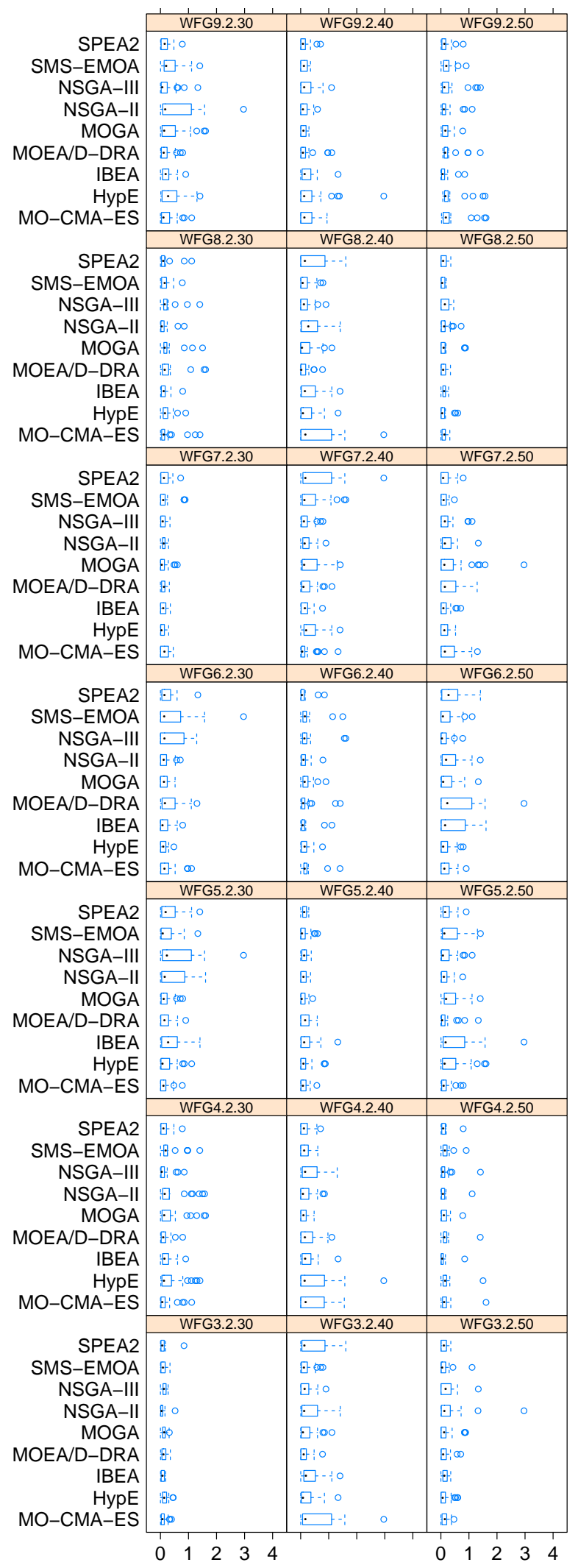

Figure 73: Boxplots of the $I_{\epsilon+}$ for $M=2$ problems, $n_{\mathbf{v a r}} \in\{30,40,50\}$ and $\boldsymbol{F E}_{\max }=40000$. 

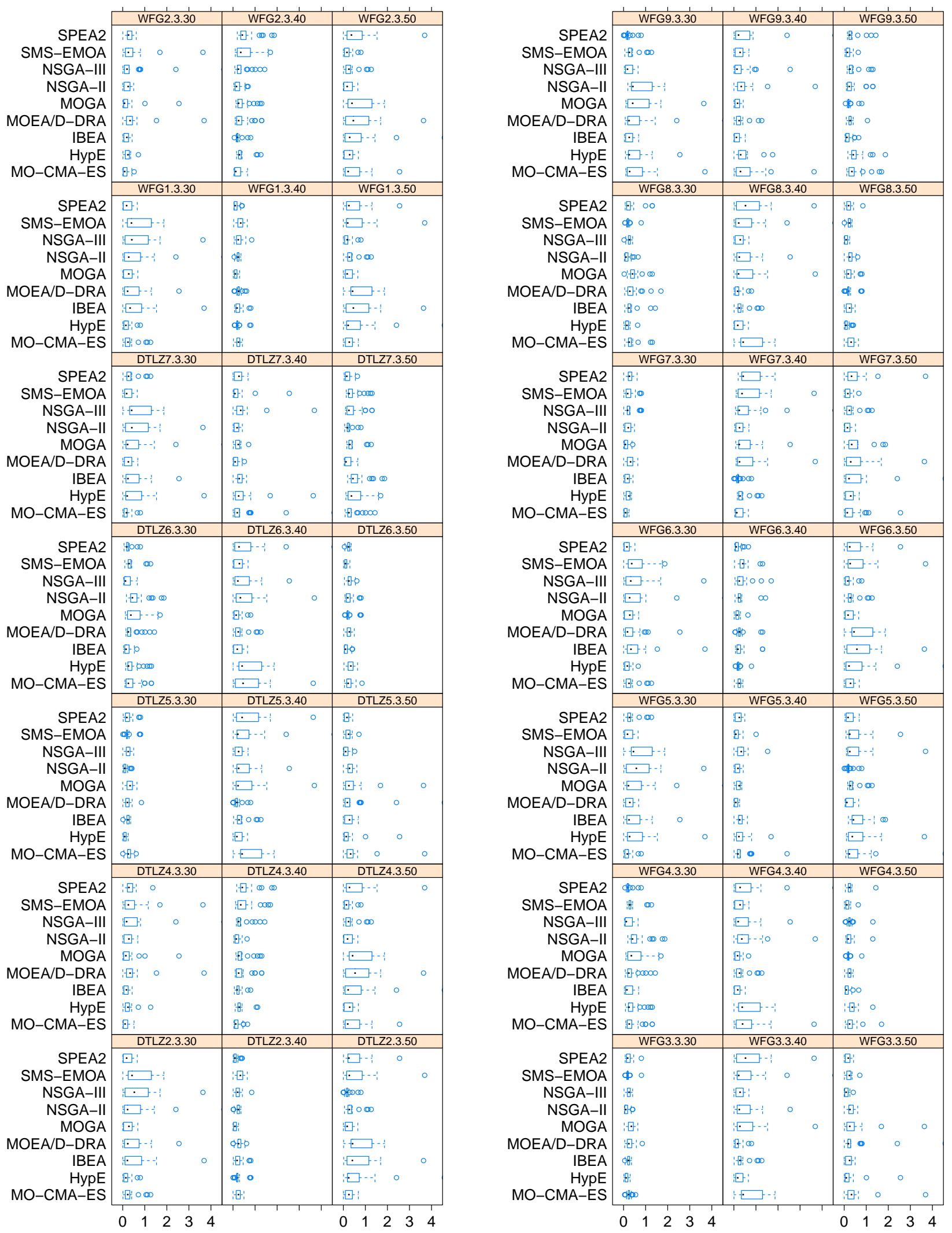

Figure 74: Boxplots of the $I_{\epsilon+}$ for $M=3$ problems, $n_{\mathbf{v a r}} \in\{30,40,50\}$ and $\boldsymbol{F E}_{\max }=40000$. 

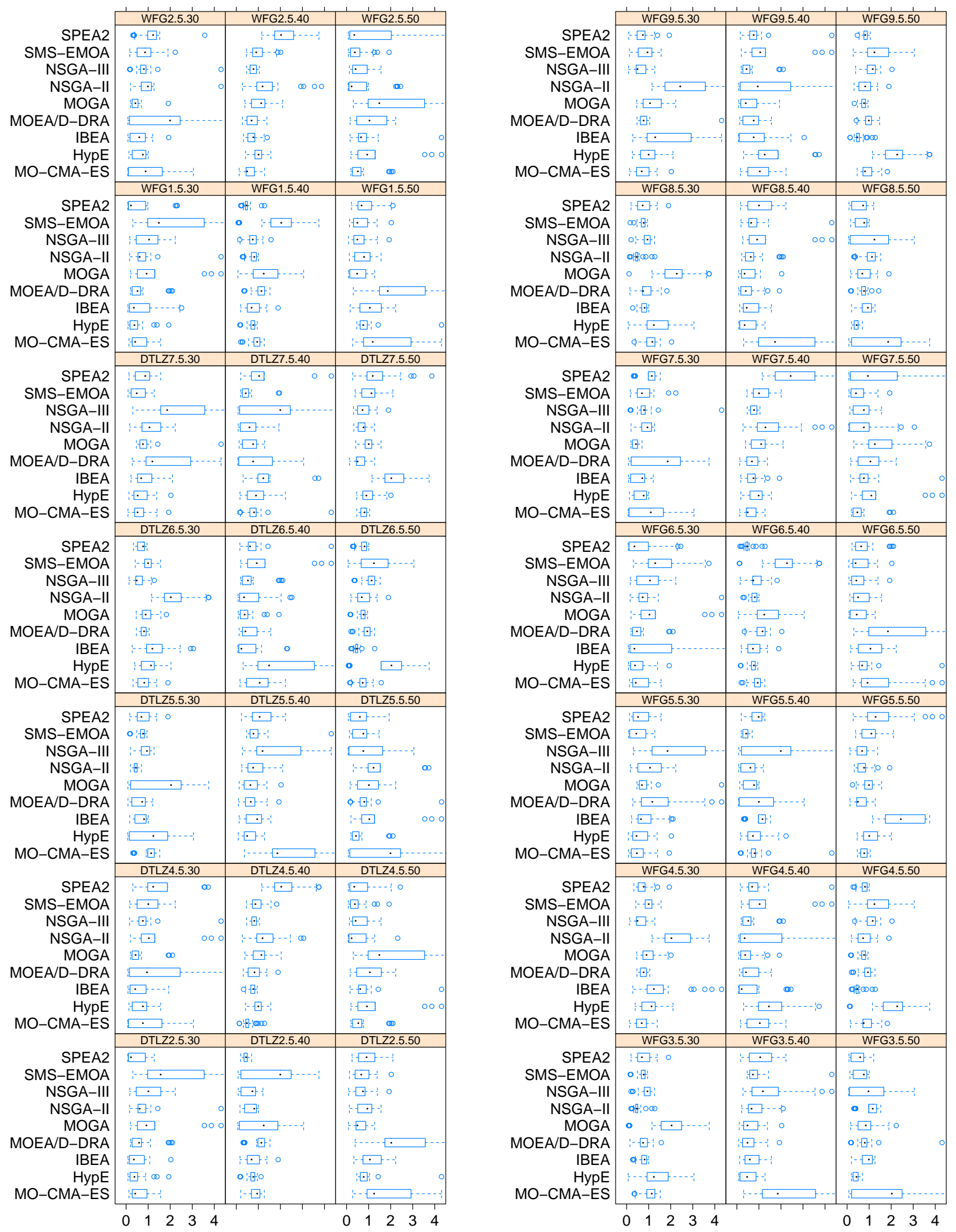

Figure 75: Boxplots of the $I_{\epsilon+}$ for $M=5$ problems, $n_{\mathbf{v a r}} \in\{30,40,50\}$ and $\boldsymbol{F E}_{\max }=40000$. 

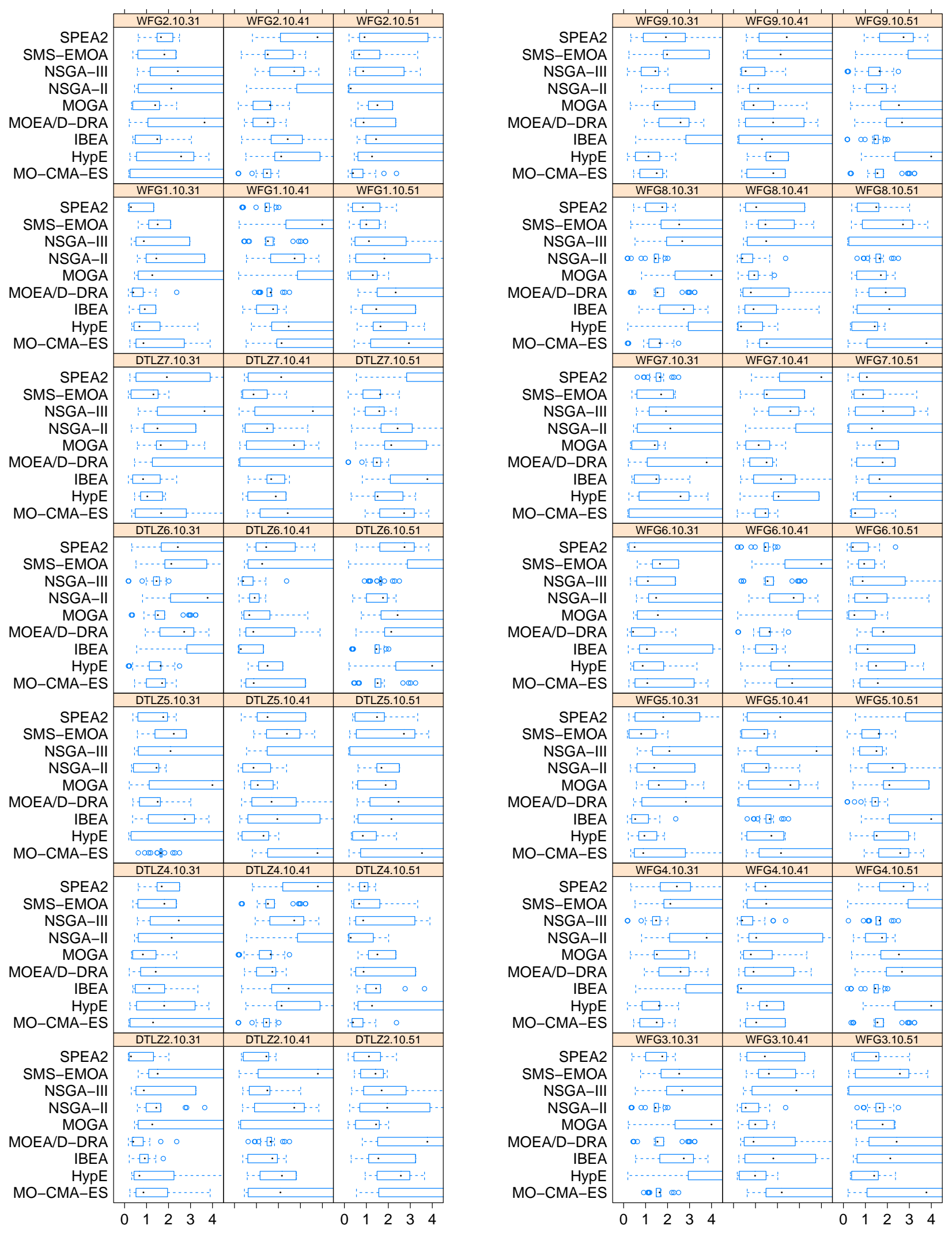

Figure 76: Boxplots of the $I_{\epsilon+}$ for $M=10$ problems, $n_{\mathbf{v a r}} \in\{30,40,50\}$ and $\boldsymbol{F E}_{\max }=40000$. 


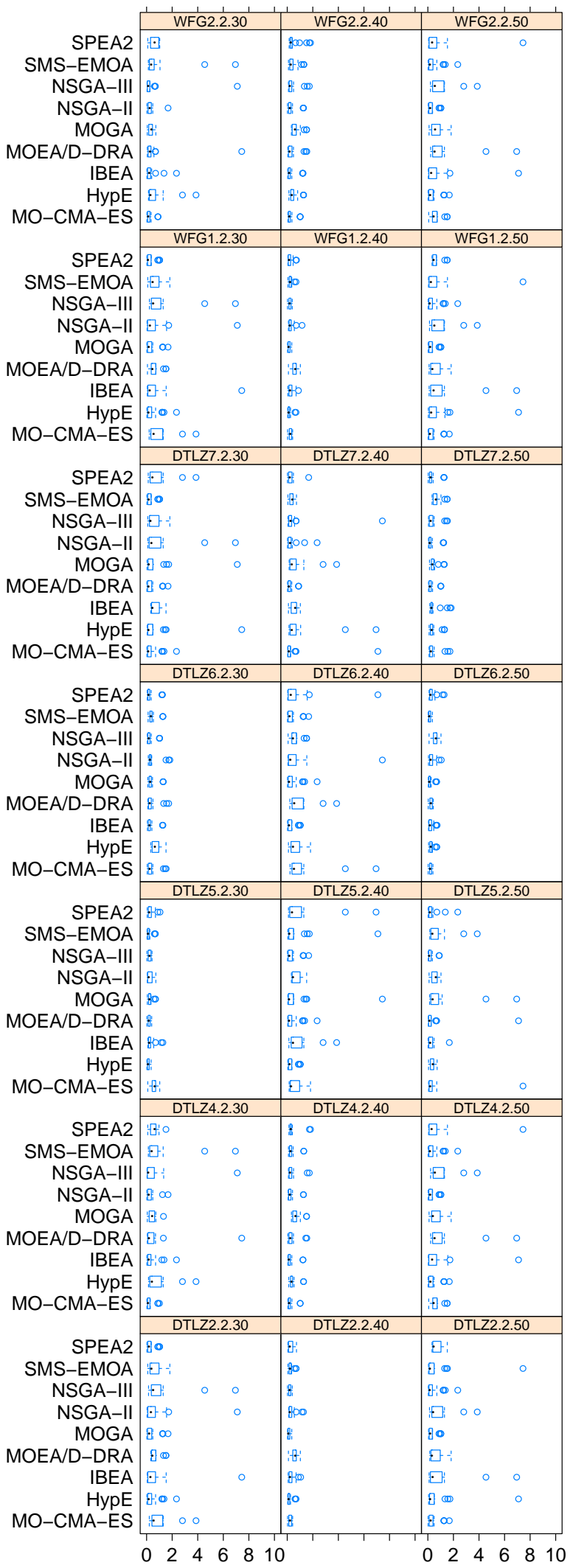

\begin{tabular}{|c|c|c|c|c|c|}
\hline & WFG9.2.30 & \multicolumn{2}{|c|}{ WFG9.2.40 } & \multicolumn{2}{|l|}{ WFG9.2.50 } \\
\hline SPEA2 & fi: & E. & $\circ$ & \multicolumn{2}{|l|}{ fto } \\
\hline SMS-EMOA & 实: 00 & $8 \infty$ & & tio 0 & \\
\hline NSGA-III & 80 & 怔p & & Hio & \\
\hline NSGA-II & $\theta \div$ & 口十 & $\circ$ & gi。 & \\
\hline MOGA & H & Eি० & & Hito & \\
\hline MOEA/D-DRA & $\theta$ a d & $\nabla: 0 \circ$ & & 8: 0 & \\
\hline IBEA & $\forall \rightarrow \infty$ & 80 & & fio & \\
\hline HypE & F: & 汇 $\div$ & & 10 & \\
\hline \multirow[t]{2}{*}{ MO-CMA-ES } & Ө由 & 阿: & $\circ$ & ifo & \\
\hline & WFG8.2.30 & \multicolumn{2}{|c|}{ WFG8.2.40 } & WFG8.2.50 & \\
\hline SPEA2 & 䍕 & \multicolumn{2}{|c|}{ Fis 0} & & \\
\hline SMS-EMOA & : & F舟 & 。 & fipo & \\
\hline NSGA-III & 采 & 田 $\infty$ & & fi & \\
\hline NSGA-II & H & 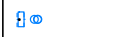 & & If: & \\
\hline MOGA & it & 분 & $\circ$ & fto & \\
\hline MOEA/D-DRA & if & Eio & & fo & \\
\hline IBEA & \&i & 沺 00 & & 目 & \\
\hline НypE & fi & g० & & B: & \\
\hline MO-CMA-ES & 湴: & 证 $\div$ & & \&p & \\
\hline & \begin{tabular}{|l} 
WFG7.2.30 \\
\end{tabular} & WFG7.2. & & WFG7.2.50 & \\
\hline SPEA2 & FG & 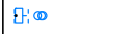 & & 田少 & $\circ$ \\
\hline SMS-EMOA & fto & E: & 。 & fi: & \\
\hline NSGA-III & to & $B \oplus$ & & 田 & \\
\hline NSGA-II & fi & $8 \infty$ & & 80 & \\
\hline MOGA & E: & ⿶: & & 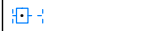 & \\
\hline MOEA/D-DRA & go & $8 \oplus$ & & : $\quad 0$ & b \\
\hline IBEA & fio & B๑० & & & 。 \\
\hline HypE & 渭。 & 路: & & $8 \infty$ & \\
\hline MO-CMA-ES & $\mathbb{F}_{i}$ & fo & & 细p & \\
\hline & \begin{tabular}{|l} 
WFG6.2.30 \\
\end{tabular} & WFG6.2. & & WFG6.2.50 & \\
\hline SPEA2 & 80 & $\mathrm{BH}_{\mathrm{H}}$ & & ย๑ & \\
\hline SMS-EMOA & 壮-1 & A & & E+ & ○ \\
\hline NSGA-III & 词 & 齿 & & fio & \\
\hline NSGA-II & E-p & B: & & 回 00 & \\
\hline MOGA & Ei $\infty$ & \&i: & & fo & \\
\hline MOEA/D-DRA & 证p & it: & & 证- & \\
\hline IBEA & $\square \div$ & $8:$ & & 回: & o \\
\hline HypE & f:०० & \# & & 河如 & \\
\hline MO-CMA-ES & 回 $0 \circ$ & i & & $8 \infty$ & \\
\hline & \begin{tabular}{|l} 
WFG5.2.30 \\
\end{tabular} & WFG5.2. & & WFG5.2.50 & \\
\hline SPEA2 & 洄 ○。 & 采 & & $8 \infty$ & \\
\hline SMS-EMOA & 80 & E: & & F: & \\
\hline NSGA-III & $0-1$ & Bp & $\circ$ & $8 \oplus$ & \\
\hline NSGA-II & 吅 & $: 900$ & & \&i 00 & \\
\hline MOGA & F协 & Ei० & & Hio 0 & \\
\hline MOEA/D-DRA & $8 \infty$ & \&io & & 80 & \\
\hline IBEA & Ө® & 渵 & & Fifiod & \\
\hline HypE & 분 & Ep & $\circ$ & 踝 & \\
\hline MO-CMA-ES & घंの० & Bo & 。 & $8 \oplus$ & \\
\hline & \begin{tabular}{|l} 
WFG4.2.30 \\
\end{tabular} & WFG4.2. & & WFG4.2.50 & \\
\hline SPEA2 & \#10 & F- & $\circ$ & Hio & \\
\hline SMS-EMOA & 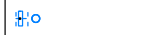 & $8 \infty$ & & 8: & \\
\hline NSGA-III & Hi० & 闰必 & & it: & \\
\hline NSGA-II & fpocos & 口: & $\circ$ & gi: & \\
\hline MOGA & 㖪 & Ë० & & H & \\
\hline MOEA/D-DRA & fi: $\infty$ & : & & 要 & \\
\hline IBEA & $\frac{81}{4} \circ$ & 80 & & 8: & \\
\hline HypE & Bi $\infty$ & 细: & & $\mathscr{2}$ & \\
\hline MO-CMA-ES & E & 田: $\quad \circ$ & $\circ$ & 㐘 & \\
\hline & \begin{tabular}{|l} 
WFG3.2.30 \\
\end{tabular} & WFG3.2. & & WFG3.2.50 & \\
\hline SPEA2 & 敤 & 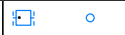 & $\circ$ & Roo & \\
\hline SMS-EMOA & po & 国如 & 。 & E: 00 & \\
\hline NSGA-III & 㐘 & $\theta \infty$ & & fio & \\
\hline NSGA-II & fio & 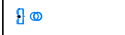 & & 润 & \\
\hline MOGA & tho & E: & $\circ$ & Do & \\
\hline MOEA/D-DRA & 米 & B:० & & fo & p \\
\hline IBEA & pso & 沺 00 & & 思。 & \\
\hline HypE & $F_{i}$ & fo & & Fi: & \\
\hline MO-CMA-ES & 洁 & 计： & & gp & $\circ$ \\
\hline
\end{tabular}

Figure 77: Boxplots of the $I_{I G D}$ for $M=2$ problems, $n_{\mathbf{v a r}} \in\{30,40,50\}$ and $\boldsymbol{F E}_{\max }=2500$. 

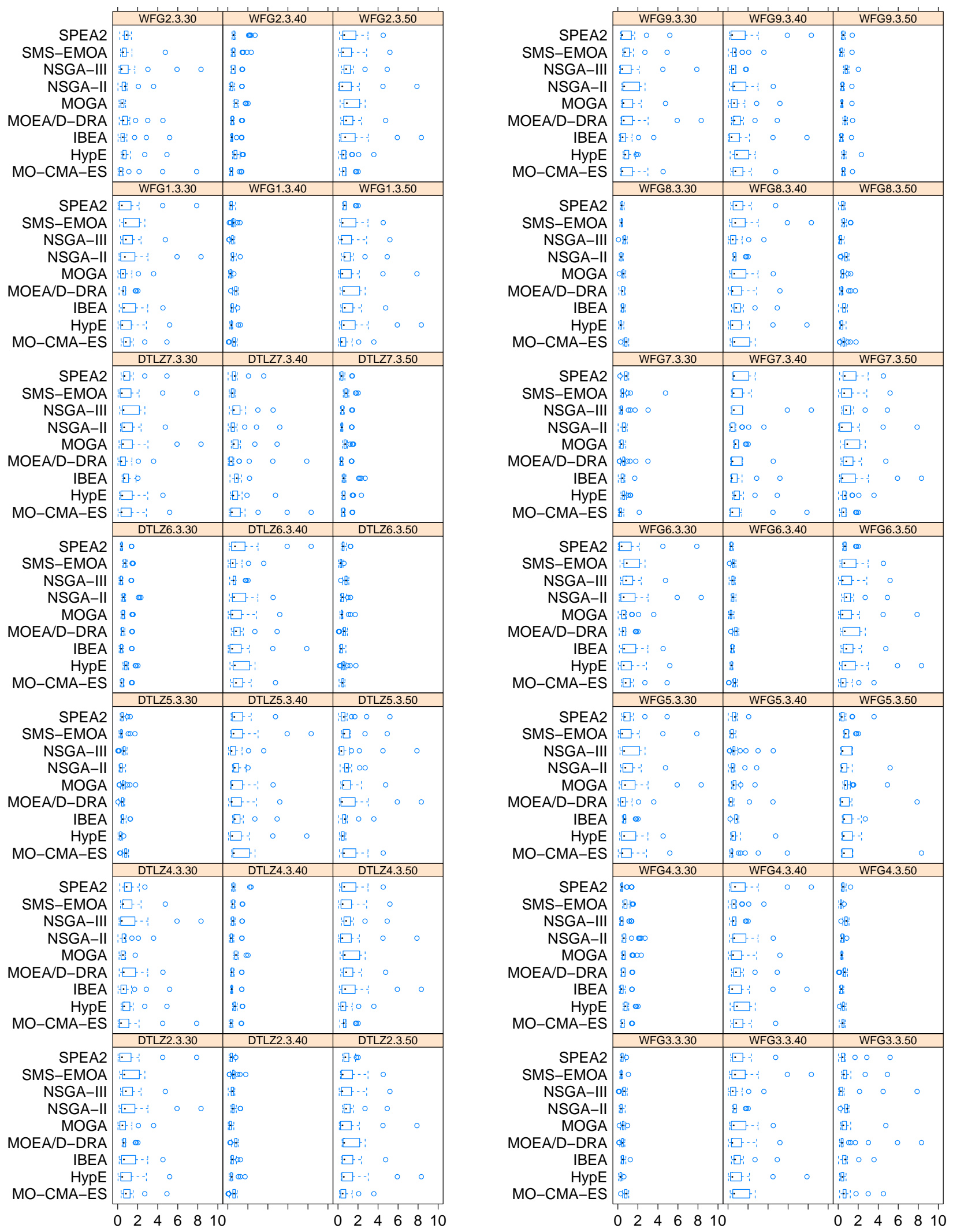

Figure 78: Boxplots of the $I_{I G D}$ for $M=3$ problems, $n_{\mathrm{var}} \in\{30,40,50\}$ and $\boldsymbol{F E}_{\max }=2500$. 

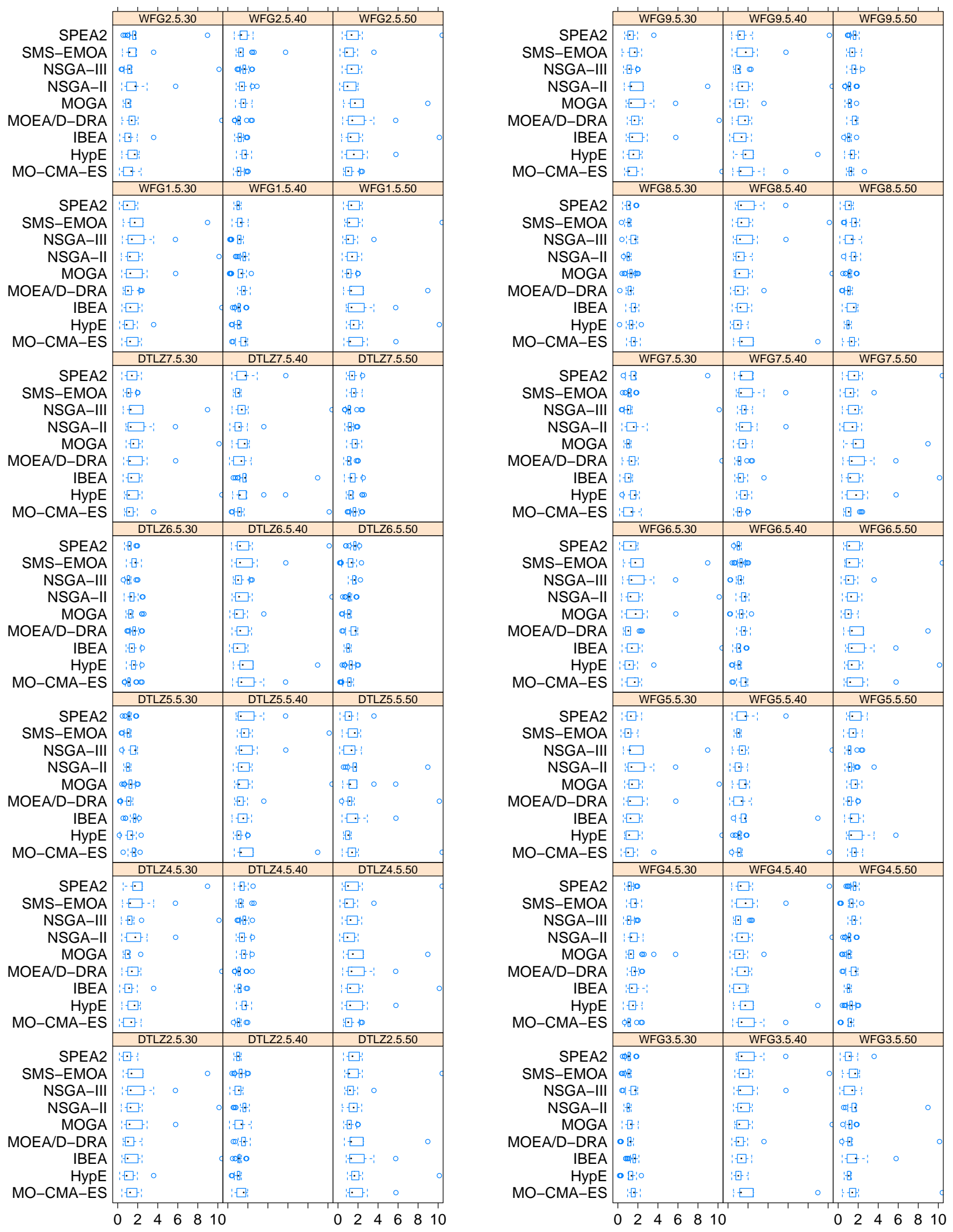

Figure 79: Boxplots of the $I_{I G D}$ for $M=5$ problems, $n_{\mathbf{v a r}} \in\{30,40,50\}$ and $\boldsymbol{F E}_{\max }=2500$. 

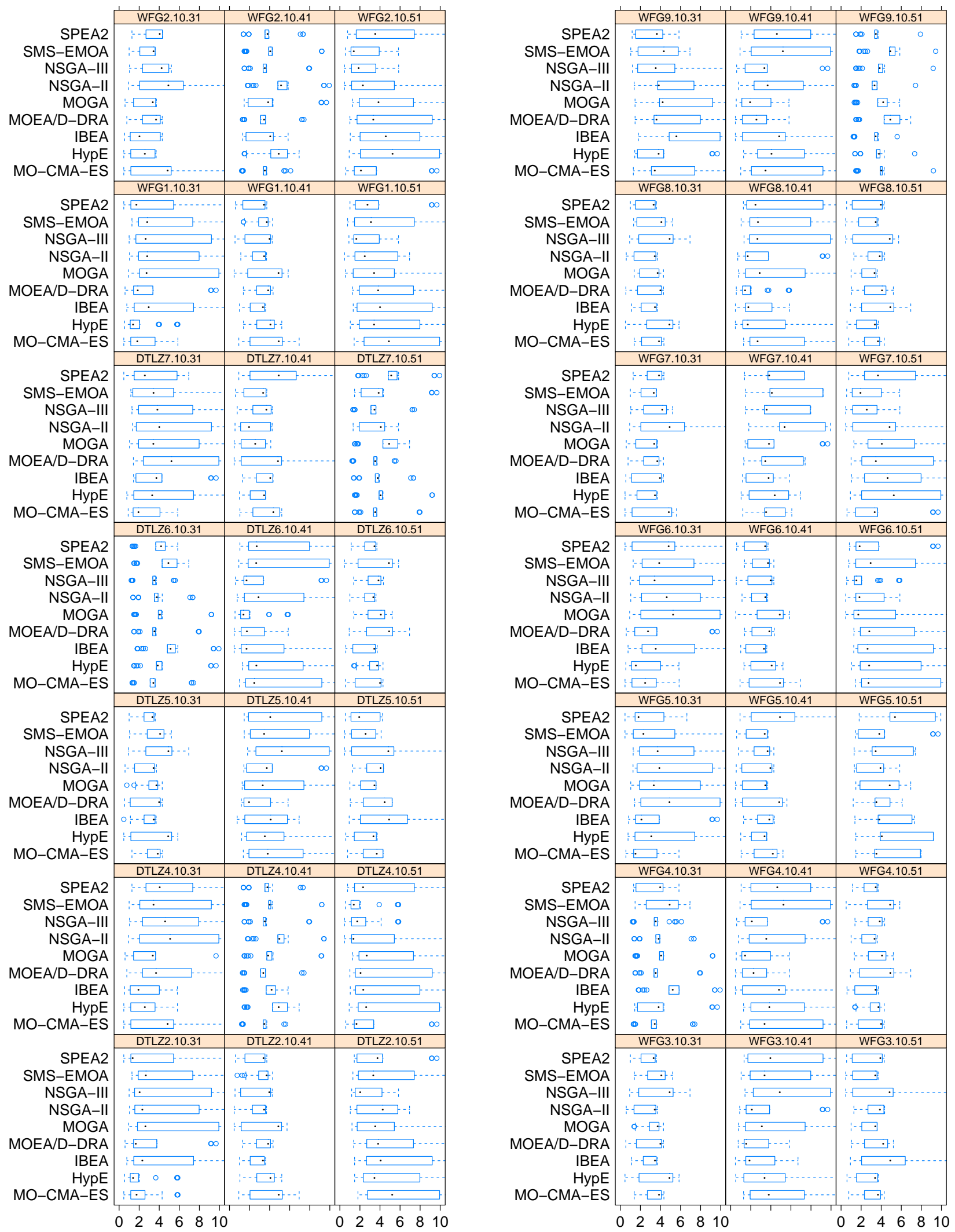

Figure 80: Boxplots of the $I_{I G D}$ for $M=10$ problems, $n_{\mathbf{v a r}} \in\{30,40,50\}$ and $\boldsymbol{F E}_{\mathbf{m a x}}=2500$. 

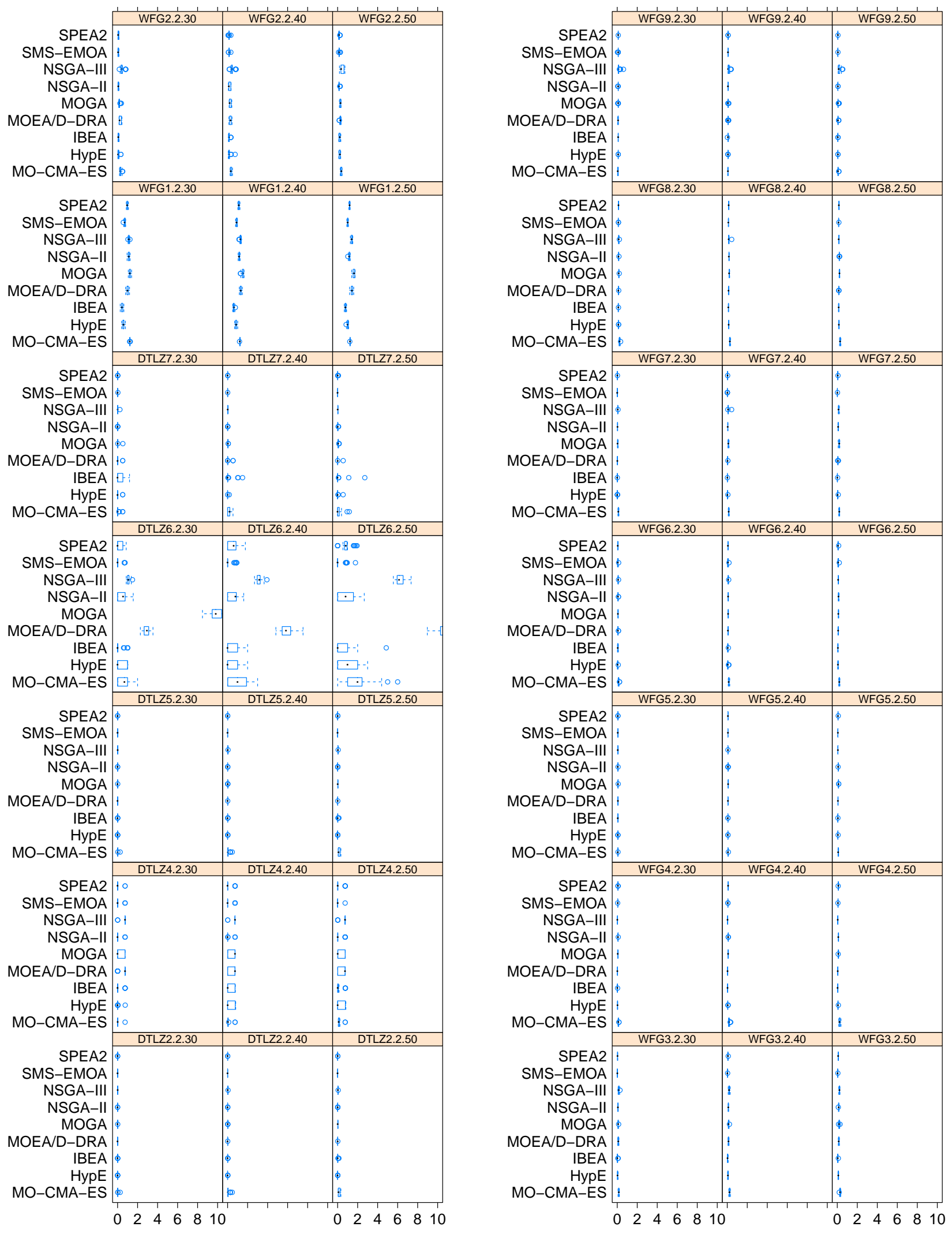

Figure 81: Boxplots of the $I_{I G D}$ for $M=2$ problems, $n_{\mathbf{v a r}} \in\{30,40,50\}$ and $\boldsymbol{F E}_{\max }=10000$. 

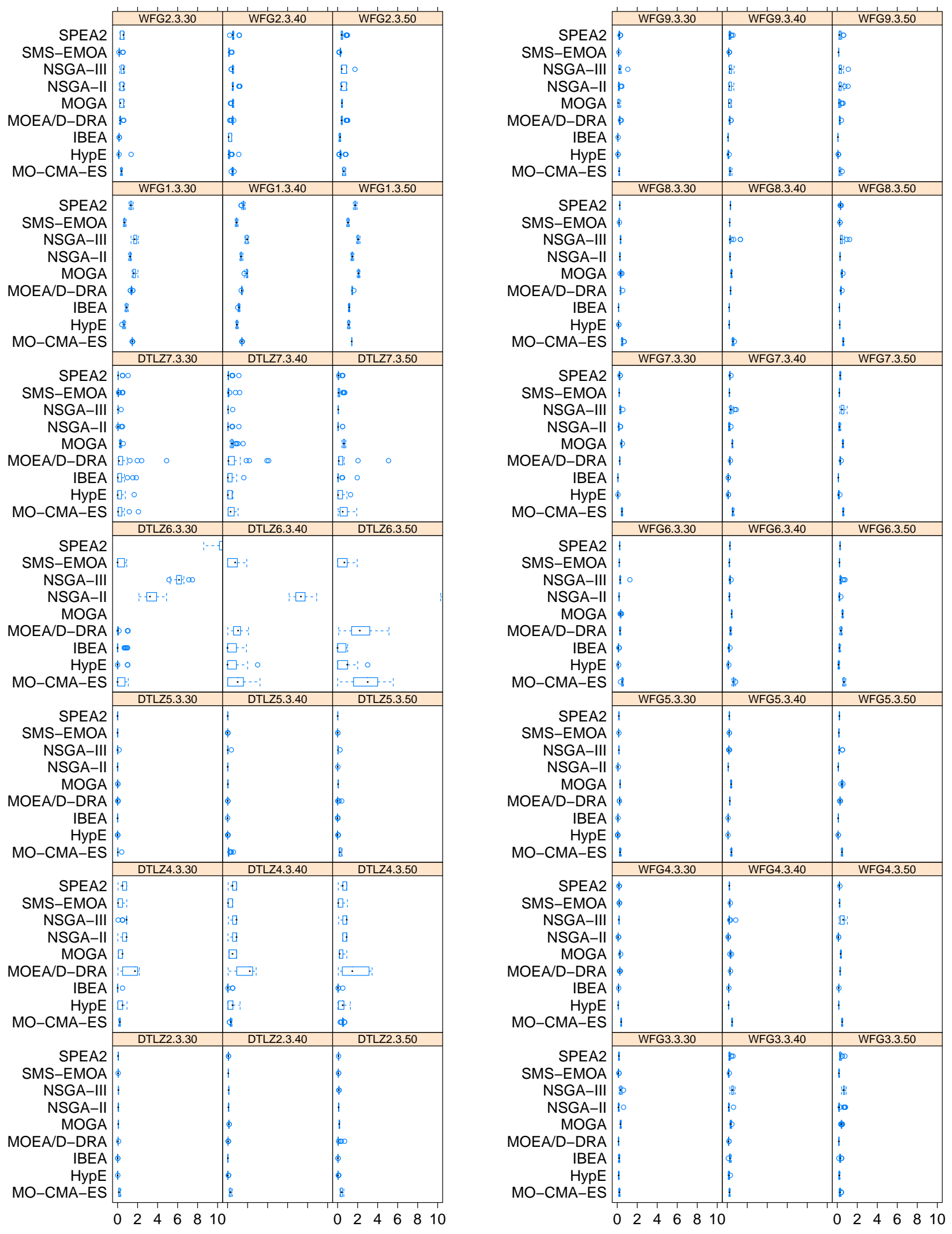

Figure 82: Boxplots of the $I_{I G D}$ for $M=3$ problems, $n_{\mathbf{v a r}} \in\{30,40,50\}$ and $\boldsymbol{F} E_{\max }=10000$. 

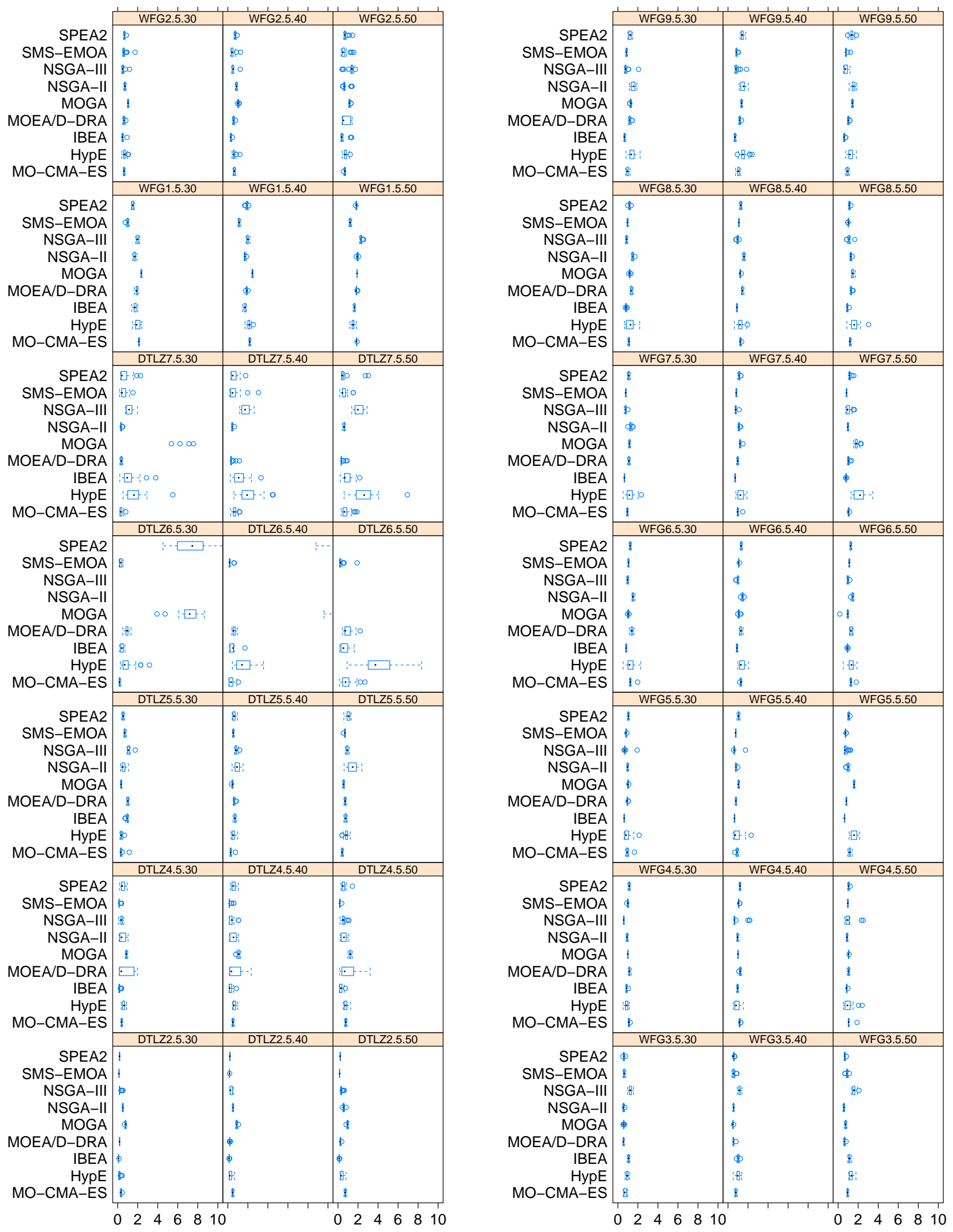

Figure 83: Boxplots of the $I_{I G D}$ for $M=5$ problems, $n_{\mathbf{v a r}} \in\{30,40,50\}$ and $\boldsymbol{F E}_{\max }=10000$. 

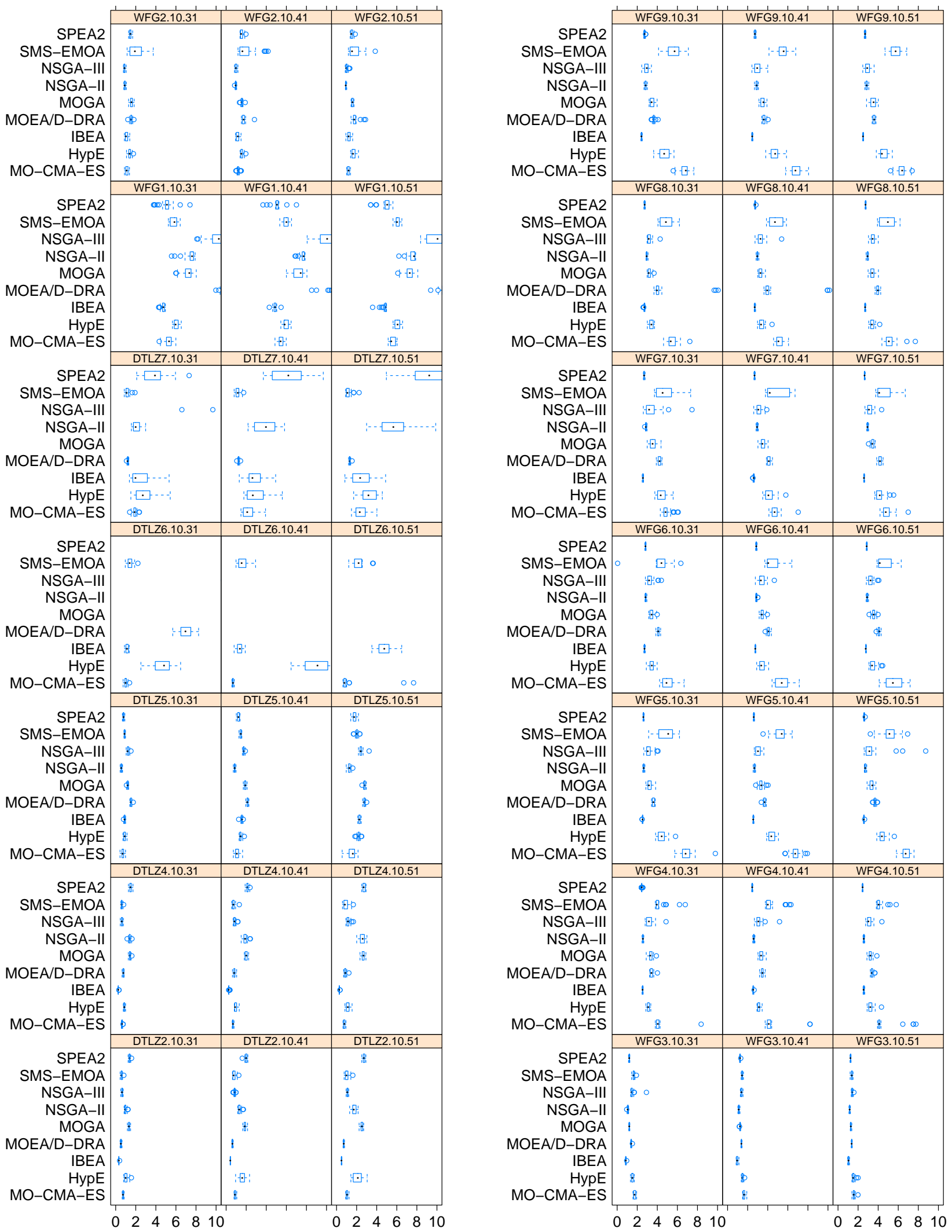

Figure 84: Boxplots of the $I_{I G D}$ for $M=10$ problems, $n_{\mathrm{var}} \in\{30,40,50\}$ and $\boldsymbol{F} \boldsymbol{E}_{\max }=10000$. 

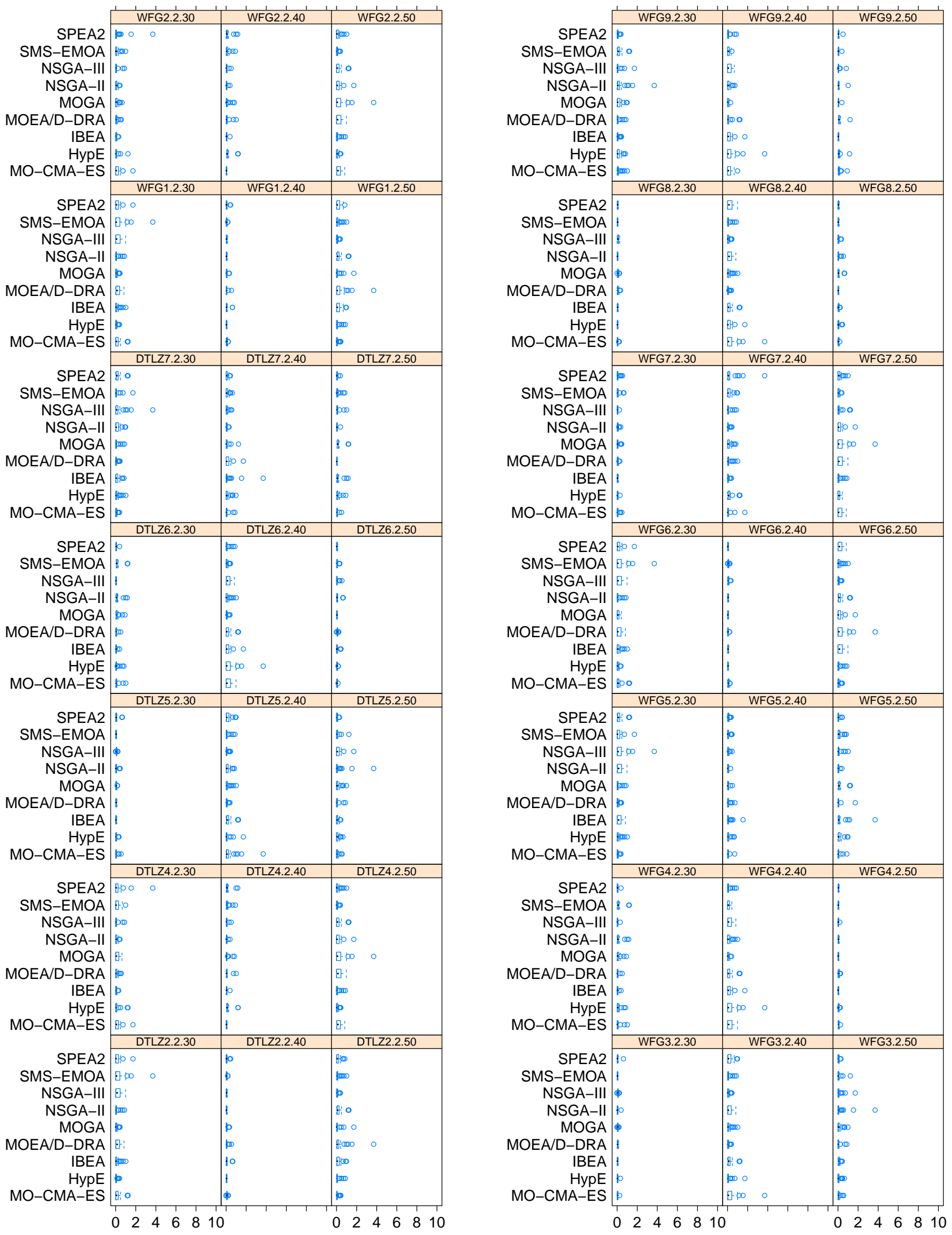

Figure 85: Boxplots of the $I_{I G D}$ for $M=2$ problems, $n_{\mathbf{v a r}} \in\{30,40,50\}$ and $\boldsymbol{F E}_{\mathbf{m a x}}=40000$. 

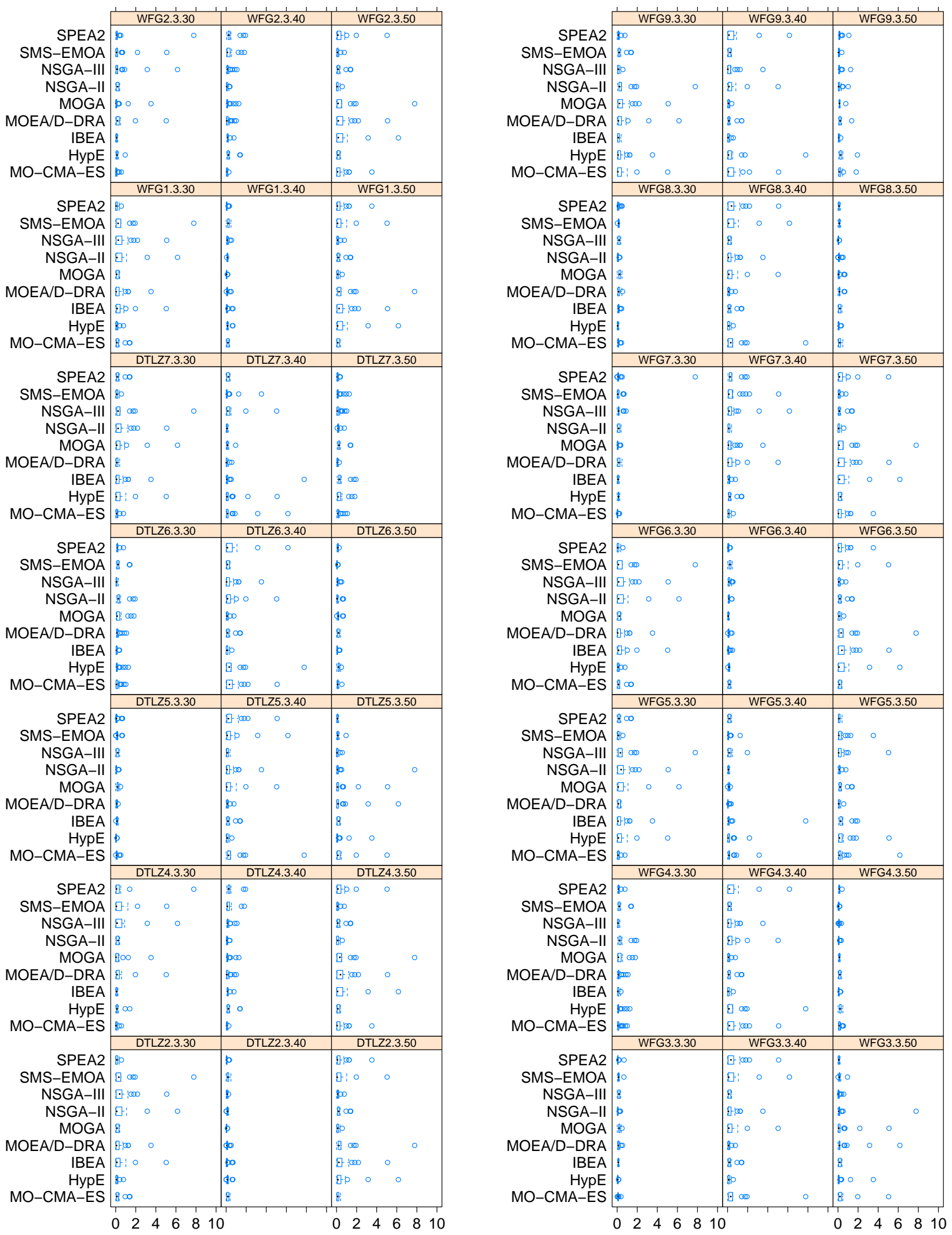

Figure 86: Boxplots of the $I_{I G D}$ for $M=3$ problems, $n_{\mathbf{v a r}} \in\{30,40,50\}$ and $\boldsymbol{F} E_{\max }=40000$. 

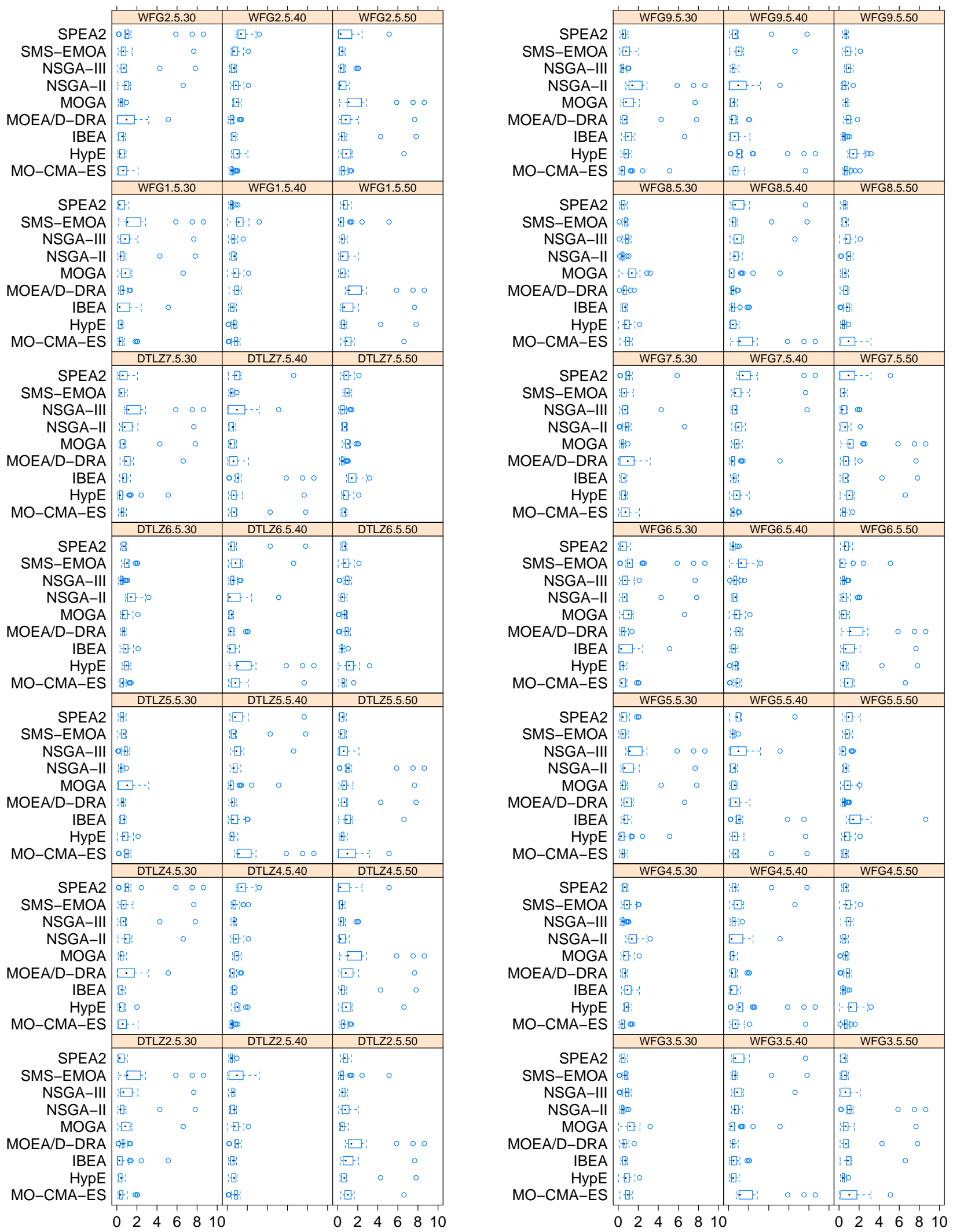

Figure 87: Boxplots of the $I_{I G D}$ for $M=5$ problems, $n_{\mathbf{v a r}} \in\{30,40,50\}$ and $\boldsymbol{F} E_{\max }=40000$. 

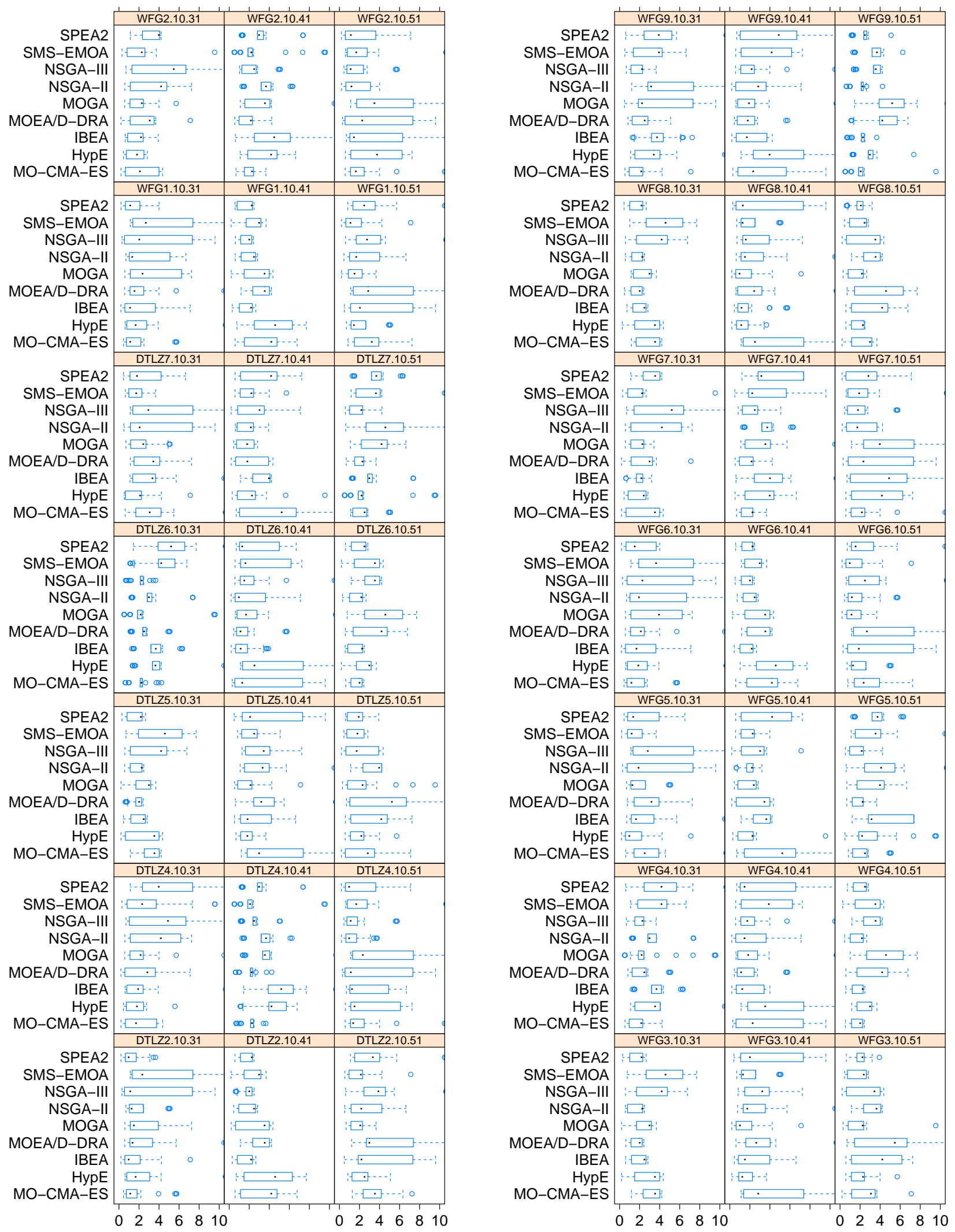

Figure 88: Boxplots of the $I_{I G D}$ for $M=10$ problems, $n_{\mathbf{v a r}} \in\{30,40,50\}$ and $\boldsymbol{F E}_{\mathbf{m a x}}=40000$. 


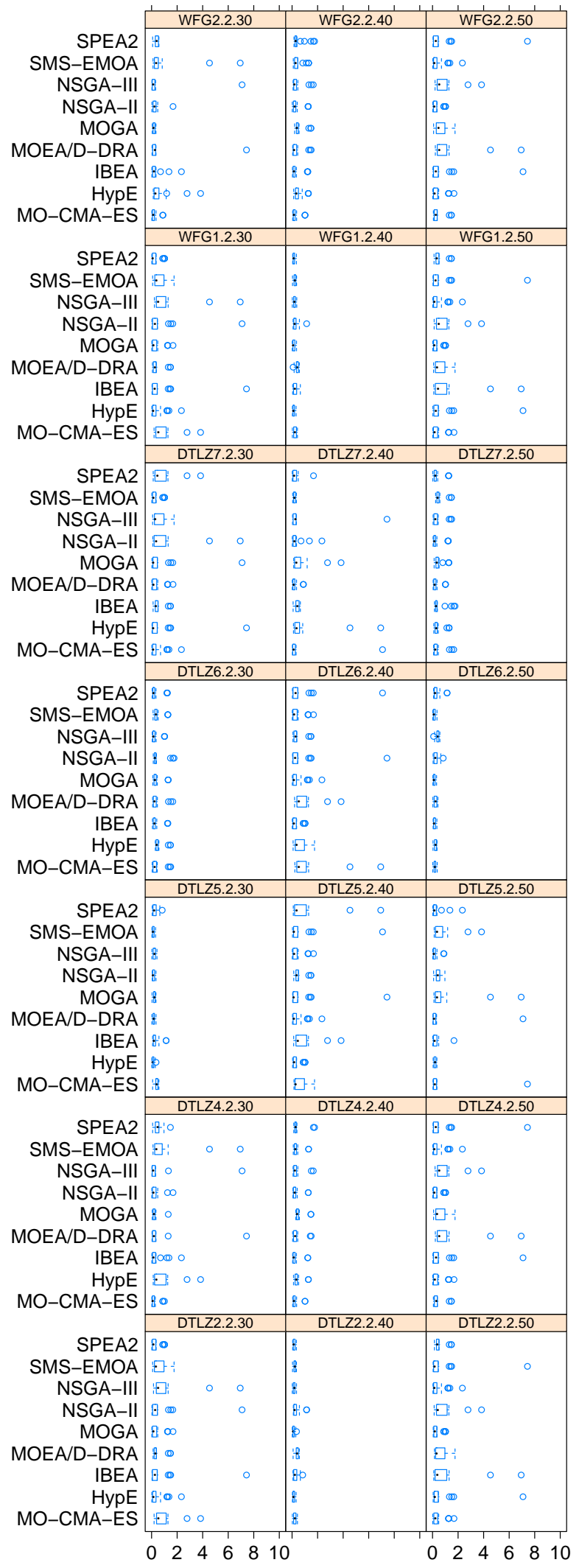

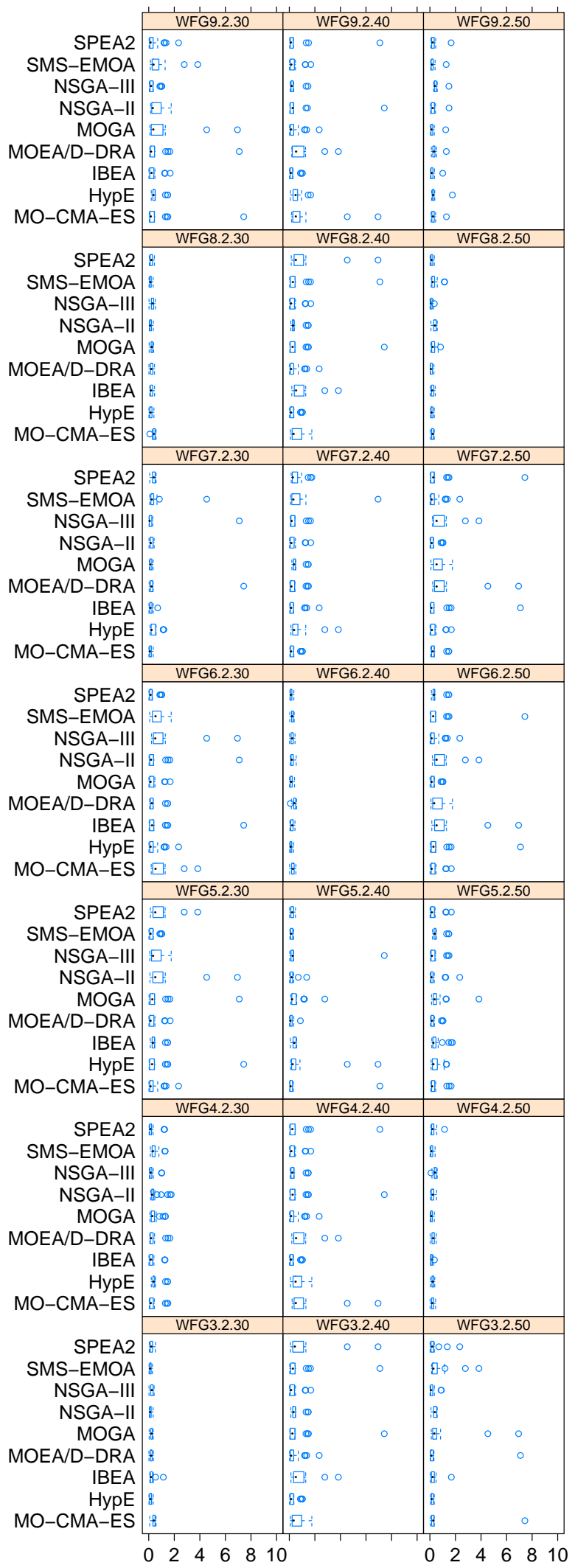

Figure 89: Boxplots of the $I_{I G D+}$ for $M=2$ problems, $n_{\mathbf{v a r}} \in\{30,40,50\}$ and $\boldsymbol{F E}_{\max }=2500$. 


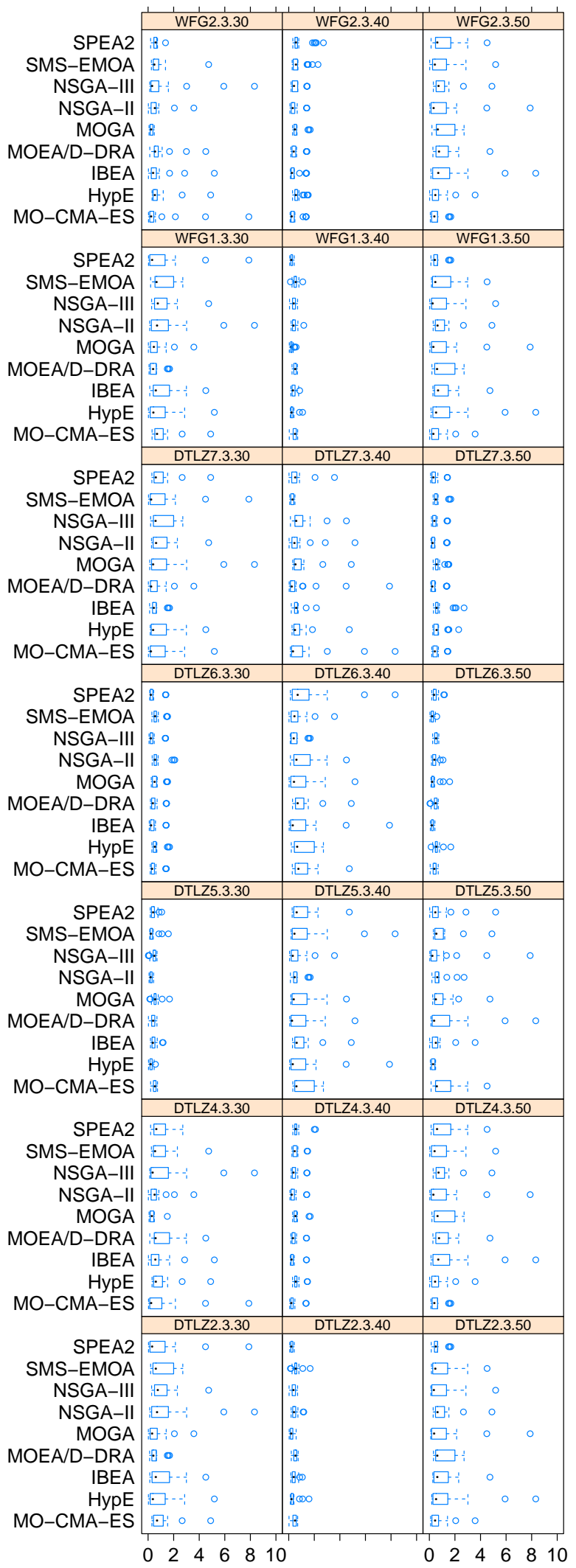

\begin{tabular}{|c|c|c|c|c|}
\hline \multirow{10}{*}{$\begin{array}{r}\text { SPEA2 } \\
\text { SMS-EMOA } \\
\text { NSGA-III } \\
\text { NSGA-II } \\
\text { MOGA } \\
\text { MOEA/D-DRA } \\
\text { IBEA } \\
\text { HypE } \\
\text { MO-CMA-ES }\end{array}$} & WFG9.3.30 & \multicolumn{2}{|c|}{ WFG9.3.40 } & WFG9.3.50 \\
\hline & $\begin{array}{lll}1 & 1 & 0 \\
y-1 & 0 & 0\end{array}$ & Hack & & 80 \\
\hline & F- 0 & 明。 & & 央 0 \\
\hline & $\square \div$ & $\theta^{-\frac{1}{1}} \circ$ & & \&is \\
\hline & $\theta+0$ & 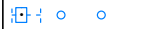 & & \#0 \\
\hline & $\because-i$ & 议: & & 采。 \\
\hline & 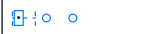 & :-1: 0 & $\circ$ & fio 0 \\
\hline & & $i \div \div$ & & 異。 \\
\hline & D- - & 沱+ & & $8 \circ$ \\
\hline & WFG8.3.30 & WFG8.3.4C & & WFG8.3.50 \\
\hline SPEA2 & 緒 & 呫-10 & & 渴 \\
\hline SMS-EMOA & : & $\because-\frac{1}{1} \quad 0$ & $\circ$ & fito \\
\hline NSGA-III & 糟 & 壮 $\div 00$ & & 焉 \\
\hline NSGA-II & 采 & & & 棌 \\
\hline MOGA & off & $\sqrt{\cdot}-\frac{1}{1}$ & & 貼 \\
\hline MOEA/D-DRA & igt & $: \square-\cdots$ & & $8 \infty \infty$ \\
\hline IBEA & 采 & 䋍: 。 & & 践 \\
\hline HypE & 䊗 & $D \div \frac{1}{1} \quad 0$ & $\circ$ & \&i \\
\hline MO-CMA-ES & is & $:-\frac{1}{1}$ & & offitio \\
\hline & WFG7.3.30 & WFG7.3.4 & & WFG7.3.50 \\
\hline SPEA2 & 星 & $\square-1$ & & $\square-\frac{1}{1}$ \\
\hline SMS-EMOA & tot & $\forall-1$ & & 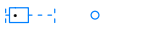 \\
\hline NSGA-III & 8000 & $\square$ & 。 & 四: 0 \\
\hline NSGA-II & & D00 ० & & $\theta \div \quad 0$ \\
\hline MOGA & & & & $\square \div$ \\
\hline MOEA/D-DRA & offlio o & $\square \quad \circ$ & & 细- $\div$ \\
\hline IBEA & 壮。 & 분: 0 & & $\square-10$ \\
\hline HypE & 跑 & 证: 0 & & 沺 $\div 0 \circ$ \\
\hline MO-CMA-ES & 80 & $\bigoplus: \quad \circ$ & $\circ$ & 沺。 \\
\hline & \begin{tabular}{|l} 
WFG6.3.30 \\
\end{tabular} & WFG6.3.4 & & WFG6.3.50 \\
\hline SPEA2 & $\square \div 00$ & 舟 & & 阐。 \\
\hline SMS-EMOA & : & 湈 & & $\sqrt{0}-40$ \\
\hline NSGA-III & 壮- & ift: & & $y=$ \\
\hline NSGA-II & $\because\left[\begin{array}{ll}1 & 0\end{array}\right.$ & 品 & & 䚹: 0 \\
\hline MOGA & 怔 †0。 & 舟 & & : \\
\hline MOEA/D-DRA & & 界 & & $4 \div$ \\
\hline IBEA & $: \square_{-1}^{-1} \circ$ & $f:$ & & 沺: \\
\hline HypЕ & 壮- - & $f_{i}$ & & $\therefore-10$ \\
\hline MO-CMA-ES & 河: 0 & ofi: & & 计†边 \\
\hline & WFG5.3.30 & WFG5.3.4 & & WFG5.3.50 \\
\hline SPEA2 & 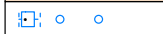 & 细。 & & 国。 \\
\hline SMS-EMOA & 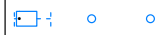 & & & 是。 \\
\hline NSGA-III & 弪 & oflioo $\circ \circ$ & & $\square$ \\
\hline NSGA-II & 㞹 & 酒○。 & & \\
\hline MOGA & $\because-1 \quad 0 \quad 0$ & 此：。 & & 惯如 \\
\hline MOEA/D-DRA & 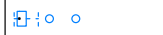 & $y 00$ & & \\
\hline IBEA & 团。 & & & : $\div 0$ \\
\hline HypE & 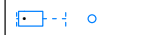 & 趴 0 & & 回-: \\
\hline MO-CMA-ES & 田--1 0 & $f \infty \infty \quad \circ \quad \circ$ & & $\square \quad \circ$ \\
\hline & \begin{tabular}{|l} 
WFG4.3.30 \\
\end{tabular} & WFG4.3.4 & & WFG4.3.50 \\
\hline SPEA2 & f100 & $\nabla-10$ & & ËO \\
\hline SMS-EMOA & iflo & 泟:0。 & & s \\
\hline NSGA-III & $\exists \infty$ & & & 悉 \\
\hline NSGA-II & 1000 & $\square^{-\frac{1}{1}} \circ$ & & 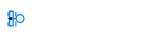 \\
\hline MOGA & 000 & : & & fit \\
\hline MOEA/D-DRA & 甲。 & 四: 。 & & $\phi \mathbb{B}_{1}^{\prime}$ \\
\hline IBEA & 虾。 & $D+0$ & $\circ$ & p \\
\hline HypE & 90 & $: \square \div$ & & 沘 \\
\hline MO-CMA-ES & 㥜。 & 计-1 0 & & 垏 \\
\hline & WFG3.3.30 & WFG3.3.4 & & WFG3.3.50 \\
\hline SPEA2 & 船 & 田! 0 & & 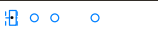 \\
\hline SMS-EMOA & fio & {$\left[\begin{array}{ll}-1 & 0\end{array}\right.$} & & 酙 0 \\
\hline NSGA-III & it & 汶 $\div 00$ & & 思 00 \\
\hline NSGA-II & $\$$ & & & \\
\hline MOGA & 活 & $\theta-\frac{1}{1} 0$ & & 畞 \\
\hline MOEA/D-DRA & 海 & : & & $8000 \circ \quad \circ$ \\
\hline IBEA & $\$ 0$ & 怩: 00 & & 勘 \\
\hline HypE & p & $10 \div 0$ & $\circ$ & \\
\hline MO-CMA-ES & 早 & $\sqrt{-1}$ & & it: $0 \circ 0$ \\
\hline & 02 & & & \\
\hline
\end{tabular}

Figure 90: Boxplots of the $I_{I G D+}$ for $M=3$ problems, $n_{\mathbf{v a r}} \in\{30,40,50\}$ and $\boldsymbol{F} E_{\max }=2500$. 

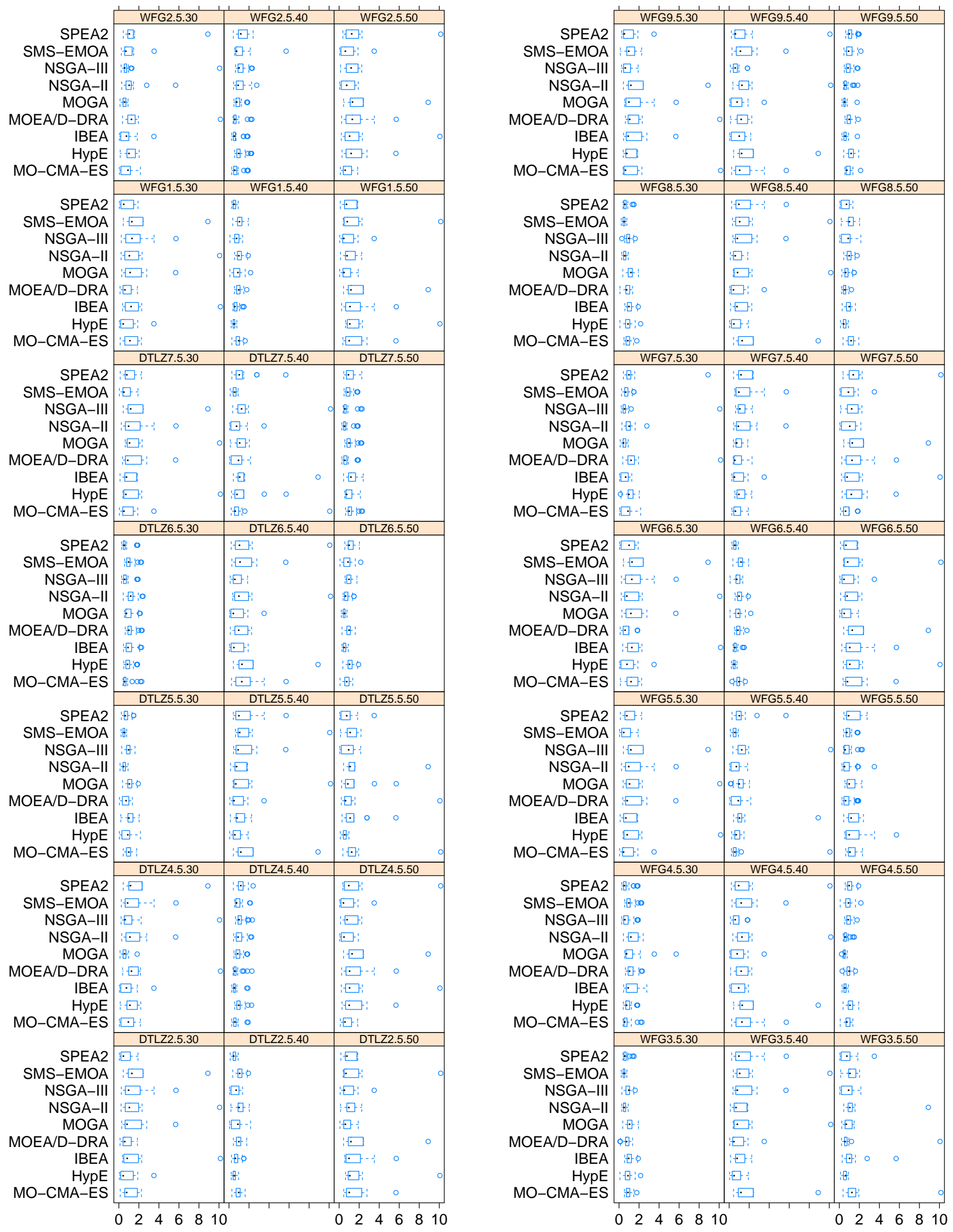

Figure 91: Boxplots of the $I_{I G D+}$ for $M=5$ problems, $n_{\mathbf{v a r}} \in\{30,40,50\}$ and $\boldsymbol{F E}_{\max }=2500$. 

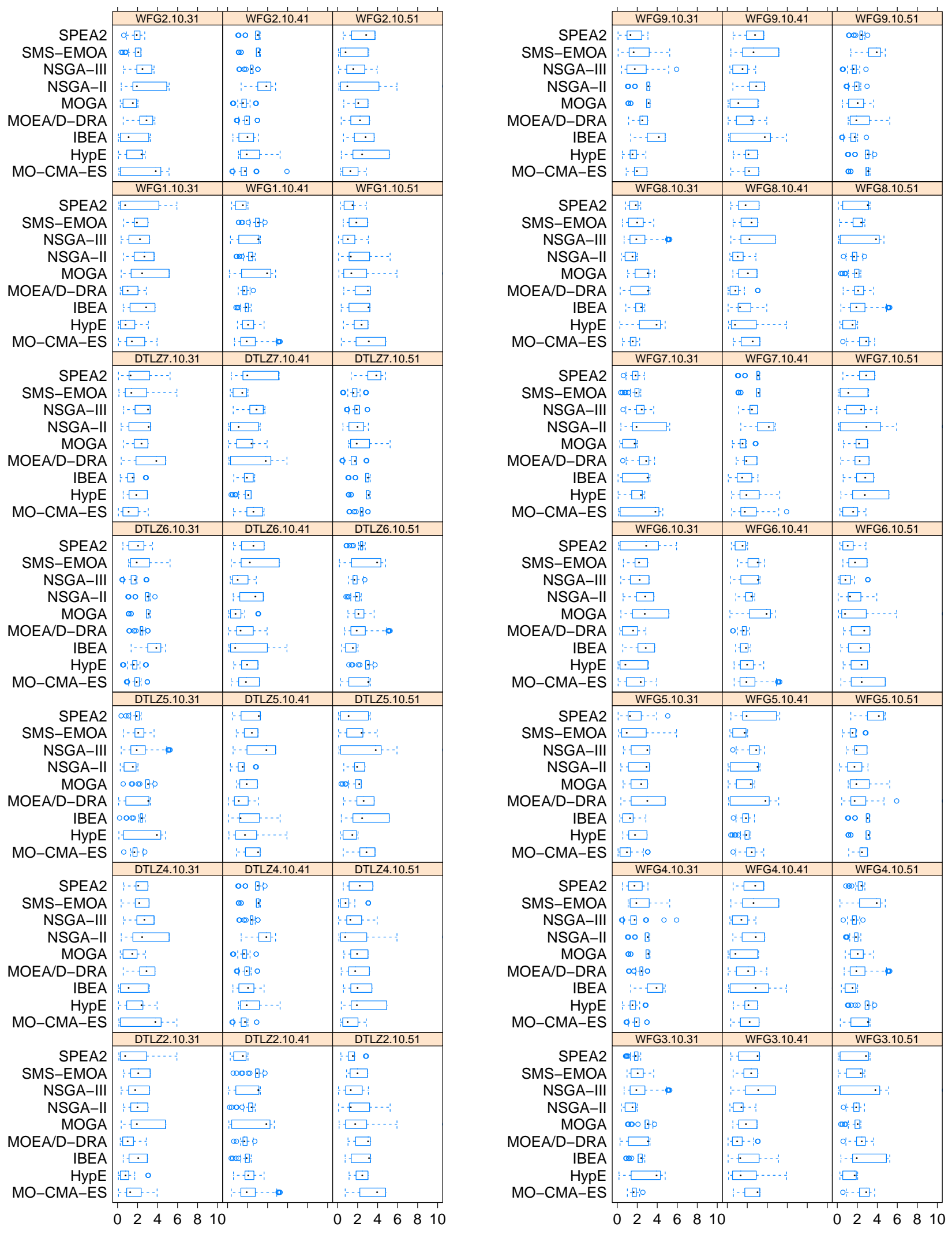

Figure 92: Boxplots of the $I_{I G D+}$ for $M=10$ problems, $n_{\mathbf{v a r}} \in\{30,40,50\}$ and $\boldsymbol{F} E_{\max }=2500$. 

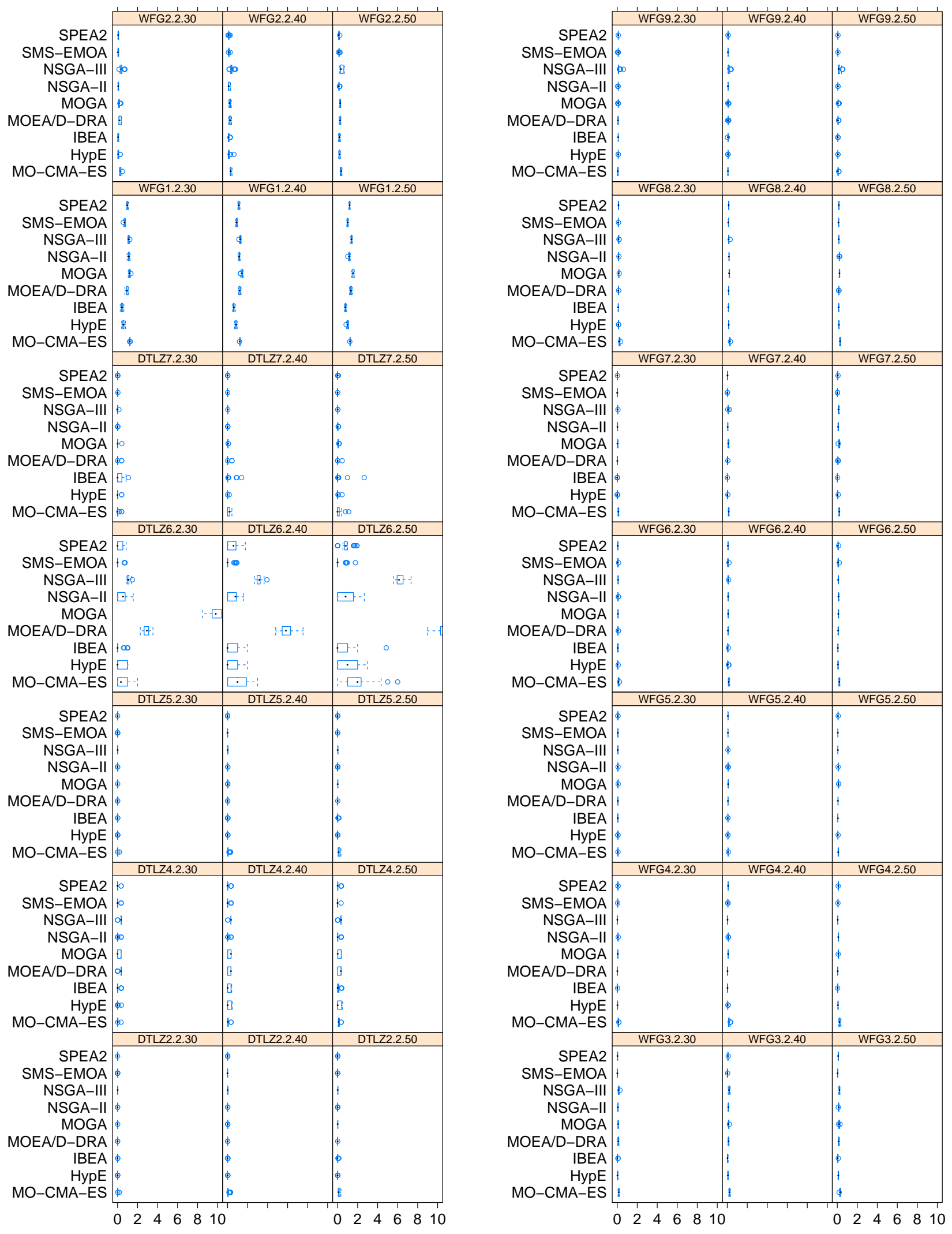

Figure 93: Boxplots of the $I_{I G D+}$ for $M=2$ problems, $n_{\mathbf{v a r}} \in\{30,40,50\}$ and $\boldsymbol{F E}_{\max }=10000$. 

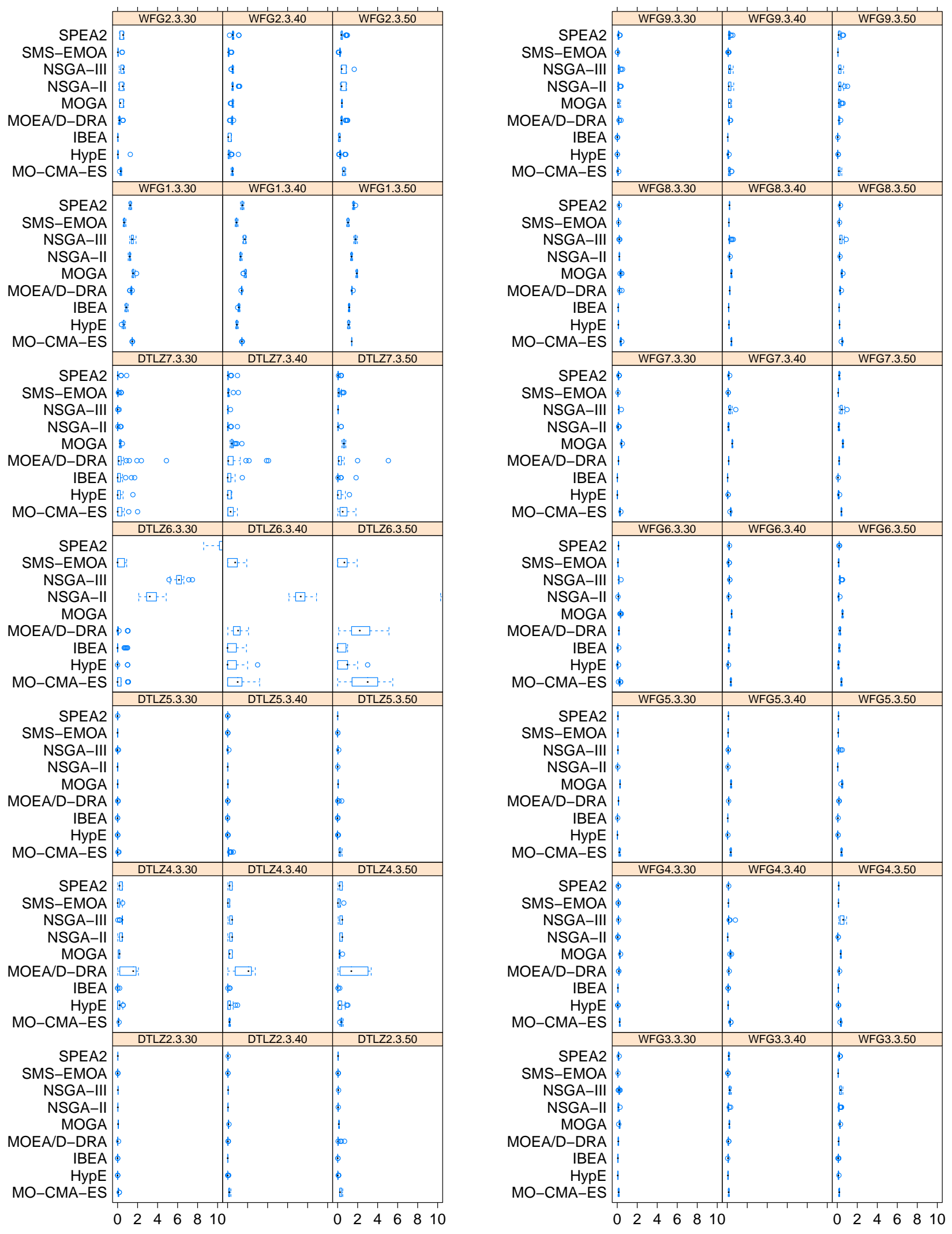

Figure 94: Boxplots of the $I_{I G D+}$ for $M=3$ problems, $n_{\mathbf{v a r}} \in\{30,40,50\}$ and $\boldsymbol{F} E_{\max }=10000$. 

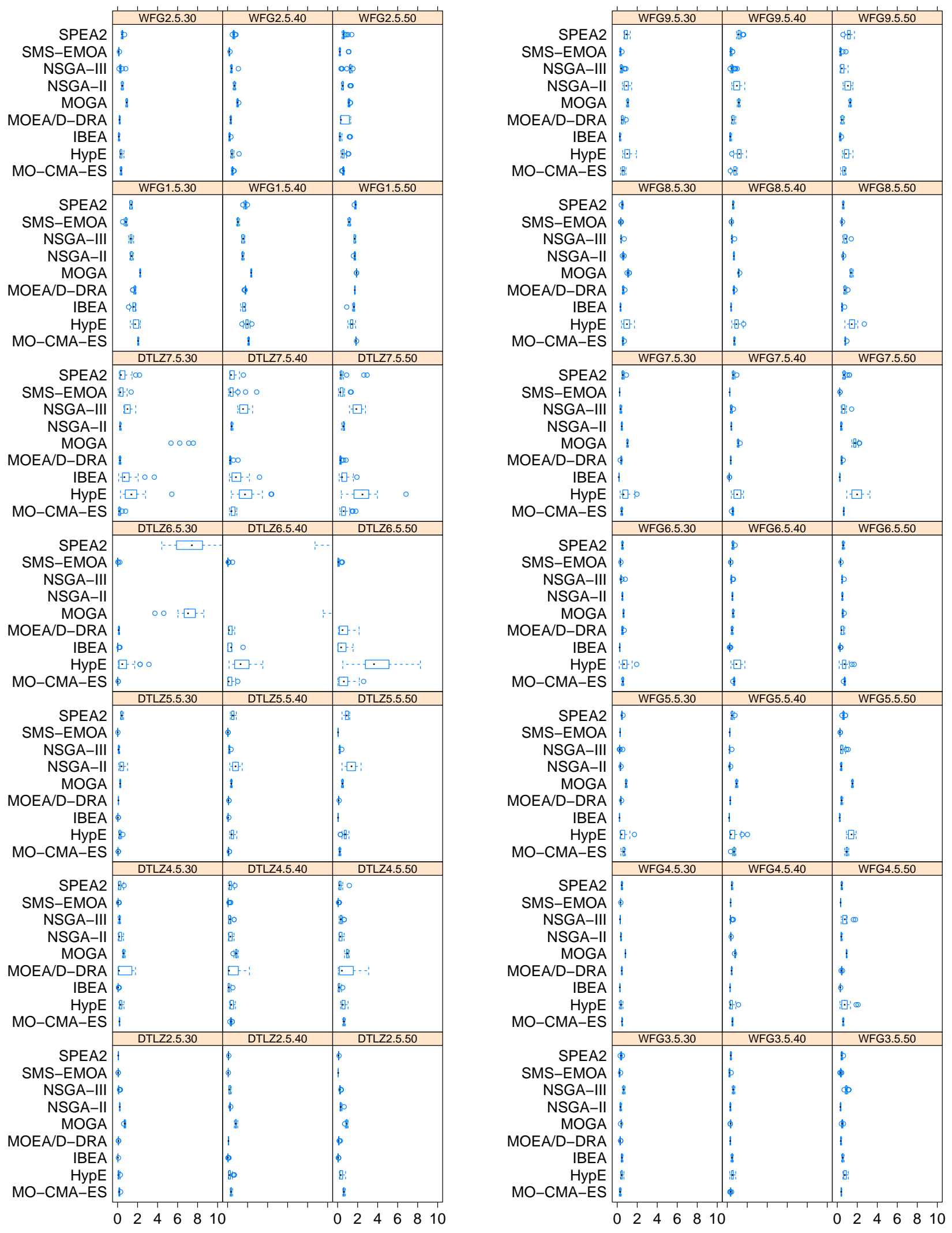

Figure 95: Boxplots of the $I_{I G D+}$ for $M=5$ problems, $n_{\mathbf{v a r}} \in\{30,40,50\}$ and $\boldsymbol{F} E_{\max }=10000$. 

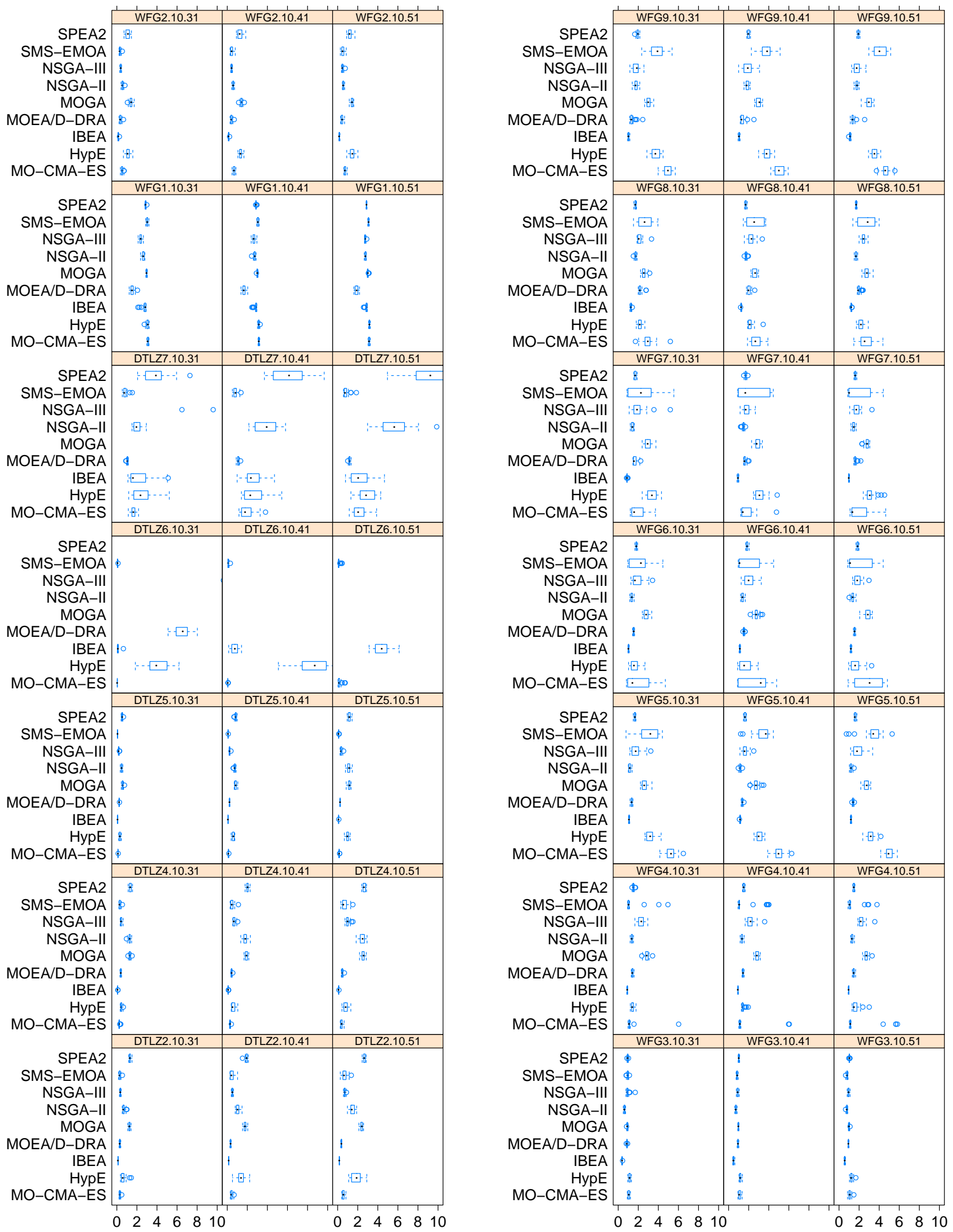

Figure 96: Boxplots of the $I_{I G D+}$ for $M=10$ problems, $n_{\mathbf{v a r}} \in\{30,40,50\}$ and $\boldsymbol{F E}_{\mathbf{m a x}}=10000$. 

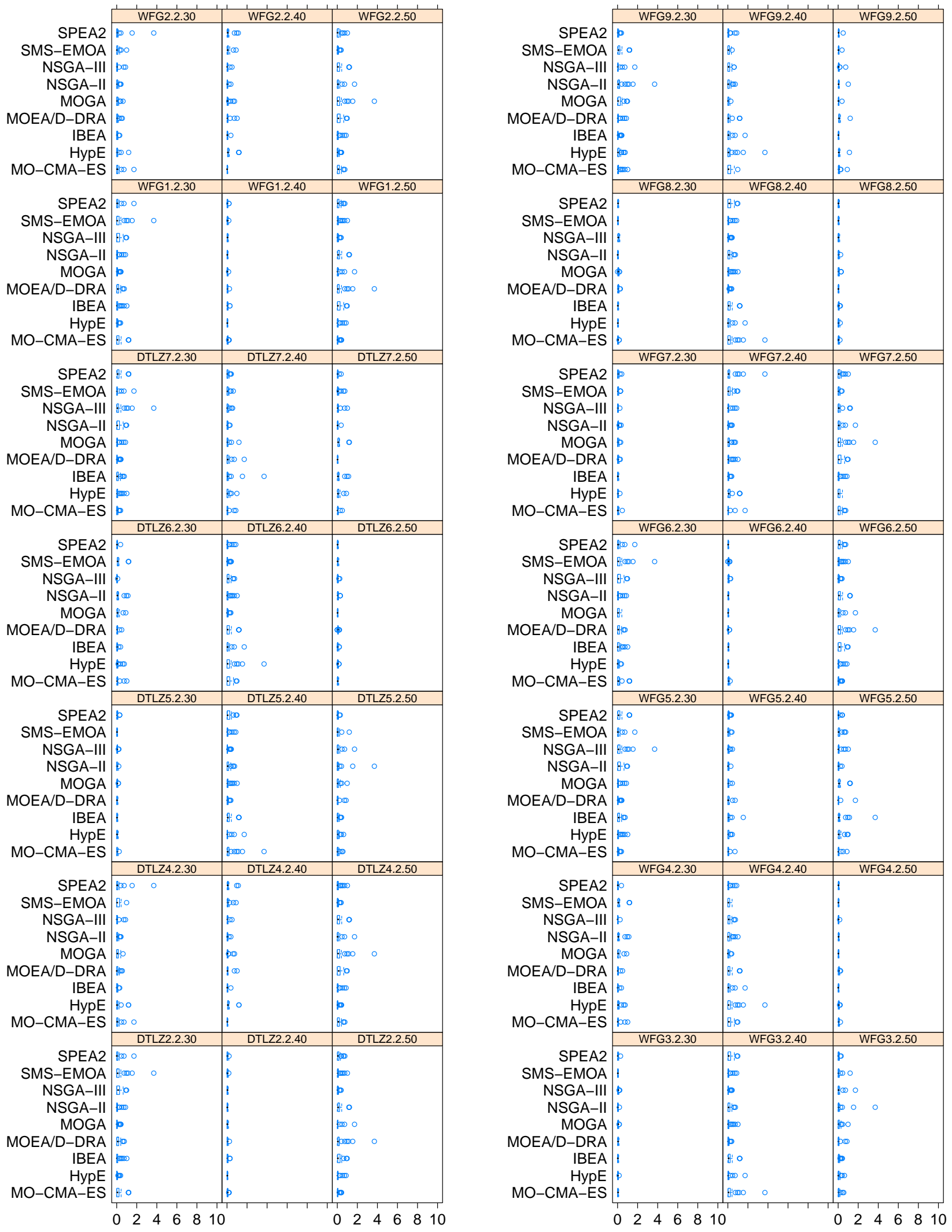

Figure 97: Boxplots of the $I_{I G D+}$ for $M=2$ problems, $n_{\mathrm{var}} \in\{30,40,50\}$ and $\boldsymbol{F} \boldsymbol{E}_{\max }=40000$. 

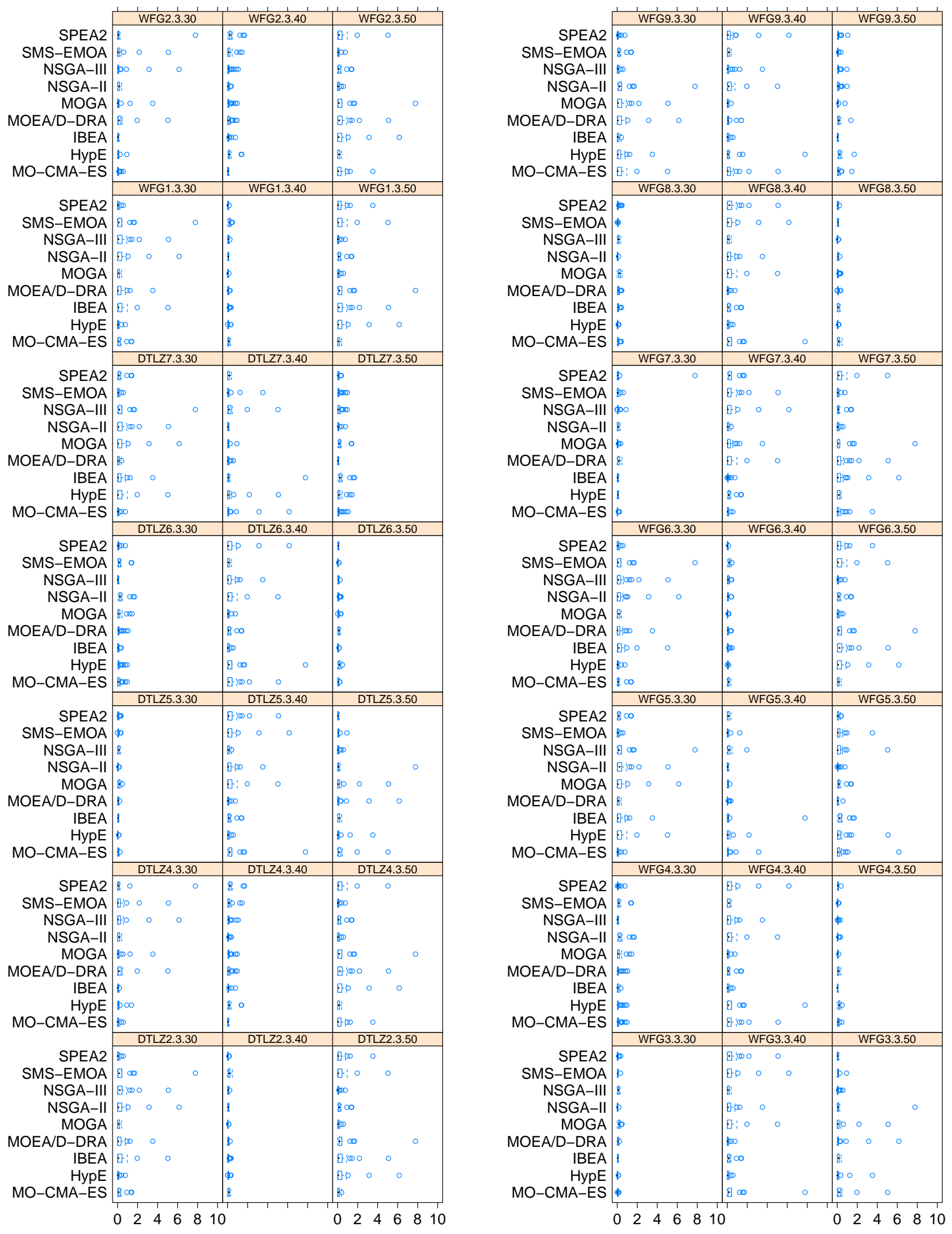

Figure 98: Boxplots of the $I_{I G D+}$ for $M=3$ problems, $n_{\mathbf{v a r}} \in\{30,40,50\}$ and $\boldsymbol{F} \boldsymbol{E}_{\max }=40000$. 

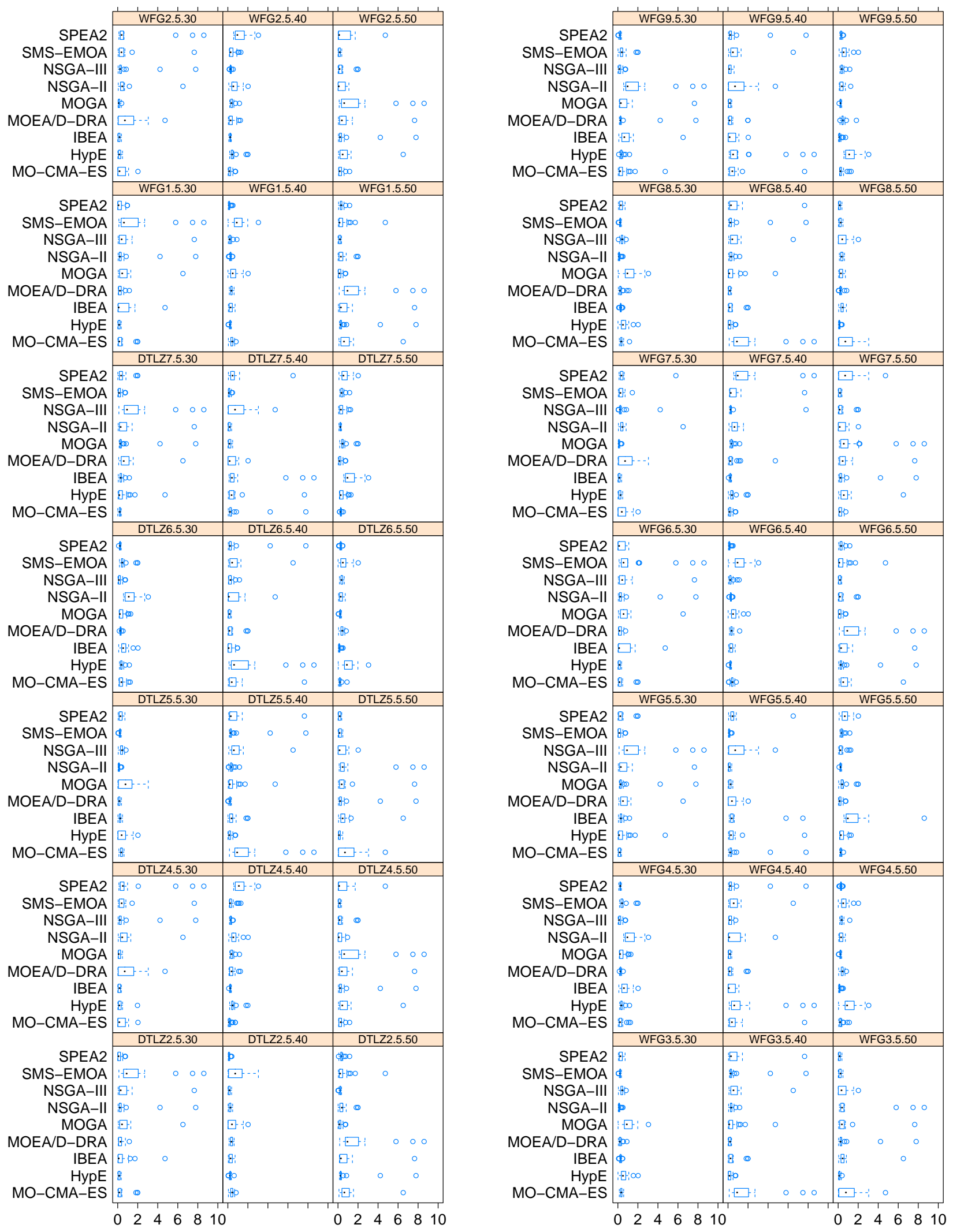

Figure 99: Boxplots of the $I_{I G D+}$ for $M=5$ problems, $n_{\mathbf{v a r}} \in\{30,40,50\}$ and $\boldsymbol{F E}_{\max }=40000$. 

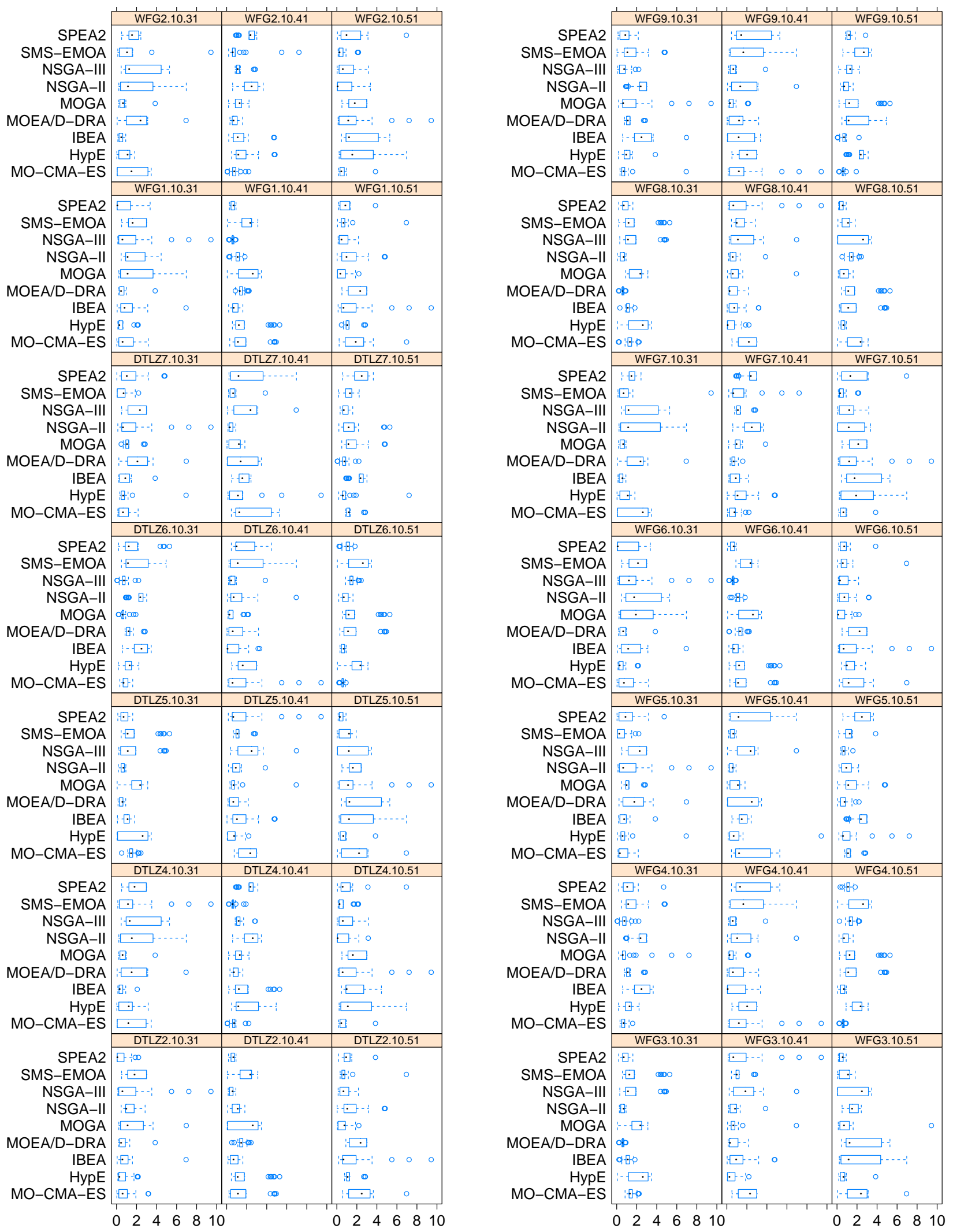

Figure 100: Boxplots of the $I_{I G D+}$ for $M=10$ problems, $n_{\mathrm{var}} \in\{30,40,50\}$ and $\boldsymbol{F} \boldsymbol{E}_{\max }=40000$. 